\title{
Functional analysis of ryanodine receptor 2 mutations in induced pluripotent stem cell- derived cardiomyocytes from CPVT patients
}

\author{
Doctoral Thesis
}

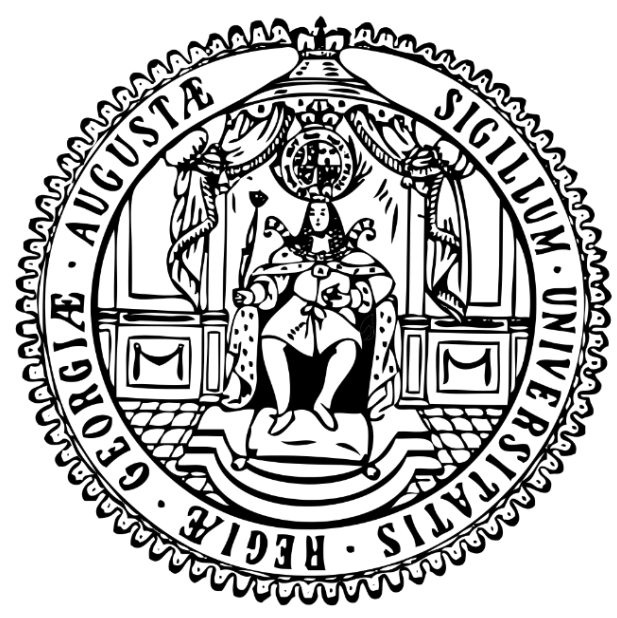

In partial fulfillment of the requirements for the degree

"Doctor rerum naturalium (Dr. rer. nat.)"

in the Molecular Medicine Study Program

at the Georg-August University Göttingen

submitted by

Wener Li

born in Hubei, P. R. China

Göttingen 2017 


\section{Members of the Thesis Committee:}

First member of the thesis committee/Supervisor:

Prof. Dr. rer. nat. Kaomei Guan

Institute of Pharmacology and Toxicology

Technische Universität Dresden

Second member of the thesis committee:

Prof. Dr. med. Swen Hülsmann

Department of Neurophysiology

Universitätsmedizin Göttingen

Third member of the thesis committee:

Prof. Dr. rer. nat. Viacheslav Nikolaev

Institute of Experimental Cardiovascular Research

University Medical Center Hamburg-Eppendorf

Date of Disputation: $1^{\text {st }}$ March 2017 


\section{AFFIDAVIT}

Here I declare that my doctoral thesis entitled "Functional analysis of ryanodine receptor 2 mutations in induced pluripotent stem cell-derived cardiomyocytes from CPVT patients" has been written independently with no other sources and aids than quoted.

Wener Li

Göttingen, January 2017 


\section{List of Publications}

\section{Peer-reviewed publications:}

Li, W.E., Chen, W., Ma, Y.F., Tuo, Q.R., Luo, X.J., Zhang, T., Sai, W.B., Liu, J., Shen, J., Liu, Z.G., et al. (2012). Methods to measure and analyze ciliary beat activity: $\mathrm{Ca}^{2+}$ influx-mediated cilia mechanosensitivity. Pflugers Arch 464, 671-680.

Zhang, T., Luo, X.J., Sai, W.B., Yu, M.F., Li, W.E., Ma, Y.F., Chen, W., Zhai, K., Qin, G., Guo, D., et al. (2014). Non-selective cation channels mediate chloroquine-induced relaxation in precontracted mouse airway smooth muscle. PloS one 9, e101578.

\section{Conference abstracts:}

Cyganek, L., Li, W., Henze, S., Chen, S., Bezzina, C. R., Wilde, A. A., Guan, K. Functional analysis of distinct ryanodine receptor type 2 mutations in induced pluripotent stem cell-derived cardiomyocytes from CPVT patients. EUROPEAN JOURNAL OF HEART FAILURE, 2015, 17:236; Heart Failure Congress of the European-Society-of-Cardiology (ESC), MAY 23-26, 2015, Seville, Spain.

Cyganek, L., Sekeres, K., Henze, S., Li, W., Guan, K. Custom-tailored cardiomyocytes: a directed in vitro differentiation of human pluripotent stem cells into defined cardiomyocyte subtypes. EUROPEAN JOURNAL OF HEART FAILURE, 2015, 17:370; Heart Failure Congress of the European-Society-of-Cardiology (ESC), MAY 23-26, 2015, Seville, Spain.

Stauske, M., Wagner, S., Li, W., Maier, L., Hasenfuss, G., Guan, K. Modelling Brugada syndrome using patient-specific induced pluripotent stem cells. EUROPEAN HEART JOURNAL, 2014, 35:380; Congress of the European-Society-of-Cardiology (ESC), AUG 30SEP 03, 2014, Barcelona, Spain

\section{Poster:}

Li W., Cyganek L., Chen S., Bezzina C.R., Wilde A.A.M., Guan K. Functional analysis of distinct ryanodine receptor type 2 mutations in induced pluripotent stem cell-derived 
cardiomyocytes from CPVT patients. $5^{\text {th }}$ International Congress on Stem Cells and Tissue Formation. JUL 8-11, 2014, Dresden, Germany. 


\section{Contents}

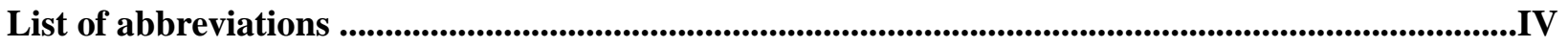

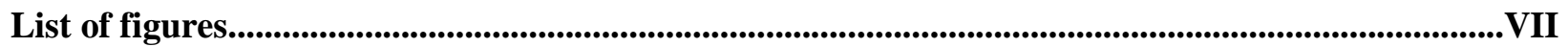

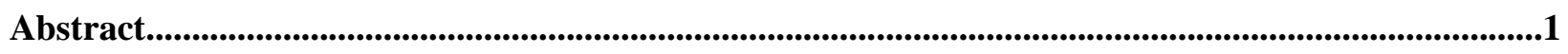

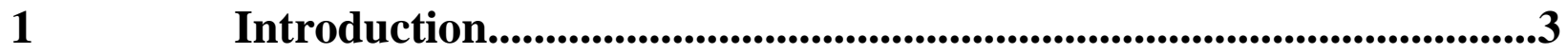

1.1 Catecholaminergic polymorphic ventricular tachycardia ............................................ 3

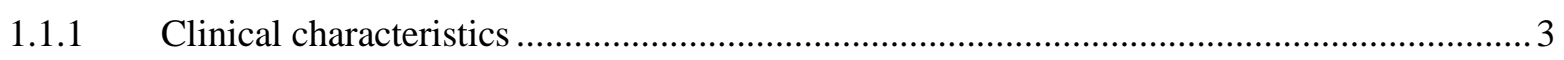

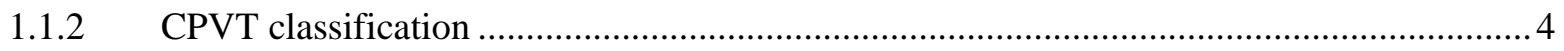

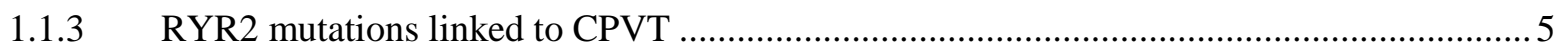

1.1.4 Pathophysiological mechanisms of CPVT triggered by RYR2 mutations ........................ 9

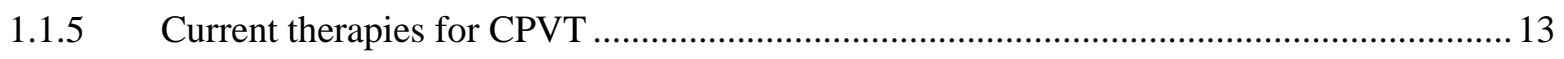

1.2 Application of human induced pluripotent stem cells in cardiac research ...................... 14

1.2.1 Human induced pluripotent stem cells ....................................................................... 15

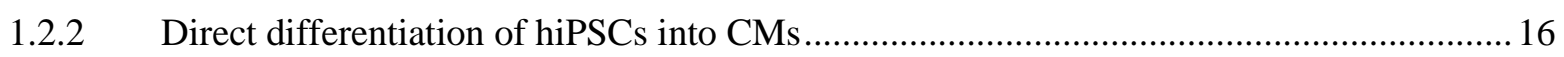

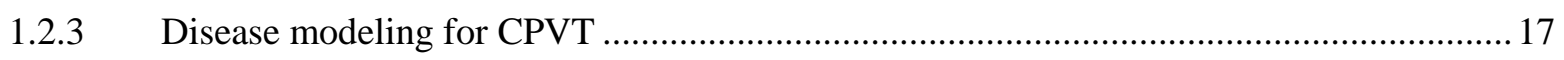

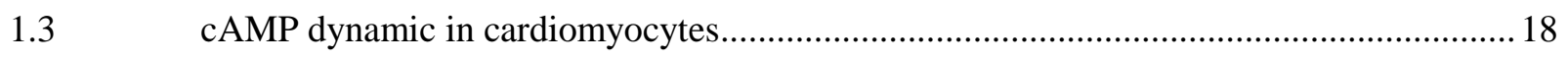

1.3.1 Altered cAMP dynamic in cardiovascular disease ......................................................... 19

1.3.2 Fluorescence resonance energy transfer............................................................... 21

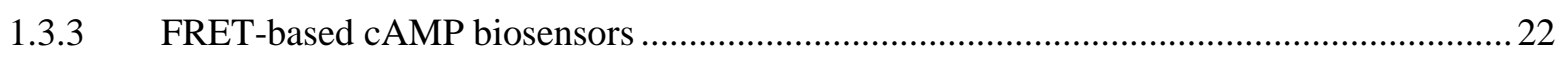

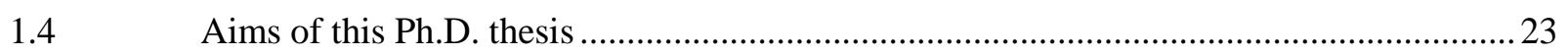

$2 \quad$ Materials and methods...................................................................25

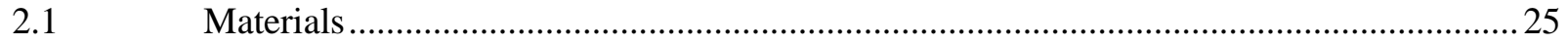

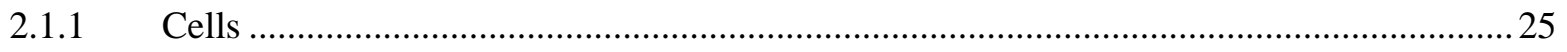

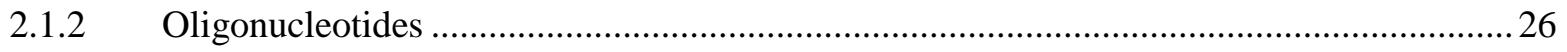

2.1.3 Sendai virus system used for cell reprogramming ........................................................ 27

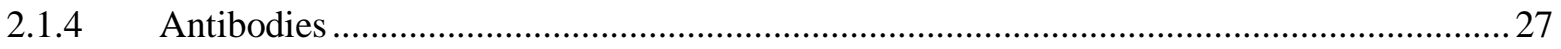

2.1.5 Media, solutions, and chemicals used for cell culture..................................................29

2.1.6 Solutions, buffers, and chemicals for molecular biology, FRET and electrophysiological

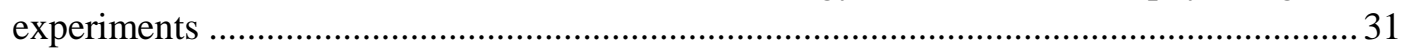

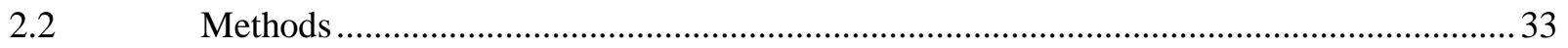

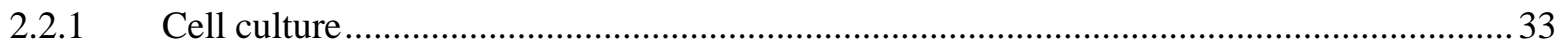

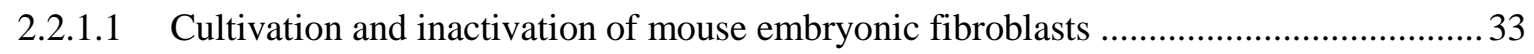

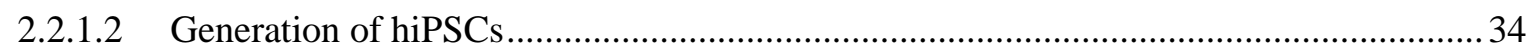




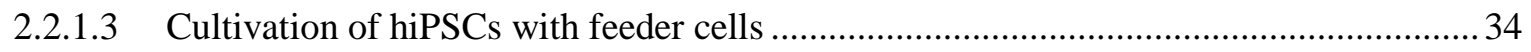

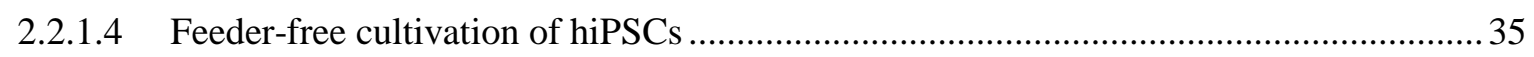

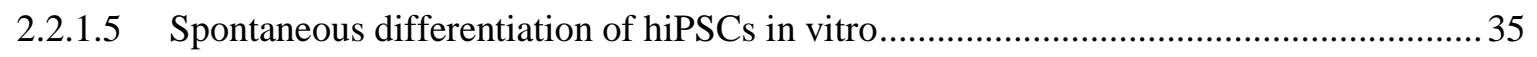

2.2.1.6 Directed differentiation of hiPSCs into CMs and CM digestion and culture..................35

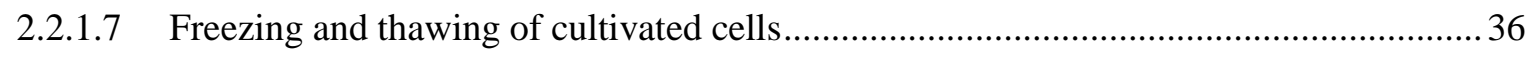

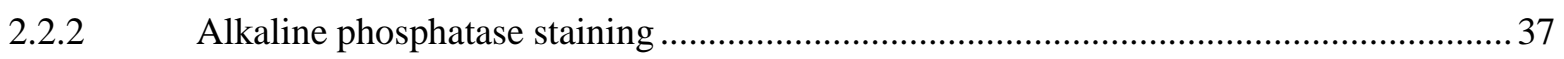

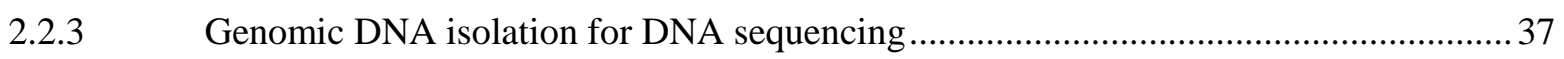

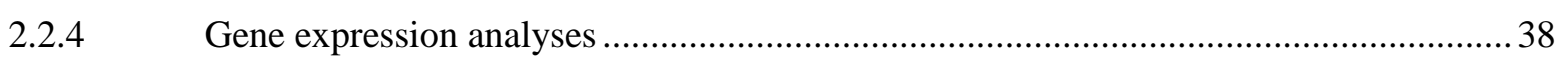

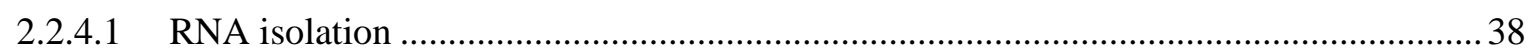

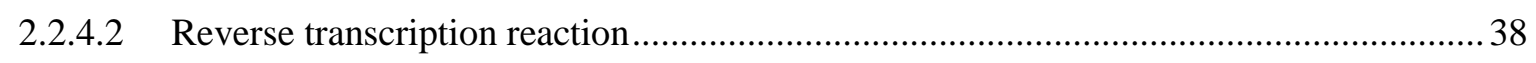

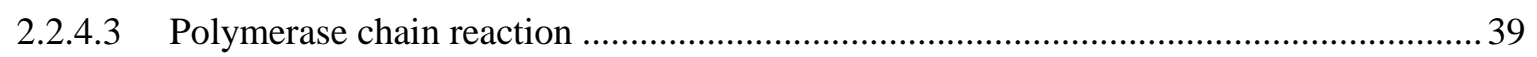

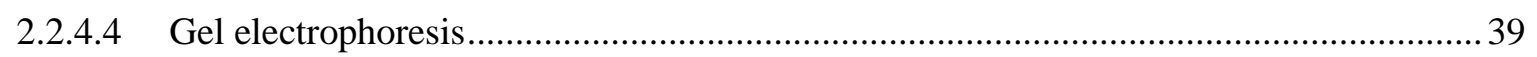

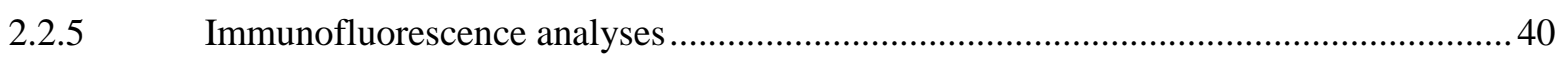

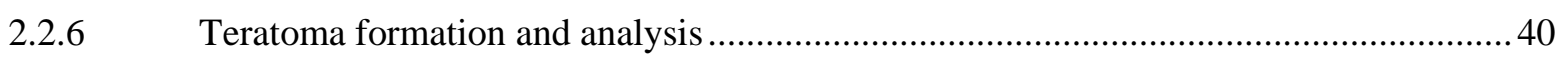

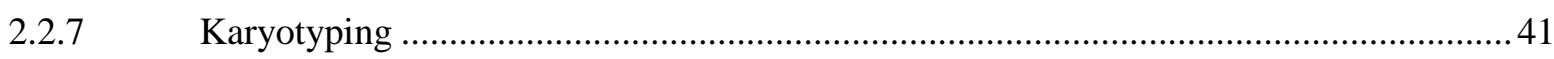

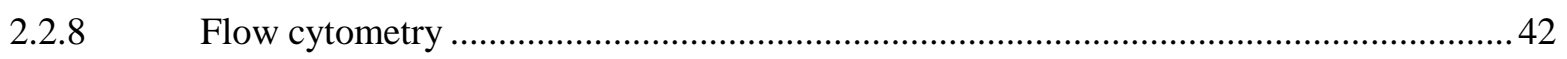

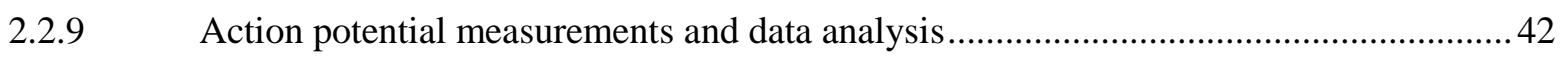

2.2.10 Microelectrode array experiments and data analysis ............................................... 43

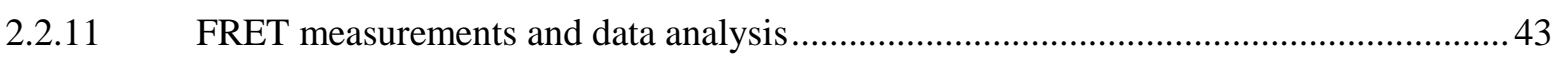

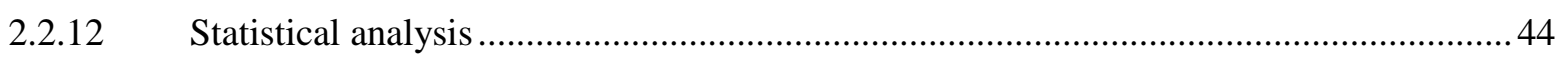

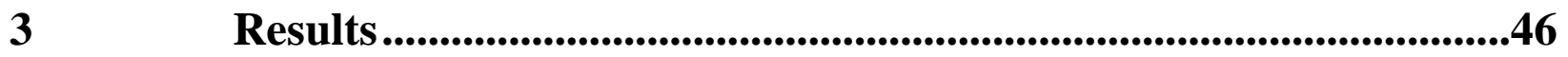

3.1 Generation of hiPSCs and proof of pluripotency …................................................ 46

3.1.1 Expression of pluripotency-related markers …................................................................ 46

3.1.2 Differentiation potential in vitro and in vivo ........................................................ 48

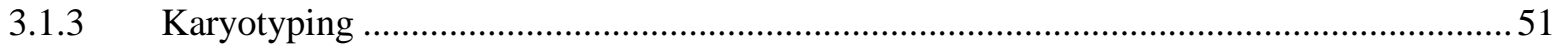

3.1.4 Verification of the RYR2 point mutation in CPVTa- and CPVTd-iPSCs ........................52

3.2 Generation and electrophysiological characterization of hiPSC-CMs ............................53

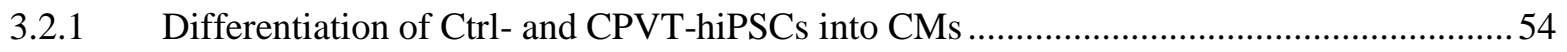

3.2.2 Action potential morphology of Ctrl-CMs and CPVT-CMs...........................................55

3.2.3 The characteristics of CPVT-CMs in basal condition..................................................... 60

3.2.4 CPVT-CMs after ISO stimulation recapitulated the disease phenotype of CPVT..............65

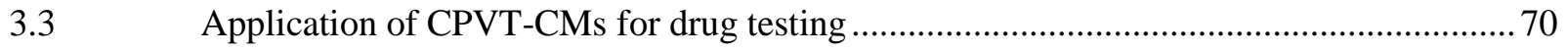

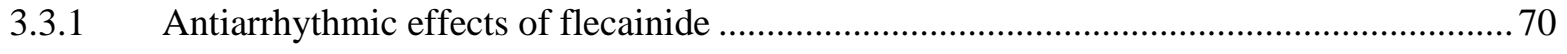

3.3.2 Antiarrhythmic effects of dantrolene .......................................................................... 73 


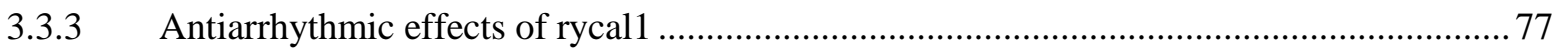

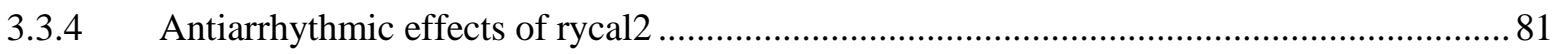

3.4 cAMP dynamics in Epac1-camps- and Epac1-camps-JNC-transfected Ctrl- and CPVT-

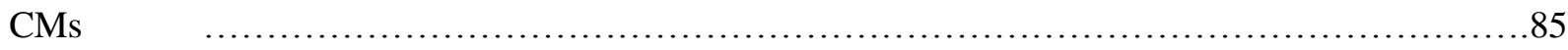

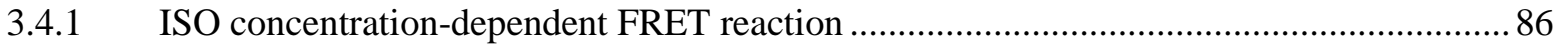

3.4.2 cAMP degradation by PDE2 hydrolytic activity in iPSC-CMs after $\beta$-adrenergic stimulation

3.4.3 cAMP degradation by PDE3 hydrolytic activity in iPSC-CMs after $\beta$-adrenergic stimulation

3.4.4 cAMP degradation by PDE4 hydrolytic activity in iPSC-CMs after $\beta$-adrenergic

4.1 Generation and characterization of hiPSCs and iPSC-CMs differentiation .96

4.2 CPVT-CMs can recapitulate electrophysiological phenotypes and imply distinguish

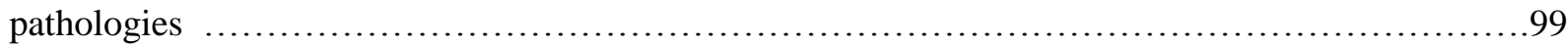

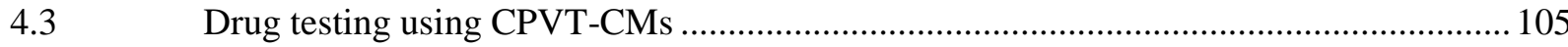

4.3.1 Two clinical drugs (flecainide and dantrolene) show their antiarrhythmic effects on CPVT(a, b, c)-CMs but not on CPVTd-CMs

4.3.2 Two experimental RYR2 stabilizers (rycal1 and rycal2) show their antiarrhythmic effects

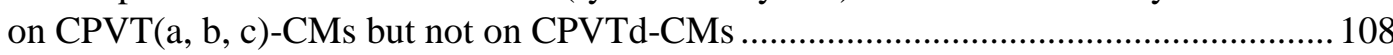

4.4 Differentially regulated cAMP dynamics in Ctrl-CMs and CPVT-CMs ........................ 109

4.4.1 PDE2 and PDE3 activities are decreased in CPVT-CMs ............................................ 110

4.4.2 PDE4 activity shows mutation-specific properties in CPVT-CMs ................................. 111

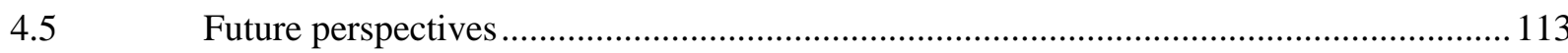

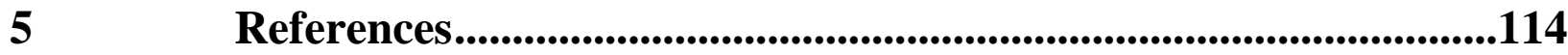

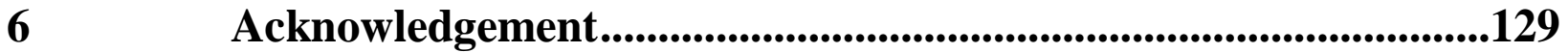




\section{List of abbreviations}

AFP Alpha-1-fetoprotein

ALB Albumin

$\alpha$-MHC Myosin heavy chain (alpha)

AP Action potential

APA Action potential amplitude

APD Action potential duration

APD50 Action potential duration at 50\% repolarization

APD90 Action potential duration at $90 \%$ repolarization

ATP Adenosine triphosphate

$\beta$-AR $\quad \beta$-adrenergic receptor

bFGF Fibroblast growth factor basic

BSA Bovine serum albumin

$\mathrm{Ca}^{2+} \quad$ Calcium

CaMKII $\mathrm{Ca}^{2+} /$ calmodulin-dependent protein kinase II

cAMP 3',5'-cyclic adenosine monophosphate

CASQ2 Calsequestrin 2

CFP Cyan fluorescent protein

cGMP 3'-5'-cyclic guanosine monophosphate

cTNT Cardiac troponin T

CICR Calcium induced calcium release

CMs Cardiomyocytes

CPVT Catecholaminergic polymorphic ventricular tachycardia

Ctrl Control

Cx43 Connexin 43

Cy3 Indocarbocyanine

DAD Delayed afterdepolarization 
DAPI 4', 6-diamidino-2-phenylindole dihydrochloride

DMSO Dimethyl sulfoxide

DPBS Dulbecco's phosphate buffered saline

EAD Early afterdepolarization

EB Embryoid body

ECG Electrocardiogram

EPAC1 Exchange protein directly activated by cAMP 1

ESCs Embryonic stem cells

FBS Fetal bovine serum

FITC Flurorescein isothiocyanate

FKBP12.6 FK506 binding protein

FOXD3 Forkhead box D3

FRET Fluorescence resonance energy transfer

GAPDH Glyceraldehyde-3-phosphate dehydrogenase

GDF3 Growth differentiation factor 3

HCM Hypertrophic cardiomyopathy

hiPSCs Human induced pluripotent stem cells

IBMX 3-isobutyl-1-methylxanthine

ICD Implantable cardioverter-defibrillator

ISO Isoproterenol

JNC Junctin

KLF4 Krüppel-like factor 4

LTCC L-type $\mathrm{Ca}^{2+}$ channel

mAKAP muscle A kinase-anchoring protein

MEA Multielectrode array

MEFs Mouse embryonic fibroblasts

MLC2a Myosin light chain 2, atrial isoform

MLC2v Myosin light chain 2, ventricular isoform 


$\begin{array}{ll}\text { MOI } & \text { Multiplicity of infection } \\ \text { MTG } & \text { Monothioglycerol } \\ \text { NEAA } & \text { Non-essential amino acids } \\ \text { OCT4 } & \text { Octamer-binding transcription factor 4 } \\ \text { PDE2 } & \text { Phosphodiesterase 2 } \\ \text { PDE3 } & \text { Phosphodiesterase 3 } \\ \text { PDE4 } & \text { Phosphodiesterase 4 } \\ \text { PFA } & \text { Paraformaldehyde } \\ \text { PKA } & \text { Protein kinase A } \\ \text { PVC } & \text { Premature ventricular complex } \\ \text { RMP } & \text { Resting membrane potential } \\ \text { RPMI } & \text { Roswell Park Memorial Institute 1640 } \\ \text { RT-PCR } & \text { Reverse transcription PCR } \\ \text { Ryr2 } & \text { Ryanodine receptor 2 (animal) } \\ \text { RYR2 } & \text { Ryanodine receptor 2 (human) } \\ \text { SMA } & \text { Smooth muscle alpha-actin } \\ \text { SOICR } & \text { Store-overload-induced calcium release } \\ \text { SOX2 } & \text { Sex determining region Y-box 2 } \\ \text { STEMCCA Stem cell cassette } \\ \text { SR } & \text { Sarcoplasmic reticulum } \\ \text { TA } & \text { Triggered activity } \\ \text { TH } & \text { Tyrosine hydroxylase } \\ \text { VT } & \text { Ventricular tachycardia } \\ \text { WNT } & \text { Wingless and int } \\ \text { YFP } & \text { Yellow fluorescent protein } \\ & \end{array}$




\section{List of figures}

Figure 1. Typical features of ventricular tachycardia and fibrillation in a patient with CPVT..... 4

Figure 2. Structural domains and accessory proteins of RYR2 ….......................................... 6

Figure 3. Cartoon (top) and schematic representation (bottom) of the RYR2 protein. ................ 8

Figure 4. The cryo-electron microscopy structure of Ryr2 from the porcine heart in a closed

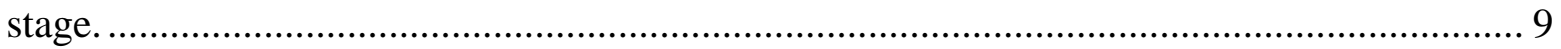

Figure 5. A proposed mechanism for CPVT associated with RYR2 mutations........................ 10

Figure 6. RYR2 mutation causes the disruption of domain-domain interaction. ...................... 11

Figure 7. RYR2 mutation causes the disruption of FKBP12.6 binding.................................... 12

Figure 8. The RYR2 ${ }^{\mathrm{A} 4860 \mathrm{G}}$ mutation causes loss-of-function. ............................................... 13

Figure 9. FRET mechanism (CFP-YFP FRET pair) ...................................................... 21

Figure 10. Principle of the Epac1-cAMP-sensor (Epac1-camps) ........................................ 23

Figure 11 Morphology and alkaline phosphatase staining of generated hiPSCs...................... 46

Figure 12. Gene expression analysis of generated hiPSCs................................................. 47

Figure 13. Immunostaining of pluripotency-related proteins in hiPSCs. ................................. 48

Figure 14. Gene expression analysis of spontaneously differentiated hiPSCs. ......................... 49

Figure 15. Immunostaining of cells during spontaneous differentiation of hiPSCs.................. 50

Figure 16. Teratoma formation of hiPSCs after subcutaneous injection into immunodeficient

RAGC mice.

Figure 17. Diploid karyograms for generated hiPSCs. Respectively showed a normal karyotype

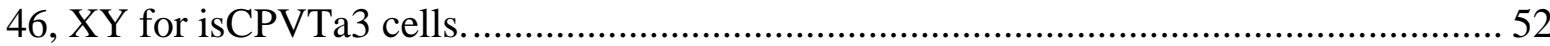

Figure 18. Verification of RYR2 point mutations in CPVTa- and CPVTd-iPSCs..................... 53

Figure 19. Directed differentiation procedures and differentiation efficiency.......................... 54

Figure 20. The differentiated CMs show normal sarcomere structure.................................... 55

Figure 21. Classification of differentiated CMs according to their AP morphologies. ............... 56

Figure 22. Percentage of ventricular-, atrial-, nodal-like and Purkinje-like CMs in Ctrl- and

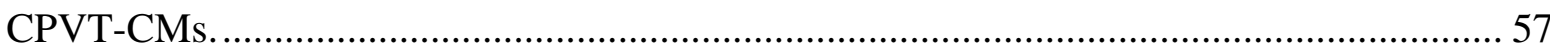

Figure 23. Action potential characteristics of ventricular-like CMs. ....................................... 59

Figure 24. Patch-clamp results show EADs in non-ISO stimulated CPVTd-CMs..................... 61

Figure 25. The MEA technology is used to quantify arrhythmic events. ............................... 62

Figure 26. The representative original traces of MEA recording for Ctrl-CMs, CPVT(a, b, c)-

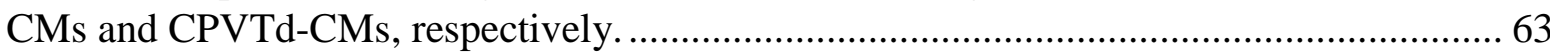

Figure 27. MEA results presented arrhythmic events in non-ISO stimulated CPVTd-CMs....... 65

Figure 28. CPVT-CMs presented DADs, EADs and TAs after ISO stimulation...................... 66

Figure 29. Mutation-specific profiles of DADs, EADs and TAs after ISO stimulation.............. 67

Figure 30. CPVT-CMs generated plenty of PVC-like and VT-like events after ISO stimulation.

Figure 31. Systematic assessment of antiarrhythmic effects of flecainide by patch-clamp

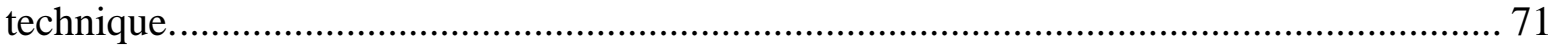

Figure 32. MEA data confirmed antiarrhythmic effects of flecainide for CPVT(a, b, c)-CMs... 73

Figure 33. Systematic assessment of antiarrhythmic effects of dantrolene by patch-clamp technique 
Figure 34. MEA data confirmed antiarrhythmic effects of dantrolene for CPVT(a, b, c)-CMs. . 77 Figure 35. Systematic assessment of antiarrhythmic effects of rycal1 by patch-clamp technique.

Figure 36. MEA data confirmed antiarrhythmic effects of rycal1 for CPVT(a, b, c)-CMs. ....... 81 Figure 37. Systematic assessment of antiarrhythmic effects of rycal2 by patch-clamp technique.

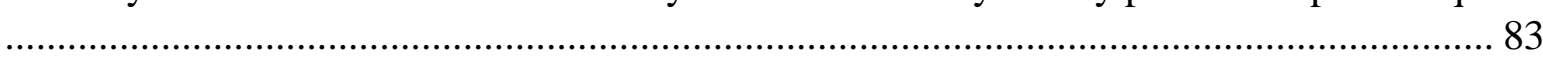

Figure 38. MEA data confirmed antiarrhythmic effects of rycal2 for CPVT(a, b, c)-CMs....... 85 Figure 39. Different FRET changes in Epac1-camps- and Epac1-camps-JNC-transfected CMs induced by different ISO concentrations. ............................................................ 88

Figure 40. cAMP-PDE2 activity in cytosol after $\beta$-adrenergic stimulation. .............................. 89

Figure 41. cAMP-PDE2 activity in the RYR2 compartment after $\beta$-adrenergic stimulation...... 90

Figure 42. cAMP-PDE3 activity in cytosol after $\beta$-adrenergic stimulation. ............................ 91

Figure 43. cAMP-PDE3 activity in the RYR2 compartment after $\beta$-adrenergic stimulation ....... 91

Figure 44. cAMP-PDE4 activity in cytosol after $\beta$-adrenergic stimulation. ............................ 93

Figure 45. cAMP-PDE4 activity in the RYR2 compartment after $\beta$-adrenergic stimulation...... 94 


\section{Abstract}

Catecholaminergic polymorphic ventricular tachycardia (CPVT) is an ion channel disorder in the heart, which is characterized by abnormal calcium handling, ventricular arrhythmias, and sudden cardiac death. This inherited disease is predominantly caused by mutations in the ryanodine receptor type 2 (RYR2). Most of the identified mutations are clustered into four distinct domains of the RYR2 channel. Although heterologous expression systems and animal models have brought important insights in the CPVT pathogenesis, the underlying electrophysiological mechanisms have not been completely understood. The aim of the study was to take cells from CPVT patients with specific RYR2 mutations, to create patient-specific induced pluripotent stem cells (iPSCs), to differentiate these cells into cardiomyocytes (CMs) and then to model the disease in vitro for a better understanding of the disease mechanism and for the investigation of novel therapeutic applications for CPVT patients.

Somatic cells from skin biopsies of CPVT patients carrying the RYR2 mutation in domain a (R420W), domain b (A2254V), domain c (E4076K) or domain d (H4742Y) were isolated and reprogrammed into patient-specific iPSCs. The CPVT as well as healthy control (Ctrl) iPSC lines were differentiated into CMs. The CPVT- and Ctrl-iPSC-derived CMs were investigated for their biological, electrophysiological, and pharmacological differences between the RYR2 mutations in different domains and healthy controls. The differences in 3',5'-cyclic adenosine monophosphate (cAMP) dynamics were investigated as well.

Electrophysiological analyses showed that the CPVT-CMs recapitulated the phenotype of CPVT both by patch-clamp assessment and by multielectrode array assessment. The single CPVTdCMs showed a unique early afterdepolarization (EAD) phenotype in basal condition and isoproterenol- (ISO-) challenged condition. However, CPVTa-, CPVTb- and CPVTc-CMs exhibited delayed afterdepolarization (DAD) and DAD-induced triggered activities (TAs), which were significantly enhanced after the ISO treatment. In the monolayer cultures, all CPVT-CMs revealed a significantly increased number of premature ventricular complex- (PVC-) like events and prolonged duration of ventricular tachycardia- (VT-) like events after the ISO treatment. In contrast, no increased appearance of arrhythmic events (DADs, EADs, DAD- or EAD-induced TAs, and PVC- and VT-like events) was observed in Ctrl-CMs after the ISO treatment. Four 
antiarrhythmic drugs (flecainide, dantrolene, rycal1 and rycal2) showed antiarrhythmic effects on CPVTa-, CPVTb- and CPVTc-CMs, but no or minor antiarrhythmic effect on CPVTd-CMs.

FRET measurement revealed that the contribution of phosphodiesterase 2 (PDE2) to cAMP degradation in all CPVTa- $(15.41 \%)$, b- (9.48\%), c- (15.07\%), d-CMs (7.9\%) were significantly lower than in Ctrl-CMs (27.5\%) in cytosol. The contribution of PDE2 to cAMP degradation at the RYR2 compartment in all CPVTa- (14.19\%), b- (25.21\%), c- (17.32\%), d-CMs (8.6\%) were also significantly lower than in Ctrl-CMs (39.98\%). Similar to PDE2, the contribution of PDE3 to cAMP degradation in all CPVT-CMs were significantly lower than in Ctrl-CMs both in cytosol and at the RYR2 compartment. However, for PDE4, there were lower activities in cytosol in CPVTb- and CPVTc-CMs when compared to Ctrl-CMs, and lower activities at the RYR2 compartment in CPVTc- and CPVTd-CMs when compared to Ctrl-CMs. Furthermore, the data indicate that PDE4 is the major regulator of cAMP level in CPVT-CMs both in cytosol and at the RYR2 compartment after ISO stimulation and that PDE2 and PDE3 have a smaller contribution to regulate the cAMP level.

Taken together, this study reveals that mutation-specific CPVT-iPSCs can be used to model the disease in vitro, to investigate the disease pathophysiological and molecular mechanisms and to optimize drug therapies. 


\section{Introduction}

\subsection{Catecholaminergic polymorphic ventricular tachycardia}

Catecholaminergic polymorphic ventricular tachycardia (CPVT) is induced by emotional stress or physical exercise in patients without structural cardiac abnormalities. This disease was first described as a novel clinical entity by Coumel et al. in 1978 (Coumel et al., 1978) and then in a follow-up study in 1995 (Leenhardt et al., 1995).

\subsubsection{Clinical characteristics}

The electrocardiograms (ECG) of CPVT patients are normal when the patient is at rest, but become polymorphic, bidirectional ventricular arrhythmias after emotional or physical stress (Figure 1). The ventricular arrhythmias sometimes degenerate into ventricular fibrillation, which can lead to syncope or sudden cardiac death. The mean age of occurrence of symptoms (usually a syncopal episode) of CPVT is between seven and twelve years; occurrence as late as the fourth decade of life has been reported. If untreated, CPVT is highly lethal, as approximately $30 \%$ of affected individuals experience at least one cardiac arrest and up to $80 \%$ one or more syncopal spells. Sudden cardiac death may be the first manifestation of the disease (Napolitano. et al., 2014). 


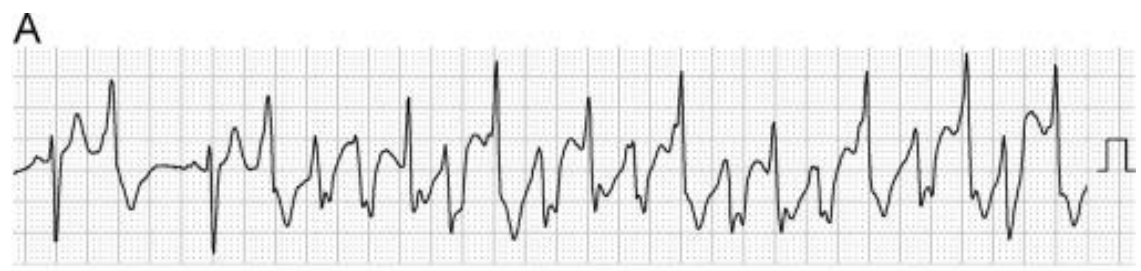

B
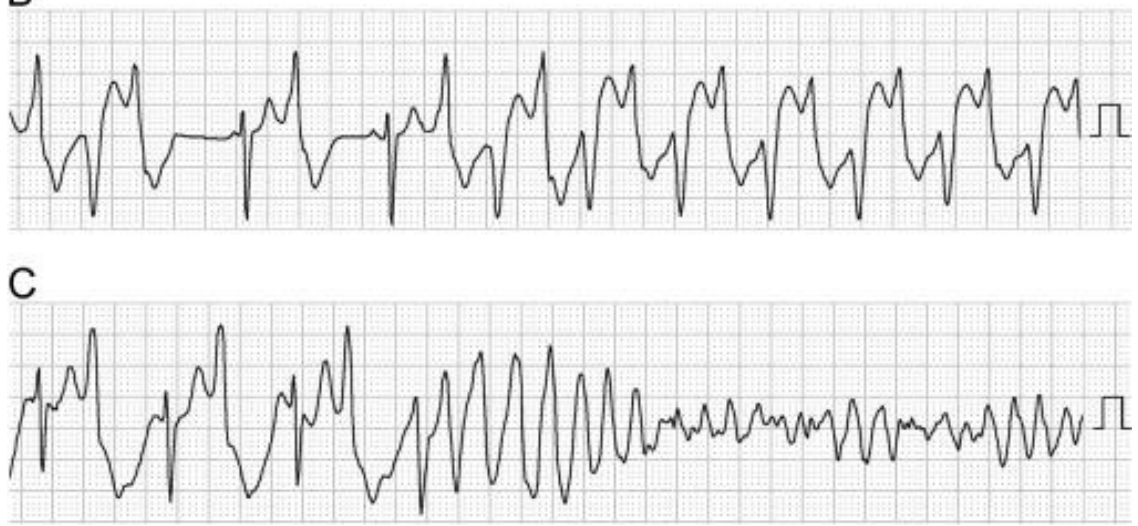

Figure 1. Typical features of ventricular tachycardia and fibrillation in a patient with CPVT. (A) Polymorphic ventricular tachycardia. (B) Bidirectional ventricular tachycardia. (C) Rapid polymorphic ventricular tachycardia deteriorating into ventricular fibrillation. These electrocardiograms were recorded by Holter monitoring in the CM3 lead in the same patient (Sumitomo, 2015). With permission from Elsevier.

\subsubsection{CPVT classification}

Various genotypes of CPVT have been described, ranging from CPVT1 to CPVT5. The most common type of CPVT is caused by a mutation in the ryanodine receptor 2 genes (RYR2) (CPVT1), which accounts for 50\%-55\% of the CPVT cases. The second most common type CPVT2 is caused by a homozygous or compound heterozygous mutation in the calsequestrin 2 gene (CASQ2). Recently, the mutations in the calmodulin gene (CALM1) and triadin gene $(T R D N)$ have been found to be responsible for CPVT4 and CPVT5, respectively. So far, no exact gene has been identified for CPVT3 (Bhuiyan et al., 2007). Based on the inheritance patterns, CPVTs can be divided into two groups: (1) RYR2- and CALMI-related CPVTs are inherited in an autosomal dominant manner, and (2) CASQ2- and TRDN-related CPVTs are inherited in an autosomal recessive manner (Napolitano. et al., 2014) (Table 1). 
Table 1. Subtypes of CPVT

\begin{tabular}{|c|c|c|c|c|c|}
\hline Genes & $\begin{array}{l}\text { Proportion of } \\
\text { all CPVT }\end{array}$ & $\begin{array}{l}\text { CPVT } \\
\text { subtypes }\end{array}$ & $\begin{array}{l}\text { Chromosome } \\
\text { locus }\end{array}$ & $\begin{array}{l}\text { Inheritance } \\
\text { patterns }\end{array}$ & References \\
\hline$R Y R 2$ & $50 \%-55 \%$ & CPVT1 & $1 q 43$ & $\begin{array}{l}\text { Autosomal } \\
\text { dominant }\end{array}$ & (Priori et al., 2002) \\
\hline$C A S Q 2$ & $2 \%-5 \%$ & CPVT2 & $1 \mathrm{p} 13.1$ & $\begin{array}{l}\text { Autosomal } \\
\text { recessive }\end{array}$ & (Lahat et al., 2001) \\
\hline CALMI & $<1 \%$ & CPVT4 & $14 q 32.11$ & $\begin{array}{l}\text { Autosomal } \\
\text { dominant }\end{array}$ & $\begin{array}{l}\text { (Nyegaard et al., } \\
\text { 2012) }\end{array}$ \\
\hline TRDN & Unknown & CPVT5 & $6 q 22.31$ & $\begin{array}{l}\text { Autosomal } \\
\text { recessive }\end{array}$ & $\begin{array}{l}\text { (Roux-Buisson et al., } \\
\text { 2012) }\end{array}$ \\
\hline
\end{tabular}

\subsubsection{RYR2 mutations linked to CPVT}

The RYR2 channel is the largest ion channel, which contains approximately 5000 residues for every homotetramer. Each subunit contains a large cytosolic domain formed by the first $\sim 4300$ $\mathrm{N}$-terminal residues and a smaller transmembrane domain formed by the last $\sim 500 \mathrm{C}$-terminal residues (Priori and Chen, 2011). The transmembrane domain of RYR2 encompasses the channel pore whereas the cytosolic domain contains binding sites for a number of channel regulators (Mohler and Wehrens, 2007). 


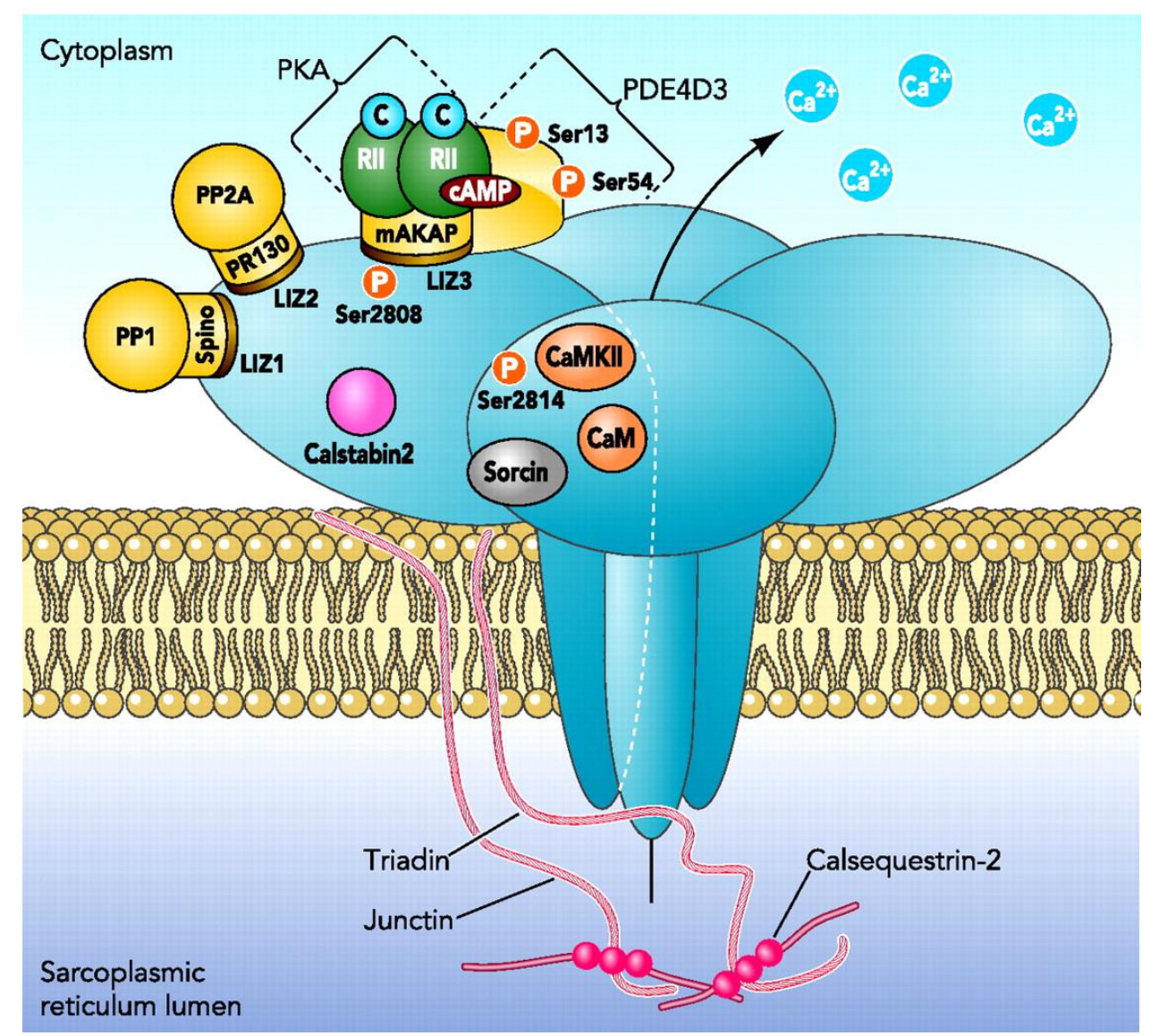

Figure 2. Structural domains and accessory proteins of RYR2. The primary structure of RYR2 and its accessory proteins PP1, PP2A, PKA, calmodulin, and FKBP12.6 are shown. PP1, PP2A and PKA bind to RYR2 via their specific adaptor proteins. CaM, calmodulin; LIZ, leucine-isoleucine zipper (Mohler and Wehrens, 2007). With permission from American Physiological Society.

The sarcoplasmic reticulum (SR) transmembrane proteins triadin and junctin have been associated with the C-terminus of $\mathrm{Ryr} 2$ in the SR lumen, anchoring the $\mathrm{Ca}^{2+}$ binding protein CASQ2 to Ryr2 to form a quaternary complex, termed " $\mathrm{Ca}^{2+}$ release unit" (Kushnir and Marks, 2010). The phosphatases 1 and 2a (PP1 and PP2A), Calstabin 2 (FKBP12.6), protein kinase A (PKA), $\mathrm{Ca}^{2+} /$ calmodulin-dependent kinase II (CaMKII), phosphodiesterase 4D3 (PDE4D3), calmodulin protein $(\mathrm{CaM})$, and sorcin are associated with the N-terminal domain of Ryr2 (Lehnart et al., 2005; Marx et al., 2000; Timerman et al., 1994; Wehrens et al., 2004a). FKBP12.6 is a $12.6 \mathrm{kDa}$ polypeptide that binds to Ryr2 and stabilizes the closed state of the channel. It binds to each Ryr2 subunit separately. On the contrary, PP1 and PP2A and PKA bind to Ryr2 via their specific adaptor muscle A kinase-anchoring protein (mAKAP) (Dodge-Kafka et al., 2005; Scott and Santana, 2010). The cytosolic Ryr2 domain provides leucine/isoleucine 
zipper motifs to facilitate binding of mAKAP. There are three well-studied phosphorylation sites in Ryr2. PKA mainly phosphorylates Ryr2 at serine position 2808 and 2030 (Xiao et al., 2006). In addition, PDE4D3 and phosphatases are targeted to the Ryr2 signaling complex and locally regulate cyclic adenosine monophosphate (cAMP) levels and PKA activity, respectively (Lehnart et al., 2005). CaMKII phosphorylates Ryr2 at serine 2814 (Wehrens et al., 2004a). The phosphorylation of Ryr2 regulates the channel, without directly opening or closing the channel, but modifying the sensitivity of the channel to its physiological ligand $\mathrm{Ca}^{2+}$.

Since 2001, molecular genetic studies have identified mutations in the gene encoding the cardiac RYR2 (Priori et al., 2001) in CPVT patients. These studies have led to the intensification of efforts for elucidating the arrhythmogenic mechanisms underlying the disease. However, due to the protein size and complexity, it is challenging to investigate the topology of mutations.

More than 150 RYR2 mutations for CPVT have been identified until now. The overwhelming majority (more than 76\%) of RYR2 mutations is classified in clusters in four distinct regions: domain a (residues 44-466), b (residues 2246-2534), c (residues 3778-4201), and d (residues 4497-4959) (Figure 3). These regions are highly conserved in RYR2 across species (Priori and Chen, 2011). Most mutations identified in RYR2 are single nucleotide replacements (also called "point mutations") leading to an amino acid substitution, which is different from the other channelopathies where mutations such as truncations, deletions, and insertions are more common. Only a small number of mutations (less than 24\%) are positioned in regions outside the conserved small domains a-d. However, the importance of these domains is not known. 

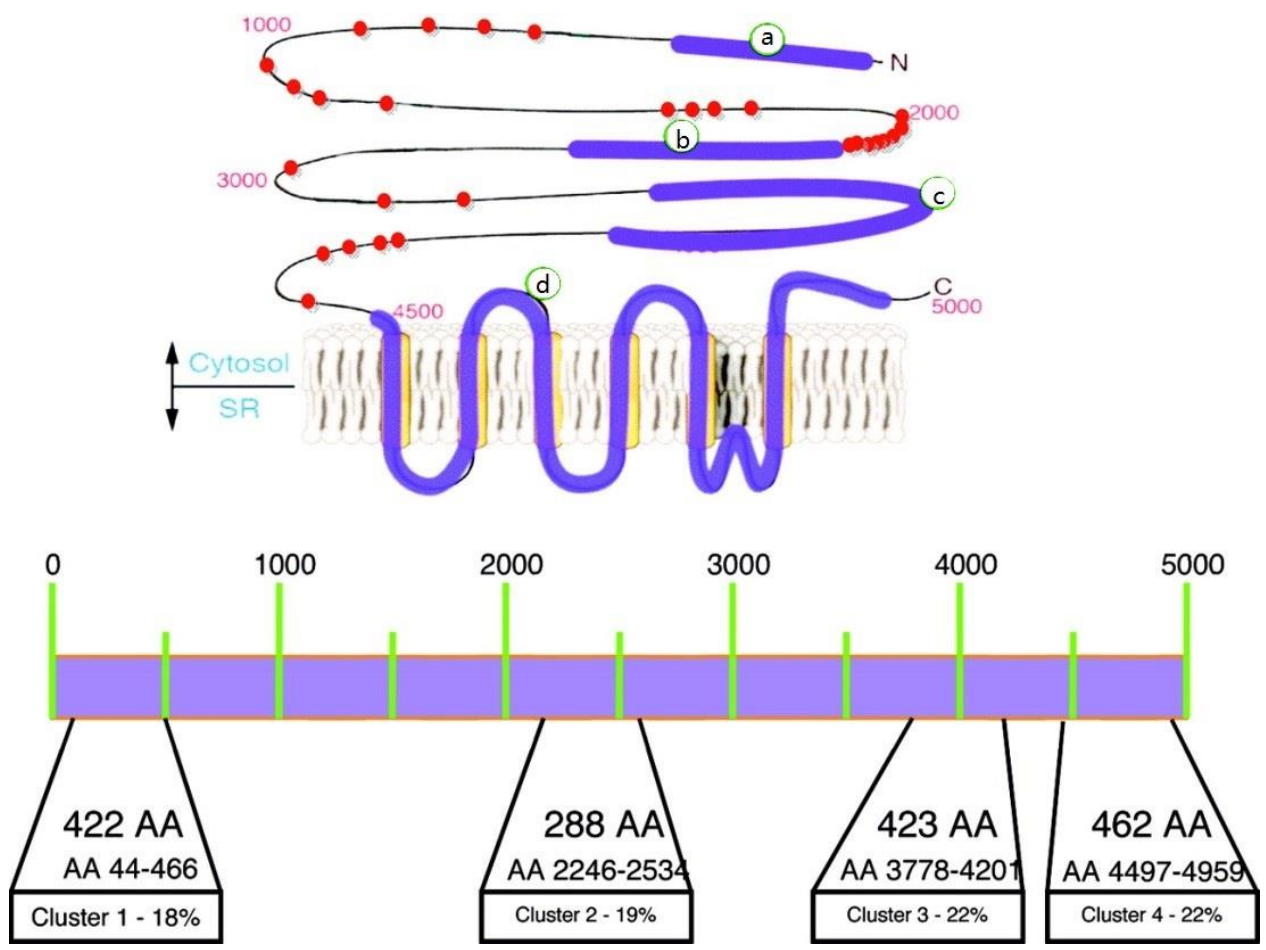

Figure 3. Cartoon (top) and schematic representation (bottom) of the RYR2 protein. Clusters with frequent mutations are depicted with their location along the RYR2 amino acid sequence. Percentage of known (published) mutation for each cluster is also reported (bottom). Mutations outside the canonical clusters are depicted as dots (top). Each dot represents a unique mutation (Priori and Chen, 2011). With permission from Wolters Kluwer Health, Inc.

The structure of this complex protein becomes more clearly thanks to the technology improvements of cryo-electron microscopy. In 2016, the group of Yan has uncovered the structure basis for the gating mechanism of Ryr2 from the porcine heart (Peng et al., 2016). Several domains based on the structure are proposed, including N-terminal domain, SPRY1, phosphorylation site 1, SPRY2, SPRY3, handle domain 1, phosphorylation site 2, handle domain 2 , helical domain, central domain, and channel domain. It is obvious that there are tight overlays for domain a (residues 44-466), b (residues 2246-2534), c (residues 3778-4201), and d (residues 4497-4959) to N-terminal domain (residues 1-642), helical domain 1 (residues 2111-2679), central domain (residues 3613-4207), and channel domain (residues 4486-4968), respectively (Figure 4). 


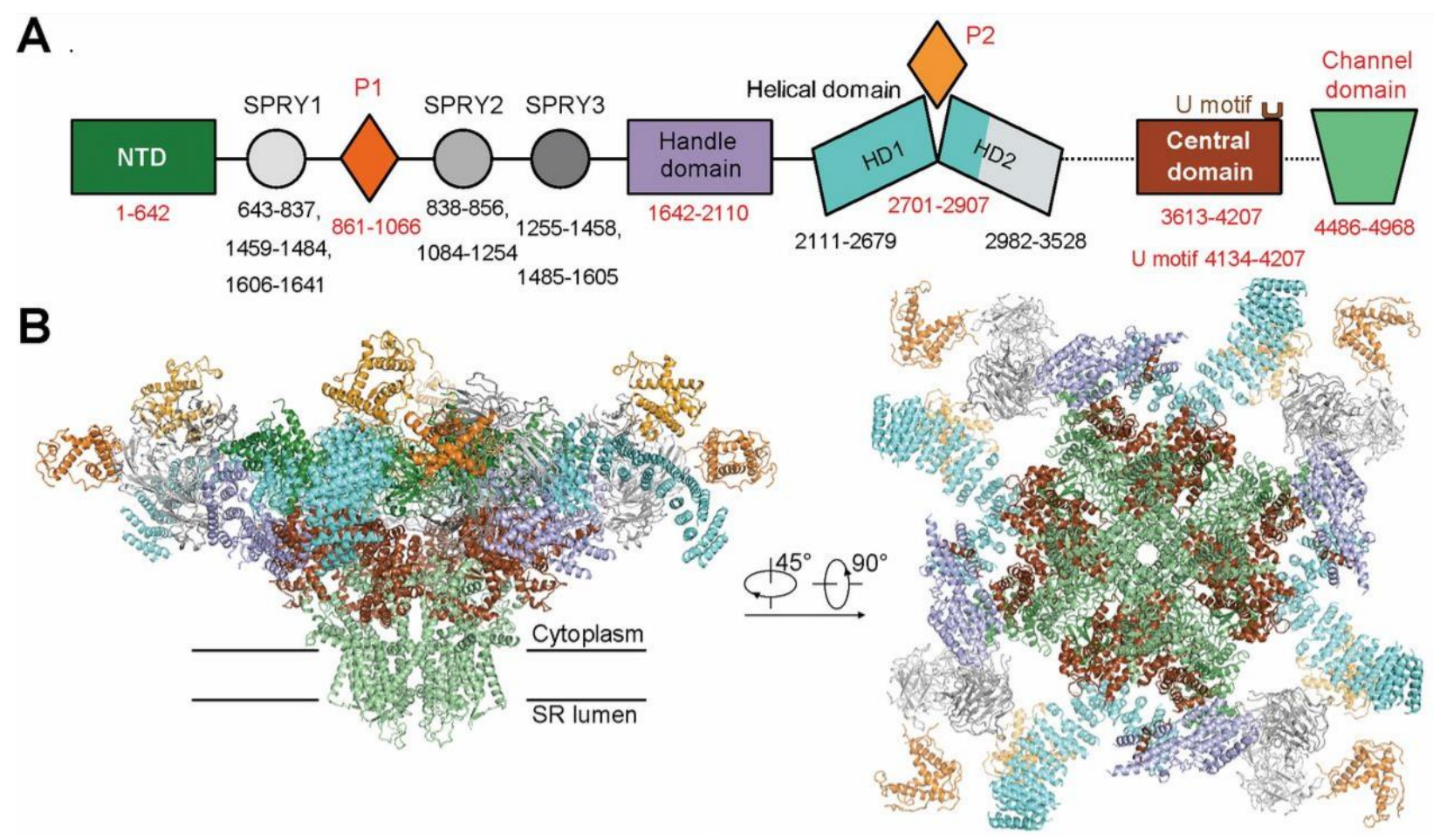

Figure 4. The cryo-electron microscopy structure of Ryr2 from the porcine heart in a closed stage. Domain organization of a porcine Ryr2 protomer $(\mathbf{A})$. The domain boundaries are indicated, with the reliably assigned boundaries labeled red. The tetrameric closed state structure of porcine Ryr2 is domaincolored with the same scheme (B) (Peng et al., 2016). With permission from American Association for the Advancement of Science.

\subsubsection{Pathophysiological mechanisms of CPVT triggered by RYR2 mutations}

It is believed that an interaction of the individual domains is essential for the channel function (Medeiros-Domingo et al., 2009). Nowadays, four hypotheses or mechanisms were proposed to explain the CPVT pathophysiology.

\section{(1) RYR2 mutations cause $\mathrm{Ca}^{2+}$ leak at lower luminal $\mathrm{Ca}^{2+}$ concentration}

This mechanism was first described by the Chen's group in 2004 (Jiang et al., 2004). In normal cardiomyocytes (CMs), an action potential (AP) activates the voltage-dependent L-type $\mathrm{Ca}^{2+}$ channel, leading to a small $\mathrm{Ca}^{2+}$ current influx. This $\mathrm{Ca}^{2+}$ entry induces the RYR2 channel open, resulting in $\mathrm{SR} \mathrm{Ca}^{2+}$ release and muscle contraction. This mechanism is called calcium-induced calcium release (CICR). The free $\mathrm{SR} \mathrm{Ca}^{2+}$ level is predominantly determined by CASQ2. RYR2 primarily determines the threshold for store overload-induced calcium release (SOICR). 
When CPVT CMs are stimulated by catecholamine or stress, an abrupt increase of free SR $\mathrm{Ca}^{2+}$ occurs. In CPVT, the threshold for SOICR is reduced due to the RYR2 mutations. The resting level of free SR $\mathrm{Ca}^{2+}$ in CPVT might have adapted to a reduced level due to the existence of SR autoregulation. Under the stimulation condition, the free $\mathrm{Ca}^{2+}$ level reaches the SOICR threshold, SOICR occurs, leading to a large $\mathrm{SR} \mathrm{Ca}^{2+}$ spillover, which in turn can generate delayed afterdepolarizations (DADs) and triggered arrhythmia (TA) (Jiang et al., 2004) (Figure 5).

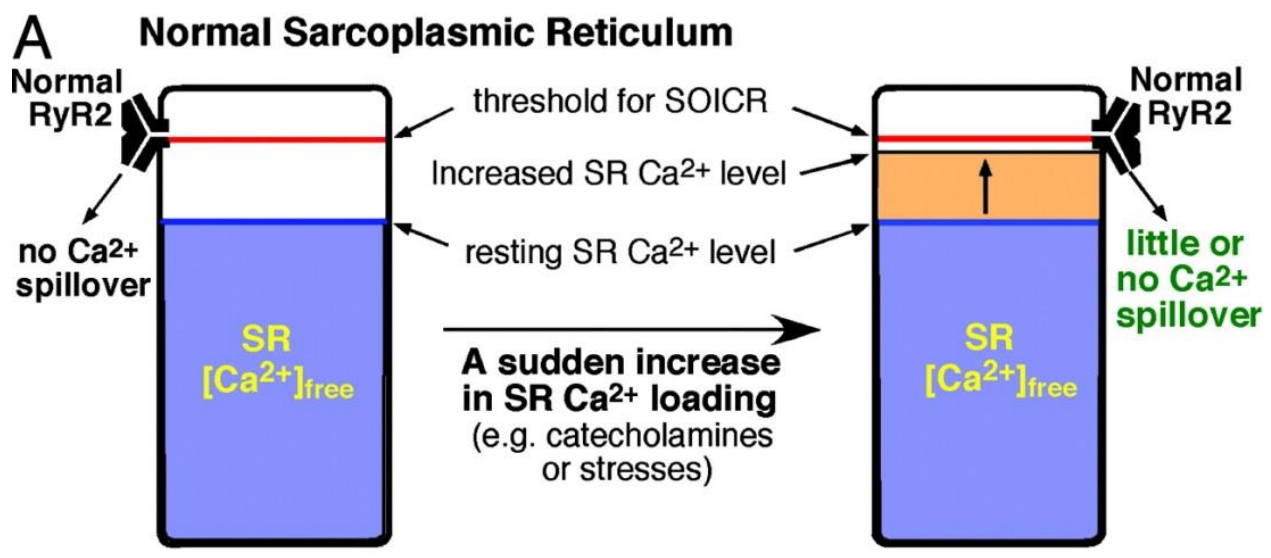

\section{B CPVT Sarcoplasmic Reticulum}

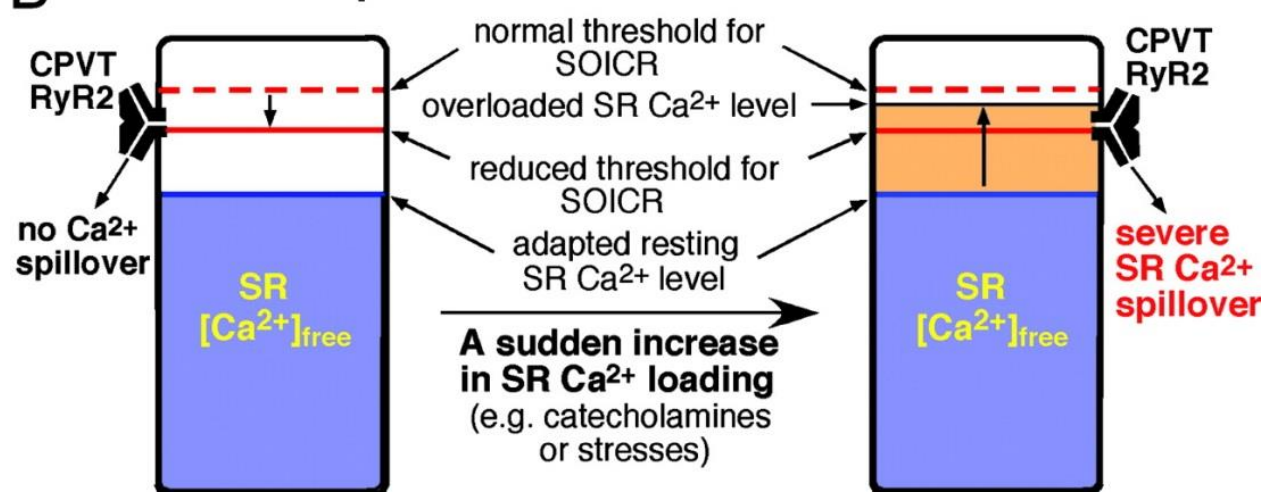

Figure 5. A proposed mechanism for CPVT associated with RYR2 mutations. Schmatic presentation of the relationship between the threshold for SOICR and the free $\mathrm{Ca}^{2+}$ level in normal (A) and CPVT SR (B) in the resting and stimulated states. The threshold for SOICR, which is primarily determined by RYR2, is depicted by a red bar. The threshold for SOICR is reduced in CPVT due to the RYR2 mutations. Blue area represents the free $\mathrm{SR} \mathrm{Ca}{ }^{2+}$ level. The resting level of free $\mathrm{SR} \mathrm{Ca}^{2+}$ in CPVT might have adapted to a reduced level due to the existence of SR autoregulation. An abrupt increase of free $\mathrm{SR} \mathrm{Ca}^{2+}$ is depicted by the yellow area when the cell stimulated by catecholamines or stresses. When the free SR $\mathrm{Ca}^{2+}$ level reaches the SOICR threshold, SOICR occurs, leading to a large $\mathrm{SR} \mathrm{Ca}^{2+}$ spillover, which in turn can generate DADs and TAs (Jiang et al., 2004). Copyright (2004) National Academy of Sciences. 


\section{(2) RYR2 mutations cause the disruption of domain-domain interaction}

This mechanism was first proposed by the Matsuzaki's group in 2011 (Suetomi et al., 2011). In the normal RYR2, the N-terminal domain and the helical domain 1 (Figure 6) interact with each other to function as a regulatory switch for RYR2 channel gating. This interaction forms with a tight "zipping" of the two interacting domains, which serves to stabilize the channel. A mutation in either domain weakens the domain-domain interaction, leading to an unzipping of the two interacting domains, which causes the leakiness of RYR2 channel (Dobrev et al., 2012; Kobayashi et al., 2009; Suetomi et al., 2011) (Figure 6). This hypothesis is supported by using cells co-transfected with single yellow fluorescent protein (YFP) (RYR2S437-YFP) and single cyan fluorescent protein (CFP) (RYR2S2367-CFP) labeled RYR2, indicating that the interaction between the RYR2S437 and RYR2S2367 spots is an inter-subunit interaction (Liu et al., 2010).

\section{Normal RyR2}

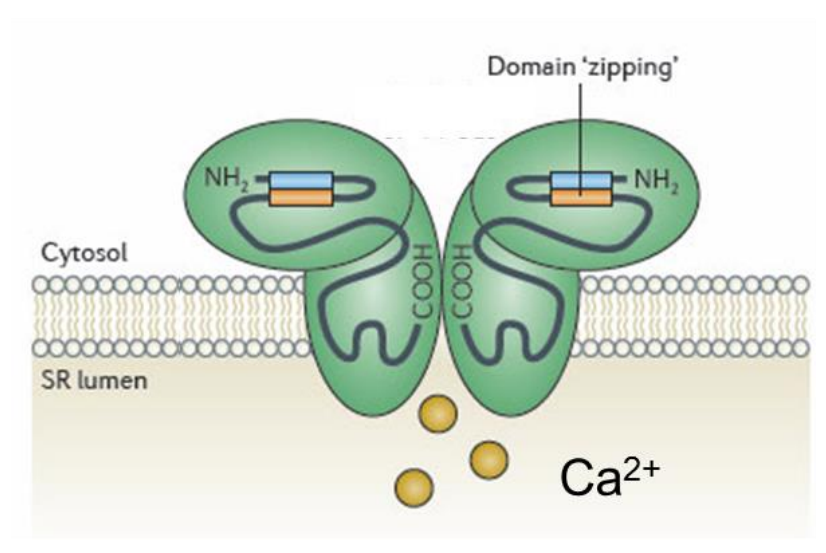

Mutant RyR2

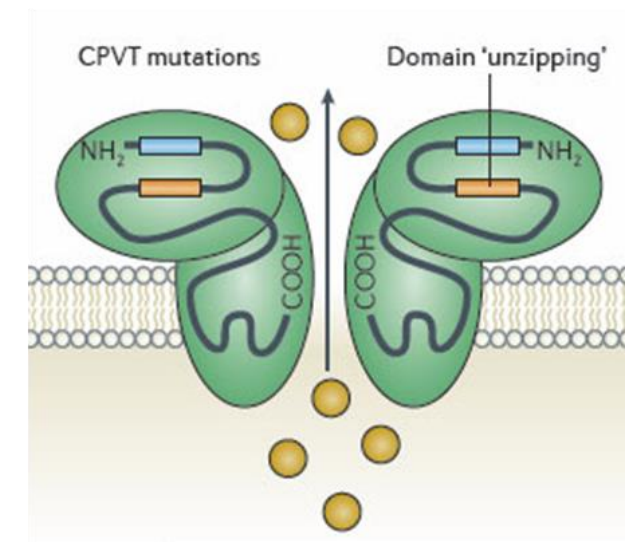

Figure 6. RYR2 mutation causes the disruption of domain-domain interaction. Normal RYR2 function also requires proper interaction between the amino-terminal domain (blue) and a central domain (orange), stabilizes the channel in its closed state, thus preventing diastolic $\mathrm{Ca}^{2+}$ leak from the SR. The RYR2 mutations in CPVT caused amino-terminal domain and central domain unzipping, destabilizing the closed state of RYR2, resulting in diastolic $\mathrm{Ca}^{2+}$ leak from the SR that may activate the $\mathrm{Na}^{+} / \mathrm{Ca}^{2+}$ exchanger to produce DADs and/or TAs (Dobrev et al., 2012). With permisssion from Nature publishing Group.

\section{(3) RYR2 mutations cause the disruption of FKBP12.6 binding}

Andrew Marks suggests that FKBP12.6 acts as a "stabilizer" that maintains the RYR2 channel stability using murine model harboring RYR mutations in helical domain 1 (Figure 4). They 
demonstrate that FKBP12.6 binding to RyR2 R2474S is significantly reduced under adrenergic stimulation. It has been hypothesized that some RYR2 mutants present a reduced binding affinity to FKBP12.6 under basal conditions and this defect is further amplified by PKA phosphorylation of RYR2, which leads abnormal $\mathrm{Ca}^{2+}$ leak from the SR during diastole (Liu et al., 2009; Marx et al., 2000) (Figure 7).

A
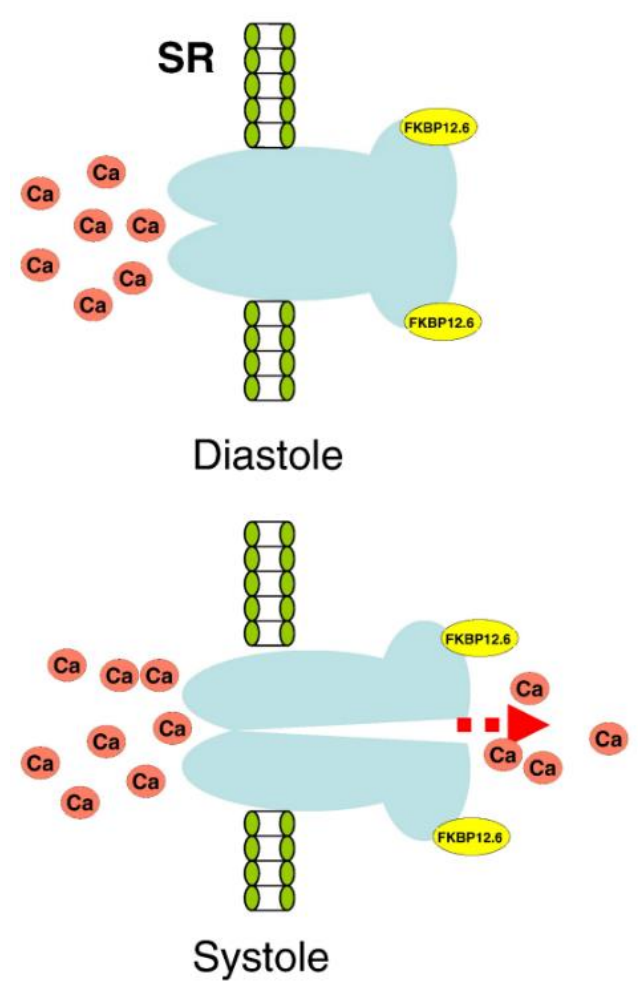

B
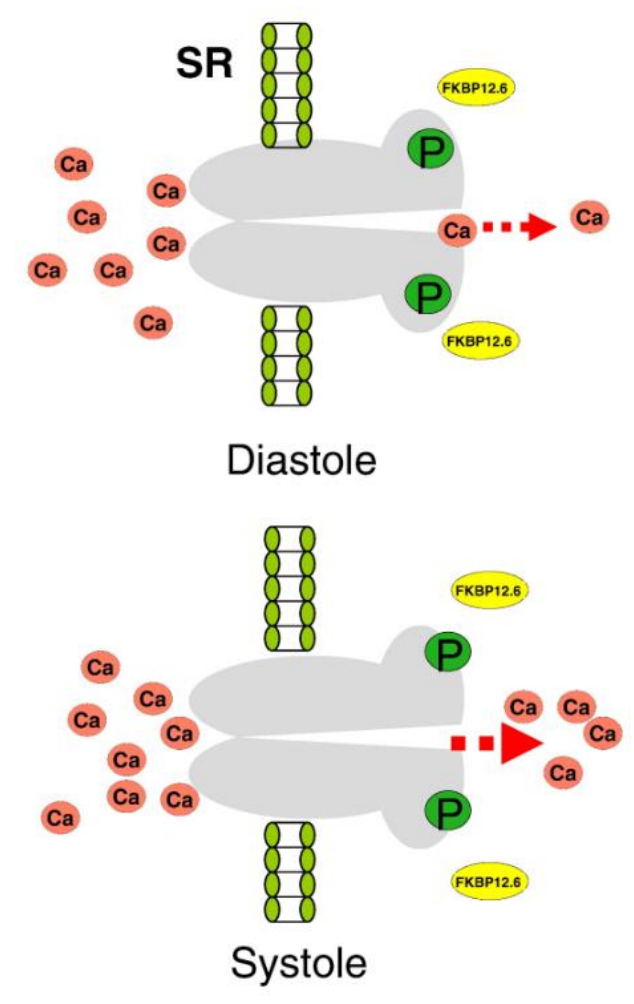

Figure 7. RYR2 mutation causes the disruption of FKBP12.6 binding. (A) The wild-type RYR2 is closed in diastole while during systole it opens. (B) The mutant RYR2 opens during systole and is leaky during diastole. FKBP12.6 (yellow ovals) acts as a "stabilizer" that preserves the RYR2 channel closed during diastole (Liu et al., 2009). With permission from Elsevier.

\section{(4) RYR2 mutations cause loss-of-function of the RYR2 channel}

The three mechanisms mentioned above for CPVT arrhythmogenesis require RYR2 affected by gain-of-function mutations. Recently, Zhao et al. (Zhao et al., 2015) reported a loss-of-function mutation of RYR2 that was originally described in humans, and elucidated novel mechanisms by which hypoactive RYR2 channels trigger malignant arrhythmias. In isoproterenol- (ISO-) 
stimulated ventricular myocytes, the RYR2 loss-of-function mutation decreases the peak of $\mathrm{Ca}^{2+}$ release during systole, gradually overloading the SR with $\mathrm{Ca}^{2+}$. The resultant $\mathrm{Ca}^{2+}$ overload then randomly causes bursts of prolonged $\mathrm{Ca}^{2+}$ release, inducing electrogenic $\mathrm{Na}^{+} / \mathrm{Ca}^{2+}$ exchanger activity and triggering EADs in systole (Figure 8).
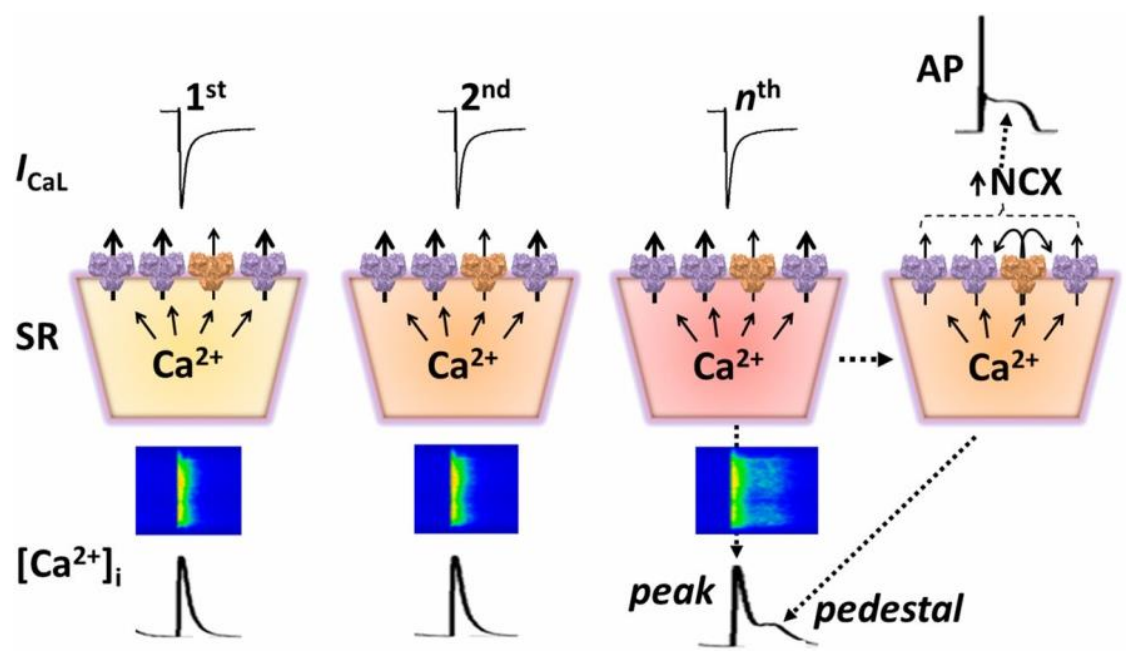

Figure 8. The RYR2 ${ }^{\mathrm{A} 4860 \mathrm{G}}$ mutation causes loss-of-function. In a single $R Y R 2^{\mathrm{A} 4860 \mathrm{G}} \mathrm{CM}$ containing functionally divergent RYR2 channels (WT shown in violet, mutation shown in bronze), a normal Ltype calcium current first elicits a $\left[\mathrm{Ca}^{2+}\right]_{i}$ transient. The lower amplitude of the $\left[\mathrm{Ca}^{2+}\right]_{i}$ transient leaves a residual amount of $\mathrm{Ca}^{2+}$ inside the $\mathrm{SR}$, which gradually builds up. This process is repeated $\mathrm{N}$ times until the SR $\mathrm{Ca}^{2+}$ load reaches a threshold that is sufficient to activate hyporesponsive RYR2 channels, which then release $\mathrm{Ca}^{2+}$ and further activate neighboring RYR2 channels, enhancing calcium-induced calcium release. The activation of hyporesponsive RYR2 channels immediately after an initial burst of peak $\mathrm{Ca}^{2+}$ release generates a pedestal phase of $\mathrm{Ca}^{2+}$ release that supercharges the sarcolemmal $\mathrm{Na}^{+} / \mathrm{Ca}^{2+}$ exchanger, generating an EAD (Zhao et al., 2015). Copyright (2015) National Academy of Science.

\subsubsection{Current therapies for CPVT}

Patients with CPVT are generally recommended to avoid overwhelming emotions, strenuous exercise, and competitive sports. First-line therapy for CPVT is $\beta$-blockers combined with exercise restriction. Nadolol, a long-acting, non-selective $\beta$-blocker, is preferred for prophylactic treatment of CPVT. Propranolol, another non-selective $\beta$-blocker, is also an effective medication. The third generation of $\beta$-blocker carvedilol, which is a non-selective $\beta$-blocker and $\alpha 1$-blocker, is reported to inhibit the arrhythmic events in CPVT. However, $\beta$-blockers cannot completely suppress the arrhythmic events in CPVT patients. Probably due to differences in $\beta$-blocker 
dosages or a poor drug compliance or polymorphisms, the efficacies of $\beta$-blocker treatment are apparent discrepancy among various studies (Leenhardt et al., 2012).

Flecainide acetate is a class Ic antiarrhythmic agent used to prevent and treat tachyarrhythmias. The efficacy of flecainide is first observed in a CPVT CASQ2 knockout mouse model, showing a reduction of ventricular arrhythmia both in vitro and in vivo through Ryr2 blockage (Watanabe et al., 2009). Routine use of flecainide monotherapy is not recommended because the evidence is scarce, despite the side effects of flecainide are mild in contrast to $\beta$-blockers (Lieve et al., 2016). The proposed underlying mechanism of the action of flecainide is controversial. Apart from the known sodium channel blocking effect, flecainide has an additional uncertain direct blocking effect on the RYR2 channel (Bannister et al., 2015).

Implantable cardioverter-defibrillators (ICDs) are recommended for patients with CPVT who experience cardiac arrest, recurrent syncope or ventricular tachycardia (VT) despite $\beta$-blockers and flecainide medical therapy. Furthermore, in those individuals for whom the highest tolerated dose of $\beta$-blockers fails to adequately control arrhythmias (Priori, 2002), an ICD can be the primary prevention of cardiac arrest/sudden death. However, there are significant drawbacks to ICD therapy, because several case reports have shown the potential proarrhythmic properties of the ICD. An ICD shock may cause a subsequent catecholamine release, which in turn can trigger more ventricular arrhythmias and lead to a ventricular arrhythmic storm (Lieve et al., 2016)

Left cardiac sympathetic denervation is used to treat angina pectoris, long-QT syndrome and highly lethal acute myocardial infarction (van der Werf et al., 2012b). This surgery may be considered for those CPVT patients who have several appropriate ICD shocks while on $\beta$ blocking agents and for those who are intolerant or have contraindication to $\beta$-blocker therapy (Priori et al., 2013). However, left cardiac sympathetic denervation have many side effects, including palpebral ptosis, elevation of the left hemidiaphragm, and lack of sweating from the left arm and face. Pharmacologic therapy should be always optimized prior to considering this therapy, also due to recurrences of cardiac arrhythmic events that have been reported in those with this therapy (Napolitano. et al., 2014).

\subsection{Application of human induced pluripotent stem cells in cardiac research}




\subsubsection{Human induced pluripotent stem cells}

Stem cells are characterized by the ability to self-renew and to generate differentiated functional cell types (Jaenisch and Young, 2008). Since pluripotent stem cells have the greatest developmental and therapeutic potential, they have been intensely investigated for more than six decades. Embryonic stem cells (ESCs) were first derived from the inner cell mass of mouse blastocysts (Evans and Kaufman, 1981) and subsequently from human embryos (Thomson et al., 1998).

Obtaining and isolation of human ESCs means destruction of the embryo, which causes critical political and ethical problems. Therefore, the possibility of reprogramming differentiated somatic cells and setting them back in a pluripotent state provides a powerful tool for developmental and clinical research without ethical problems.

In 2006, Takahashi and Yamanaka achieved a significant breakthrough in reprogramming somatic cells back to an ESC-like state in mouse model (Takahashi and Yamanaka, 2006). They successfully reprogrammed mouse embryonic fibroblasts (MEFs) and adult fibroblasts to pluripotent ESC-like cells, so-called induced pluripotent stem cells (iPSCs), after viral-mediated transduction of the four transcription factors OCT3/4 (octamer-binding transcription factor 3/4), SOX2 (sex determining region Y-box 2), $c-M Y C$, and KLF4 (Krüppel-like factor 4). Subsequently, human iPSCs were generated from fibroblasts using the same combination of transcription factors (Takahashi et al., 2007) using retroviruses or using different combinations like OCT4 (Octamer-binding transcription factor 4), SOX2, NANOG and LIN28 (Yu et al., 2007) using lentiviral vectors.

Retrovirus and lentivirus are potentially harmful genome-integrating viruses. This leads to insertion mutations, which for instance could lead to diverse mutations or even cell death if an essential gene is affected. Regarding the transgenic integration and genomic instability, non-viral and non-integrating methods have been explored. There are examples of integration-free vector systems: adenoviruses (Stadtfeld et al., 2008), Sendai viruses (Fusaki et al., 2009), and plasmids (Okita et al., 2011) as well as proteins (Zhou et al., 2009) or even chemicals (Hou et al., 2013). Besides the reprogramming methods, different sources or cell types have been applied, including widely used fibroblasts, urine (Zhou et al., 2012), amniotic fluid (Galende et al., 2010), dental 
pulp cells (Tamaoki et al., 2010), keratinocytes (Streckfuss-Bomeke et al., 2013) and bone marrow cells (Streckfuss-Bomeke et al., 2013).

Human iPSCs (hiPSCs) resemble human ESCs in defining features like morphology, expression of pluripotency markers, self-renewal, and teratoma formation. However, hiPSCs might retain epigenetic memory of the somatic cells of origin (Vaskova et al., 2013), making it more difficult to differentiate to specific cell types. Long-term culture of iPSCs diminish the epigenetic memory of differentially methylated regions, implying that iPSCs lose the characteristics inherited from the parent cells and adapt to very closely resemble ESCs over time (Nishino et al., 2011).

\subsubsection{Direct differentiation of hiPSCs into CMs}

In early studies using pluripotent stem cells as a source for cardiac cells, the embryoid body (EB) method was applied, but resulting in very low CM differentiation efficiency. Here, the pluripotent cells are cultivated in suspension in small aggregates, which results in spontaneous but not directed differentiation into cells of all three germ layers (Amit and Itskovitz-Eldor, 2002).

The differentiation into mesodermal cells is mainly regulated by transforming growth factor type $\beta$ (TGF $\beta$ ), bone morphogenic protein, fibroblast growth factor (FGF) and WNT (derived from the combination of both names wingless and int). The canonical WNT pathway is crucial for cardiac development. Recently, it is reported that modulation of canonical WNT/ $\beta$-catenin signaling under fully defined conditions in iPSCs is sufficient for directed differentiation into CMs (Lian et al., 2012). The iPSCs were first differentiated into mesendoderm cell by the inhibition of glycogen synthase kinase 3 and then into cardiac mesoderm by the inhibition of Wnt signaling pathways. The protocols Lian et al. developed are sufficient to yield a high virtually purity (80-98\%) of functional CMs in 14 days from multiple hiPSC lines without cell sorting or selection (Lian et al., 2013). Furthermore, by changing the $\mathrm{B} 27^{\mathrm{TM}}$ supplemented basal medium RPMI 1640 to a chemically defined medium consisting of just three components: the basal medium RPMI 1640, L-ascorbic acid 2-phosphate and rice-derived albumin (recombinant human), Burridge et al. developed an optimized cardiac differentiation strategy. The authors 
reported that this protocol produced contractile sheets of up to 95\% cardiac troponin T-positive CMs at a yield of up to $100 \mathrm{CMs}$ for every input pluripotent cell and was effective in 11 hiPSC lines tested (Burridge et al., 2014).

\subsubsection{Disease modeling for CPVT}

Ebert et al. firstly conducted their research on spinal muscular atrophy using hiPSCs to model genetically inherited disease to study the specific pathology characters (Ebert et al., 2009). In the past 10 years, an increasing number of studies were reported, in which patient-specific hiPSCs were used for modelling cardiovascular diseases, including Long QT syndrome type 1 (Egashira et al., 2012; Moretti et al., 2010; Sogo et al., 2016), type 2 (Itzhaki et al., 2011; Lahti et al., 2012; Matsa et al., 2011), type 3 (Malan et al., 2011; Terrenoire et al., 2013), familial hypertrophic cardiomyopathy (HCM) (Lan et al., 2013), Timothy syndrome (Yazawa et al., 2011), CPVT (Di Pasquale et al., 2013; Fatima et al., 2011b; Itzhaki et al., 2012; Jung et al., 2012; Kujala et al., 2012; Novak et al., 2015; Novak et al., 2012; Penttinen et al., 2015; Preininger et al., 2016; Zhang et al., 2013a), and dilated cardiomyopathy (DCM) (Gramlich et al., 2015; Sun et al., 2012). The generation of disease-specific CMs differentiated from hiPSCs (hiPSC-CMs) provides a tool for the identification of the pathophysiological and molecular mechanisms in cardiac channelopathies. Despite iPSC-CMs are still immature in comparison to adult myocytes, this technology overcomes the impossibility of harvesting adult human CMs from patients and expanding them in culture.

Dozens of literature focused on CPVT disease modeling have been published nowadays. Two hiPSC models with two mutations in N-terminal domain of RYR2 are reported, one is S406L (Jung et al., 2012) and the other is R420Q (Novak et al., 2015). The authors prove the defective inter-domain interactions within the RYR2 channel as the pathomechanism of the S406L mutation by using dantrolene to restore normal $\mathrm{Ca}^{2+}$ spark properties and to rescue the arrhythmogenic phenotype (Jung et al., 2012). By using RYR2 $2^{\mathrm{R} 420 \mathrm{Q}}$ as a representative for CPVT1 and CASQ2 ${ }^{\mathrm{D} 307 \mathrm{H}}$ mutation for CPVT2, the authors compared the similarities/differences in ultrastructure, calcium handling and other aspects between RYR2 versus CASQ2 mutations underlying CPVT1 and CPVT2 syndromes (Novak et al., 2015). There are three reports of 
RYR2 mutations E2311D (Di Pasquale et al., 2013) and F2483I (Fatima et al., 2011a; Zhang et al., 2013a), which located in helical domain 1 of RYR2 (Figure 4). An antiarrhythmic drug KN93 drastically reduces the presence of DADs and rescues the arrhythmic phenotype induced by

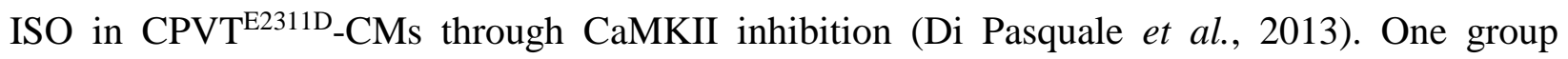
reports that RYR2 ${ }^{\mathrm{F} 2483 \mathrm{I}}$ mutant hiPSC-CMs have aberrant unitary $\mathrm{Ca}^{2+}$-signaling, smaller $\mathrm{Ca}^{2+}$ stores, higher CICR gain (Zhang et al., 2013a). The other group reports that RYR2 ${ }^{\mathrm{F} 2483 \mathrm{I}}$ hiPSCCMs exhibit DADs and higher amplitudes and longer durations of spontaneous $\mathrm{Ca}^{2+}$ release events at basal state (Fatima et al., 2011a). Itzhaki et al. showed that flecainide could eliminate all afterdepolarizations induced by ISO in hiPSC-CMs with the RYR2 ${ }^{\mathrm{M} 4109 \mathrm{R}}$ mutation (located in central domain). Importantly, the threshold for SOICR is significantly reduced in the CPVT $^{\mathrm{M} 4109 \mathrm{R}}$ CMs (Itzhaki et al., 2012). In 2016, Preininger et al. provide new evidence that iPSC-CMs can recapitulate patient-specific drug responses to $\beta$-blockers, by testing flecainide and $\beta$-blocker on the iPSC-CMs harboring novel mutation RYR2 ${ }^{\mathrm{L} 3741 \mathrm{P}}$. The mutation L3741P locates in the central domain of RYR2 based on the discovery of latest structure (Figure 4). Recently, a systemic CPVT investigation is using recruited patients and iPSC-CMs to assess various mutations distributed in different mutation hotspots and to test the antiarrhythmic effects of dantrolene (Penttinen et al., 2015). Dantrolene reduces the number of premature ventricular complexes (PVC) on average by 74\% (range 33-97\%) in four patients with RYR2 mutations in helical domain 1 (P2328S, T2538R, and L4115F) in the cytosolic region of the RYR2 protein, while dantrolene has no effect in two patients with RYR2 mutations in transmembrane domain (V4653F) or central domain (Q4201R) (Penttinen et al., 2015). The similar results are obtained in iPSC-CMs with RYR2 mutations in the helical domain 1 (P2328S, T2538R, and L4115F), dantrolene suppress the $\mathrm{Ca}^{2+}$ cycling abnormalities in $80 \%$ (range $65-97 \%$ ) of cells while the other mutations only in 23 or $32 \%$ of iPSC-CMs with mutations transmembrane domain (V4653F) or central domain (Q4201R) (Penttinen et al., 2015).

\section{3 cAMP dynamic in cardiomyocytes}

cAMP is a very important second messenger in many biology processes. In the mammalian heart, cAMP is the main second messenger for $\beta$-adrenergic receptor ( $\beta$-AR) signaling to control 
chronotropic, inotropic and lusitropic effects (Boularan and Gales, 2015). Catecholamineinduced activation of $\beta$-ARs via adrenaline or noradrenaline leads to the regulation of intracellular cAMP production in CMs. Recent cardiovascular research shows that three different types of $\beta$-ARs are expressed in mammalian hearts: $\beta 1$-, $\beta 2$ - and $\beta 3$-ARs (Imbrogno et al., 2015). The $\beta 1$-AR represents $70-80 \%$ of total $\beta$-ARs in the non-aged heart.

The PKA phosphorylation of several downstream targets, which include L-type $\mathrm{Ca}^{2+}$ channel (LTCC), phospholamban, RYR2, and troponin I, results in enhanced calcium current, increased $\mathrm{Ca}^{2+}$ reuptake mediated by sarco/endoplasmic reticulum $\mathrm{Ca}^{2+}$-ATPase $2 \mathrm{a}$, increased $\mathrm{Ca}^{2+}$ release from the SR, and altered $\mathrm{Ca}^{2+}$ sensitivity of the myofilaments to increase cardiac performance and output (Bers, 2002).

Phosphodiesterases (PDEs) play an essential role in cAMP dynamic by degrading cAMP. There are 11 families of distinct mammalian PDEs based on sequence homology, enzymatic properties, and sensitivity to inhibitors (Omori and Kotera, 2007; Zaccolo, 2006). However, at least six different PDE families are expressed in animal hearts, including PDE1-5 and PDE8 (Miller and Yan, 2010; Xiang, 2011).

\subsubsection{Altered cAMP dynamic in cardiovascular disease}

PDE2 and PDE3 have dual substrate specificity and can hydrolyze both cAMP and 3'-5'-cyclic guanosine monophosphate (cGMP), whereas PDE4 hydrolyses cAMP exclusively. The role of PDE2 in cardiac physiology in vivo has not been well characterized, although several previous reports focus on the LTCC effected by PDE2 hydrolization via NO/cGMP singling pathway (Dittrich et al., 2001; Mongillo et al., 2006; Rivet-Bastide et al., 1997). It is reported that PDE2 activity was up-regulated in response to pressure overload of rat ventricles under pathological conditions, but the absence of significant changes in PDE2A mRNA levels indicated that the PDE2 activity was post-transcriptionally up-regulated (Yanaka et al., 2003). In failing human hearts, an increased amount of AKAP-bound PDE2 may prevent PDE2 from hydrolyzing cyclic nucleotides, and contribute to the pathology of heart failure (Aye et al., 2012). Recently, Mehel et al. reported that PDE2 was up-regulated in human failing hearts and desensitized against acute $\beta$-AR stimulation (Mehel et al., 2013). Antihypertrophic effect of PDE2 has also been reported 
(Zoccarato et al., 2015). Amrinone, milrinone and enoximone, which are known as PDE3 inhibitors, have been used to treat congestive heart failure (Baim et al., 1983). Several clinical trials have revealed that long-term treatment with PDE3 inhibitors in severe chronic heart failure patients is associated with increased mortality and mobility due to arrhythmias and sudden cardiac death (Packer, 1989; Packer et al., 1991). Loss of cardiac efficacy of PDE3 inhibitors has been proposed to associate with disease-related reduction of PDE3 function in several early studies by using left ventricular muscles from patients with various stages of heart failure. Moreover, Chen et al. have demonstrated that the overall PDE3 activity and PDE3A protein levels were significantly reduced in left ventricular myocardial samples from patients with endstage DCM and ischemic heart disease (Ding et al., 2005; Yan et al., 2007). The decreasing of PDE3 activity and PDE3 protein levels in left ventricular endocardium has also been observed in pacing-induced failing canine hearts (Sato et al., 1999). Two similar researches demonstrate a $50 \%$ to $59 \%$ reduction of PDE3A/GAPDH ratios in both left and right ventricular of pacing-induced canine heart failure model (Smith et al., 1998; Smith et al., 1997). Consistent with PDE3A mRNA levels, PDE3A protein was decreased in the canine failing hearts in those two studies. Interestingly, Wu et al. reported that PDE2A and 3A were upregulated in CMs in an iPSC model of DCM via epigenetic regulation (Wu et al., 2015).

PDE4A, 4B, and 4D are expressed in the heart (Abi-Gerges et al., 2009; Galindo-Tovar and Kaumann, 2008; Lehnart et al., 2005; Leroy et al., 2011; Mika et al., 2014; Molina et al., 2012; Takahashi et al., 2002; Xiang et al., 2005), which are highly specific for cAMP. PDE activity assays reveal that PDE4 is responsible for approximately $10 \%$ of total cAMP hydrolytic activity in human, approximately 30\% in mouse, and 50-60\% in rat (Richter et al., 2011). Studies in murine heart with genetic depletion of PDE4B and PDE4D reveal that these subtypes are important for normal regulation of CICR in CMs, and depletion of any of them can cause arrhythmia upon transient $\beta$-adrenergic stimulation (Leroy et al., 2011).

Moreover, the PDE4D3 isoform is reported to interact with the RYR2 complex via mAKAP (Dodge-Kafka et al., 2005; Dodge et al., 2001) and acts as a key regulator of PKA-mediated RYR2 phosphorylation (Lehnart et al., 2005). Both knockout and inhibition of PDE4D in the complex resulted in RYR2 hyperphosphorylation and $\mathrm{SR} \mathrm{Ca}^{2+}$ leakage, and finally catecholaminergic arrhythmogenesis (Lehnart et al., 2005). This is also confirmed by PDE4D inhibition in human atrial heart tissue (Molina et al., 2012). 


\subsubsection{Fluorescence resonance energy transfer}

Fluorescent resonance energy transfer (FRET) describes the interaction between a donor fluorophore, initially in its excited state, which may transfer energy to an acceptor fluorophore through non-radiative energy transfer way (Förster, 1948).

These electrons are lifted from the unexcited single state S0 to its excited state S1 after absorption of photon energy. Due to the emission spectrum of the donor fluorophore overlapping with the excitation spectrum of the acceptor fluorophore, the acceptor fluorophore is excited to S1 with a close proximity of less than $10 \mathrm{~nm}$ (Wu and Brand, 1994). The acceptor electrons are lifted from S0 to the S1 state after absorbing the energy emitted by the acceptor fluorophore. Again, fluorescence is emitted as the acceptor electrons return to the S0 state (Figure 9).

A

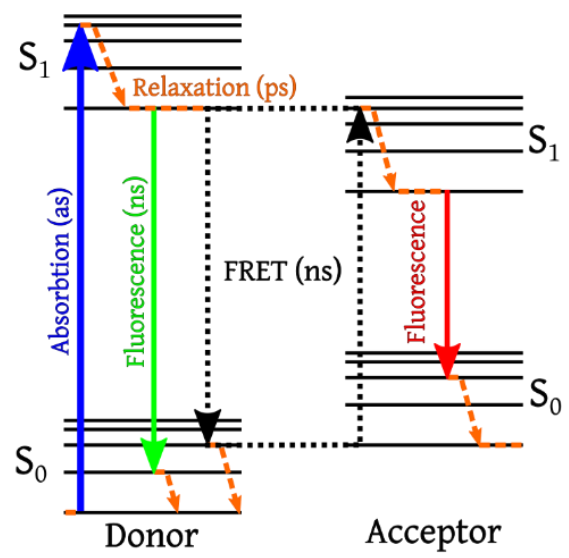

B

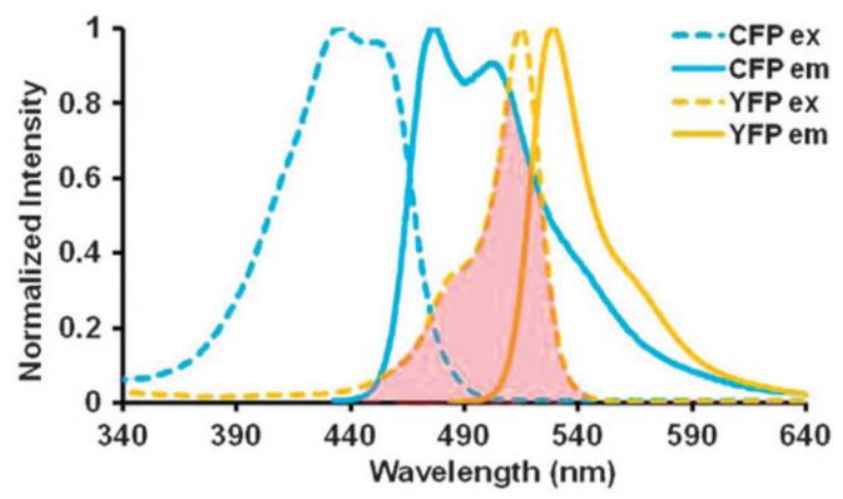

Figure 9. FRET mechanism (CFP-YFP FRET pair). (A) Schematic illumination of the Jablonskidiagram: after absorption of photon energy, the donor electrons are transitioned from a ground state (S0) to an excited state (S1). The donor fluorophore can partly transfer its energy from its lowest S1 level to the electrons of an acceptor fluorophore with a lower energetic excitation spectrum (fluorophore distance must be $\leq 10 \mathrm{~nm}$ ). Acceptor electrons are lifted from their S0 to the $\mathrm{S} 1$ level and fluorescence (hv) is emitted when the acceptor electrons "fall back" into their S0 level. (B) Shown is the needed grey colored spectral overlap, here CFP and YFP as an example. The emission of the donor fluorophore (CFP) and the absorption spectrum of the acceptor YFP overlaps to allow fluorescence resonance energy transfer (Wang and Wang, 2009). With permission from Royal Society of Chemistry. 


\subsubsection{FRET-based cAMP biosensors}

Cyclic AMP content in all cell types or tissues can be traditionally measured by standard antibody-based techniques such as radioimmunoassays and enzyme-linked immunoassays (Brooker et al., 1979; Williams, 2004). These methods allow quantification of total cAMP content (free cytosolic cAMP plus cAMP bound to various proteins) after lysis or mechanical disruption of the tissue.

In the past decades, the FRET technique has gained great popularity in the study of biochemical events and protein-protein interactions in life science, especially in cAMP dynamic. Traditional methods have no spatial resolution and do not allow measurements of physiologically relevant, free cAMP concentrations in intact living cells or in various subcellular locations within one cell. In 1991, Adams et al. created the pioneer FRET-based cAMP sensor, named FlCRhR. This probe consists of a PKA holoenzyme with two fluorescent dyes chemically fused to the R-and C-subunits of PKA and needs to be microinjected into living cells to monitor PKA dissociation upon elevation of intracellular cAMP and its re-association when cAMP is decreased.

Up to now, a series of genetically encoded cAMP biosensors have been generated, which are easily introduced into cells by transfection of the DNA construct to monitor cAMP with high spatiotemporal resolution (DiPilato et al., 2004; Nikolaev et al., 2004; Nikolaev et al., 2006; Ponsioen et al., 2004; Zaccolo and Pozzan, 2002). Highly notable is a transgenic mouse model with ubiquitous expression of the cytosolic cAMP indicator Epac1-cAMP-sensor (Epac1-camps) (Calebiro et al., 2009). The short name of exchange factor directly activated by cAMP 1 is EPAC1, which is widely distributed in CMs. Epac1-camps contains a cAMP binding domain derived from Epac1, sandwiched between CFP and YFP as a FRET pair (Figure 10). In CMs, transient expression of such biosensors is commonly achieved via transfection of neonatal CMs or adenoviral gene transfer in adult CMs (Kirshenbaum et al., 1993). Expression of a functional FRET biosensor via adenovirus in adult myocytes requires at least 40-48 hours during which dedifferentiation and remodeling of normal cell physiology are likely to occur (Horackova and Byczko, 1997). 


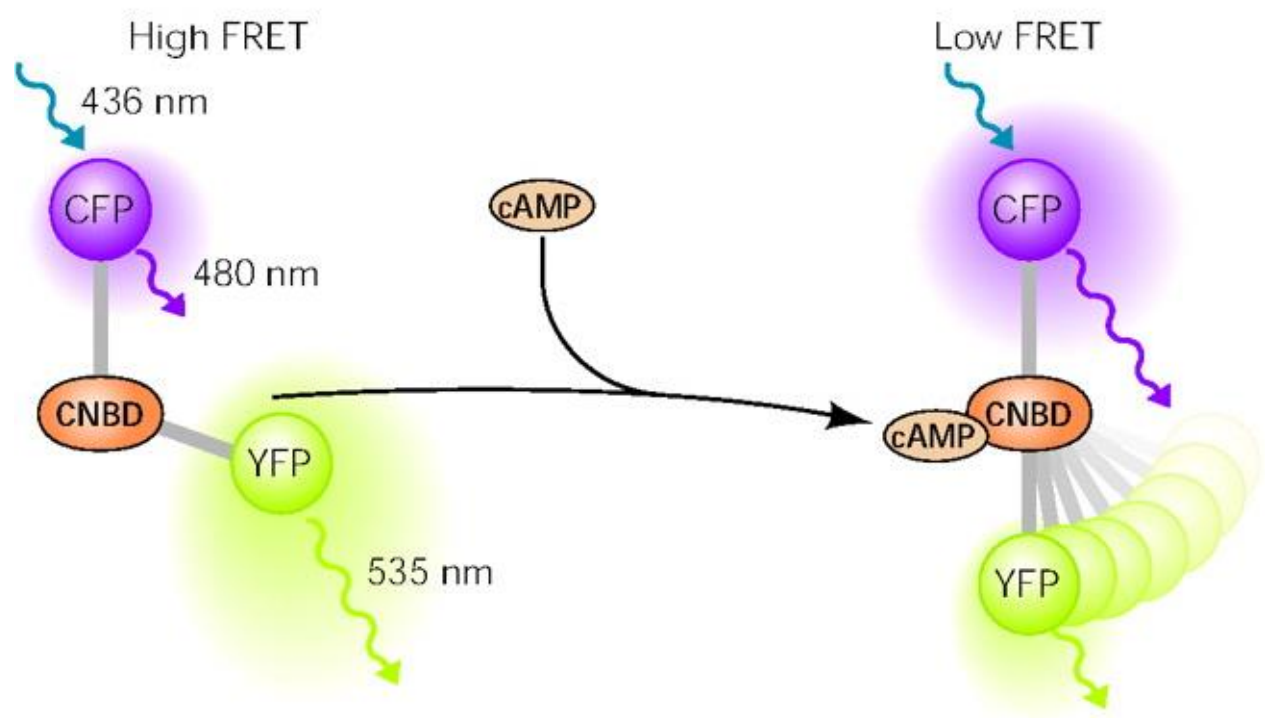

Figure 10. Principle of the Epac1-cAMP-sensor (Epac1-camps). Epac1-camps is based on a single cAMP binding domain from Epac1 fused between CFP and YFP. At low cAMP levels, the donor (CFP) and acceptor (YFP) are nearby and FRET occurs. Binding of cAMP to the cAMP binding domain induces a conformational change and a concomitant decrease in FRET. Excitation of CFP is at $436 \mathrm{~nm}, \mathrm{CFP}$ and YFP emission are collected at 480 and $535 \mathrm{~nm}$, respectively (Nikolaev and Lohse, 2006). With permission from American Physiological Society.

In CMs, several cAMP microdomains have been proposed, which are located, for example, around $\mathrm{Ca}^{2+}$ handling proteins such as LTCCs, RYR2 and sarco/endoplasmic reticulum $\mathrm{Ca}^{2+}$ ATPase 2a, since cAMP is not uniformly distributed within the cell but is rather organized in subcellular compartments (Fischmeister et al., 2006).

\subsection{Aims of this Ph.D. thesis}

The hypotheses of this project included: the CPVT phenotypes and pathophysiological mechanisms are mutation-specific; antiarrhythmic effects of drugs are mutation-specific. To test the hypotheses, the aims of this project were to establish in vitro disease models using iPSCs from six patients with CPVT carrying the different RYR2 heterozygous missense mutations (R420W, A2254V, E4076K, and H4742Y) in the domain a, b, c and d, to investigate the pathophysiological phenotypes of iPSC-CMs, to better understand the mutation-specific 
pathophysiological mechanism of the disease and to explore drug therapies. The scientific and technological main objectives of the project included:

(1) Generation and characterization of iPSC lines from two patients with CPVT carrying the mutation RYR2 $2^{\mathrm{R} 420 \mathrm{~W}}$ and $\mathrm{PSC}$ lines from one patient with CPVT carrying the mutation $\mathrm{RYR} 2^{\mathrm{H} 4742 \mathrm{Y}}$, as well as differentiation and characterization of iPSC-CMs.

(2) Electrophysiological phenotype characterization of CPVT-iPSC-CMs compared to CMs derived from iPSCs from healthy donors (Ctrl-iPSC-CMs).

(3) Test the efficacy of four antiarrhythmic drugs (flecainide, dantrolene, rycal1, rycal2) on CPVT-iPSC-CMs.

(4) Explore cAMP dynamic in CPVT-iPSC-CMs compared to Ctrl-iPSC-CMs. 


\section{Materials and methods}

\subsection{Materials}

\subsubsection{Cells}

Mouse embryonic fibroblasts (MEFs): isolated from 15- to 17-day-old embryos of Naval Medical Research Institute mice (Central Animal Facility, University Medical Center Göttingen).

Human induced pluripotent stem cells (hiPSCs): All hiPSC lines used in this study were generated in the laboratory of Prof. Guan and are listed in Table 2. The iPSC lines of WT-D2, iBM76.1 and iBM76.3 were generated previously from healthy donors using the lentivirus system containing the STEMCCA cassette (Streckfuss-Bomeke et al., 2013). The iPSC lines from CPVT patients b1, c1 and c2 were generated using both the STEMCCA lentivirus system and Sendai virus system. In this thesis work, the generation and characterization of the cell lines of CPVTa2, CPVTa3, and CPVTd1 patients were performed by using the Sendai virus system (Materials and methods 2.1.3). The usage of human tissue was approved by the ethics committee of Universitätsmedizin Göttingen (Az. 21/1/11).

Table 2. iPSC lines used in this study

\begin{tabular}{|c|c|c|c|c|c|}
\hline & Donors & \multirow{2}{*}{$\begin{array}{l}\text { a.a. } \\
\text { changes } \\
\text { R420W }\end{array}$} & \multirow{2}{*}{$\begin{array}{l}\begin{array}{l}\text { Somatic } \\
\text { cells }\end{array} \\
\text { CPVTa2 } \\
\text { CPVTa3 }\end{array}$} & \multirow{2}{*}{$\begin{array}{l}\text { Virus used } \\
\text { Sendai }\end{array}$} & \multirow{2}{*}{$\begin{array}{l}\text { iPSC lines per } \\
\text { patient } \\
\text { isCPVTa2.1-3 } \\
\text { isCPVTa3.1-3 }\end{array}$} \\
\hline CPVT & $\mathrm{a}$ & & & & \\
\hline & \multirow[t]{2}{*}{$\mathrm{b}$} & \multirow[t]{2}{*}{ A2254V } & \multirow[t]{2}{*}{ CPVTb1 } & STEMCCA & iCPVTb1.1-2 \\
\hline & & & & Sendai & isCPVTb1.2-3 \\
\hline & \multirow[t]{2}{*}{$\mathrm{c}$} & \multirow[t]{2}{*}{ E4076K } & \multirow[t]{2}{*}{$\begin{array}{l}\text { CPVTc1 } \\
\text { CPVTc2 }\end{array}$} & STEMCCA & $\begin{array}{l}\text { iCPVTc1.1, } 6 \\
\text { iCPVTc2.1-2 }\end{array}$ \\
\hline & & & & Sendai & $\begin{array}{l}\text { isCPVTc1.1, } \\
\text { isCPVTc2.2, } 4\end{array}$ \\
\hline & $\mathrm{d}$ & $\mathrm{H} 4742 \mathrm{Y}$ & CPVTd1 1 & Sendai & isCPVTd1.4, 10, 11 \\
\hline Control & 1 & No & $\mathrm{BM}$ & STEMCCA & iBM76.1, 3 \\
\hline
\end{tabular}




\begin{tabular}{|l|l|l|l|l|l|}
\hline & 2 & No & WT-D2 & STEMCCA & iWT-D2 \\
\hline
\end{tabular}

\subsubsection{Oligonucleotides}

All oligonucleotides used for polymerase chain reaction (PCR) were purchased from Eurofins MWG Operon. The sequences are listed in alphabetical order (Table 3) together with the amplified fragment length (F), annealing temperature (TA), and number of cycles (C).

Table 3. Oligonucleotides used for DNA sequencing and PCR analyses

\begin{tabular}{|c|c|c|c|c|}
\hline Gene & Sequence & $\mathrm{F}[\mathrm{bp}]$ & $\mathrm{TA}\left[{ }^{\circ} \mathrm{C}\right]$ & $\mathrm{C}$ \\
\hline$\overline{A F P}$ & $\begin{array}{l}\text { for: 5'-ACT CCA GTA AAC CCT GGT GTT G-3' } \\
\text { rev: 5'-GAA ATC TGC AAT GAC AGC CTC A-3' }\end{array}$ & 255 & 60 & 33 \\
\hline$A L B$ & $\begin{array}{l}\text { for: 5'-CCT TTG GCA CAA TGA AGT GGG TAA CC- } \\
3^{\prime} \\
\text { rev: } 5^{\prime}-\text { CAG CAG TCA GCC ATT TCA CCA TAG G-3' }\end{array}$ & 284 & 62 & 35 \\
\hline$\alpha-M H C$ & $\begin{array}{l}\text { for: 5'-GTC ATT GCT GAA ACC GAG AAT G-3' } \\
\text { rev: 5'-GCA AAG TAC TGG ATG ACA CGC T-3' }\end{array}$ & 413 & 60 & 35 \\
\hline cTNT & $\begin{array}{l}\text { for: 5'-GAC AGA GCG GAA AAG TGG GA-3' } \\
\text { rev: 5'-TGA AGG AGG CCA GGC TCT AT-3' }\end{array}$ & 305 & 55 & 35 \\
\hline $\begin{array}{l}R Y R 2 \\
\text { (CPVTa) }\end{array}$ & $\begin{array}{l}\text { for: 5'-GACGTTGGGAGTAATGGCCT-3' } \\
\text { rev: 5'- CGTTTCTGCTGACTTACCAAACAG-3' }\end{array}$ & 293 & 54 & 30 \\
\hline $\begin{array}{l}\text { RYR2 } \\
\text { (CPVTd) }\end{array}$ & $\begin{array}{l}\text { for: 5'-CAATAGTCATGCGTTCTGTTTTCTG-3' } \\
\text { rev: 5'-GAGACCCTCAAACTACTAATCCCT-3's }\end{array}$ & 370 & 55 & 30 \\
\hline FOXD3 & $\begin{array}{l}\text { for: 5'-GTG AAG CCG CCT TAC TCG TAC-3' } \\
\text { rev: 5'-CCG AAG CTC TGC ATC ATG AG-3' }\end{array}$ & 353 & 61 & 38 \\
\hline GAPDH & $\begin{array}{l}\text { for: 5'-AGA GGC AGG GAT GAT GTT CT-3' } \\
\text { rev: 5'-TCT GCT GAT GCC CCC ATG TT-3' }\end{array}$ & 265 & 55 & 34 \\
\hline GDF3 & $\begin{array}{l}\text { for: 5'-TTC GCT TTC TCC CAG ACC AAG GTT TC-3' } \\
\text { rev: 5'-TAC ATC CAG CAG GTT GAA GTG AAC } \\
\text { AGC ACC-3' }\end{array}$ & 331 & 54 & 32 \\
\hline LIN28 & $\begin{array}{l}\text { for: 5'-AGT AAG CTG CAC ATG GAA GG-3' } \\
\text { rev: 5'-ATT GTG GCT CAA TTC TGT GC-3' }\end{array}$ & 410 & 52 & 36 \\
\hline$N A N O G$ & $\begin{array}{l}\text { for: 5'-AGT CCC AAA GGC AAA CAA CCC ACT TC- } \\
3^{\prime}\end{array}$ & 164 & 64 & 36 \\
\hline
\end{tabular}




\begin{tabular}{|l|l|l|l|l|}
\hline & $\begin{array}{l}\text { rev: 5'-ATC TGC TGG AGG CTG AGG TAT TTC TGT } \\
\text { CTC-3' }\end{array}$ & & & \\
\hline SYP & $\begin{array}{l}\text { for: 5'-GCC TGT CTC CTT GAA CAC GAA C-3' } \\
\text { rev: 5'-TAC CGA GAG AAC AAC AAA GGG C-3' }\end{array}$ & 288 & 56 & 35 \\
\hline$T H$ & $\begin{array}{l}\text { for: 5'-GCG GTT CAT TGG GCG CAG G-3' } \\
\text { rev: 5'-CAA ACA CCT TCA CAG CTC G-3' }\end{array}$ & 215 & 60 & 34 \\
\hline
\end{tabular}

$\boldsymbol{A F P}$ : alpha-1-fetoprotein, $\boldsymbol{A L B}$ : albumin, $\boldsymbol{\alpha}-\boldsymbol{M H C}$ : myosin heavy chain (alpha), $\boldsymbol{c T N T}$ : cardiac troponin T, RYR2; Ryanodine receptor 2, FOXD3: forkhead box D3, GAPDH: glyceraldehyde3-phosphate dehydrogenase, $\boldsymbol{G D F 3}$ : growth differentiation factor 3, $\boldsymbol{T H}$ : tyrosine hydroxylase

\subsubsection{Sendai virus system used for cell reprogramming}

Sendai virus (called Hemagglutinating Virus of Japan) is a respiratory virus of mouse and rat, and is classified as mouse parainfluenza virus type I belonging to the Paramyxoviridae family. Sendai virus is an enveloped virus of $150-250 \mathrm{~nm}$ in diameter, whose genome is a singlestranded negative-sense RNA (15,384 bases). The Sendai virus system CytoTune®-iPS Reprogramming Kit was purchased from Thermo Fisher Scientific. The reprogramming vectors used in this study include the four Yamanaka factors OCT4, SOX2, KLF4, and c-MYC, shown to be sufficient for efficient reprogramming.

\subsubsection{Antibodies}

Table 4. Primary antibodies used for immunofluorescence

\begin{tabular}{|l|l|l|l|}
\hline Antigen & Type & Supplier & Dilution \\
\hline AFP & Polyclonal rabbit IgG & Dako, A0008 & $1: 100$ \\
\hline$\alpha$-actinin & mouse IgG1 & $\begin{array}{l}\text { Sigma-Aldrich } \\
\text { A7811 }\end{array}$ & $1: 1000$ \\
\hline$\beta$ III -tubulin & mouse IgG2A & $\begin{array}{l}\text { Covance }^{\circledR}, \text { MMS- } \\
\text { 435P }\end{array}$ & $1: 2000$ \\
\hline cTNT & mouse IgG1 & $\begin{array}{l}\text { Thermo Fisher } \\
\text { Scientific } \\
\text { MS295PABX }\end{array}$ & $1: 200$ \\
\hline Cx43 & rabbit IgG & Abcam ${ }^{\circledR}$, ab11370 & $1: 1000$ \\
\hline LIN28 & goat IgG & R\&D Systems $^{\circledR}$, & $1: 300$ \\
\hline
\end{tabular}




\begin{tabular}{|c|c|c|c|}
\hline & & AF3757 & \\
\hline MLC2a & mouse IgG2B & $\begin{array}{l}\text { Synaptic Systems, } \\
\text { 311-011 }\end{array}$ & $1: 500$ \\
\hline MLC2v & rabbit IgG & $\begin{array}{l}\text { Protein tech }{ }^{\circledR} 10906- \\
\text { 1-AP }\end{array}$ & $1: 200$ \\
\hline NANOG & goat $\operatorname{IgG}$ & $\begin{array}{l}\text { R\&D Systems }{ }^{\circledR}, \\
\text { AF1997 }\end{array}$ & $1: 200$ \\
\hline OCT4 & goat $\operatorname{IgG}$ & $\begin{array}{l}\text { R\&D Systems }{ }^{\circledR}, \\
\text { AF1759 }\end{array}$ & $1: 40$ \\
\hline Phalloidin & Alexa Fluor $^{\circledR} 555$ & $\begin{array}{l}\text { Life Technologies }{ }^{\mathrm{TM}} \text {, } \\
\text { A-12379 }\end{array}$ & $1: 100$ \\
\hline SMA & mouse IgG2A & $\begin{array}{l}\text { Sigma-Aldrich }{ }^{\circledR} \\
\text { A2547 }\end{array}$ & $1: 3000$ \\
\hline SOX2 & mouse $\operatorname{IgG} 2 \mathrm{~A}$ & $\begin{array}{l}\text { R\&D Systems }{ }^{\circledR} \\
\text { MAB2018 }\end{array}$ & $1: 50$ \\
\hline SSEA4 & mouse IgG & Abcam $^{\circledR}$, ab16287 & $1: 100$ \\
\hline TRA-1-60 & mouse IgM & Abcam $^{\circledR}$, ab16288 & $1: 200$ \\
\hline
\end{tabular}

$\boldsymbol{\alpha}$-actinin; alpha-actinin, $\boldsymbol{\beta}$ III -tubulin; Class III $\beta$-tubulin, Cx43; Connexin 43, MLC2a; myosin light chain 2 atrial, MLC2v; myosin light chain 2 ventricular, SMA; smooth muscle actin, SOX2; sex-determining region Y-Box 2, SSEA4; stage-specific embryonic antigen-4, TRA-1-60; tumour-related antigen-1-60

Table 5. Secondary antibodies

\begin{tabular}{|l|l|l|}
\hline Antibody & Supplier & Dilution \\
\hline $\begin{array}{l}\text { Alexa Flour 488-conjugated goat- } \alpha- \\
\text { mouse IgG }\end{array}$ & Life Technologies ${ }^{\mathrm{TM}}$, A-11001 & $1: 200$ \\
\hline Cy3-conjugated donkey- $\alpha$-goat IgG & Jackson ImmunoResearch, 705-166-147 & $1: 600$ \\
\hline $\begin{array}{l}\text { Cy3-conjugated goat- } \alpha \text {-mouse IgG + } \\
\text { IgM }\end{array}$ & Jackson ImmunoResearch, 115-165-068 & $1: 300$ \\
\hline Cy3-conjugated goat- $\alpha$-rabbit IgG & Jackson ImmunoResearch, 111-165-003 & $1: 800$ \\
\hline FITC-conjugated donkey- $\alpha$-rabbit IgG & Jackson ImmunoResearch, 711-095-152 & $1: 200$ \\
\hline FITC-conjugated goat- $\alpha$-mouse IgG & Jackson ImmunoResearch, 115-096-072 & $1: 200$ \\
\hline FITC-conjugated goat- $\alpha$-mouse IgM & Jackson ImmunoResearch, 115-095-020 & $1: 100$ \\
\hline
\end{tabular}




\subsubsection{Media, solutions, and chemicals used for cell culture}

Table 6. Components

\begin{tabular}{|c|c|}
\hline Components & Supplier \\
\hline Accutase $^{\mathrm{TM}}$ & PAA Laboratories, \#L11-007 \\
\hline Albumin (human recombinant) & Sigma-Aldrich $^{\circledR}, \#$ A0237 \\
\hline B-27 ${ }^{\circledR}$ serum-free supplement (50x) & Life Technologies ${ }^{\mathrm{TM}}$, \#17504044 \\
\hline B-27 $7^{\circledR}$ serum-free supplement (w/o insulin, 50x) & Life Technologies ${ }^{\mathrm{TM}}$, \#0050129SA \\
\hline$\beta$-mercaptoethanol & Serva Electrophoresis, \#28625 \\
\hline Bovine albumin fraction V solution (BSA, $7.5 \%$ ) & Life Technologies $^{\mathrm{TM}}$, \#15260037 \\
\hline CHIR99021 & Merck Millipore, \#361559 \\
\hline Collagenase type 2 & Worthington $^{\circledR}, \#$ LS004176 \\
\hline Collagenase type 4 & Worthington $^{\circledR}, \#$ LS004189 \\
\hline Dantrolene & Sigma-Aldrich $^{\circledR}, \#$ D9175 \\
\hline Dimethyl sulfoxide (DMSO) & Sigma-Aldrich $^{\circledR}, \#$ D2650 \\
\hline Dulbecco's modified Eagle medium (DMEM) & Life Technologies ${ }^{\mathrm{TM}}, \# 11960044$ \\
\hline DMEM/F-12 & Life Technologies ${ }^{\mathrm{TM}}, \# 31331028$ \\
\hline Dulbecco's phosphate buffered saline (DPBS) & Life Technologies ${ }^{\mathrm{TM}}$, \#14190094 \\
\hline Ethylenediaminetetraacetic acid (EDTA) & Sigma-Aldrich $^{\circledR}, \#$ E6758 \\
\hline Essential 8 8M $^{\mathrm{TM}}$ basal medium & Life Technologies ${ }^{\mathrm{TM}}$, \#A1516901 \\
\hline Essential $8^{\mathrm{TM}}$ Supplement & Life Technologies ${ }^{\mathrm{TM}}$,\#A1517101 \\
\hline Fetal bovine serum (FBS) & Sigma-Aldrich $^{\circledR}, \#$ F7524 / \\
\hline Fibroblast growth factor basic (bFGF) & PeproTech, \#100-18B \\
\hline Flecainide & Sigma-Aldrich $^{\circledR}, \# \mathrm{~F} 6777$ \\
\hline Gelatin & Sigma-Aldrich $^{\circledR}, \# 48720$ \\
\hline Geltrex ${ }^{\mathrm{TM}}$ & Life Technologies ${ }^{\mathrm{TM}}$, \#A1413301 \\
\hline HEPES sodium salt solution (1 M) & Sigma-Aldrich $^{\circledR}, \# \mathrm{H} 3662$ \\
\hline Iscove's modified Dulbecco's medium (IMDM) & Life Technologies ${ }^{\mathrm{TM}}, \# 31980022$ \\
\hline Knockout $^{\mathrm{TM}}$ serum replacement & Life Technologies ${ }^{\mathrm{TM}}$, \#10828028 \\
\hline L-Ascorbic Acid 2-Phosphate & Sigma-Aldrich®, \#A8960 \\
\hline L-glutamine $(200 \mathrm{mM})$ & Life Technologies ${ }^{\mathrm{TM}}, \# 25030024$ \\
\hline Mitomycin C & Serva Electrophoresis, \#29805.02 \\
\hline Monothioglycerol (MTG) & Sigma-Aldrich $^{\circledR}, \#$ M6145-25ML \\
\hline Non-essential amino acids (NEAA, 100x) & Life Technologies ${ }^{\mathrm{TM}}, \# 11140035$ \\
\hline Penicillin-streptomycin solution (100x) & Life Technologies ${ }^{\mathrm{TM}}$, \#15140-122 \\
\hline Pro-survival compound & Merck Millipore, \#529659 \\
\hline $\begin{array}{l}\text { Roswell Park Memorial Institute medium } 1640 \\
\text { (RPMI 1640) }\end{array}$ & Life Technologies ${ }^{\mathrm{TM}}$, \#72400-021 \\
\hline RPMI 1640 (w/o glucose) & Life Technologies ${ }^{\mathrm{TM}}$, \#11879-020 \\
\hline Sodium DL-lactate solution $60 \%(\mathrm{w} / \mathrm{w})$ & Sigma-Aldrich $^{\circledR}, \#$ L4263 \\
\hline Trypsin-EDTA $(0.25 \%)$, phenol red & Life Technologies ${ }^{\mathrm{TM}}$, \#25200056 \\
\hline Thiazovivin & Merck Millipore" \\
\hline Versene solution (0.48 mM EDTA) & Life Technologies ${ }^{\mathrm{TM}}$, \# 15040066 \\
\hline
\end{tabular}


Wnt antagonist II (IWP2)

Merck Millipore, \#681671

B27 medium: add one vial B-27® serum-free supplement (50x) into RPMI 1640, and stored at $4^{\circ} \mathrm{C}$ for up to 1 week

bFGF: dissolved in $0.1 \% \mathrm{v} / \mathrm{v}$ BSA/DPBS to a stock solution of $10 \mathrm{ng} / \mu \mathrm{l}$, aliquoted, and stored at $-20^{\circ} \mathrm{C}$

$\boldsymbol{\beta}$-mercaptoethanol (100x for cell culture): $7 \mu \mathrm{l}$ diluted in $10 \mathrm{ml}$ DPBS and sterile filtrated with Steriflip ${ }^{\circledR}$ filters $\left(0.22 \mu \mathrm{m}\right.$, Merck Millipore, \#SCGP00525), and stored at $4^{\circ} \mathrm{C}$

BSA/DPBS (1\% w/v): $1 \mathrm{ml}$ of $7.5 \% \mathrm{w} / \mathrm{v}$ BSA added to $6.5 \mathrm{ml}$ DPBS

Cardiac differentiation medium: $250 \mathrm{mg}$ Albumin (human recombinant) and $100 \mathrm{mg}$ Lascorbic acid 2-phosphate dissolved in $500 \mathrm{ml}$ RPMI 1640 with HEPES with GlutaMax and then filtered to sterilize (storage for up to 1 month at $4^{\circ} \mathrm{C}$ )

Cardiac digestion medium (100 $\mathbf{~ m l})$ : Cardiac differentiation medium $+20 \% \mathrm{FBS}+5-10 \mu \mathrm{M}$ Pro survival compound $+1 \mathrm{x}$ Pen/Strep (optional)

CHIR99021 (12 mM stock solution): $5 \mathrm{mg}$ dissolved in $0.894 \mathrm{ml} \mathrm{DMSO}$, aliquoted, and stored at $-20^{\circ} \mathrm{C}$

Collagenase type 2: dissolved in DMEM/F-12 to a working solution of $300 \mathrm{U} / \mathrm{ml}$, sterile filtrated, aliquoted and stored at $-20^{\circ} \mathrm{C}$

Collagenase type 4: dissolved in DMEM/F-12 to a working solution of $200 \mathrm{U} / \mathrm{ml}$, sterile filtrated, aliquoted and stored at $-20^{\circ} \mathrm{C}$

EDTA (50x): $1 \mathrm{~g}$ EDTA dissolved in $100 \mathrm{ml}$ DPBS; pH adjusted to 7.0 with $1 \mathrm{M} \mathrm{NaOH}$; sterile filtrated and stored at $4^{\circ} \mathrm{C}$

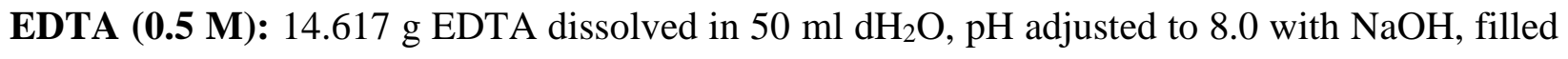
up to $100 \mathrm{ml}$ with $\mathrm{dH}_{2} \mathrm{O}$, sterile filtrated and stored at $-20^{\circ} \mathrm{C}$

EDTA dissociation solution: $500 \mu \mathrm{l}$ EDTA $(0.5 \mathrm{M})$ in $500 \mathrm{ml}$ DPBS, $0.9 \mathrm{~g} \mathrm{NaCl}$, adjust the osmolality to $340 \mathrm{mOsm}$; sterile filtrated and stored at $4^{\circ} \mathrm{C}$

Essential 8 8 $^{\mathrm{TM}}$ medium $(\mathbf{5 1 0} \mathbf{~ m l}): 500 \mathrm{ml}$ essential $8^{\mathrm{TM}}$ basal medium, $10 \mathrm{ml}$ essential $8^{\mathrm{TM}}$ 
Supplement

FBS: heat inactivated for $30 \mathrm{~min}$ at $56^{\circ} \mathrm{C}$

Feeder layer medium (100 ml): 84 ml DMEM, 15 ml FBS, 1 ml L-glutamine

Freezing medium (100 ml): $72 \mathrm{ml}$ DMEM, $20 \mathrm{ml} \mathrm{FBS,} 8 \mathrm{ml}$ DMSO

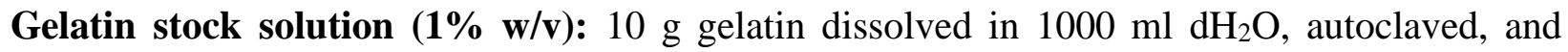
stored at $4{ }^{\circ} \mathrm{C}$

Geltrex $^{\text {TM: }} 2 \mathrm{mg}$ aliquoted and stored at $-80^{\circ} \mathrm{C}$; dissolved in $12 \mathrm{ml}$ cold DMEM/F-12 before use hESC medium (100 ml): $78 \mathrm{ml}$ DMEM/F-12, $20 \mathrm{ml} \mathrm{Knockout}^{\mathrm{TM}}$ serum replacement, $1 \mathrm{ml}$ NEAA (100x), 1 ml $\beta$-mercaptoethanol (100x), 10 ng/ml bFGF

Iscove medium (100 ml): 79 ml IMDM, 20 ml FBS, 1 ml NEAA, $450 \mu$ M MTG (freshly prepared)

IWP2 (5 mM stock solution): $10 \mathrm{mg}$ dissolved in $4.28 \mathrm{ml} \mathrm{DMSO}$ and incubated at $37^{\circ} \mathrm{C}$ for $10 \mathrm{~min}$, aliquoted, and stored at $-20^{\circ} \mathrm{C}$

Mitomycin C stock solution: dissolved in DPBS $(200 \mu \mathrm{g} / \mathrm{ml})$, aliquoted, and stored at $-20^{\circ} \mathrm{C}$

MTG (150 mM): $13 \mu 1$ MTG diluted in $1 \mathrm{ml} \mathrm{IMDM}$ and sterile filtrated with Millex ${ }^{\circledR}$-GS filter (0.22 $\mu \mathrm{m}$, Merck Millipore, \#SLGS033SS), freshly prepared before use

Pro-survival compound (5 mM stock solution): $10 \mathrm{mg}$ dissolved in $6.8 \mathrm{ml}$ DMSO, aliquoted, and stored at $-20^{\circ} \mathrm{C}$

Thiazovivin (2 mM): $10 \mathrm{mg}$ dissolved in $16.06 \mathrm{ml}$ DMSO, aliquoted, storage for up to 1 year at $-20^{\circ} \mathrm{C}$

2.1.6 Solutions, buffers, and chemicals for molecular biology, FRET and electrophysiological experiments

Table 7. Components for molecular biology, FRET and electrophysiological experiments

\begin{tabular}{|l|l|}
\hline Components & Supplier \\
\hline 3-isobutyl-1-methylxanthine (IBMX) & AppliChem, \#A0695.0001 \\
\hline
\end{tabular}




\begin{tabular}{|c|c|}
\hline Acetic acid & Sigma-Aldrich $^{\circledR}, \# 537020$ \\
\hline $\mathrm{Agar}^{\mathrm{TM}}$ & Serva Electrophoresis, \#200201 \\
\hline Alkaline phosphatase staining kit & Sigma-Aldrich $^{\circledR}, \# 86 \mathrm{R}-1 \mathrm{KT}$ \\
\hline AmpliTaq ${ }^{\circledR}$ DNA polymerase with buffer II & Life Technologies ${ }^{\mathrm{TM}}$, \#N808-0167 \\
\hline Bay $60-7550$ & Santa Cruz, \#SC-396772 \\
\hline Cilostamide & Sigma-Aldrich $^{\circledR}, \#$ C7971 \\
\hline $\begin{array}{l}\text { DAPI (4', 6-diamidino-2-phenylindole } \\
\text { dihydrochloride) }\end{array}$ & Sigma-Aldrich $^{\circledR}, \#$ D9542 \\
\hline DEPC-treated water & Ambion $^{\circledR}, \#$ AM9915G \\
\hline dNTP mix & Bioline, \#BIO-39029 \\
\hline Ethidium bromide & Roth $^{\circledR}, \# 2218$ \\
\hline Fluoromount-G ${ }^{\mathrm{TM}}$ & eBioscience, \#00-4958-02 \\
\hline Formalin (37\%) & Merck Millipore, \#1039991000 \\
\hline GeneRulerTM 100 bp Plus DNA Ladder & Thermo Fisher Scientific, \#0321 \\
\hline Giemsa stain & Sigma-Aldrich $^{\circledR}, \#$ GS500 \\
\hline GoTaq $^{\circledR}$ DNA polymerase & Promega, \#M3175 \\
\hline Isoproterenol & Sigma-Aldrich $^{\circledR}, \#$ I6504 \\
\hline $\begin{array}{l}\text { Karyomax }^{\circledR} \text { Colcemid }^{\circledR} \text { solution } \\
\text { (demecolcine) }\end{array}$ & Life Technologies $^{\mathrm{TM}}$, \#15210-040 \\
\hline Methanol & Sigma-Aldrich $^{\circledR}, \# 82762$ \\
\hline MuLV reverse transcriptase $(50 \mathrm{U} / \mu \mathrm{l})$ & Life Technologies ${ }^{\mathrm{TM}}$, \#N808-0018 \\
\hline NucleoSpin ${ }^{\circledR}$ Gel and PCR Clean-up & MACHEREY-NAGEL GmbH \& Co. \\
\hline Paraformaldehyde (PFA) & Sigma-Aldrich $^{\circledR}, \# 158127$ \\
\hline Polybrene $^{\circledR}$ & Sigma-Aldrich $^{\circledR}, \# 107689$ \\
\hline QIAquick $^{\circledR}$ gel extraction kit & Qiagen, \#28706 \\
\hline RNase inhibitor $(20 \mathrm{U} / \mu \mathrm{l})$ & Life Technologies ${ }^{\mathrm{TM}}$, \#N808-0119 \\
\hline Rolipram & Sigma-Aldrich $^{\circledR}, \#$ R6520 \\
\hline Rycal1 & From collaborator \\
\hline Rycal2 & From collaborator \\
\hline SV total RNA isolation system & Promega, \#Z3105 \\
\hline SYBR $^{\circledR}$ Green PCR master mix & Life Technologies $^{\mathrm{TM}}$,\#4309155 \\
\hline Triton X-100 & Sigma-Aldrich $^{\circledR}, \# 3051.3$ \\
\hline
\end{tabular}

0.1\% Triton $^{\mathrm{TM}} \mathbf{x}$-100/DPBS: $1 \mathrm{ml}$ Triton ${ }^{\mathrm{TM}} \mathrm{x}-100$ diluted in $9 \mathrm{ml}$ DPBS, storage at $4{ }^{\circ} \mathrm{C}$ for 6 months.

Agar gel (1.5\% or $2 \%$ w/v): $1.5 \mathrm{~g}$ or $2 \mathrm{~g}$ DNA Agar $^{\mathrm{TM}}$ dissolved in $100 \mathrm{ml} 1 \mathrm{x}$ TB buffer by boiling in a microwave, $6 \mu \mathrm{l}$ of $10 \mathrm{mg} / \mathrm{ml}$ ethidium bromide was added to $100 \mathrm{ml}$ agar gel at $65^{\circ} \mathrm{C}$ for visualization of DNA under ultraviolet light.

FRET buffer (in mM): $144 \mathrm{NaCl}, 5.4 \mathrm{KCl}, 1 \mathrm{MgCl}_{2}, 1 \mathrm{CaCl}_{2}, 10 \mathrm{HEPES}$, pH adjusted to 7.3 with $\mathrm{KOH}$. 


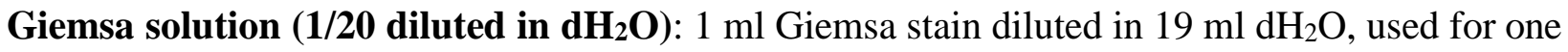
month.

KCl solution (0.075M): $5.59125 \mathrm{~g} \mathrm{KCl}$ dissolved in $1 \mathrm{~L} \mathrm{dH}_{2} \mathrm{O}$, used for one year.

PFA (4\% w/v): 4 g PFA dissolved in $100 \mathrm{ml}$ DPBS under heated conditions, used for 1 week.

TB buffer (5x): $108 \mathrm{~g}$ Tris, $55 \mathrm{~g}$ boric acid, filled up to $2000 \mathrm{ml}$ with $\mathrm{dH}_{2} \mathrm{O}$.

Pipette solution for action potential recording (in $\mathrm{mM}$ ):

(1): $122 \mathrm{~L}$-aspartic acid potassium salt, $10 \mathrm{NaCl}, 8 \mathrm{KCl}, 1 \mathrm{MgCl}_{2}, 5 \mathrm{Mg}$-ATP, $0.3 \mathrm{Li}-\mathrm{GTP}, 10$

HEPES; $\mathrm{pH}$ adjusted to 7.2 with $\mathrm{KOH}$.

(2): $60 \mathrm{KCl}, 100 \mathrm{~K}-G l u c o n a t e, 5$ Na2-Phosphocreatine, 4 Mg-ATP, $0.3 \mathrm{Na}-\mathrm{GTP}, 10 \mathrm{HEPES}$; pH adjusted to 7.2 with $\mathrm{KOH}$.

\subsection{Methods}

\subsubsection{Cell culture}

All cells were cultivated under humidified conditions at $37^{\circ} \mathrm{C}$ and $5 \%$ carbon dioxide (incubators from Thermo Fisher Scientific). All culture work was performed under sterile conditions using a laminar airflow cabinet (Thermo Fisher Scientific) to avoid microbiological contamination. Tissue culture dishes (35 x $10 \mathrm{~mm}, 60 \times 15 \mathrm{~mm}, 100 \times 20 \mathrm{~mm})$ and 6-, 12-well plates were bought from Starlab.

\subsubsection{Cultivation and inactivation of mouse embryonic fibroblasts}

MEFs were isolated from 15- to 17-day-old mouse embryos and subsequently cultivated on $0.1 \%$ gelatin-coated culture dishes in feeder layer medium for maximal four passages. Before used for cultivation of hiPSCs, MEFs were treated with a final concentration of $10 \mu \mathrm{g} / \mathrm{ml}$ mitomycin C for three hours at $37^{\circ} \mathrm{C}$ to stop cell proliferation. MEFs were washed three times with DPBS and subsequently treated with $0.25 \%$ trypsin-EDTA until the adherent cells started to detach. The 
single cells were resuspended in feeder layer medium and counted with a Thoma cell counting chamber. A certain number of cells (e.g. 3 x $10^{5}$ cells on 6 -cm dishes) were then plated onto $0.1 \%$ gelatin-coated culture dishes. The permit number is T10/6.

\subsubsection{Generation of hiPSCs}

Human iPSCs were generated by transducing human fibroblasts with the CytoTune ${ }^{\circledR}$-iPS Sendai Reprogramming Kit containing four Yamanaka transcription factors OCT3/4, SOX2, KLF4 and c-MYC. One day before transduction, human fibroblasts were plated onto $0.1 \%$ gelatin coated 12 -well plates with $0.3 \times 10^{5}$ cells per well. The cells were infected with the virus provided in the kit at a multiplicity of infection (MOI) of $0.5,1.0$ and 2.0 in freshly prepared $\mathrm{hESC}$ medium containing $1 \mu \mathrm{g} / \mathrm{ml}$ polybrene and $1 \mathrm{x}$ penicillin-streptomycin solution. On the next day, medium was changed with fresh hESC medium containing $1 \mathrm{x}$ penicillin/streptomycin solution after washing the cells with hESC medium twice. The medium was changed daily. On day 7, the cells were passaged and cultivated on inactivated MEFs. Single colonies that appeared with typical hESC morphology were picked mechanically using a sharpened glass pipette and were propagated further on MEFs.

\subsubsection{Cultivation of hiPSCs with feeder cells}

In some experiments, undifferentiated hiPSCs were cultivated on mitomycin $\mathrm{C}$ treated MEFs in hESC medium. The medium was changed daily, and colonies were split every 4 to 5 days. The cells were treated with $200 \mathrm{U} / \mathrm{ml}$ collagenase type 4 for $5 \mathrm{~min}$ at $37^{\circ} \mathrm{C}$ followed by two washing steps with DMEM/F-12. The cells were additionally cut into small clusters using a cell scraper (Sarstedt). After pipetting two to three times up and down, the cells were seeded onto new culture dishes with inactivated MEFs. Colonies with signs of differentiation were removed mechanically. 


\subsubsection{Feeder-free cultivation of hiPSCs}

In most experiments, hiPSCs were cultivated on culture dishes coated with growth factor reduced Geltrex $^{\mathrm{TM}}$ in feeder-free Essential $8^{\mathrm{TM}}$ medium. At about $85-90 \%$ confluency the cells were passaged onto new plates. The cells were washed two times and incubated for 3 to 4 min with EDTA dissociation solution or Versene solution at room temperature. The small cell clusters or single cells were subsequently transferred in Essential $8^{\mathrm{TM}}$ medium onto new culture dishes coated with Geltrex ${ }^{\mathrm{TM}}$. After passaging, pro-survival compound or thiazovivin (Xu et al., 2010) was added to the medium to a final concentration of $5 \mu \mathrm{M}$. The medium was changed daily.

\subsubsection{Spontaneous differentiation of hiPSCs in vitro}

Mass culture for spontaneous differentiation experiments started when iPSCs reached 90-100\% confluency. The cells were treated with $2.5 \mathrm{ml} 200 \mathrm{U} / \mathrm{ml}$ collagenase type 4 for 5 minutes at $37^{\circ} \mathrm{C}$. Cell colonies were cut with cell scraper into appropriate pieces. All colony pieces were collected with cell scraper from two culture plates and transferred to one medium added Petri dish. EBs were formed in suspension. The medium was changed to Iscove medium after 24 hours and every 2 days until day 8. For the medium change, cell suspensions were collected from all dishes to one $15 \mathrm{ml}$ tube, and then precipitated the cells by gravity (1-2 minutes). The supernatant was discarded and cell aggregates were transferred to new dishes. At day 8, about 50 EBs were prepared for pellet collection, and the rest were used for plating. 1x $6 \mathrm{~cm}$ dish was coated with $0.1 \%$ gelatin for pellet collection, $2 \times 6 \mathrm{~cm}$ dishes with 4 coverslips each were coated for immunostaining at $\mathrm{d} 8+5,8+25$. At day 8 , about $50 \mathrm{EBs}$ were prepared for pellet collection, and the rest were used for plating. The EBs were cultured in Iscove medium for another 25 days. The medium was changed every 2-3 days. Pellets were collected on $d 0, d 8, d 8+25$.

\subsubsection{Directed differentiation of hiPSCs into CMs and CM digestion and culture}

A protocol for directed cardiac differentiation was used to obtain a high number of CMs as described previously (Lian et al., 2012; Lian et al., 2013). At about 95-100\% confluency, the 
medium in undifferentiated hiPSCs cultivated under feeder-free conditions was replaced by cardiac differentiation medium supplemented with CHIR99021 at a final concentration of 4-10 $\mu \mathrm{M}$, a highly selective inhibitor of glycogen synthase kinase $3 \beta$. At day 2 (exactly $48 \mathrm{~h}$ later), the medium was carefully replaced by new cardiac differentiation medium supplemented with IWP2 at a final concentration of $5 \mu \mathrm{M}$. At day 6 , the medium was replaced with fresh cardiac differentiation medium without IWP2 and from day 8 on, the medium was replaced by B27 medium. First beating cells could be observed at day 7-8 of differentiation.

Around day 20, the more than $80 \%$ purify CMs were chosen for first digestion. Fresh collagenase 4 ( $200 \mathrm{U} / \mathrm{ml})$ diluted with B27 medium was added to the cells and incubated for 30 min to facilitate detachment of beating colonies from other cells. CM clusters after sedimentation for 5 minutes by gravity were treated with warmed $0.25 \%$ Trypsin-EDTA for $6-8 \mathrm{~min}$ at $37^{\circ} \mathrm{C}$. The clusters were carefully pipetted up and down to singularize cells. The cell suspension was transferred in $15 \mathrm{ml}$ Falcon tube with $2 \mathrm{ml}$ cardiac digestion medium containing 20\% FBS and 1x Pen/Strep, centrifuged for $5 \mathrm{~min}$ at 100-200 $\mathrm{g}$, filtered out the big clusters which were not digested yet by using the $40 \mu \mathrm{m}$ Falcon ${ }^{\mathrm{TM}}$ Cell Strainers, and resuspend in cardio digestion medium. The desired amount of cells was plated on Geltrex-coated 6-well plates (600000/well). After 60 days' routine medium change with B27 medium every 2-3 days for maturation-inducing, the differentiated CMs were digested again in the same way as described above and used for further experiments. The single CMs were plated onto $0.1 \%$ gelatin-coated $3-\mathrm{cm}$ tissue culture dishes and cultured further in the B27 medium for at least 14 days before used for AP measurements, and for at least 5 days before used for adenovirus infection for FRET experiments.

\subsubsection{Freezing and thawing of cultivated cells}

For cryopreservation, hiPSCs cultured on MEFs were treated with collagenase type 4 and dissected into small pieces as described before. hiPSCs cultured on Geltrex were treated with Versene Solution for 4-5 minute and dissociated into single cells and small clusters. The hiPSC clusters or single cells were transferred into $15 \mathrm{ml}$ reagent and centrifuge tubes (Sarstedt) and centrifuged at $200 \mathrm{~g}$ for $5 \mathrm{~min}$. After centrifugation, the supernatant was discarded and the cells were carefully resuspended in $1 \mathrm{ml}$ freezing medium and transferred into cryovials (Greiner Bio- 
one). The cells were kept overnight at $-80^{\circ} \mathrm{C}$ in a freezing box (Thermo Fisher Scientific) containing isopropanol to allow controlled freezing at $-1^{\circ} \mathrm{C}$ per min. The following day, frozen cells were transferred into liquid nitrogen.

For thawing, the frozen cells were placed in a $37^{\circ} \mathrm{C}$ warm water bath. After thawing, the cells were immediately diluted drop-wise in $10 \mathrm{ml}$ of cold DMEM/F-12 and centrifuged at $200 \mathrm{~g}$ for 5 min. The supernatant was discarded and the cell pellet was resuspended in hESC or Essential $8^{\mathrm{TM}}$ medium and plated onto 6-cm culture dishes either coated with inactivated MEFs or with Geltrex ${ }^{\mathrm{TM}}$, respectively.

\subsubsection{Alkaline phosphatase staining}

One of the first evidence for successful somatic cell reprogramming is alkaline phosphatase activity. In reprogrammed cells, alkaline phosphatase expression is significantly increased. Alkaline phosphatase activity in hiPSCs was detected using an alkaline phosphatase staining kit according to the manufacturer's instructions. Cells were washed with DPBS and subsequently fixed for $30 \mathrm{~s}$ using a fixation solution provided by the kit. Fixed cells were washed two times with distilled water and stained for 15 minutes at $37^{\circ} \mathrm{C}$ with a staining solution. After a final washing step with distilled water, cells were dried at room temperature. Alkaline phosphatase positive cells were stained in red.

\subsubsection{Genomic DNA isolation for DNA sequencing}

Feeder-free cultured iPSCs (without MEFs) were treated with TRlzol ${ }^{\circledR}$ reagent before genomic DNA isolation. The genomic DNA was isolated and purified using the $\mathrm{TRlzol}^{\circledR}$ reagent according to the manufacturer's instructions. The concentration of the isolated DNA was measured with a Nanodrop spectrophotometer at $260 \mathrm{~nm}$ and $280 \mathrm{~nm}$. For genomic DNA sequencing, the DNA sequence from the region of interest of $R Y R 2$ was initially amplified by PCR using the primer sets for CPVTa and CPVTd (Table 3). In each reaction, $50 \mathrm{ng}$ of total genomic DNA was used. The PCR product was electrophoretically separated on a $2 \%$ agar gel and subsequently excised with a scalpel. The DNA fragments were then extracted from the agar 
gel using the QIAquick ${ }^{\circledR}$ gel extraction kit according to the manufacturer's instructions using a refrigerated centrifuge. The DNA sequencing was performed by a commercial sequencing facility (Seqlab).

\subsubsection{Gene expression analyses}

\subsubsection{RNA isolation}

For gene expression analyses, cultured cells were washed with DPBS three times. Depending on the density, cells were lysed with 300-500 $\mu$ R RA lysis buffer. Total RNA isolation and purification was done with the SV total RNA isolation system according to the manufacturer's instructions. The concentration of the isolated RNA was measured with an Eppendorf BioPhotometer at $260 \mathrm{~nm}$ and $280 \mathrm{~nm}$. The RNA was subsequently used for reverse transcription (RT) reaction or stored at $-80^{\circ} \mathrm{C}$.

\subsubsection{Reverse transcription reaction}

After RNA isolation, total mRNA was transcribed into complementary DNA (cDNA) using the enzyme reverse transcriptase. All components for one RT reaction are listed in Table 8.

\section{Table 8. Components for reverse transcription reaction}

\begin{tabular}{|c|c|}
\hline Components for RT & $20 \mu$ l final volume \\
\hline 200 ng RNA + DEPC-treated $\mathrm{H}_{2} \mathrm{O}$ & $10.2 \mu \mathrm{l}$ \\
\hline 10x PCR buffer II & $2 \mu 1$ \\
\hline $25 \mathrm{mM} \mathrm{MgCl} 2$ & $4 \mu 1$ \\
\hline $100 \mathrm{nM}$ dNTPs & $0.8 \mu 1$ \\
\hline RNase inhibitor $(20 \mathrm{U} / \mu \mathrm{l})$ & $1 \mu \mathrm{l}$ \\
\hline $50 \mu \mathrm{M}$ Oligo d(T)16 & $1 \mu 1$ \\
\hline MuL V reverse transcriptase $(50 \mathrm{U} / \mu \mathrm{l})$ & $1 \mu \mathrm{l}$ \\
\hline
\end{tabular}

The RT reaction was accomplished in a thermocycler (SensoQuest) using the following program: $10 \min 22^{\circ} \mathrm{C}$ 


$\begin{array}{ll}50 \text { min } & 42^{\circ} \mathrm{C} \\ 10 \min & 95^{\circ} \mathrm{C} \\ \infty & 4{ }^{\circ} \mathrm{C}\end{array}$

The resulting cDNA was stored at $-80^{\circ} \mathrm{C}$.

\subsubsection{Polymerase chain reaction}

All components for one PCR to amplify certain cDNA fragments or templates are listed in Table 9.

Table 9: List of components for PCR.

\begin{tabular}{|l|l|}
\hline Components for PCR & $25 \mu \mathrm{l}$ final volume \\
\hline cDNA (template) & $1 \mu \mathrm{l}$ \\
\hline Nuclease-free $\mathrm{H}_{2} \mathrm{O}$ & $15.3 \mu \mathrm{l}$ \\
\hline $5 x$ Green GoTaq reaction buffer & $5 \mu \mathrm{l}$ \\
\hline Forward primer $(10 \mu \mathrm{M})$ & $1 \mu \mathrm{l}$ \\
\hline Reverse primer $(10 \mu \mathrm{M})$ & $1 \mu \mathrm{l}$ \\
\hline $10 \mathrm{mM}$ dNTPs & $1.6 \mu \mathrm{l}$ \\
\hline GoTaq G2 DNA polymerase $(5 \mathrm{U} / \mu \mathrm{l})$ & $0.1 \mu \mathrm{l}$ \\
\hline
\end{tabular}

The reaction was performed in a thermocycler using the following standardized program:

$95{ }^{\circ} \mathrm{C}$

$95^{\circ} \mathrm{C}$

$50-60{ }^{\circ} \mathrm{C}$

$72{ }^{\circ} \mathrm{C}$

$30 \mathrm{~s}$

$0 \mathrm{~s}$

$30 \mathrm{~s}\} 30-38$ cycles according to the primers used

$72{ }^{\circ} \mathrm{C}$

$30 \mathrm{~s}]$

$10 \min$

$4{ }^{\circ} \mathrm{C}$

$\infty$

\subsubsection{Gel electrophoresis}


All amplified PCR products were analyzed by electrophoretic separation on a $1.5 \%$ or $2 \%$ agar gel. The particular sizes of the DNA fragments were determined by GeneRuler ${ }^{\mathrm{TM}} 100 \mathrm{bp}$ Plus DNA Ladder. All results were documented under ultraviolet light with MultiImage Light Cabinet (Alpha Innotech Corporation).

\subsubsection{Immunofluorescence analyses}

Immunostainings were used to detect the expression and to show the localization of pluripotency-related proteins in undifferentiated hiPSCs, germ layer-specific proteins in differentiated hiPSCs, as well as cardiac-specific proteins in CMs. The hiPSCs and differentiated cells were initially cultivated on coverslips (Thermo Fisher Scientific). CMs were digested and the resulting single CMs were plated on 35-mm coverslips and further cultivated for at least 10 days before fixation. The cells were washed with DPBS two times, followed by fixation in $4 \%$ PFA at room temperature for 20 minutes with three further washing steps. The cells which were stained for nuclear transcription factors (OCT4, SOX2, and NANOG) as well as the cardiacspecific proteins cTNT, $\alpha$-actinin, Cx43 and MLC2a were additionally treated with $0.1 \%$ Triton $^{\mathrm{TM}} \mathrm{X}-100 / \mathrm{DPBS}$ for 10 minutes at room temperature. Before antibody incubation, the fixed cells were blocked in $1 \%$ BSA overnight at $4^{\circ} \mathrm{C}$. The cells were incubated with primary and subsequently with secondary antibodies, both diluted in $1 \%$ BSA for 1 hour at $37^{\circ} \mathrm{C}$ (NANOG at $4^{\circ} \mathrm{C}$ overnight). The nuclei were stained with $0.4 \mu \mathrm{g} / \mathrm{ml}$ DAPI. The stained cells were washed with $\mathrm{dH}_{2} \mathrm{O}$ once before mounting with Fluoromount- $\mathrm{G}^{\mathrm{TM}}$. Fluorescent images were taken with a fluorescence microscope (Zeiss Observer.Z1 or Axio Imager.M2).

\subsubsection{Teratoma formation and analysis}

The experiment was approved by the Lower Saxony State Office for Consumer Protection and Food Safety (Az. 33.14.42502-04-113/09). Undifferentiated hiPSCs were mechanically dissected using a cell scraper and transferred into 200-300 $\mu$ l DPBS. The cell clusters were injected subcutaneously into mice with deficiency in recombination activating gene 2 and gamma $\mathrm{C}$ (RAGC mice), which lack B cells, $\mathrm{T}$ cells, and natural killer cells. Teratomas were collected 
about three months after injection and fixed in phosphate buffered formalin ( $\mathrm{pH}$ 7.0) for 4 hours at room temperature or $4{ }^{\circ} \mathrm{C}$ overnight. After washing with $\mathrm{dH} 2 \mathrm{O}$, the teratomas were dehydrated and paraffinized using the Benchtop Tissue Processor 1020 (Leica Biosystems). The samples were embedded into paraffin using a tissue embedding system (Leica Biosystems) and processed into 6- $\mu \mathrm{m}$ sections with a microtome (Leica Biosystems). The histological sections were stained with hematoxylin and eosin (H\&E) at the Department of Pathology at the University Medical Center Göttingen and subsequently analyzed under a light microscope (Zeiss).

\subsubsection{Karyotyping}

All hiPSCs were cultured on Geltrex ${ }^{\mathrm{TM}}$ before analyzing the karyotypes. The cells were treated with $100 \mathrm{ng} / \mathrm{ml}$ of the microtubule-depolymerizing drug colcemid (Karyomax ${ }^{\circledR}$ Colcemid $^{\circledR}$ solution) for 16 hours. The supernatant containing detached cells was collected in a $15 \mathrm{ml}$ reagent and centrifuge tube and the adherent cells were washed with DMEM once and subsequently treated with TrypLE ${ }^{\mathrm{TM}}$ for 1 minute at $37^{\circ} \mathrm{C}$. Single cells were collected in the 15 $\mathrm{ml}$ tube and centrifuged at $200 \mathrm{~g}$ for $5 \mathrm{~min}$. The supernatant was discarded, leaving about $0.5 \mathrm{ml}$ in which the cell pellet was resuspended by tapping carefully against the tube. Pre-warmed $\left(37^{\circ} \mathrm{C}\right)$ $\mathrm{KCl}$ solution $(0.075 \mathrm{M})$ was added drop-wise to the cell suspension up to $8 \mathrm{ml}$ while shaking the tube carefully. After $45 \mathrm{~min}$ of incubation at $37^{\circ} \mathrm{C}$, the cells were centrifuged and the supernatant was aspirated to a residue of $0.5 \mathrm{ml}$, in which the cells were resuspended again. Freshly prepared and pre-cooled $\left(-20^{\circ} \mathrm{C}\right)$ methanol:acetic acid solution (1:3 freshly prepared) was then added drop-wise to the suspension while shaking the tube carefully. The cells were incubated on ice for 10 minutes and then centrifuged. The fixation step was repeated two times. After the third fixation, the cells were resuspended in a final volume of about $2 \mathrm{ml}$ to obtain an optimal density before dropped onto cold microscope slides. After air-drying, the chromosomes were stained with Giemsa solution $\left(1 / 20\right.$ diluted in $\left.\mathrm{dH}_{2} \mathrm{O}\right)$ for 5 minutes and washed twice with tap water. The stained chromosomes were counted under a light microscope (Zeiss Axio Imager, M2) and documented using the karyotyping analysis software Case Data Manager 6.0 (Applied Spectral Imaging, ASI). 


\subsubsection{Flow cytometry}

Single CMs were obtained by digestion with $0.25 \%$ trypsin-EDTA. Three tubes of cells were prepared, one tube as negative control without staining, the other two as sample and negative control stained only with the second antibody, respectively. Cells were fixed with $4 \%$ PFA solution. To permeate the cell membrane, $0.1 \%$ Triton X-100/1\% BSA was used to treat the cells for 15 minutes. The cells were incubated with the primary antibody (cTNT) overnight at $4^{\circ} \mathrm{C}$. Alexa flour 488 was used as the second antibody for 30-45 minute at room temperature in the dark. One tube of cells were stained with the second antibody alone. The setup of $\mathrm{BD}^{\mathrm{TM}}$ FACSAria and the software of $\mathrm{BD}^{\mathrm{TM}}$ FACSDiva 6.1 were used, following the instruments of technician.

\subsubsection{Action potential measurements and data analysis}

All patch-clamp recordings were performed at room temperature using an EPC10 USB patch clamp amplifier together with the PatchMaster software $\left(\mathrm{HEKA}^{\mathrm{TM}}\right)$. APs were measured with the ruptured-patch whole-cell current clamp technique. Spontaneous APs were recorded in RPMI 1640 basal medium immediately after rupturing with self-pulled microelectrodes with 3-5 M $\Omega$ resistance (thin-wall capillaries, WPI). No current was injected into the cells. In some experiments, CMs were paced with $0.2,0.4,0.8$, and $1.0 \mathrm{~Hz}$ for action potential duration (APD) analysis. The concentrations of four drugs recruited for analysis are: $1 \mu \mathrm{M}$ for flecainide, $1 \mu \mathrm{M}$ for dantrolene, $10 \mu \mathrm{M}$ for rycal1, and $10 \mu \mathrm{M}$ for rycal2. The CMs were kept in the incubator during the drug treatment for 1 to 2 hours. APs were analyzed using LabChart ${ }^{\circledR} 8$ Pro software (AD Instruments) to determine the beating frequency, resting membrane potential (RMP), the maximal upstroke velocity $\left(\mathrm{V}_{\max }\right)$, the AP amplitude (APA), and the APDs at $50 \%$ and $90 \%$ of repolarization (APD50 and APD90). The parameters of 5 to 10 APs in a row were averaged for the analysis. The putative DADs were defined as the amplitude of a depolarization in RMP stage is more than $5 \mathrm{mV}$. The EADs were defined as abnormal depolarization occurs during phase of repolarization. The rhythmic cells were defined as show rhythmic beating and do not appear DADs, EADs and TAs (DAD- or EAD-induced). The Number of DADs/TAs was calculated by 
$100 *$ number of DADs and DAD-induced TAs/beating frequency /recording time. The number of EADs and EAD-induced TAs was analyzed in the same way.

\subsubsection{Microelectrode array experiments and data analysis}

For the multielectrode array (MEA) measurements, the CMs with purity more than $80 \%$ were dissociated with $0.25 \%$ Trypsin-EDTA for $8 \mathrm{~min}$ at $37^{\circ} \mathrm{C}$, centrifuged for $5 \mathrm{~min}$ at $1,000 \mathrm{rpm}$ and resuspended in digestion medium. Then, 50,000 cells were plated in each well of a 6-well MEA (60-6well MEA200/30iR-Ti-tcr, Multi Channel Systems) coated with $21 \mu \mathrm{g} / \mathrm{cm}^{2}$ Geltrex in DMEM/F-12 medium. After $24 \mathrm{~h}$ and every second day, the medium was changed to the B27 medium. The cells on MEAs were cultured in B27 medium for 6 to 7 days before being used for recording.

Field potentials (FPs) were recorded at a sampling rate of $1 \mathrm{kHz}$ or $10 \mathrm{kHz}$ with the MC-Rack software at $37^{\circ} \mathrm{C}$ by using the TC02 temperature controller (Multi Channel Systems). Every MEA was firstly recorded for 3 minutes at basal condition. Then, $100 \mathrm{nM}$ ISO was added for 15 min and the same MEA was recorded for another 3 minutes. The last MEA recording has been done for another 3 minutes after 1-2-hour-treatment with one of the investigated drugs. The MEA was kept in the incubator during the drug treatment. The concentrations of four drugs recruited for analysis are the same as used in patch-clamp experiments $(1 \mu \mathrm{M}$ flecainide, $1 \mu \mathrm{M}$ dantrolene, $10 \mu \mathrm{M}$ rycal1 and $10 \mu \mathrm{M}$ rycal2). The frequency of FPs was determined by beats per minute from the last minute of the recording of each measurement. Based on recording results, ECG-like waveforms were analyzed for patterns similar to proarrhythmic events including PVC, VT. The arrhythmic cultures were defined as there are PVC-like events or VT-like events in the culture during 3 minutes recording time. The number of PVC-like events was quantified by the number of PVCs in every culture well divided by the recording time. Duration of VT-like events was normalized by dividing VT-like duration time to recording time.

\subsubsection{FRET measurements and data analysis}


To measure the cAMP dynamics with FRET biosensors, the sensors needed to be introduced into CMs. Adenoviruses carrying Epac1-camps and Epac1-camps-JNC were generated by the group of Prof. Nikolaev. CMs were plated onto coverslips at least 5 days before infection. The adenoviruses were mixed with B27 medium (MOI=10). CMs were incubated with the viruscontaining medium for 48 hours. Before starting FRET measurement, the cells were washed two times with RPMI 1640 medium to remove the virus. The cells were washed once with $300 \mu 1$ of FRET buffer (in mM: $144 \mathrm{NaCl}, 5.4 \mathrm{KCl}, 1 \mathrm{MgCl}_{2}, 1 \mathrm{CaCl}_{2}, 10 \mathrm{HEPES}, \mathrm{pH} 7.3$ ), and $300 \mu \mathrm{l}$ of the same buffer were added to the chamber. CMs were identified by the presence of contractions. CMs were initially stimulated with $1 \mathrm{nM}$ or $10 \mathrm{nM}$ ISO. For PDE2, PDE3 and PDE4 inhibition, $100 \mathrm{nM}$ Bay 60-7550, $10 \mu \mathrm{M}$ cilostamide and $10 \mu \mathrm{M}$ rolipram were used respectively. To inhibit all PDEs, $100 \mu \mathrm{M}$ IBMX was used. The measurements were performed with an inverted fluorescent microscope (Zeiss Axio Observer A1 or Olympus X71). Pictures were taken every 5 seconds with 30-100 ms exposure time. Signals for CFP and YFP were measured. Three hundred $\mu l$ of the desired compound solution (in FRET buffer) was added into the chamber as soon as the FRET ratio reached a stable baseline. FRET imaging data were analyzed offline using Origin 8 software. The CFP and YFP intensities were copy-pasted into an Excel or Origin 8 spreadsheet to calculate the corrected FRET ratio. The bleedthrough of the donor fluorescence (CFP) into the acceptor (YFP) channel was corrected, in this study, the bleedthrough ratio $=(\mathrm{YFP}-0.63 * \mathrm{CFP})$ / CFP for Zeiss Axio Observer system or ratio $=(\mathrm{YFP}-0.7 *$ CFP $) /$ CFP for Olympus X71 system.

\subsubsection{Statistical analysis}

Experimental data obtained from MEA, FRET and patch clamp analyses were presented as mean \pm standard error of mean (SEM). Arrhythmic cells and arrhythmic cultures were tested by using two-tailed Fisher's exact test by a $2 \times 2$ contingency table. For comparing variables in patchclamp and FRET experiments, the two-tailed unpaired student's $t$-test was applied. For comparing variables in MEA data, the two-tailed paired student's $t$-test was applied. All the $\mathrm{N}$ numbers were listed in the figure legends. Statistical significance was expressed by the p-value represented as $*(\mathrm{p}<0.05), * *(\mathrm{p}<0.01)$, and $* * * \mathrm{p}<0.001$ or as $\dagger(\mathrm{p}<0.05), \dagger \uparrow(\mathrm{p}<0.01)$, and 
$\dagger \dagger$ ( $\mathrm{p}<0.001)$. All statistical analyses were performed by using GraphPad Prism ${ }^{\circledR}$, Office Excel or Origin ${ }^{\circledR} 8.0$ software. 


\section{Results}

\subsection{Generation of hiPSCs and proof of pluripotency}

The isolated fibroblasts from the CPVT patients were transduced with Sendai virus particles between passage 2 and 4. All the reprogramming protocols were referenced to CytoTune ${ }^{\circledR}$-iPS Reprogramming Kit user manual. The first hiPSC colony-like areas appeared on day 11 after transduction. Several colonies resembling hiPSCs with respect to their morphology were picked mechanically and propagated further on new culture dishes coated with feeder cells. At least three independent cell lines for the CPVTa2 (isCPVTa2-1, -2, -3), CPVTa3 (isCPVTa3-1, -2, -3) and CPVTd1(isCPVTd1-4, -10, -11) patients were further characterized (Figure 11). The alkaline phosphatase was expressed in all generated hiPSC lines (Figure 11). Due to the heavy workload, this thesis is responsible for generation and characterization of isCPVTa2-1, $-2,-3$, isCPVTa3-1, -2, -3 and isCPVTd1-4, -10, -11 iPSC lines.

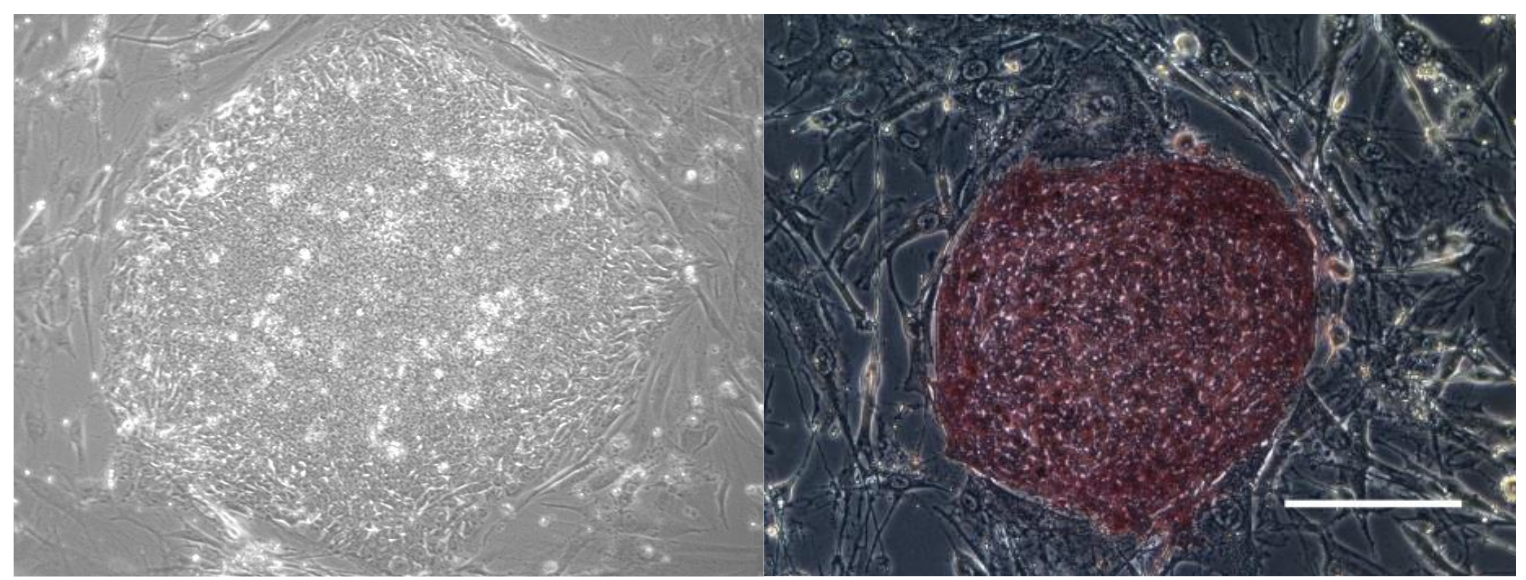

Figure 11 Morphology and alkaline phosphatase staining of generated hiPSCs. The respectively iPSC clones showed typical hiPSCs morphology and alkaline phosphatase activity. Scale bar: $200 \mu \mathrm{m}$.

\subsubsection{Expression of pluripotency-related markers}

Cellular reprogramming involves an extensive remodeling of gene expression. The expression patterns of the pluripotency-related marker genes SOX2, OCT4, NANOG, LIN28, GDF3, and 
FOXD3 were investigated by semiquantitative RT-PCR analyses in the generated isCPVTa2, isCPVTa3, and isCPVTd1 iPSC lines and compared to their original fibroblasts (Figure 12). Obvious upregulation of these genes in all generated hiPSC clones was observed.

A

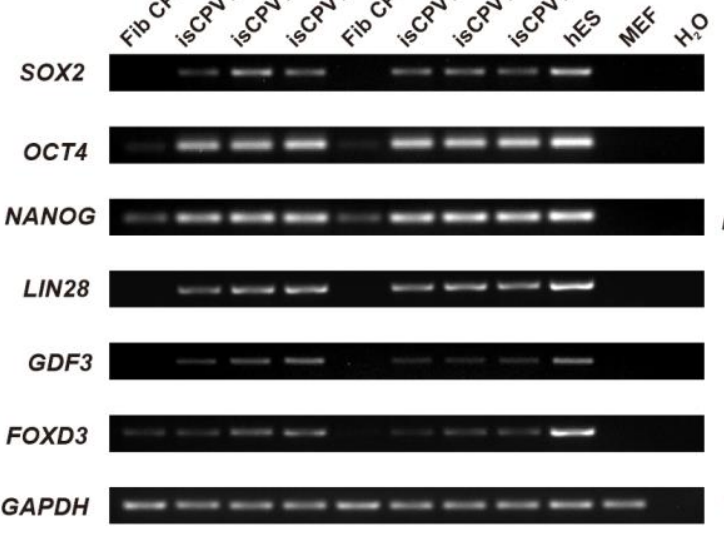

B

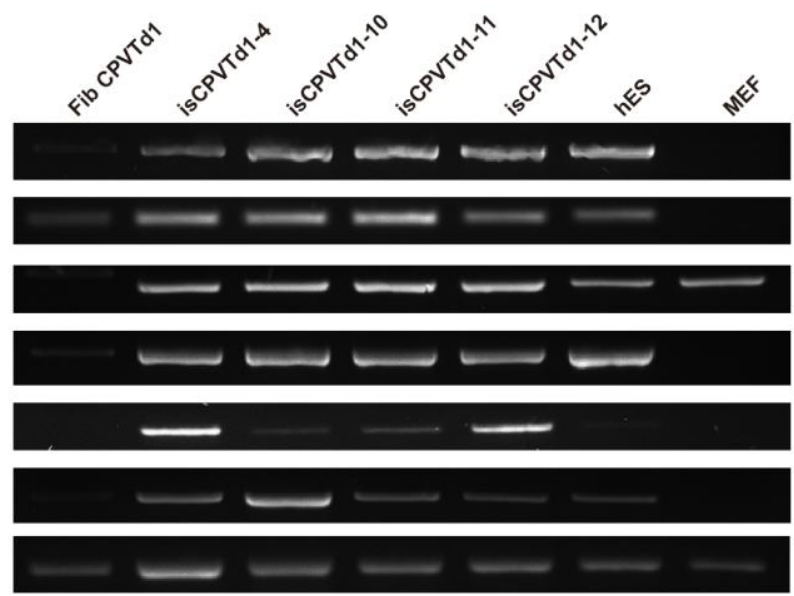

Figure 12. Gene expression analysis of generated hiPSCs. The pluripotency-related genes SOX2, OCT4, NANOG, LIN28, GDF3, and FOXD3 were highly expressed in human embryonic stem cells and in the generated isCPVTa2, isCPVTa3 (A) and isCPVTd1 iPSC lines (B). These genes were lower expressed in the original fibroblasts as well as in MEFs. hES: human ESCs; Fib CPVTa2, Fib CPVTa3 and Fib CPVTd1: fibroblasts from CPVT patients a2, a3 and d1.

The presence of selected pluripotency markers was confirmed by immunofluorescent staining of derived iPSC lines. In all analyzed hiPSC lines isCPVTa2-1, -2, -3, isCPVTa3-1, -2, -3 and isCPVTd1-4, -10, -11, transcription factors such as NANOG, OCT4, and SOX2 were proved to be present and located in the nuclei. LIN28 was located in the cytoplasm, whereas SSEA4 and TRA1-60 were observed on the membrane surface (Figure 13). 

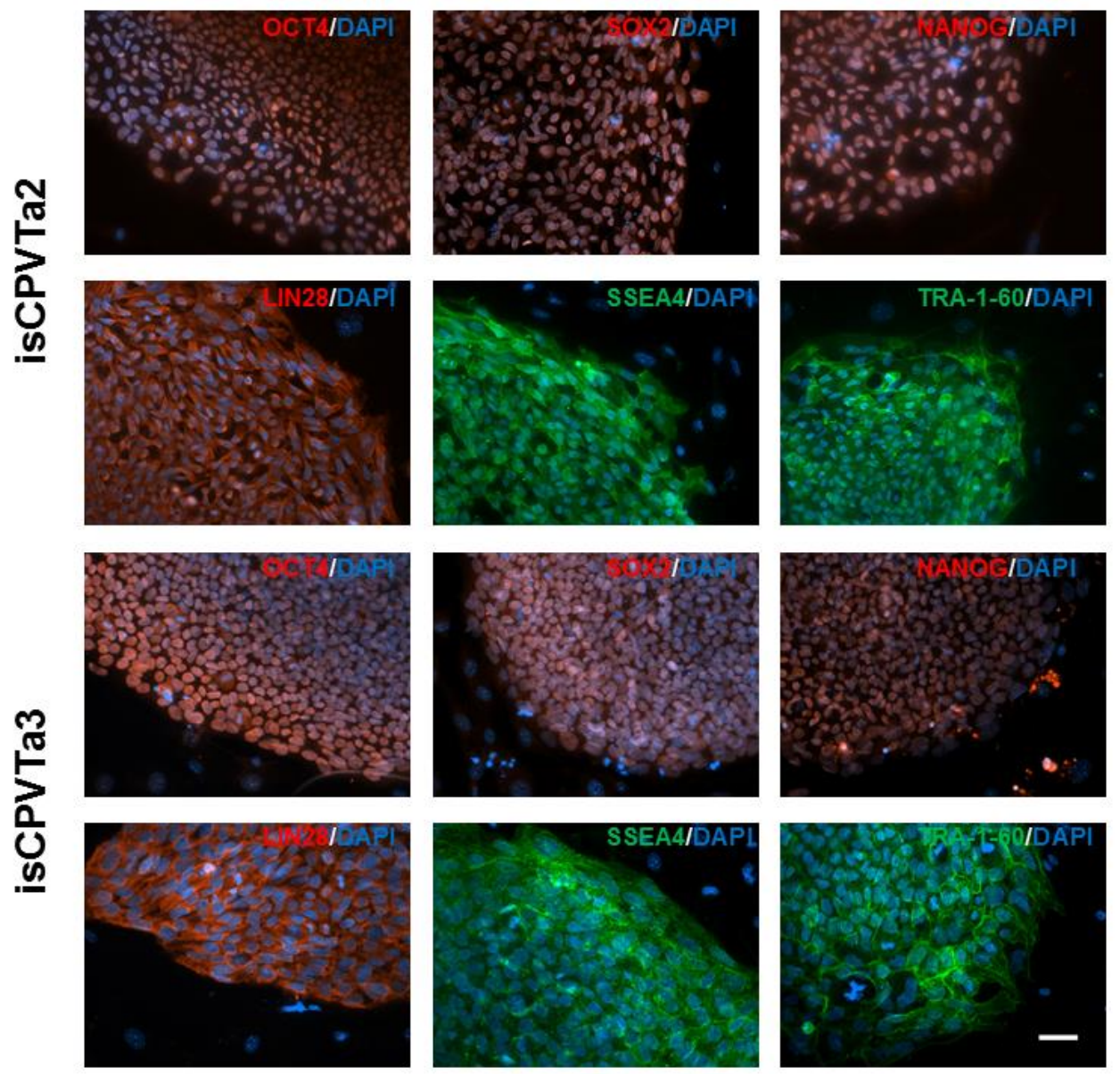

Figure 13. Immunostaining of pluripotency-related proteins in hiPSCs. All generated and analyzed iPSCs were positive for the transcription factors NANOG (in red), OCT4 (in red), and SOX2 (in red), as well as for the cytoplasmic located marker LIN28 (in red). SSEA4 (in green) and TRA-1-60 (in green), both located in the cell membrane were also detectable. The cell nuclei were stained with DAPI (in blue). Representative pictures from isCPVTa2-1, -2, -1, -1, -3, -1 for OCT4, SOX2, NANOG, LIN28, SEEA4, TRA1-60, respectively, and isCPVTa3-2, -2, -2, -1, -2, -3 were shown for OCT4, SOX2, NANOG, LIN28, SEEA4, TRA1-60, respectively. Scale bar: $50 \mu \mathrm{m}$.

\subsubsection{Differentiation potential in vitro and in vivo}

The differentiation potential of the generated hiPSCs in vitro and in vivo was determined by the spontaneous EB differentiation and teratoma formation, respectively. For the in vitro differentiation, the isCPVTa2, isCPVTa3, and isCPVTd1 cells were cultivated in suspension for 8 days in Iscove medium. The cells formed multicellular aggregates known as EBs during this

cultivation. The EBs were plated onto gelatin-coated culture dishes at day 8 for further differentiation. The mRNA was isolated and reverse transcribed into cDNA at three different 
time points (day 0 , day 8 , and day $8+25$ ). The endodermal marker gene $A F P$ and a late marker for hepatocytes, $A L B$, were expressed during differentiation. The mesodermal marker genes $c T N T$ and $\alpha-M H C$ were expressed during EB differentiation. Both ectodermal marker genes SYP and $T H$ were also expressed in each differentiated cell clone (Figure 14).
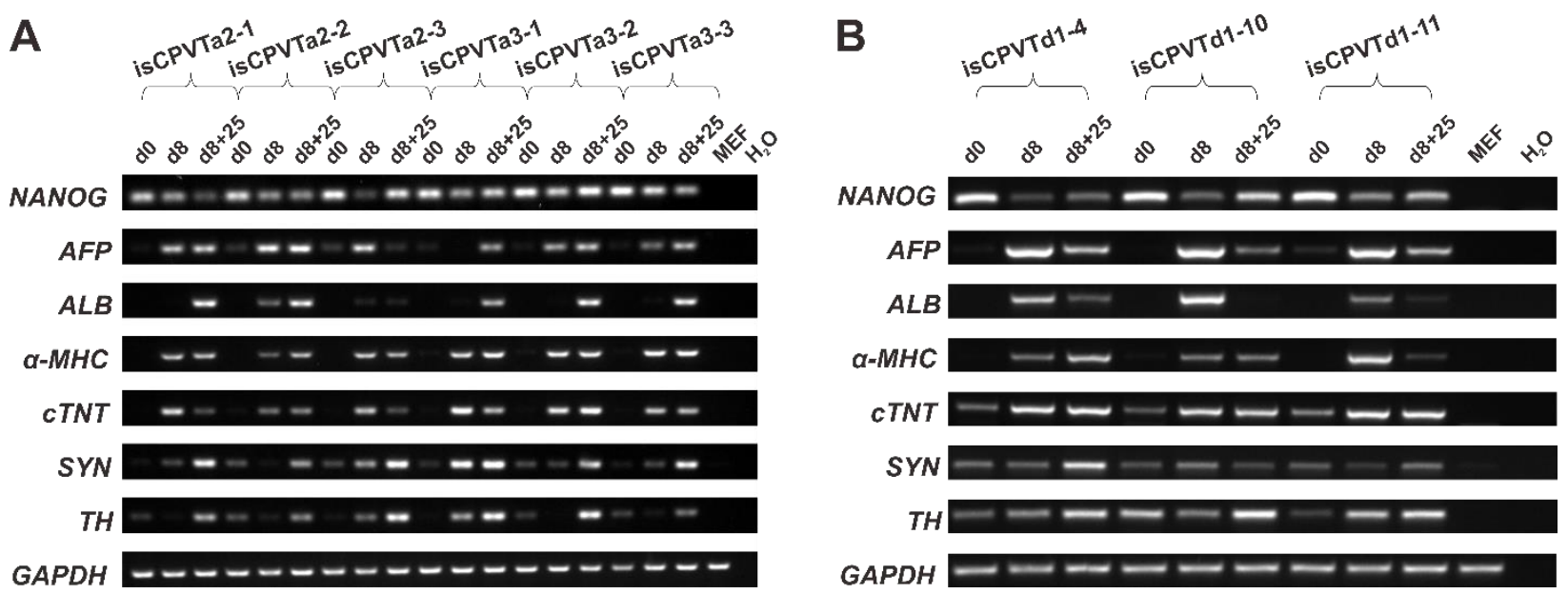

Figure 14. Gene expression analysis of spontaneously differentiated hiPSCs. The analyzed iPSC lines isCPVTa2-, isCPVTa3 (A) and isCPVTd1 (B) were differentiated spontaneously in vitro and mRNA was isolated on day $0(\mathrm{~d} 0)$, day $8(\mathrm{~d} 8)$, and day $8+25(\mathrm{~d} 8+25)$ of differentiation. Ectodermal $(A F P, A L B)$, mesodermal $(\alpha-M H C, c T N T)$ and endodermal $(S Y N, T H)$ marker genes were expressed during EB differentiation in a developmentally controlled manner. MEFs were used as negative control.

Furthermore, all the three germ layer protein markers: the endodermal marker AFP, the mesodermal marker SMA as well as the neuroectodermal marker class III $\beta$-tubulin were detected by immunofluorescence staining among the differentiated cells from all analyzed iPSC lines isCPVTa2, isCPVTa3, and isCPVTd1 (Figure 15). 

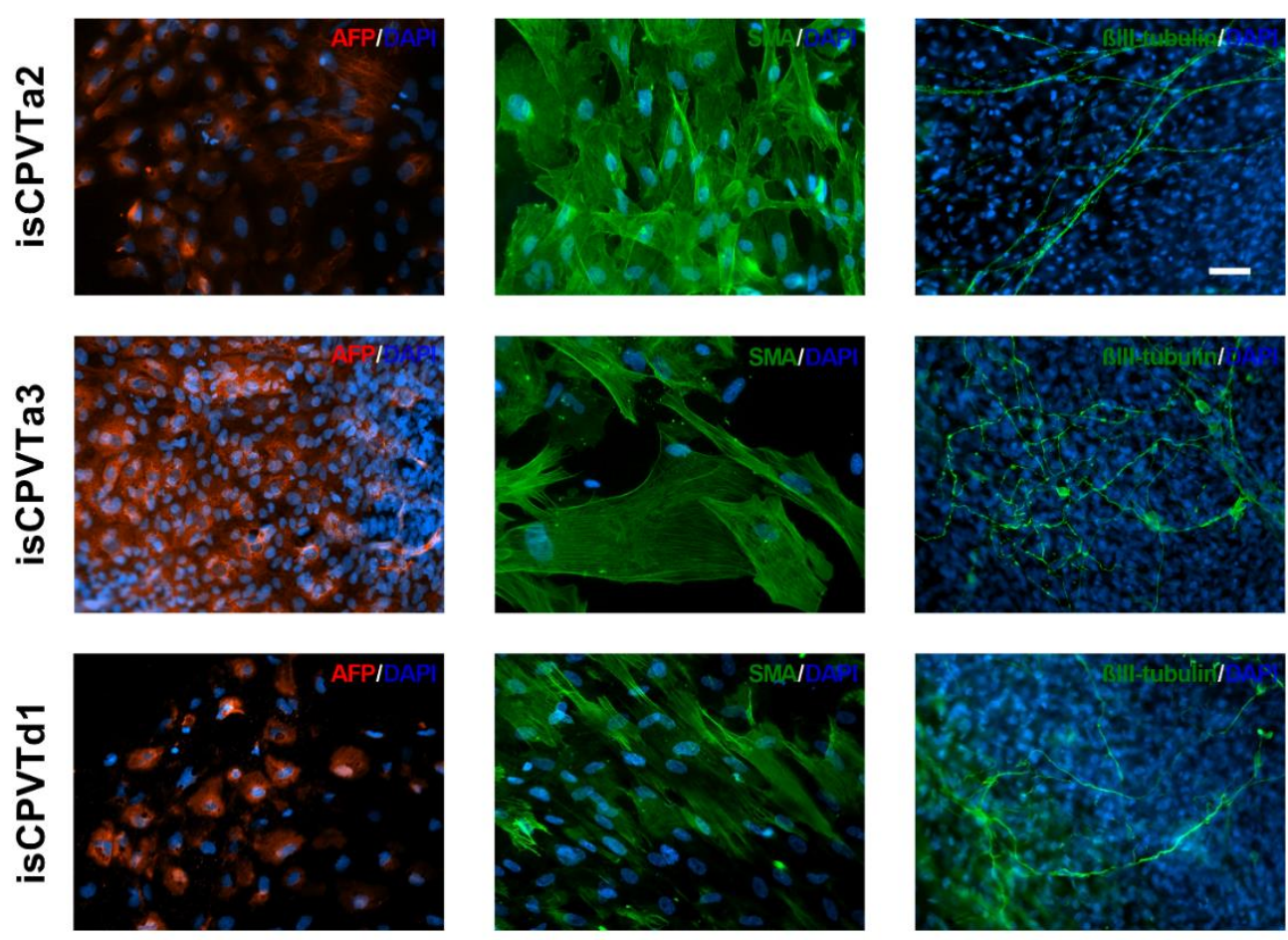

Figure 15. Immunostaining of cells during spontaneous differentiation of hiPSCs. After 33-day spontaneous differentiation, markers for the three germ layers were detectable in differentiating cells of isCPVTa2, isCPVTa3 and isCPVTd1. The respective markers for endodermal, mesodermal and ectodermal are $\alpha$-fetal protein (AFP), smooth muscle actinin (SMA) and class III $\beta$-tubulin. The cell nuclei were stained with DAPI (in blue). Scale bar: $50 \mu \mathrm{m}$.

Subcutaneous injection of hiPSCs into immunodeficient RAGC mice was used to test their differentiation potential in vivo. The injected cells formed mature teratomas, which were analyzed histologically (Figure 16). The teratomas derived from all analyzed iPSC lines isCPVTa2, isCPVTa3 and isCPVTd1 contained derivatives of all three embryonic germ layers, which showed intestinal tissue as endodermal tissues (Figure 16A, E, I), cartilage (Figure 16B, $\mathbf{F}, \mathbf{J}$ ) and muscle cells (Figure 16C, G, K) as mesodermal tissue and neural rosettes representing ectodermal tissue (Figure 16D, H, L). 

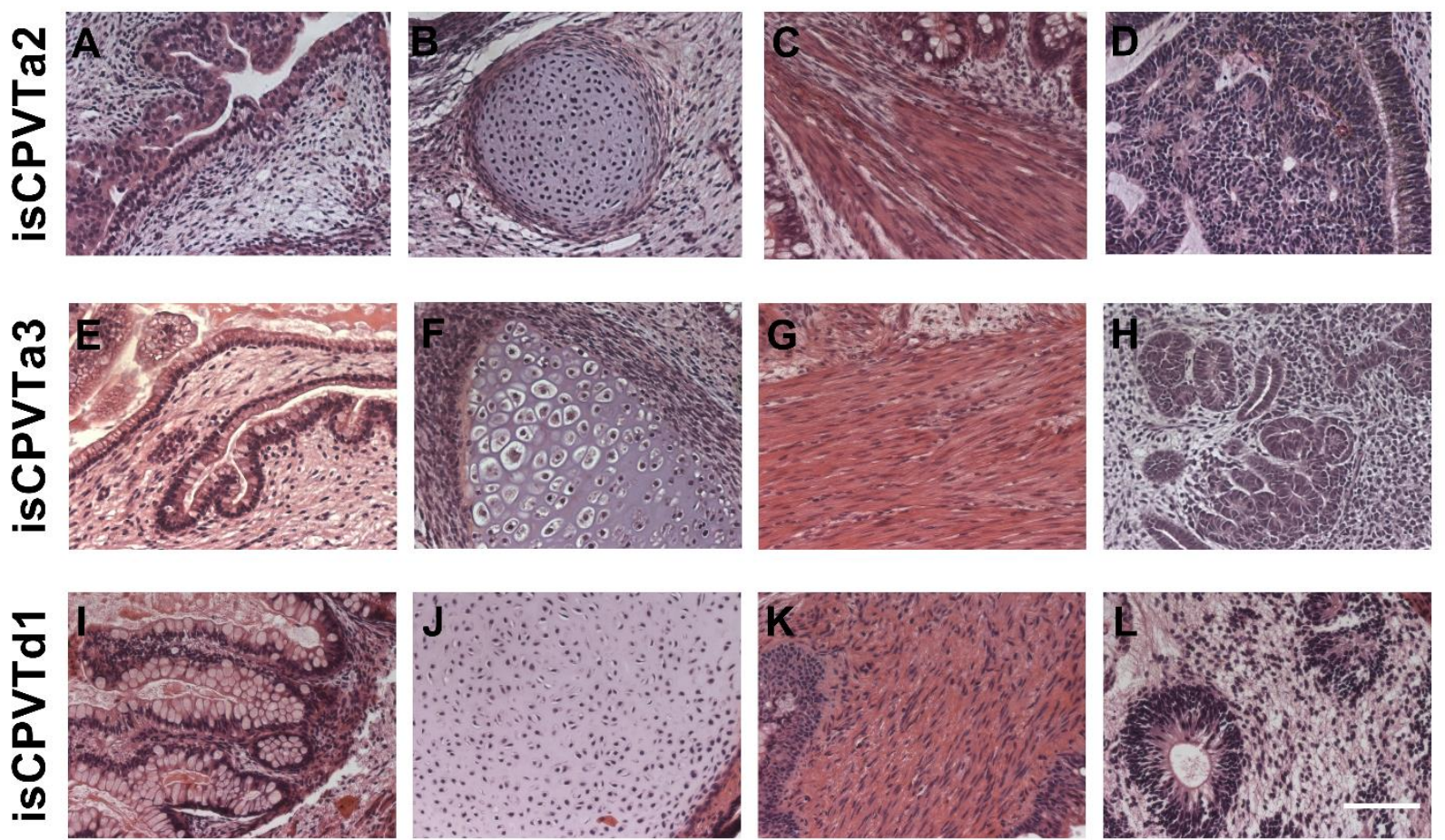

Figure 16. Teratoma formation of hiPSCs after subcutaneous injection into immunodeficient RAGC mice. Teratomas developed from isCPVTa2 (A-D), isCPVTa3 (E-H), and isCPVTd1 (I-L) contained derivatives of all three embryonic germ layers: intestinal tissue with endodermal origin (A, E, $\mathbf{I})$, mesoderm represented by cartilage $(\mathbf{B}, \mathbf{F}, \mathbf{J})$ and muscle cells $(\mathbf{C}, \mathbf{G}, \mathbf{K})$, and ectodermal neural rosettes $(\mathbf{D}, \mathbf{H}, \mathbf{L})$. Scale bar: $100 \mu \mathrm{m}$.

Taken together, the data show that the all generated and analyzed iPSC lines isCPVTa2, isCPVTa3, and isCPVTd1 were pluripotent. They expressed pluripotency-related markers on mRNA and protein levels. They had the potential to differentiate into derivatives of the three germ layers both in vivo and in vitro.

\subsubsection{Karyotyping}

It was reported that iPSCs might develop chromosomal instability under long-term culture conditions (Stadtfeld et al., 2010). The generated hiPSCs in this study were used for differentiation experiments before a maximum of passage 30 . To determine whether these cells exhibit numerical aberrations of their chromosomes, hiPSCs in passage $\geq 30$ were analyzed. A trisomy of chromosome 12 or 17 , which might provide a selective advantage for the propagation 
of undifferentiated cells, was not detected in the generated isCPVTa3-3. Representative diploid karyograms for isCPVTa3 were shown (Figure 17).
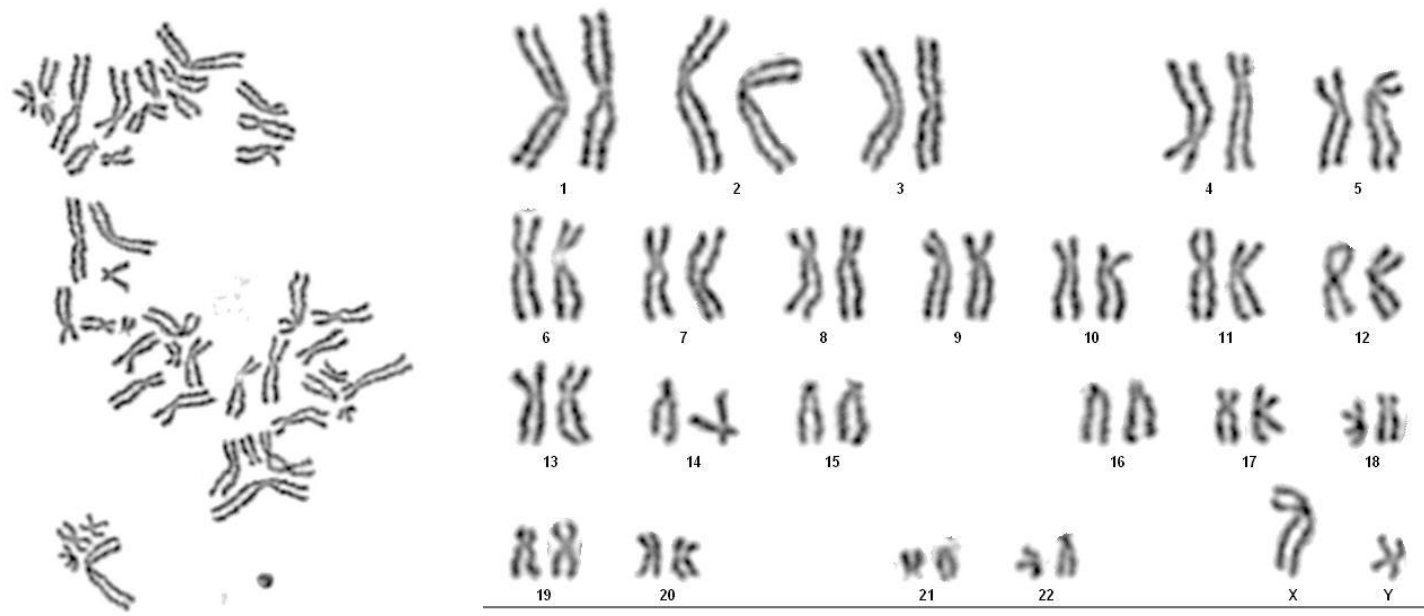

Figure 17. Diploid karyograms for generated hiPSCs. Respectively showed a normal karyotype 46, $\mathrm{XY}$ for isCPVTa3 cells.

\subsubsection{Verification of the RYR2 point mutation in CPVTa- and CPVTd-iPSCs}

$R Y R 2$ gene is located on chromosome 1q43, and has 105 exons. The mutation of CPVTa2 and a3 is located in exon 14, and the mutation of CPVTd1 is located in exon 99. The RYR2 gene region containing the mutant sites found in the CPVTa2, CPVTa3 and CPVTd1 patients were sequenced to confirm the genotypes in CPVT(a2, a3, d1)-iPSCs (Figure 18). The genomic DNA of the generated hiPSCs was isolated and sequenced approximately $150 \mathrm{bp}$ up- and downstream of the predicted mutation positions within the RYR2 gene. All established hiPSCs lines of CPVTa2, CPVTa3 and CPVTd1 showed the mutation R420W and H4742Y, respectively, referred to the coding sequence of the RYR2 transcript variant (NCBI reference sequence: NM_001035.2). For CPVTa2 and a3, one allele carries a cytosine whereas the other allele carries a thymine. For CPVTd1, one allele carries a cytosine whereas the other allele carries a thymine. On the translational level, the missense mutation in CPVTa2 and CPVTa3 leads to the amino acid tryptophan instead of arginine, and in CPVTd1 leads to the amino acid tyrosine instead of histidine. 

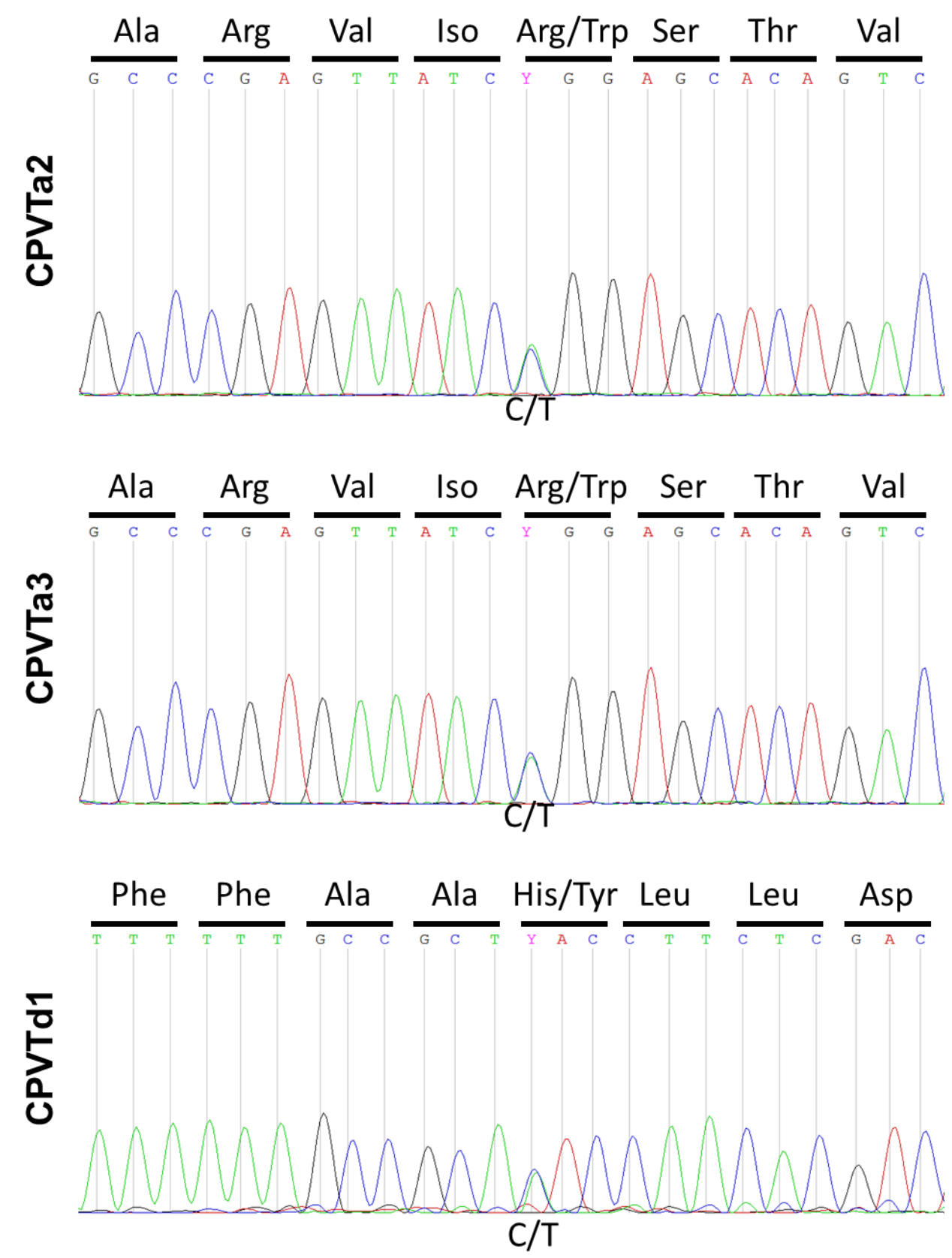

Figure 18. Verification of RYR2 point mutations in CPVTa- and CPVTd-iPSCs. All iPSC lines of CPVTa2 and CPVTa3 exhibited the point mutation on position 1258 referred to the coding sequence of the RYR2 transcript variant (NCBI: NM_001035.2), where a cytosine is replaced by a thymine on one of the alleles. All iPSC lines of CPVTd1 exhibited the point mutation on position 14224 referred to the coding sequence of the $R Y R 2$ transcript variant (NCBI: NM_001035.2), where a cytosine is replaced by a thymine on one of the alleles. The CPVTa2, a3 mutation leads to an amino acid change (arginine to tryptophan). The CPVTd1 mutation leads to an amino acid change (histidine to tyrosine).

\subsection{Generation and electrophysiological characterization of hiPSC-CMs}




\subsubsection{Differentiation of Ctrl- and CPVT-hiPSCs into CMs}

Ctrl- and CPVT-hiPSCs were differentiated into CMs using the cardiac-directed differentiation method (Figure 19A). First beating cells appeared at day 7-8 during directed cardiac differentiation of hiPSCs. The CMs were beating robustly around day 20, 12 days after culturing in the B27 medium.

CMs with purity more than 50\% analyzed by flow cytometry (Figure 19B) and visual observation were chosen for first digestion. The purity of CMs could reach more than $85 \%$ after first digestion by using physical purify skills mentioned before (2.2.1.6). No obvious differences between CPVTa2-, CPVTa3-, CPVTd1- and Ctrl-iPSC-CMs were observed regarding the differentiation efficiency.

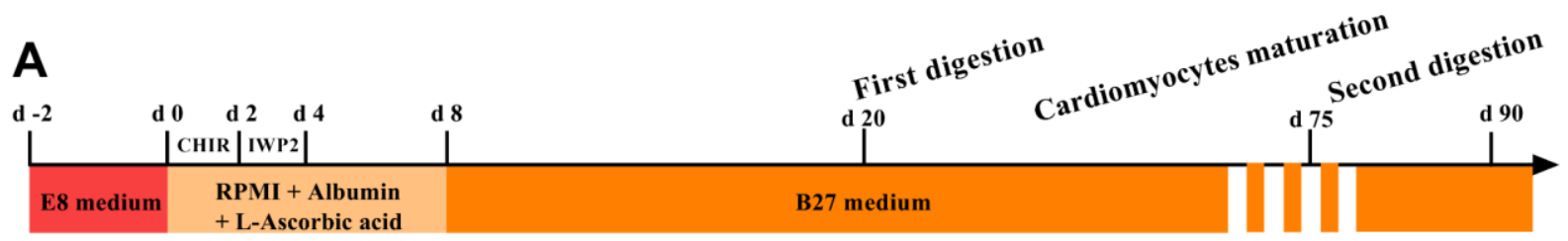

B
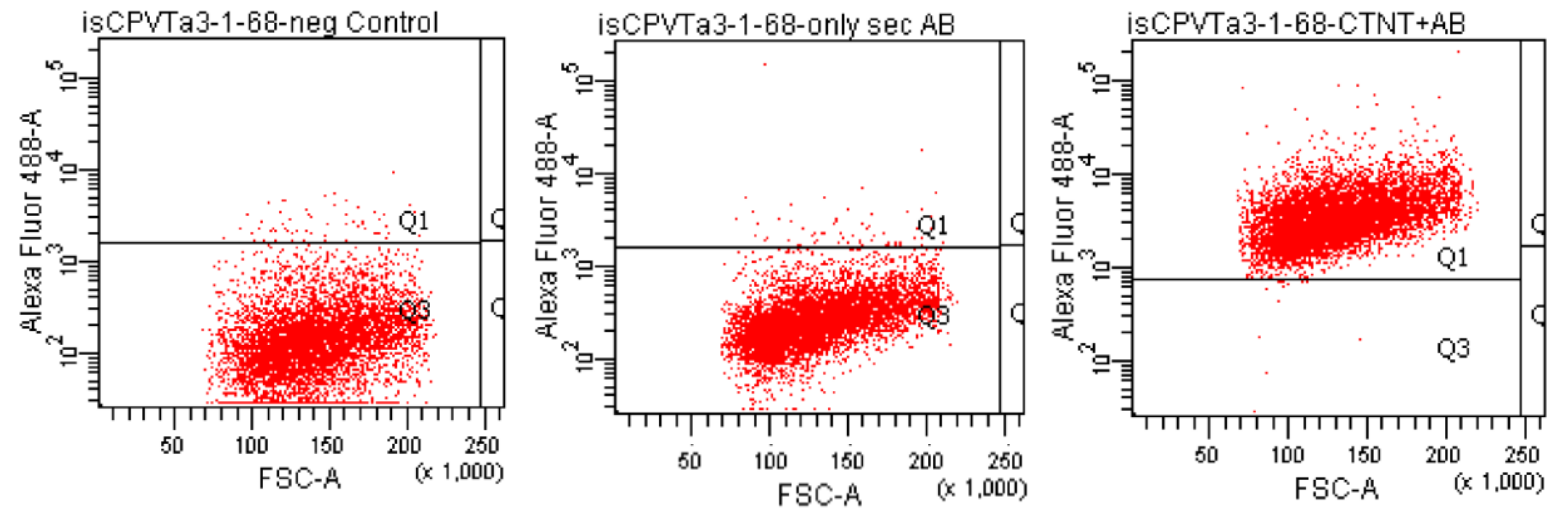

Figure 19. Directed differentiation procedures and differentiation efficiency. (A) Schematic presentation of cardiac-directed differentiation procedures of hiPSCs. (B) Shown are flow cytometry analysis of CMs stained with cTNT, demonstrating CMs used for further maturation-inducing culture with a high purity.

CMs after second digestion showed round, spindle-shaped, tri-, or multi-angular morphologies. The co-immunostaining with antibodies against the myofilament protein $\alpha$-actinin, and the gap junction protein $\mathrm{Cx} 43$ showed that the $\mathrm{CM}$ s can conducting electrical signals between adjacent 
cells. (Figure 20A). After three-month culture, the CMs had a high RYR2 (green) expression (Figure 20B). MLC2a and MLC2v co-immunostaining showed that majority of CMs were positive for MLC2v, indicating that they are ventricular-like CMs (Figure 20C).

No obvious differences of the expression of cardiac-specific proteins between CPVTa2-, CPVTa3-, CPVTd1- and Ctrl-iPSC-CMs were observed.

A
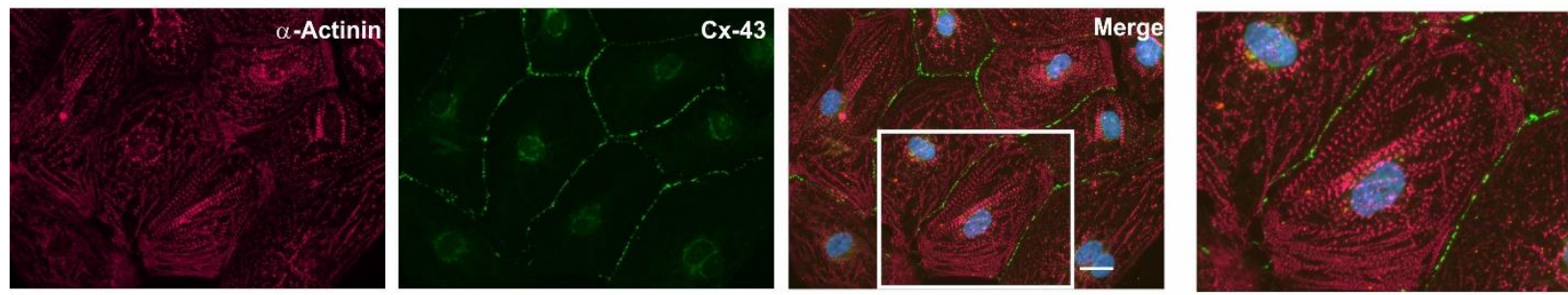

B
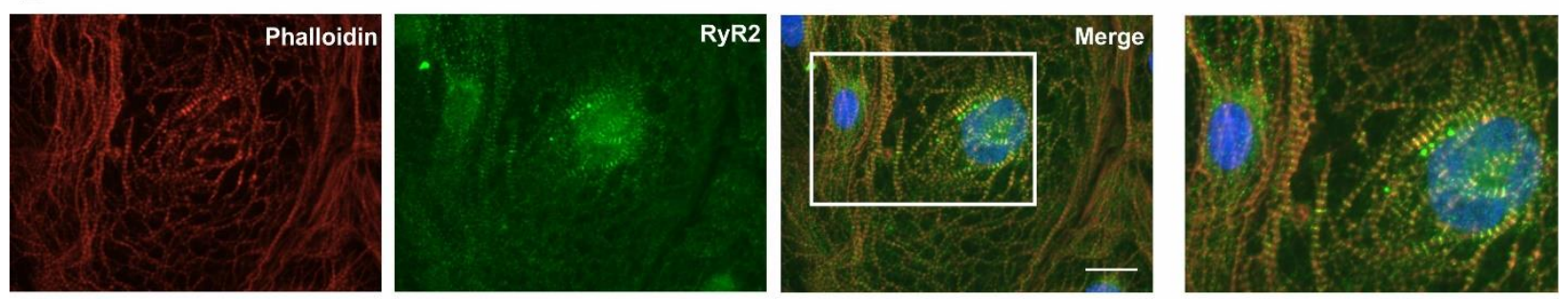

C
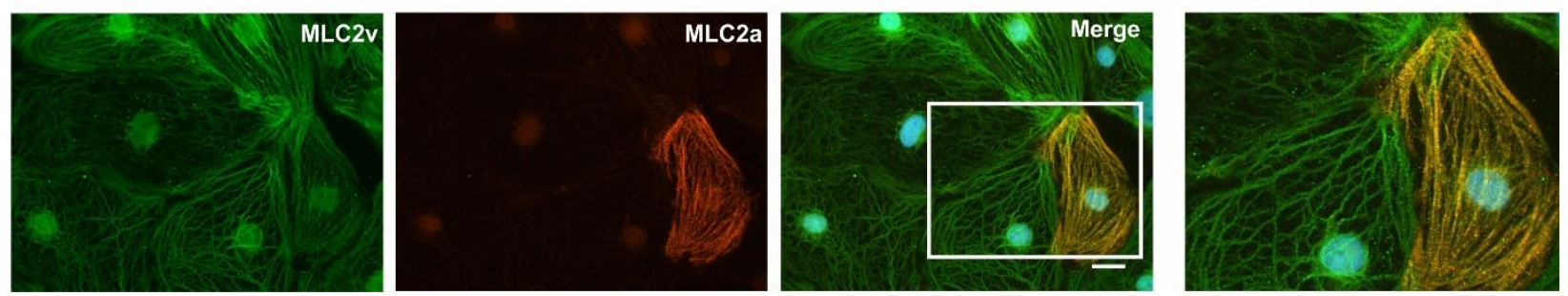

Figure 20. The differentiated CMs show normal sarcomere structure. Representitive coimmunostaining showed that hiPSC-CMs (isCPVTa2-3) expressed structural myofilament protein $\alpha$ actinin (in red) and the gap junction protein $\mathrm{Cx} 43$ (in green) (A), Phalloidin (in red) and RYR2 (in green) (isCPVTa3-1) (B), and MLC2v (in green) and MLC2a (in red) (isCPVTa3-1) (C). Cell nuclei were stained with DAPI (in blue). Scale bar: $20 \mu \mathrm{m}$.

\subsubsection{Action potential morphology of Ctrl-CMs and CPVT-CMs}

In both Ctrl- and CPVT-iPSC-CM cultures, four different types of CMs with typical AP morphologies were detected by using the patch clamp technique (Figure 21). The typical APs of ventricular-like and atrial-like CMs were characterized by a relative negative RMP $(\leq-60 \mathrm{mV})$, 
a rapid AP upstroke, an APA bigger than $90 \mathrm{mV}$. The ventricular-like CMs show a prominent plateau phase while atrial-like CMs lack the plateau phase, leading to a triangular-like shape. The parameter APD90/APD50 $\geq 2$ for atrial CMs was used to distinguish ventricular-like from atriallike CMs (Fatima et al., 2011a). A nodal-like AP exhibited typically a less negative RMP ( $\geq-55$ $\mathrm{mV}$ ), an APA smaller than $85 \mathrm{mV}$ and a very slow maximal upstroke velocity (Vmax $\leq 5 \mathrm{~V} / \mathrm{s}$ ). Purkinje-like CMs were defined as prolonged APD especially for APD90 (Pallante et al., 2010). Typical morphologies of ventricular-like, nodal-like, atrial-like and Purkinje-like CMs were shown as following (Figure 21).
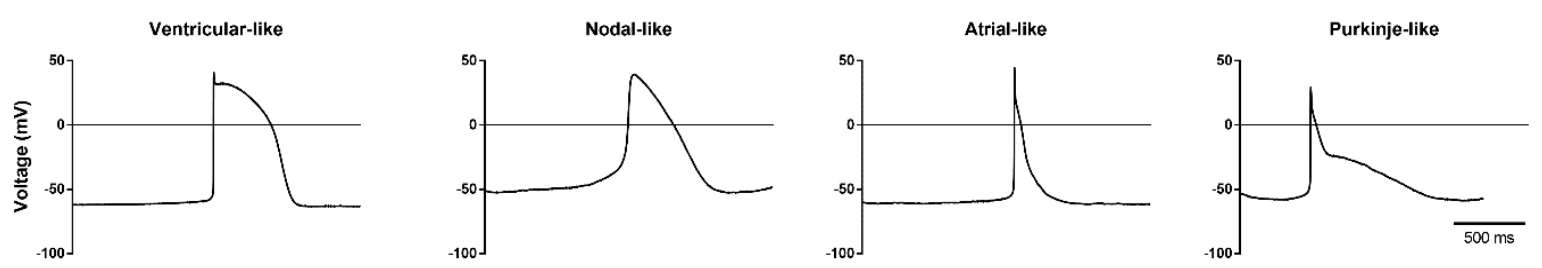

Figure 21. Classification of differentiated CMs according to their AP morphologies. The APs of ventricular-like and atrial-like CMs were characterized by a rapid AP upstroke and a relative negative RMP. The difference between ventricular-like and atrial-like CMs was that ventricular-like CMs have a long plateau whereas atrial-like CMs show a triangular-like shape. The typical nodal-like CMs had a less negative resting membrane potential, a slower upstroke velocity, a smaller AP amplitude. The APDs (especially APD90) were prolonged in Purkinje-like CMs.

Using the cardiac-directed differentiation method, the majority of the whole-cell patched cells (88-97\%) were found to be ventricular-like cells in both Ctrl- and CPVT-iPSC-CM cultures (Figure 22) without significant differences among the cell lines analyzed. On average, less than $4.2 \%$ (range $0-6.5 \%$ ) of CMs were atrial-like CMs, whereas less than 3.3\% (range 0-9\%) of CMs were pacemaker-like CMs. In addition, the Purkinje-like CMs were very rare using this directed differentiation protocol. Only one Purkinje-like CM (APD90/APD50=7.7) was observed in CPVTc2-iPSC-CMs group. No statistical differences were observed between Ctrl- and CPVTiPSC-CM cultures regarding the CM subtype specification. Because CPVT was primarily caused by the abnormality in ventricular $\mathrm{CMs}$, the directed differentiation and maturation-inducing protocol used in this study provides a reliable cell source of ventricular CMs that can be used for in vitro disease modeling of CPVT. 


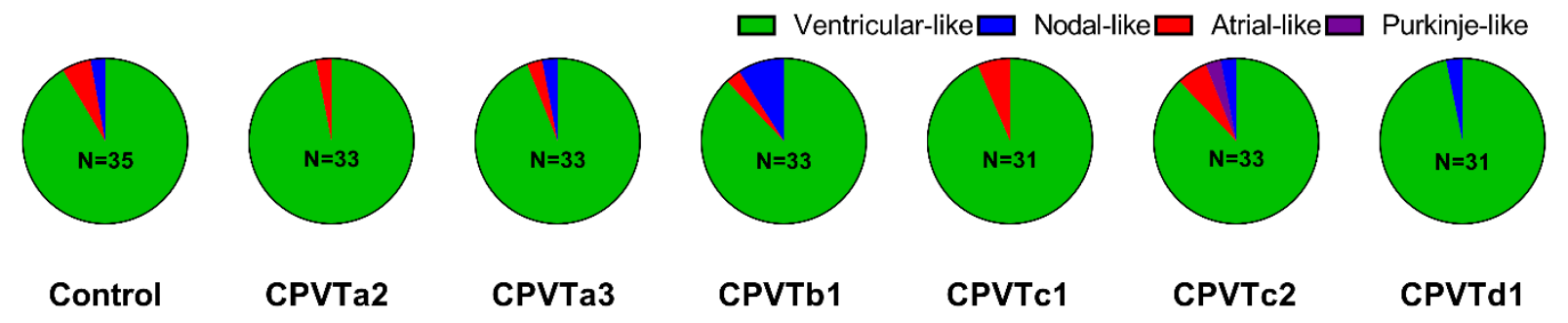

Figure 22. Percentage of ventricular-, atrial-, nodal-like and Purkinje-like CMs in Ctrl- and CPVTCMs. The majority of Ctrl- and CPVT-CMs (>87.88\%) was ventricular-like CMs ( $\mathrm{n}=31-35$ cells from each patient), based on single cell electrophysiological measurements of APs.

To find out whether the different AP parameters of ventricular-like CMs were different in CtrlCMs and CPVT-CMs, APs of single CMs were recorded in 3-month-old Ctrl-CMs and CPVTCMs (Figure 23A). For each patient, three independent iPSC lines were used for cardiac differentiation and phenotype characterization in order to avoid the difference due to one specific cell line.

All the parameters were automatically quantified by using Lab chart software as described in 2.2.8. There was no significant difference in APA, ranged from $96.28 \mathrm{mV}$ to $101.9 \mathrm{mV}$ between Ctrl-CMs and CPVT(a2, a3, b1, c1, c2, d1)-CMs, respectively (Figure 23B). Compared to CtrlCMs $(-63.13 \pm 0.83, \mathrm{n}=32)$, the RMP was significantly more positive in CPVTa3-CMs $(-60.45 \pm$ 0.57, $\mathrm{n}=31)$, and CPVTd1-CMs (-59.83 \pm 0.7, $\mathrm{n}=31)$, whereas CPVTa2-CMs showed also more positive (slightly but not significantly) RMP $(-61.17 \pm 0.59, \mathrm{n}=32, \mathrm{p}=0.06)$. No significant difference in RMP was observed between Ctrl-CMs and CPVT(b1, c1, c2)-CMs, respectively (Figure 23C).

However, the $\mathrm{V}_{\max }$ were significant lower in CPVTa2-CMs $(17.41 \pm 1.17 \mathrm{~V} / \mathrm{s}, \mathrm{n}=32)$, CPVTa3CMs $(17.75 \pm 1.21 \mathrm{~V} / \mathrm{s}, \mathrm{n}=31)$, CPVTb1-CMs (17.34 $\pm 1.35 \mathrm{~V} / \mathrm{s}, \mathrm{n}=29)$, CPVTc2-CMs (17.87 \pm $1.42 \mathrm{~V} / \mathrm{s}, \mathrm{n}=29)$ and CPVTd1-CMs (11.52 $\pm 0.98 \mathrm{~V} / \mathrm{s}, \mathrm{n}=31)$ comparing to Ctrl-CMs $(21.47 \pm$ $1.06 \mathrm{~V} / \mathrm{s}, \mathrm{n}=32$ ). No significant difference in $\mathrm{V}_{\max }$ was observed between Ctrl-CMs and CPVTc1-CMs (21.13 $\pm 0.89 \mathrm{~V} / \mathrm{s}, \mathrm{n}=29)$ (Figure 23D).

Because APD is related to frequency, only the whole-cell patched cells stimulated by the patchclamp generated pacing impulse were used for APD analysis. Due to the appearance of EADs in CPVTd1-CMs, it was impossible to pace the CPVTd1-CMs with $0.8 \mathrm{~Hz}$, whereas other iPSCCMs were paced at $0.8 \mathrm{~Hz}$. It was observed that APD50 (1193 $\pm 97.12 \mathrm{~ms}, \mathrm{n}=22)$ and APD90 
(1959 $\pm 204.4 \mathrm{~ms}, \mathrm{n}=22)$ of CPVTd1-CMs paced with $0.2 \mathrm{~Hz}$ were significantly longer than APD50 (231.5 $\pm 23.98 \mathrm{~ms}, \mathrm{n}=14)$ and APD90 (309.3 $\pm 26.26 \mathrm{~ms}, \mathrm{n}=14)$ of Ctrl-CMs paced with $0.2 \mathrm{~Hz}$, correspondently (Figure 23E). 
A

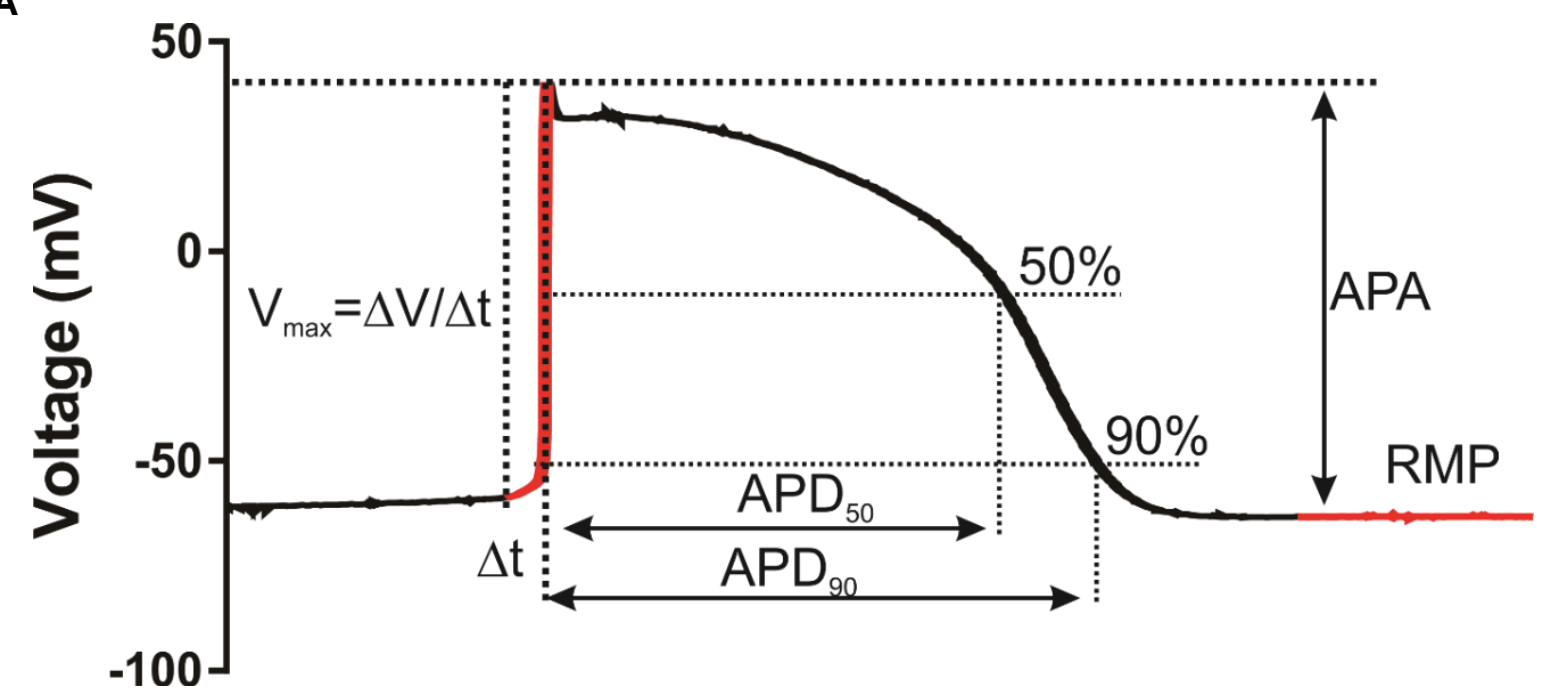

B

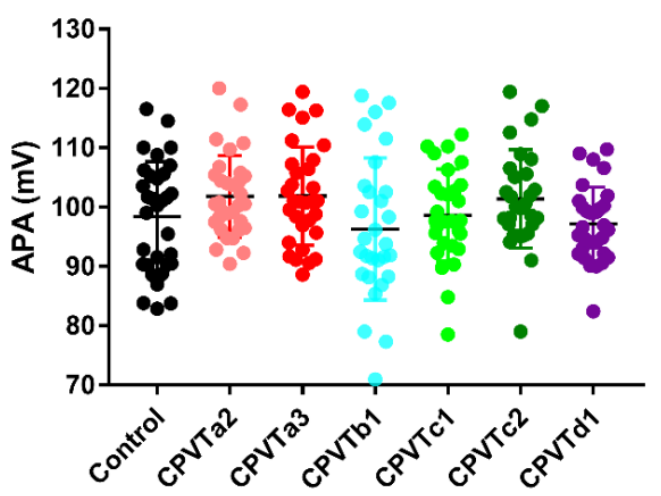

C

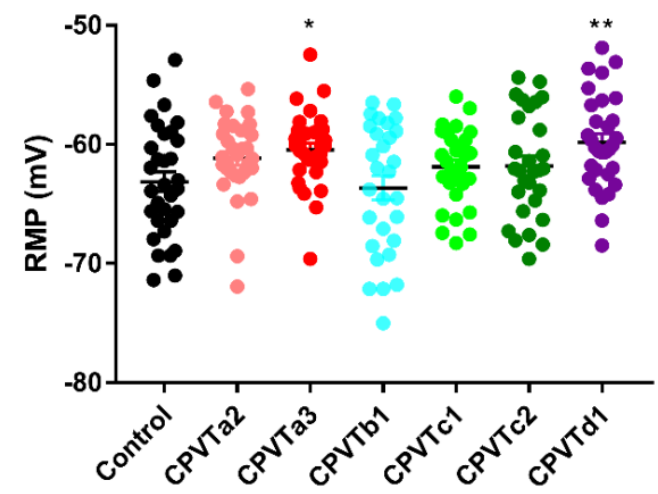

D

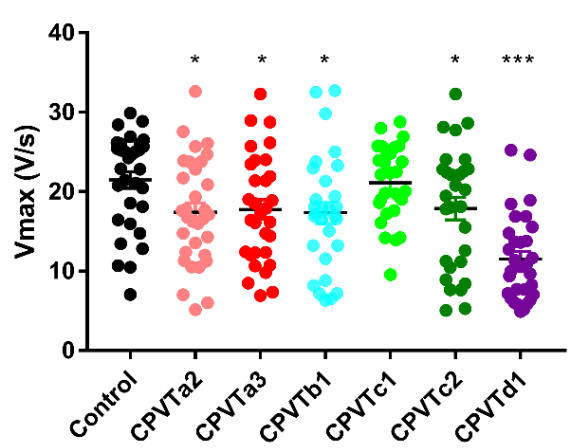

E

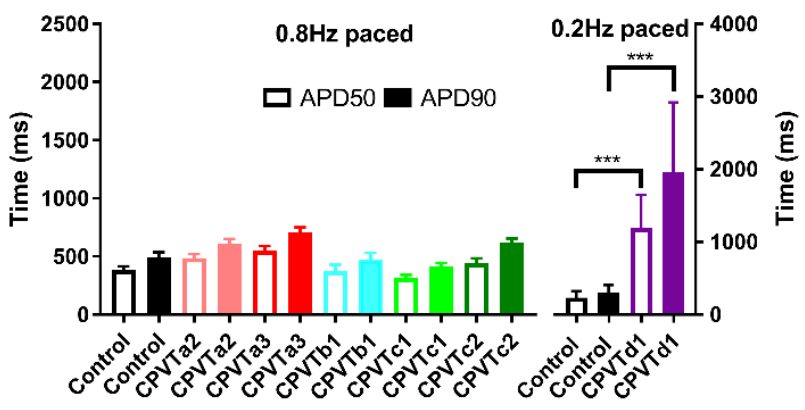

Figure 23. Action potential characteristics of ventricular-like CMs. (A) The cartoon illuminated the measured parameters: APA, RMP, $\mathrm{V}_{\max }$, APD50, and APD90. Statistic analysis of the AP parameters of ventricular-like Ctrl-CMs and CPVT-CMs in APA (B), RMP (C), $\mathrm{V}_{\max }(\mathbf{D})$, APD50/90 (E). Data are presented as mean \pm SEM. For $0.8 \mathrm{~Hz}$ paced APD analysis experiments, cell numbers of Ctrl-CMs and CPVT(a2, a3, b1, c1, c2)-CMs equal to 16, 19, 17, 9, 12 and 14, respectively. For $0.2 \mathrm{~Hz}$ pacing experiments, 14 Ctrl-CMs and 22 CPVTd1-CMs were analyzed. 


\subsubsection{The characteristics of CPVT-CMs in basal condition}

CPVT was associated with increased incidences of ventricular tachycardia and cardiac arrhythmias, which can be initiated by DAD- and EAD-induced TA. To investigate if there were differences in the severity of the arrhythmias in non-ISO stimulated Ctrl-CMs and CPVT-CMs, four prominent irregularities during spontaneous AP recordings in CPVT-CMs were compared to Ctrl-CMs: DADs, EADs, DAD- or EAD-induced TAs (Figure 24).

Under the basal condition, only $8 \%$ of Ctrl-CMs ( $n=36$ cells from 2 controls) showed arrhythmic beating and putative EADs or DADs and/or TAs, while about 20\% for CPVTa2-CMs $(\mathrm{n}=35)$, 16\% CPVTa3-CMs (n=38), 18\% CPVTb1-CMs (n=38), 30\% for CPVTc1-CMs (n=36), 43\% for CPVTc2-CMs $(n=35)$ and 55\% for CPVTd1-CMs $(n=31)$ were observed to exhibit arrhythmic beating including DADs, EADs and TAs. Since no significant differences were observed between patient $\mathrm{a} 2$ and $\mathrm{a} 3$, as well as between patient $\mathrm{c} 1$ and $\mathrm{c} 2$, for further analysis, the data from a 2 and a3, or from c1 and c2 were pooled (Figure 24B).

In the non-ISO stimulated condition, only CPVTd-CMs showed unique EADs and EADinduced TAs (Figure 24A), but the DADs and DAD-triggered activities were appeared in all non-ISO-stimulated groups no matter Ctrl-CMs or CPVT(a, b, c, d)-CMs. After quantification, only the number of DADs and TAs in CPVTd-CMs $(8.9 \pm 2.53, \mathrm{n}=31)$ was significantly higher than in Ctrl-CMs $(1.9 \pm 1.16, \mathrm{n}=36)$ (Figure 24B). Furthermore, the number of EADs and TAs in CPVTd-CMs $(56.01 \pm 24.14, \mathrm{n}=31)$ was significantly higher than in Ctrl-CMs $(0 \pm 0, \mathrm{n}=36)$ (Figure 24B).

The beating frequencies were also analyzed. In comparison with Ctrl-CMs $(0.49 \pm 0.06 \mathrm{~Hz})$, the beating frequency was significantly lower for CPVTa-CMs $(0.23 \pm 0.03 \mathrm{~Hz})$ and CPVTd-CMs $(0.15 \pm 0.02 \mathrm{~Hz})$ but not for CPVTb-CMs $(0.50 \pm 0.05 \mathrm{~Hz})$ and CPVTc-CMs $(0.55 \pm 0.03 \mathrm{~Hz})$ (Figure 24B). 
A
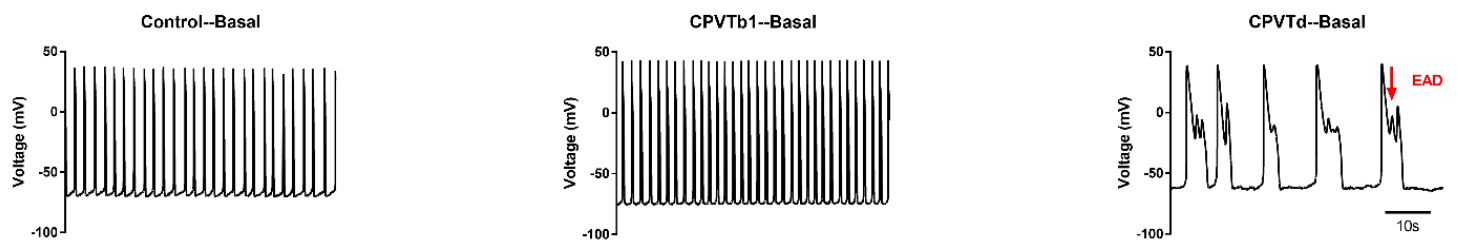

B
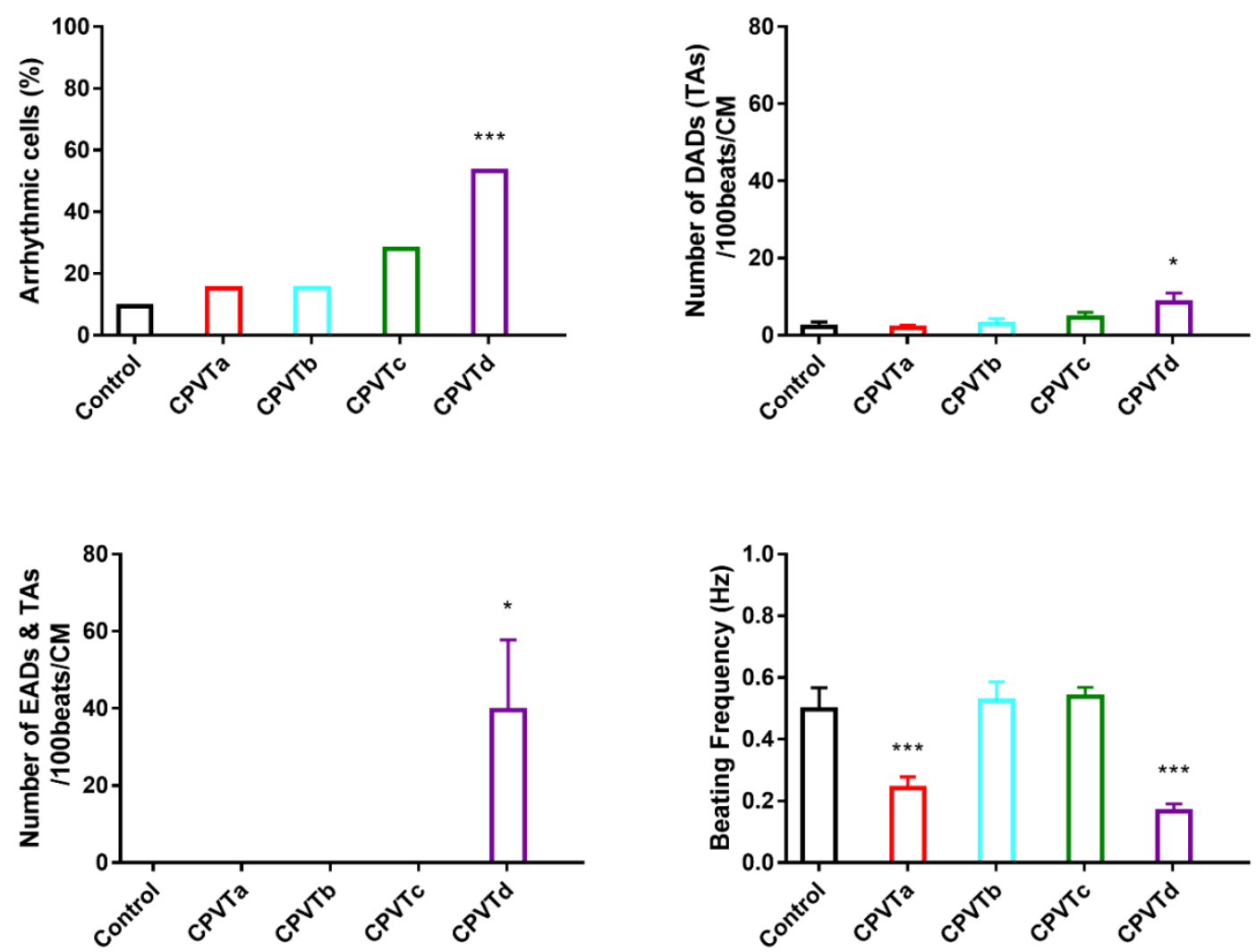

Figure 24. Patch-clamp results show EADs in non-ISO stimulated CPVTd-CMs. The representitive original traces of whole-cell patch-clamp recording for Ctrl-CMs, CPVT(a, b, c)-CMs and CPVTd-CMs, respectively are shown (A). Statistical parameters of arrhythmic cells, the number of DADs and TAs and the number of EADs and TAs showed unique significantly difference between Ctrl-CMs and CPVTd-CMs but not for CPVT(a, b, c)-CMs (B). Compared with Ctrl-CMs, the beating frequency was significantly lower for CPVTa-CMs and CPVTd-CMs (B). For the non-ISO stimulated experiments, numbers of Ctrl-CMs and $\operatorname{CPVT}(\mathrm{a}, \mathrm{b}, \mathrm{c}, \mathrm{d})-\mathrm{CMs}$ equal to 36, 73, 38, 71 and 31, respectively. 
MEA technology enables non-invasive measurements of local field potentials (Figure 25A) of electrically active cells and thus the activity of the underlying ion channels. MEA has been successfully used to study a homogeneous and electrically coupled population of CMs, and showed a similar ECG waveform. Every well of six-well MEA has nine electrodes (Figure 25A). The monolayer sheet of CMs (Figure 25A bottom right corner) was chosen for recording their field potentials. The original traces of nine electrodes displayed standard field potentials of the CMs in the well (also counts as one culture), which showed beating in the same rhythm (Figure 25B).

A

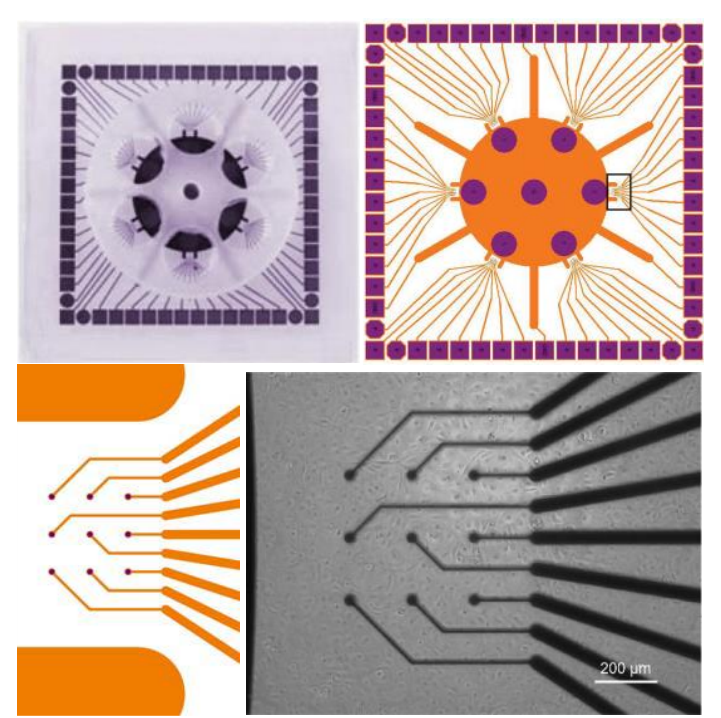

B

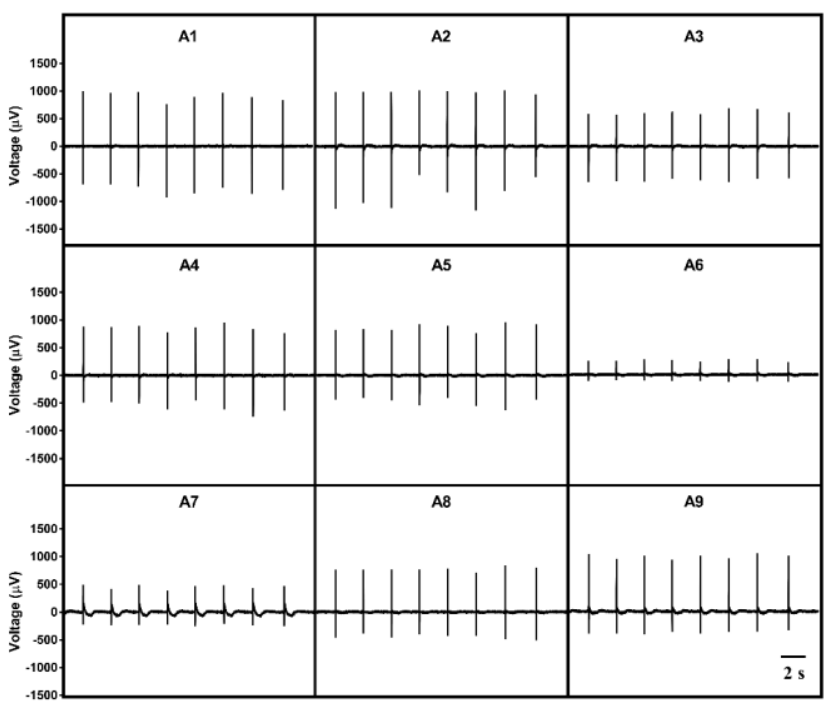

Figure 25. The MEA technology is used to quantify arrhythmic events. The entity and layout of six-well MEA and the CMs were seeded as monolayer after second digestion (A). The traces of nine electrodes displayed standard field potentials and the CMs in the well (also counts as one culture) showed beating in the same rhythm $(\mathbf{B})$.

Lan et al. showed analysis of putative DADs by MEA and also provided the procedures to quantify arrhythmic events by using this technology (Lan et al., 2013). However, different from Lan et al. findings, PVC-like (Figure 26) and VT-like events (Figure 26) were observed in this study. 


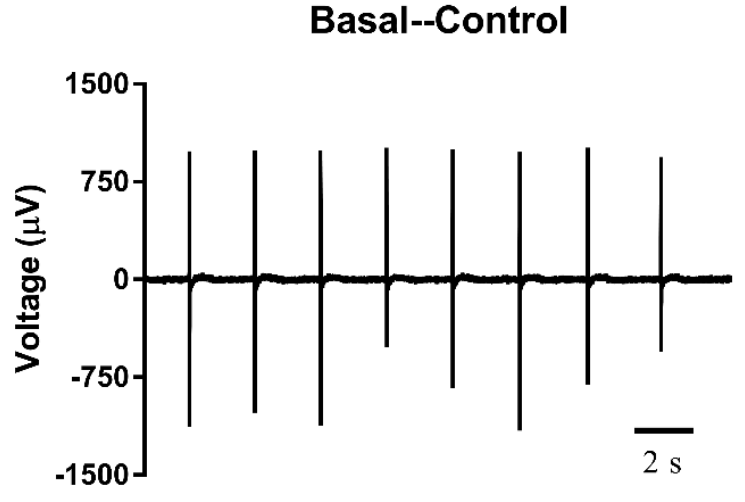

Basal--CPVTd

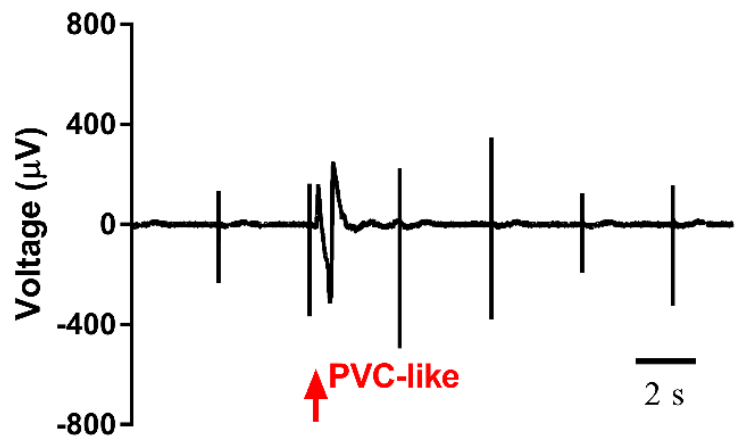

Basal--CPVTa2

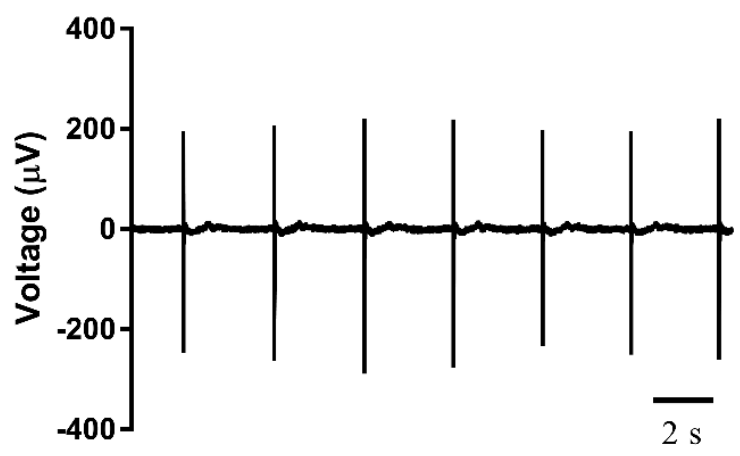

Basal--CPVTd

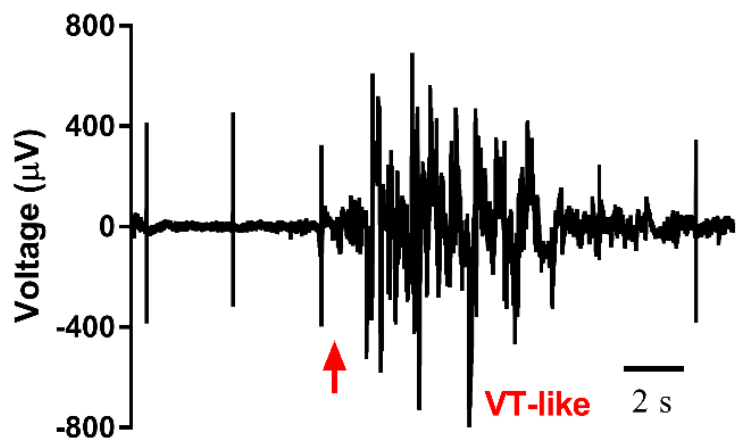

Figure 26. The representative original traces of MEA recording for $\mathrm{Ctrl-CMs,} \mathrm{CPVT(a,} \mathrm{b,} \mathrm{c)-CMs}$ and CPVTd-CMs, respectively. The PVC-like and VT-like events are indicated with a red arrow.

In the non-ISO stimulated condition, only $1.8 \%$ of the monolayer cultures of Ctrl-CMs $(\mathrm{n}=108$ from 2 cell lines per control, 2 controls, and 18 experiments) showed arrhythmic beating including PVC- or VT-like events. Similar results were observed for CPVTa-CMs (3.3\%; n=92 from 3 cell lines per patient, 2 patients, and 16 experiments), CPVTb-CMs (3.9\%; n=77 from 2 cell lines, and 13 experiments) and CPVTc-CMs (5.8\%; n=104 from 2-4 cell lines per patient, 2 patients, and 18 experiments). In contrast, 20.2\% of the monolayer cultures of CPVTd-CMs ( $\mathrm{n}=84$ from 3 cell lines, and 14 experiments) showed arrhythmic beating including PVC- or VTlike events (Figure 27A). Number of PVC-like events in CPVTd-CMs were significantly higher when compared to Ctrl-CMs. Nevertheless, the Number of PVC-like events in CPVT(a, b, c)CMs had no significantly differences in comparison to Ctrl-CMs (Figure 27B). Similarly, the VT duration was significantly longer only in CPVTd-CM cultures, but not in CPVTa-, CPVTb-, 
CPVTc-CM cultures when compared to Ctrl-CM cultures under basal conditions (Figure 27C). In addition, compared to Ctrl-CMs, the beating frequency was significantly lower in CPVTaCMs, CPVTc-CMs, and CPVTd-CMs but significantly higher in CPVTb-CMs under basal conditions (Figure 27D). 
A
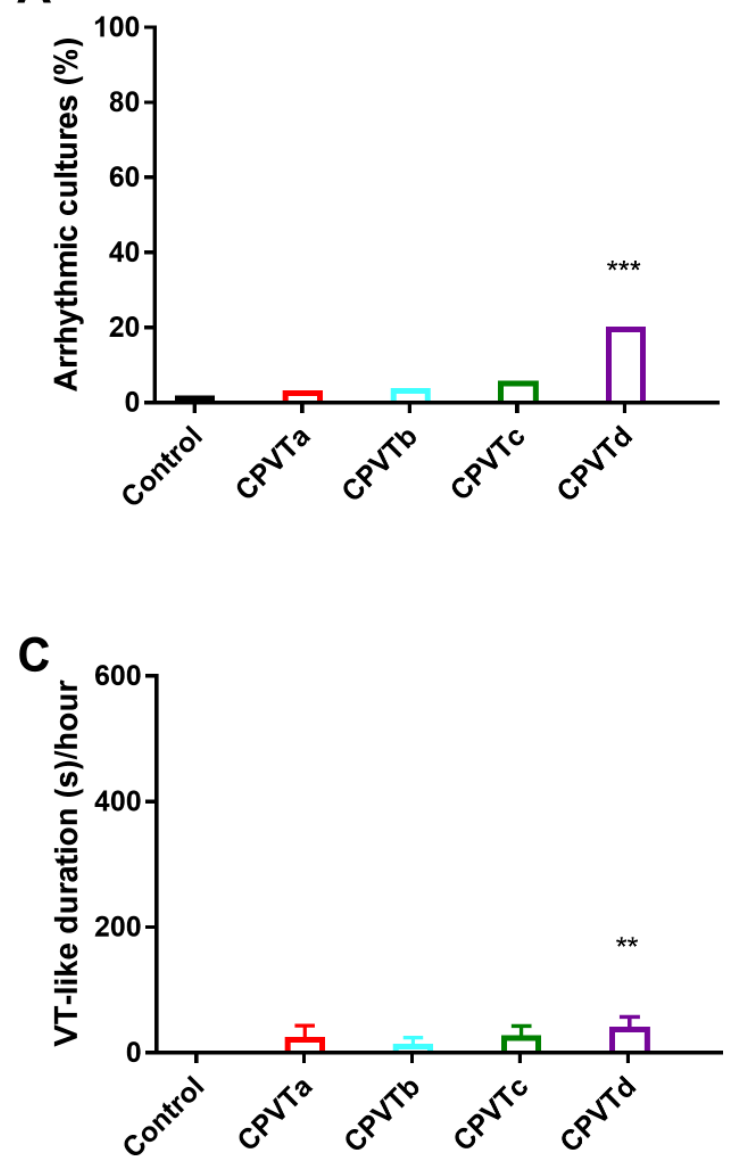

B

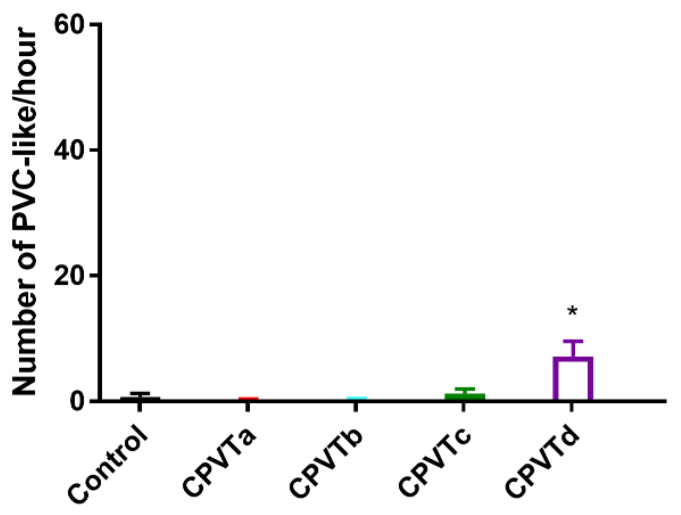

D

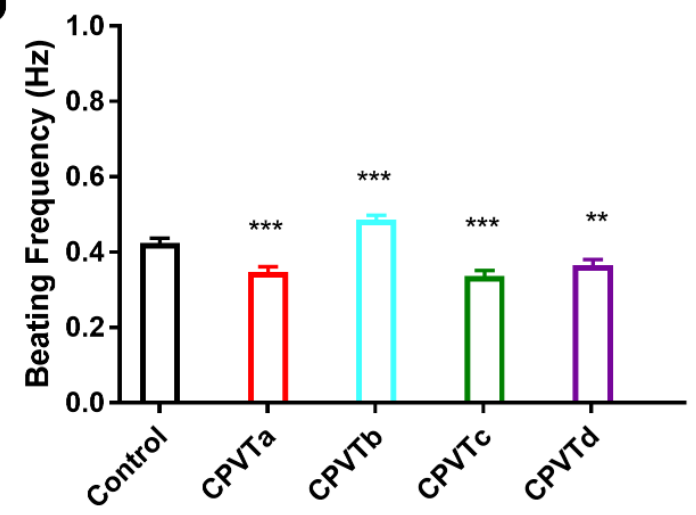

Figure 27. MEA results presented arrhythmic events in non-ISO stimulated CPVTd-CMs. Statistical parameters of arrhythmic cultures, the number of PVC-like events, and VT-like duration show unique significant differences between Ctrl-CMs and CPVTd-CMs but not for CPVT(a, b, c)-CMs (A, B, C). Compared with Ctrl-CMs, the beating frequency is significantly lower for CPVTa-CMs, CPVTcCMs, and CPVTd-CMs, but higher for CPVTb-CMs (D). For the non-ISO stimulated MEA experiments, culture numbers of Ctrl-CMs and CPVT(a, b, c, d)-CMs equal to 108, 92, 77, 104, and 84, respectively.

\subsubsection{CPVT-CMs after ISO stimulation recapitulated the disease phenotype of CPVT}

Because arrhythmias in CPVT manifest after exercise or emotional stress, the next step evaluates whether the activation of the $\beta$-AR pathway in CPVT-CMs can recapitulate the disease phenotype of CPVT. The Ctrl-CMs show regular beating after ISO stimulation. After $100 \mathrm{nM}$ ISO stimulation, $\operatorname{CPVT}(\mathrm{a}, \mathrm{b}, \mathrm{c})$-CMs presented dozens of DADs and DAD-induced TAs while the CPVTd-CMs still presented EADs and EAD-induced TAs after ISO stimulation (Figure 28). 

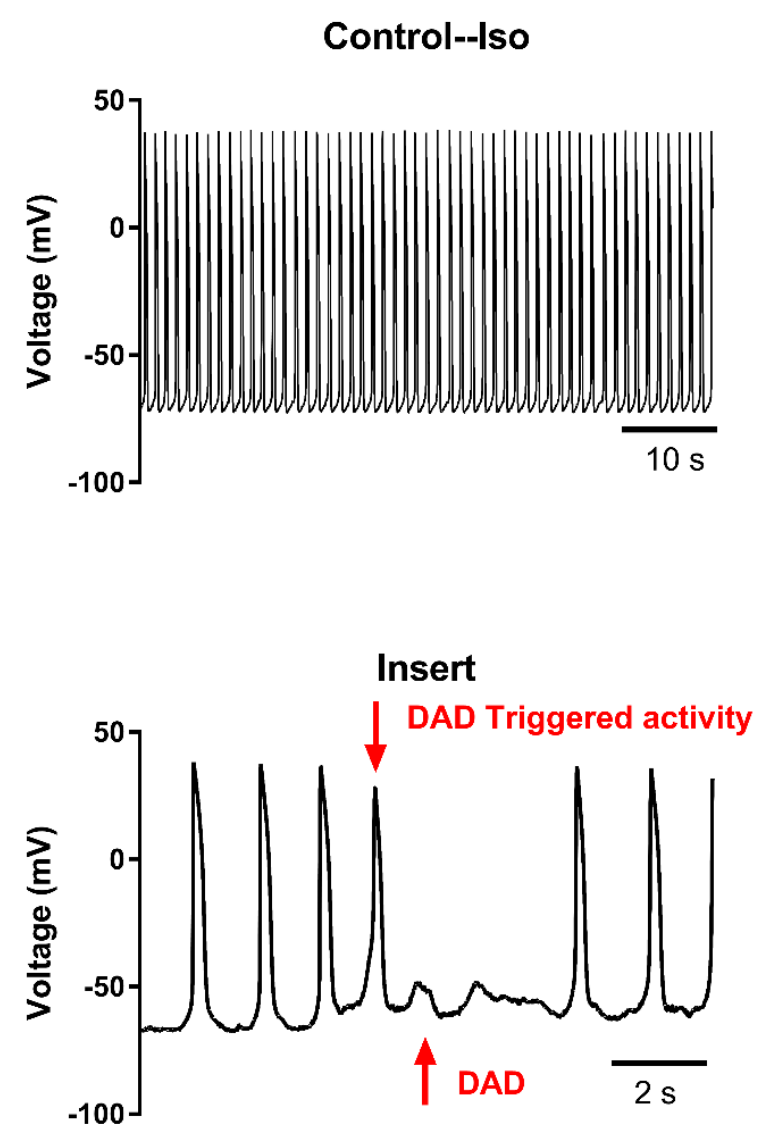
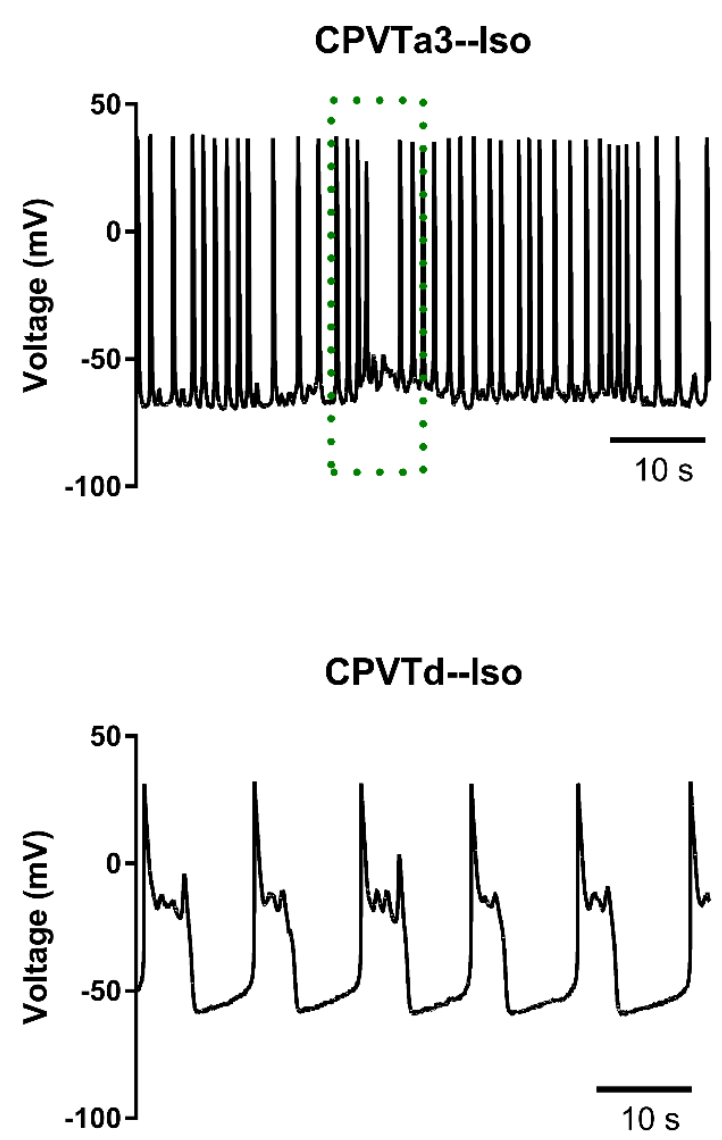

Figure 28. CPVT-CMs presented DADs, EADs and TAs after ISO stimulation. The Ctrl-CMs show regular beating after ISO stimulation. The representative CPVTa3-CM presented dozens of DADs and DAD induced TAs after ISO stimulation. The CPVTd-CMs still presented EADs after ISO stimulation.

First, patch clamp analyses showed that the percentage of arrhythmic cells significantly increased in CPVTa-CMs (84.3\%, n=32), CPVTb-CMs (93.7\%, n=16), CPVTc-CMs (94.6\%, $\mathrm{n}=37)$ and CPVTd-CMs (93.7\%, $\mathrm{n}=16)$ when compared to their respective basal conditions and to Ctrl-CMs after ISO stimulation (Figure 29). To further assess arrhythmia severity, the number of DADs and DAD-induced TAs together with the number of EADs and EAD-induced TAs were quantified. The number of DADs and DAD-induced TAs significantly increased in ISO-treated CPVTa-CMs (44.38 \pm 9.93, $n=32)$, CPVTb-CMs (63.89 $\pm 13.9, n=16)$, and CPVTc-CMs (48.77 $\pm 7.1, \mathrm{n}=37)$ but not in Ctrl-CMs $(1.06 \pm 0.68, \mathrm{n}=22)$ and CPVTd-CMs $(8.58 \pm 3, \mathrm{n}=16)$, suggesting that there might be different arrhythmogenesis mechanisms caused by specific mutations (Figure 29). EADs and EAD-induced TAs especially occurred only in CPVTd-CMs even after challenged with ISO, which further confirmed the mutation-specific arrhythmogenesis 
mechanisms. The beating frequencies of Ctrl-CMs and CPVT(a, b, c, d)-CMs showed a very complex profile due to the existence of lots of DADs and EADs. The ISO treatment resulted in increased beating frequency in Ctrl- and CPVTa- and CPVTd-CMs, but not in CPVTb- and CPVTc-CMs (Figure 29). The not-increased beating frequency in CPVTb- and CPVTc-CMs after the ISO treatment is due to the strongly induced irregular beating, including the stopped beating (Figure 28, Figure 29).
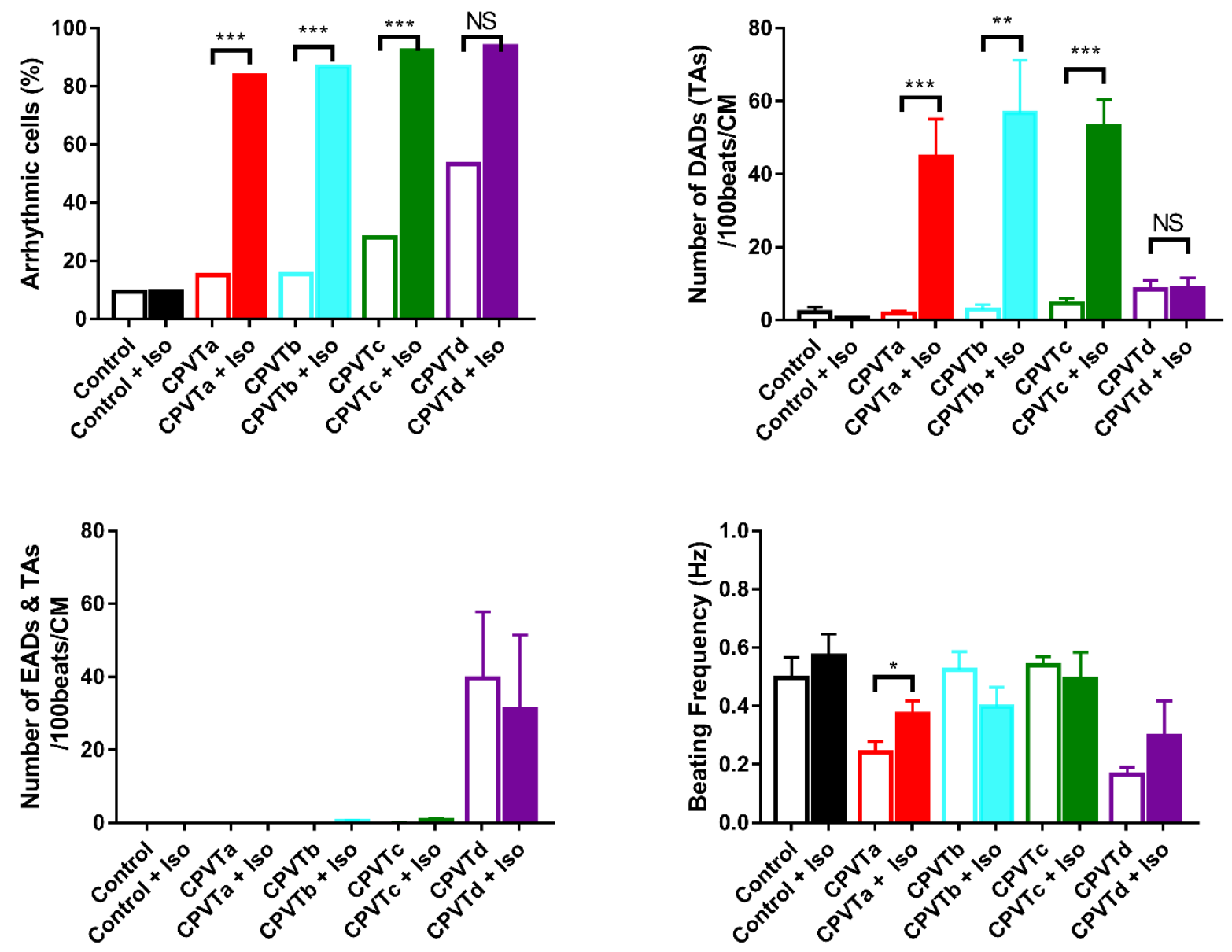

Figure 29. Mutation-specific profiles of DADs, EADs and TAs after ISO stimulation. Shown are comparison of arrhythmic cells, numbers of DADs and TAs, EADs and TAs, and beating frequency in Ctrl-CMs and CPVT(a, b, c, d)-CMs after ISO-treatment. For the ISO-challenged experiments, numbers of Ctrl-CMs and CPVT(a, b, c, d)-CMs equal to 22, 32, 16, 37 and 16, respectively.

Second, MEA recordings were performed in Ctrl- and CPVT-CMs after ISO (100 nM) stimulation. Representative traces of MEA recordings were shown for ISO-treated CPVT-CMs 
which exhibited PVC-like and VT-like events, whereas Ctrl-CMs displayed normal waveforms (Figure 30A).

Application of $100 \mathrm{nM}$ ISO for 15 minutes, the same concentration using for patch-clamp experiments, significantly increased the percentage of arrhythmic cultures in CPVTa-CMs (54.3\%, n=89), CPVTb-CMs (48\%, n=77), CPVTc-CMs (54.8\%, $\mathrm{n}=97)$ and CPVTd-CMs (50\%, $\mathrm{n}=83)$ compared to their corresponding basal conditions or to ISO-stimulated Ctrl-CMs (3.8\%, $\mathrm{n}=108$ ) (Figure 30B). $\beta$-adrenergic stimulation was also found to severely exacerbate the presentation of PVC-like events in MEA recordings in CPVTa-CMs (24.66 $\pm 4.264, \mathrm{n}=92)$, CPVTb-CMs (21.62 $\pm 4.03, n=77)$, CPVTc-CMs (27.54 $\pm 4.74, n=104)$ and CPVTd-CMs (15.36 $\pm 3.037, \mathrm{n}=84)$ but not in Ctrl-CMs $(1.27 \pm 0.9, \mathrm{n}=108)$.

In addition, the ISO treatment resulted in the significantly longer duration of VT-like events in CPVTa-CMs (262.2 \pm 68.69 s/hour), CPVTb-CMs (174.4 \pm 62.44 s/hour), CPVTc-CMs (292.5 \pm 83.48 s/hour) and CPVTd-CMs (352.7 \pm 93.04 s/hour) when compared to their corresponding basal conditions (Figure 30B).

It is interesting to notice that the frequencies of CPVTa-CMs $(0.656 \pm 0.016 \mathrm{~Hz})$ and CPVTdCMs $(0.68 \pm 0.01 \mathrm{~Hz})$ were significantly lower compared to Ctrl-CMs $(0.82 \pm 0.007 \mathrm{~Hz})$ in the ISO-treated conditions even though all CPVT(a, b, c, d)-CMs and Ctrl-CMs showed dramatically increased frequencies after the ISO treatment in relation to their corresponding basal conditions (Figure 30B).

These findings are correspond with the patch-clamp results, which confirm that the MEA technology can be additionally used to investigate the arrhythmia generated by CMs derived from patients-specific CPVT-iPSCs and for the effect of drugs tested. 
A
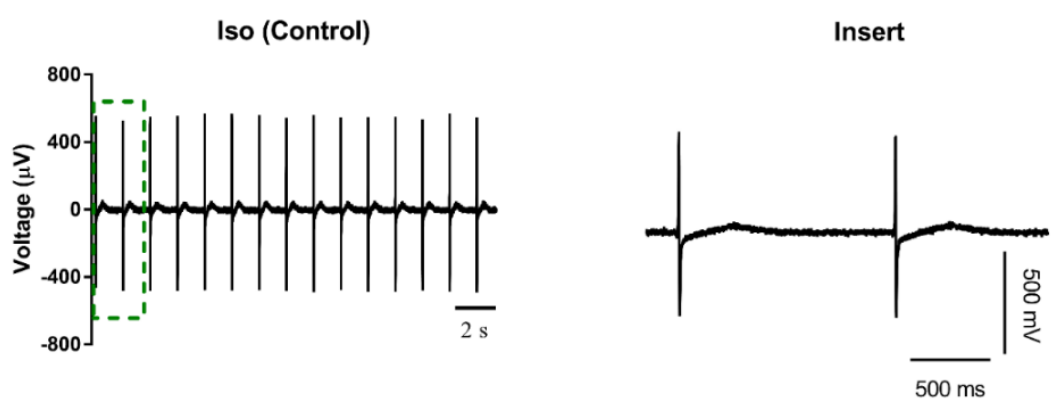

Iso (CPVT)

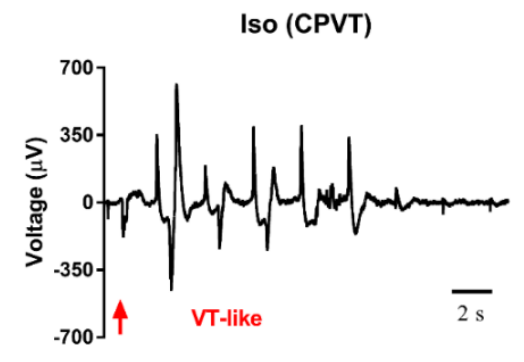

B
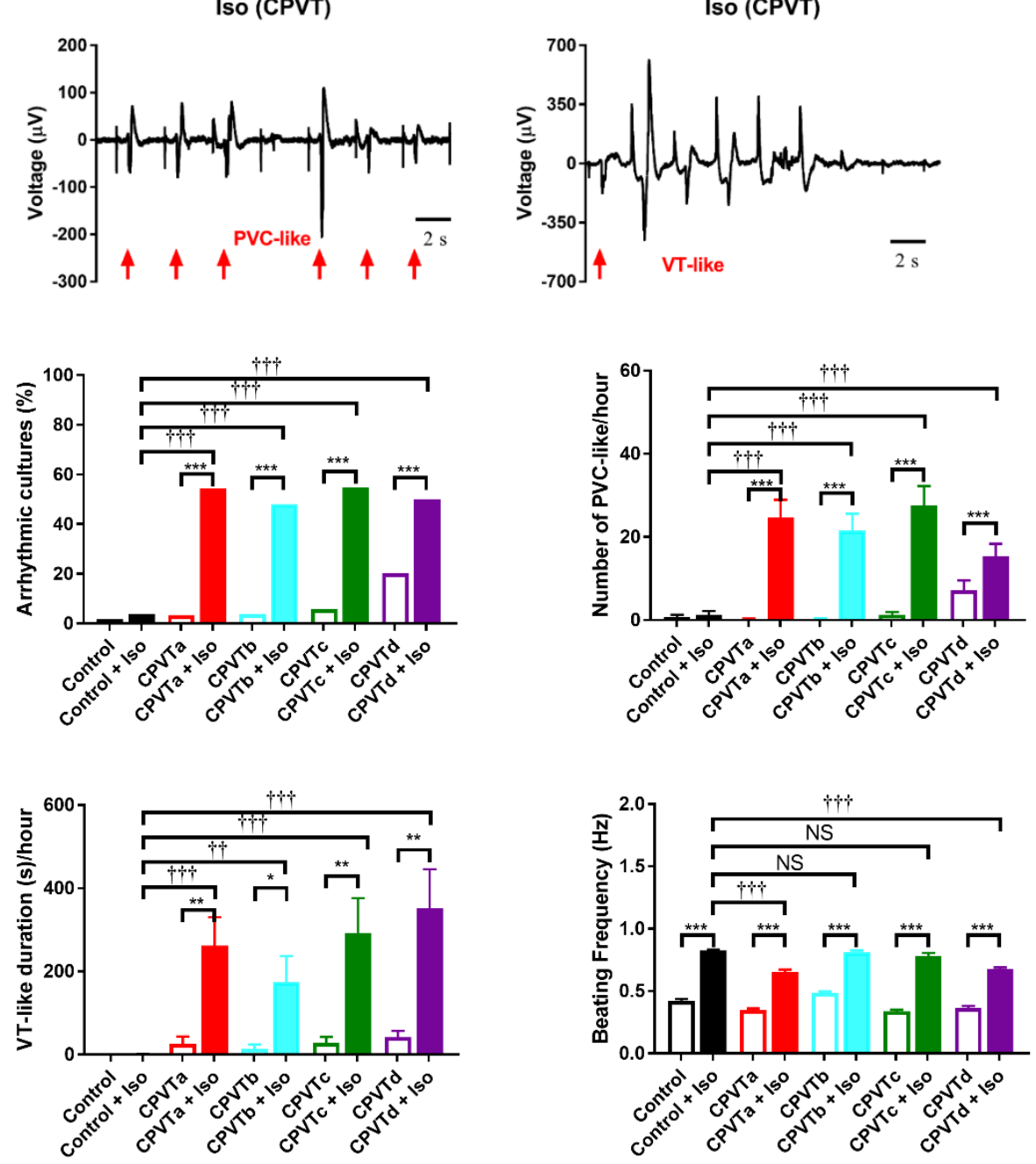

Figure 30. CPVT-CMs generated plenty of PVC-like and VT-like events after ISO stimulation. The MEA recording of ISO-treated Ctrl-CMs shows rhythmic beating. Representative traces with PVC-like and VT-like events in CPVT-CMs after ISO incubation for 15 minutes are shown (A). Compared to their corresponding basal condition, all the four parameters: arrhythmic cultures, the number of PVC-like events, duration of VT-like events, and beating frequency significantly increased in CPVT-CMs after ISO stimulation (B). Compared to Ctrl-CMs in ISO-treated condition, all the three 
parameters: arrhythmic cultures, number of PVC-like events, and duration of VT-like events significantly increased in CPVT-CMs after ISO stimulation. After ISO treatment, the beating frequencies were significantly lower in $\operatorname{CPVT}(\mathrm{a}, \mathrm{d})$-CMs compared with $\mathrm{Ctrl}-\mathrm{CMs}$ (B). For the ISOchallenged MEA experiments, culture numbers of Ctrl-CMs and CPVT(a, b, c, d)-CMs equal to 108, $92,77,104$, and 84 , respectively.

\subsection{Application of CPVT-CMs for drug testing}

\subsubsection{Antiarrhythmic effects of flecainide}

Flecainide is a class antiarrhythmic agent used to prevent and treat tachyarrhythmias. Watanabe et al. reported that flecainide prevented CPVT in mice and humans by decreasing RYR2 opening probability (Watanabe et al., 2009). Meanwhile, Liu N et al. reported that flecainide had an antiarrhythmic effect in a CPVT mouse model by increasing the threshold for triggered activity (through its sodium channel blocking effect) (Liu et al., 2011). Recently, Itzhaki et al. showed that flecainide completely eliminated or significantly decreased DADs and DAD-induced TAs in patient-specific CPVT (M4109R)-hiPSCs-CMs (Itzhaki et al., 2012). To test whether flecainide had different antiarrhythmic effects on $\operatorname{CPVT}(\mathrm{a}, \mathrm{b}, \mathrm{c}, \mathrm{d})$-CMs harboring different mutations (R420W, A2254V, E4076K, and H4742Y), two electrophysiological methods were applied to assess the arrhythmic events in $\operatorname{Ctrl-CMs~and~} \operatorname{CPVT}(a, b, c, d)-C M s$, which were challenged by $100 \mathrm{nM}$ ISO and then incubated with $1 \mu \mathrm{M}$ flecainide for one to two hours.

Patch-clamp analysis showed that the percentages of arrhythmic cells decreased significantly in CPVTa-CMs (22.58\%, n=31) and CPVTc-CMs $(46.43 \%, \mathrm{n}=28)$ after flecainide treatment, but not significantly in CPVTb-CMs $(69.23 \%, \mathrm{n}=13)$ and CPVTd-CMs $(64.29 \%, \mathrm{n}=14)$ compared to their respective ISO-challenged conditions (Figure 31A). The number of DADs and DADinduced TAs significantly decreased in CPVTa-CMs $(4.4 \pm 1.59, \mathrm{n}=31)$, CPVTb-CMs $(16.86 \pm$ 5.8, $\mathrm{n}=13)$, CPVTc-CMs (13.54 $\pm 3.69, \mathrm{n}=32)$, but not in CPVTd-CMs $(12.05 \pm 5.99, \mathrm{n}=14)$, when compared to their respective ISO-challenged conditions (Figure 31B). Interestingly, Number of EADs and EAD-induced TAs increased in CPVTd-CMs (91.35 $\pm 34.78, \mathrm{n}=14)$ after flecainide incubation (Figure 31). The beating frequencies were decreased in Ctrl-CMs and in CPVT(a, b, c, d)-CMs after flecainide incubation. 
A

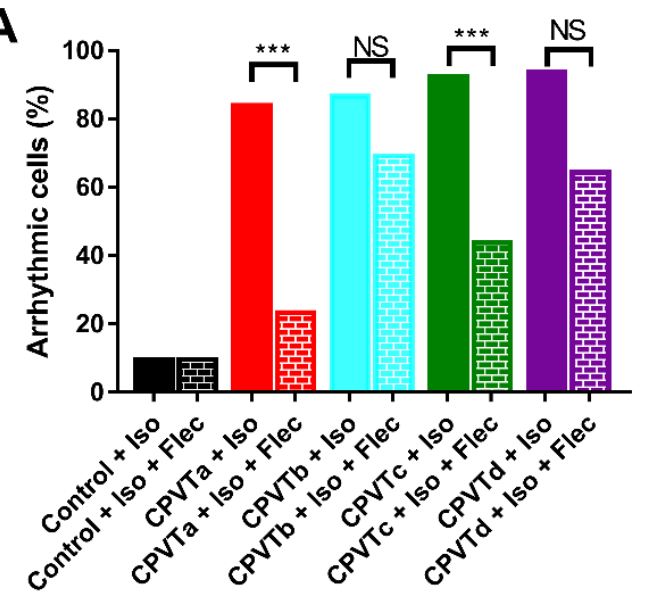

C

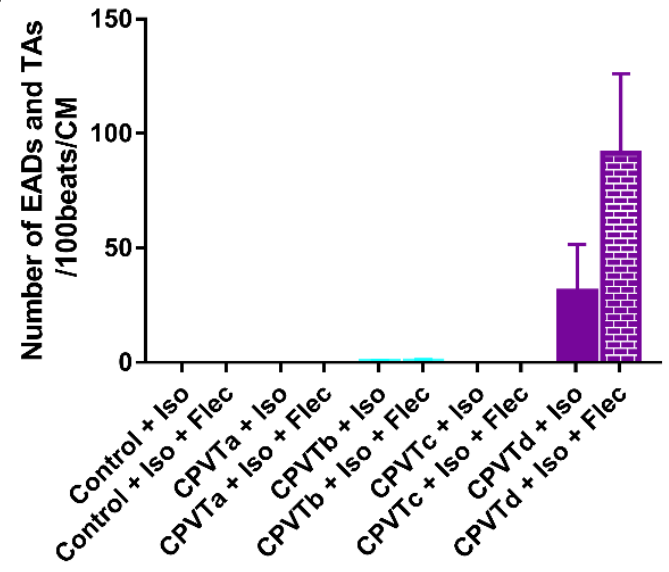

B

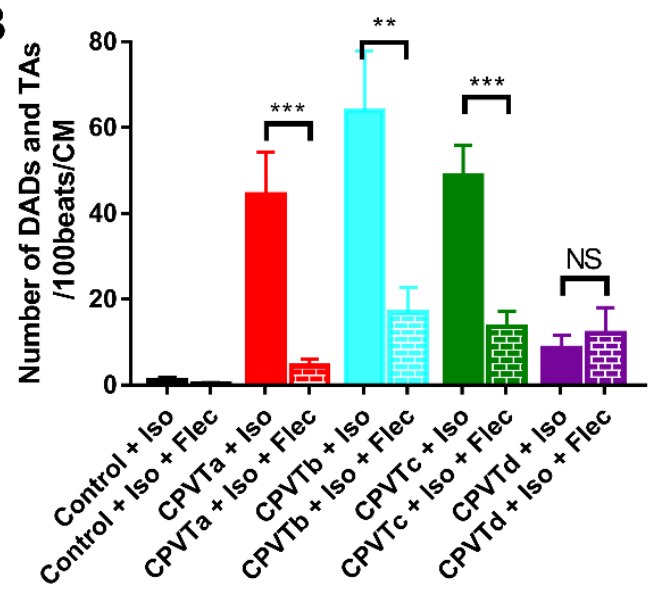

D

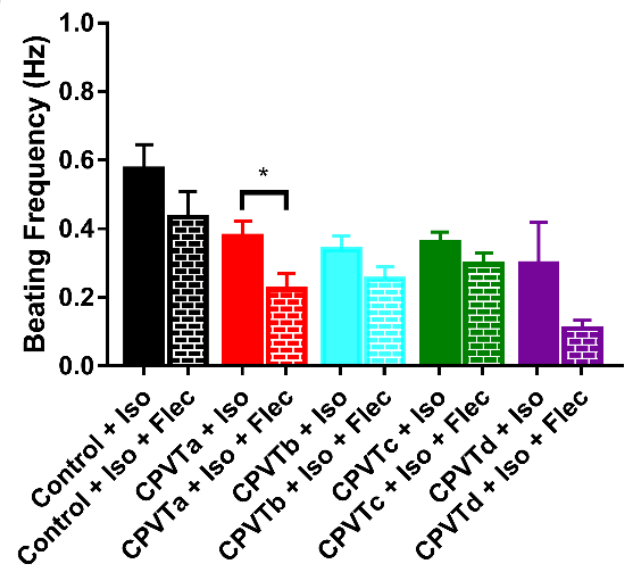

Figure 31. Systematic assessment of antiarrhythmic effects of flecainide by patch-clamp technique. (A) Summary of the percentage of CPVT- and Ctrl-CMs displaying arrhythmic beating in ISO-challenged condition and flecainide-treated condition. (B) Number of DADs and TAs significantly decreased in CPVT(a, b, c)-CMs but not in CPVTd-CMs after treated with flecainide. (C) Number of EADs and TAs increased in CPVTd-CMs after flecainide incubation. (D) The beating frequencies in all Ctrl-CMs and CPVT(a, b, c, d)-CMs decreased after flecainide treatment. For the flecainide-treated patch-clamp experiments, cell numbers of Ctrl-CMs and CPVT(a, b, c, d)-CMs equal to 21, 31, 13, 28 and 14, respectively.

MEA recordings showed the significantly decreased percentages of arrhythmic cultures after flecainide treatment in CPVTa-CMs (4.1\%), CPVTb-CMs (0\%), CPVTc-CMs (6.6\%) but not in CPVTd-CMs (16.6\%), when compared to respective ISO-challenged CPVTa-CMs (58.3\%), CPVTb-CMs (54.1\%), CPVTc-CMs (63.3\%), and CPVTd-CMs (44.4\%) (Figure 32A). The number of PVC-like events significantly decreased in CPVTa-CMs $(1.639 \pm 1.639)$, CPVTb- 
CMs $(0 \pm 0)$, CPVTc-CMs $(1.28 \pm 0.89)$, and CPVTd-CMs $(2.56 \pm 1.78)$ after flecainide treatment, when compared to respective ISO-challenged CPVTa-CMs (25.75 \pm 5.54$)$, CPVTbCMs (19.12 \pm 5.98$)$, CPVTc-CMs $(41.9 \pm 12.22)$, and CPVT-d-CMs (18.93 \pm 7.26$)$ (Figure 32B).

Moreover, the VT-like events in MEA recording disappeared in $\operatorname{CPVT}(\mathrm{a}, \mathrm{b}, \mathrm{c})$-CMs but not in CPVTd-CMs (95.91 $\pm 95.91 \mathrm{~s} /$ hour) after flecainide incubation (Figure 32C). In addition, the flecainide treatment resulted in significantly reduced beating frequencies of Ctrl-CMs and CPVT(a, b, c, d)-CMs (Figure 32D).

These results indicate that flecainide had antiarrhythmic effects on CPVT(a, b, c)-CMs, but minor effect on CPVTd-CMs. 
A

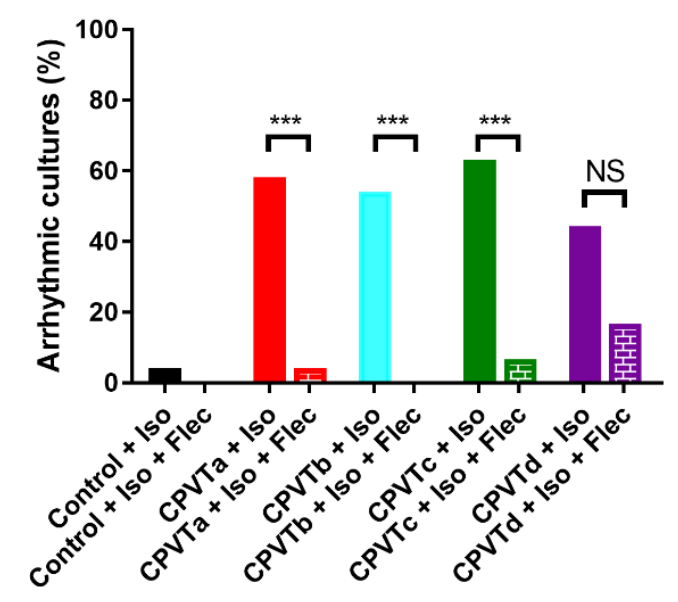

C

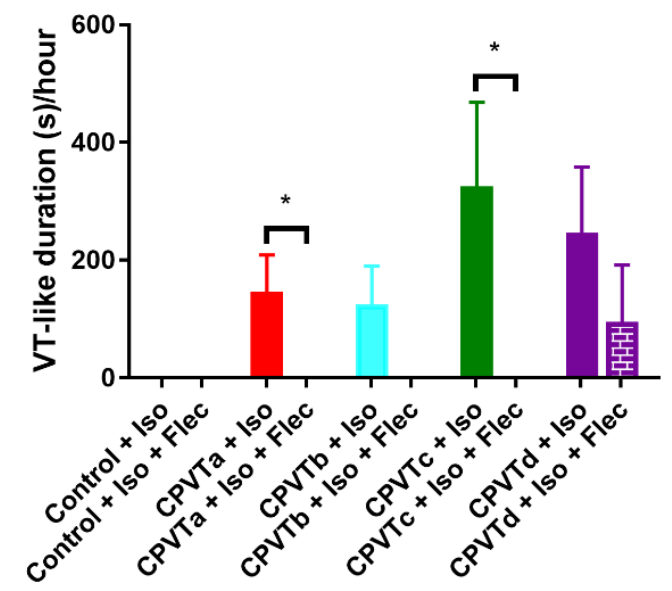

B

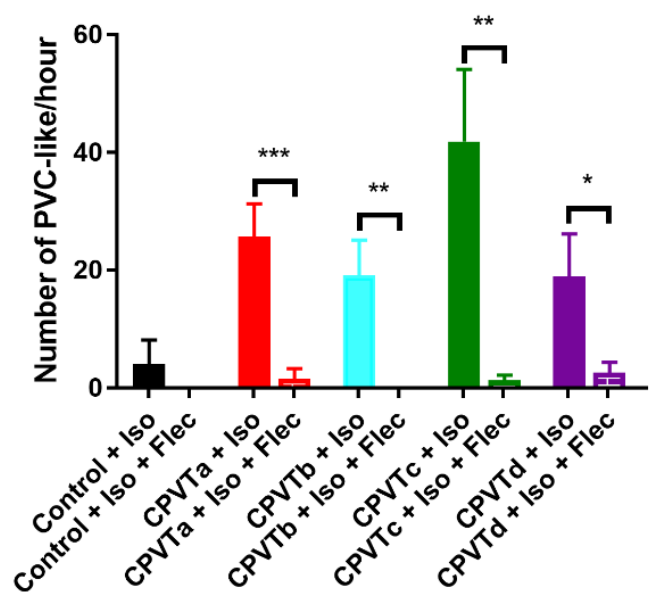

D

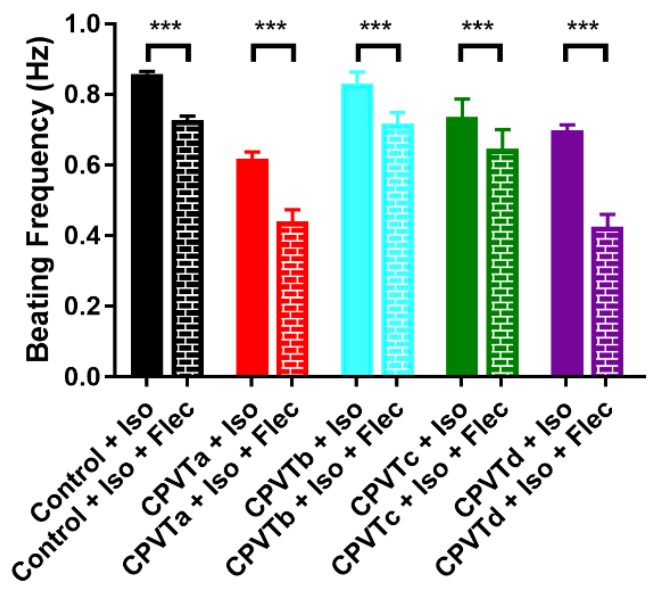

Figure 32. MEA data confirmed antiarrhythmic effects of flecainide for CPVT(a, b, c)-CMs. (A) Percentage of cultures in Ctrl-CMs and CPVT-CMs which displayed PVC-like and VT-like events. Notably, flecainide reduced the arrhythmic cultures in CPVT(a, b, c)-CMs but not in CPVTd-CMs when compared to the cells only challenged with ISO, respectively. (B) Number of PVC-like events significantly decreased in all CPVT-CMs after flecainide treatment. (C) VT-like events disappeared in CPVT(a, b, c)-CMs but not in CPVTd-CMs after flecainide treatment. (D) Beating frequencies decreased significantly in both Ctrl-CMs and CPVT-CMs. For the flecainide-treated MEA experiments, culture numbers of Ctrl-CMs and CPVT(a, b, c, d)-CMs equal to 24, 24, 24, 30 and 18, respectively.

\subsubsection{Antiarrhythmic effects of dantrolene}

Dantrolene is a specific agent for the treatment of malignant hyperthermia. There were several publications demonstrating that dantrolene has antiarrhythmic effects on CPVT (Jung et al., 2012; Penttinen et al., 2015). To test whether dantrolene has different antiarrhythmic effects on 
CPVT(a, b, c, d)-CMs harboring different mutations (R420W, A2254V, E4076K and H4742Y), two electrophysiological methods (Figure 33, Figure 34) were used to assess the arrhythmic events of Ctrl-CMs and CPVT(a, b, c, d)-CMs, which were challenged by $100 \mathrm{nM}$ ISO and then incubated with $1 \mu \mathrm{M}$ dantrolene for one to two hours.

Patch-clamp analysis showed that the percentage of arrhythmic cells decreased significantly in CPVTa-CMs $(20.7 \%, \mathrm{n}=29)$ and CPVTc-CMs $(31 \%, \mathrm{n}=32)$ after dantrolene treatment, but not in CPVTb-CMs $(70 \%, n=10)$ and CPVTd-CMs $(84.6 \%, n=13)$, compared to their respective ISOchallenged conditions (Figure 33A). The number of DADs and DAD-induced TAs significantly decreased in CPVTa-CMs (2.9 $\pm 1.2, n=29)$, CPVTb-CMs (11.28 $\pm 6, n=10)$, CPVTc-CMs (4.07 $\pm 1.4, \mathrm{n}=32)$, but increased in CPVTd-CMs $(11.98 \pm 3.9, \mathrm{n}=13)$, compared to their respective ISO-challenged conditions (Figure 33B). Interestingly, Number of EADs and TAs increased in CPVTd-CMs $(49.87 \pm 23.59, \mathrm{n}=13)$ after dantrolene incubation (Figure 33C). The beating frequency of CPVTc-CMs $(0.51 \pm 0.06 \mathrm{~Hz})$ significantly increased after dantrolene treatment (Figure 33D). 
A
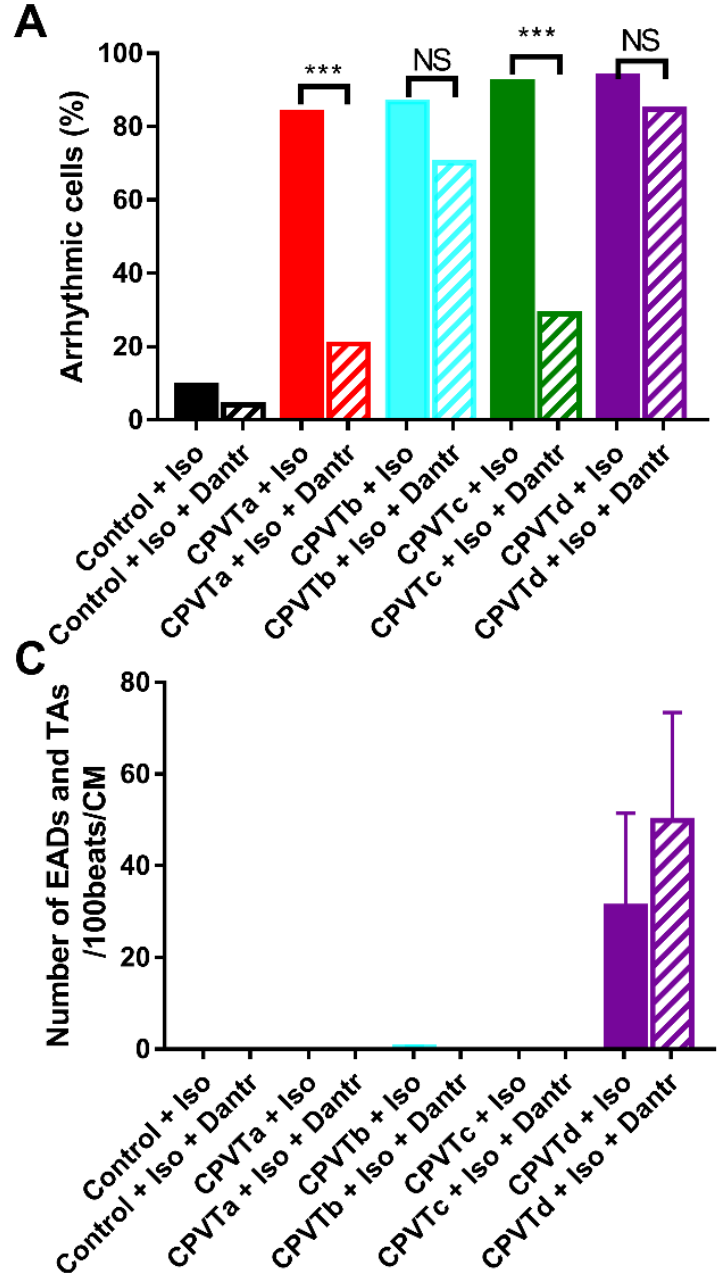

B

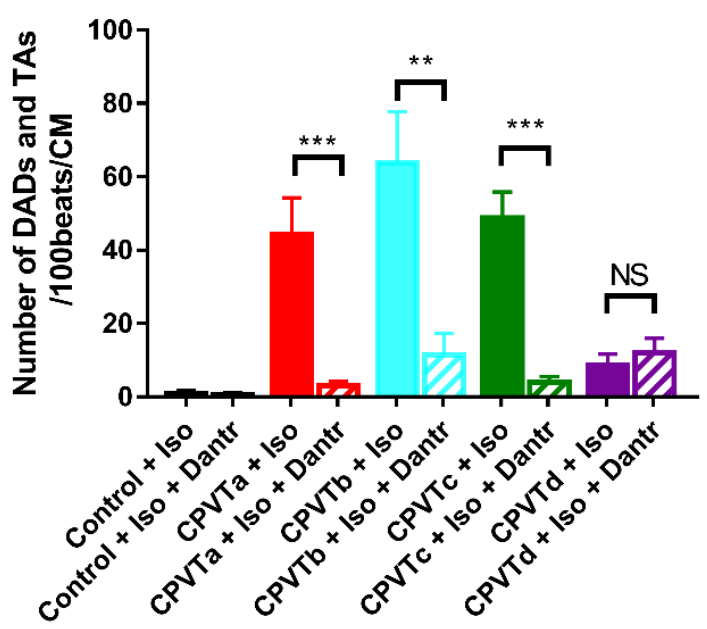

D

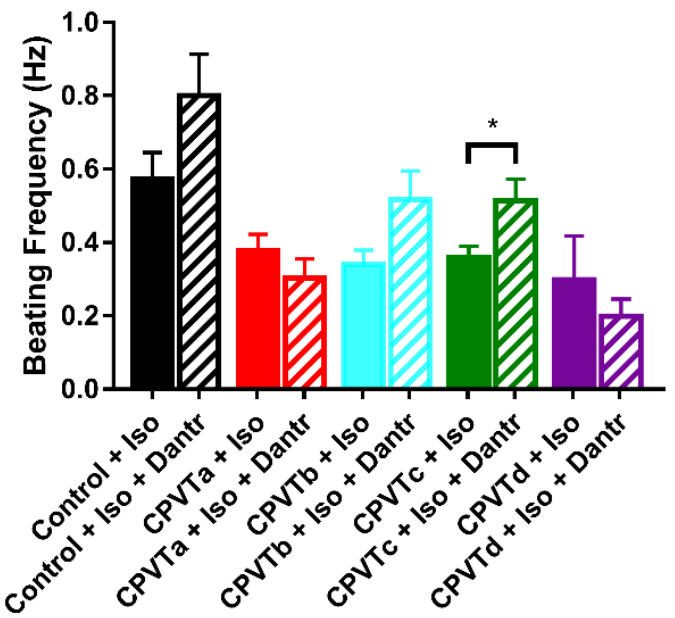

Figure 33. Systematic assessment of antiarrhythmic effects of dantrolene by patch-clamp technique. (A) Summary of the percentage of CPVT-CMs and Ctrl-CMs displaying arrhythmic beating in ISOchallenged condition and dantrolene-treated condition. (B) Number of DADs and TAs significantly decreased in CPVT(a, b, c)-CMs but not in CPVTd-CMs after treated with dantrolene. (C) Number of EADs and TAs increased in CPVTd-CMs after dantrolene incubation. (D) The beating frequencies in CPVTc-CMs increased significantly after dantrolene treatment. For the dantrolene-treated patch-clamp experiments, cell numbers of Ctrl-CMs and CPVT(a, b, c, d)-CMs equal to and 24, 29, 10, 32 and 13, respectively. 
MEA recordings showed the significantly decreased percentages of arrhythmic cultures after flecainide treatment in CPVTa-CMs (4.2\%), CPVTb-CMs (0\%), CPVTc-CMs (0\%) and CPVTdCMs (20.1\%), when compared to respective ISO-challenged CPVTa-CMs (50\%), CPVTb-CMs (38.9\%), CPVTc-CMs (53.6\%), and CPVTd-CMs (54.2\%) (Figure 34A). The number of PVClike events significantly decreased in CPVTa-CMs (0.7 \pm 0.7$)$, CPVTb-CMs $(0 \pm 0)$, CPVTcCMs $(0 \pm 0)$, and CPVTd-CMs $(5.6 \pm 2.9)$ after flecainide treatment, when compared to respective ISO-challenged CPVTa-CMs (35.3 \pm 12.6), CPVTb-CMs (18.26 \pm 7.4), CPVTc-CMs $(16.71 \pm 4.6)$, and CPVT-d-CMs (10.88 \pm 4.9$)$ (Figure 34B).

Moreover, the VT-like events in MEA recording disappeared in $\operatorname{CPVT}(\mathrm{a}, \mathrm{b}, \mathrm{c})$-CMs but not in CPVTd-CMs (150 \pm 150 s/hour) after dantrolene incubation (Figure 34C). In addition, the flecainide treatment resulted in significantly reduced beating frequencies in Ctrl-CMs and CPVT(a, b, d)-CMs (Figure 34D).

These results indicate that dantrolene had antiarrhythmic effects on CPVT(a, b, c)-CMs but minor effect on CPVTd-CMs. 
A

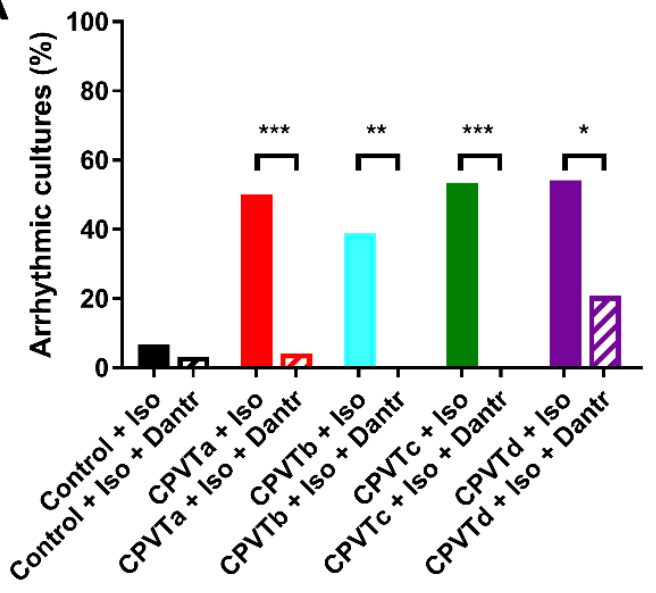

C

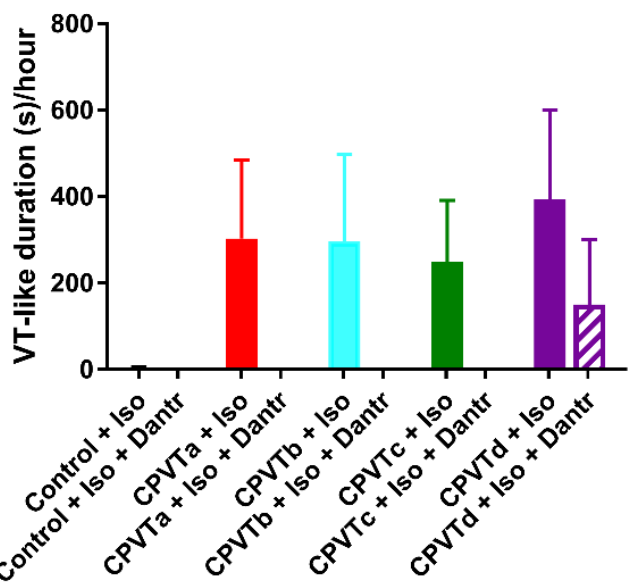

B

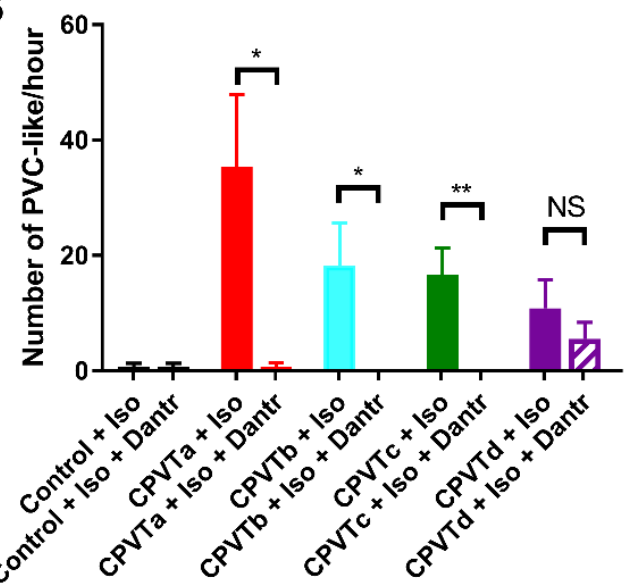

D

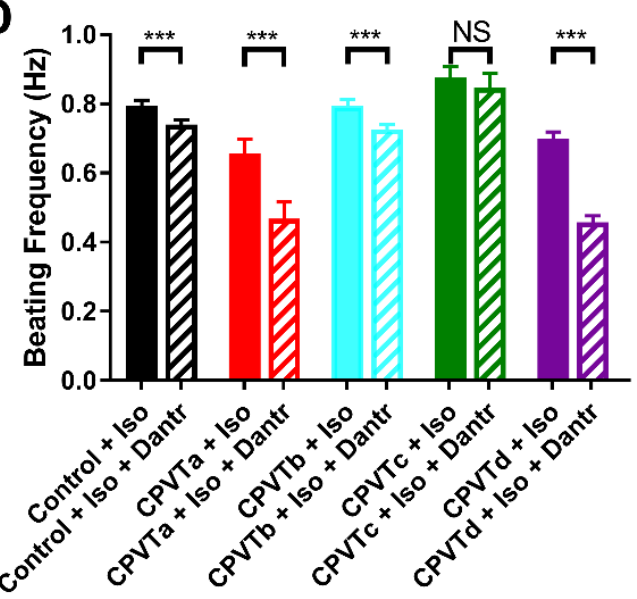

Figure 34. MEA data confirmed antiarrhythmic effects of dantrolene for CPVT(a, b, c)-CMs. (A) Percentage of cultures in Ctrl-CMs and CPVT-CMs which displayed PVC-like and VT-like events. Dantrolene reduced the arrhythmic cultures in $\operatorname{CPVT}(\mathrm{a}, \mathrm{b}, \mathrm{c}, \mathrm{d})-\mathrm{CMs}$ when compared to the cells only challenged with ISO, respectively. (B) Number of PVC-like events significantly decreased in CPVT(a, b, c)-CMs, but not in CPVTd-CMs after dantrolene treatment. (C) VT-like events disappeared in CPVT(a, b, c)-CMs, but not in CPVTd-CMs after dantrolene treatment. (D) Beating frequencies decreased significantly in Ctrl-CMs and CPVT(a, b, d)-CMs, but not in CPVTc-CMs when compared with ISOchallenged condition, respectively. For the dantrolene-treated MEA experiments, culture numbers of CtrlCMs and CPVT(a, b, c, d)-CMs equal to 30, 24, 18, 28 and 24, respectively.

\subsubsection{Antiarrhythmic effects of rycal1}

RYR channel stabilizers (rycals) restore the normal function of RYR channels and are not RYR2 channel blockers. Two CPVT treatment drugs rycal1 and rycal2 were provided by a 
pharmacological company for this study. To test whether rycall has antiarrhythmic effects on CPVT(a, b, c, d)-CMs harboring different mutations (R420W, A2254V, E4076K and H4742Y), two electrophysiological methods (Figure 35, Figure 36) were applied to assess the arrhythmic events of Ctrl-CMs and CPVT(a, b, c, d)-CMs, which were challenged by $100 \mathrm{nM}$ ISO and then incubated with $10 \mu \mathrm{M}$ rycall for one to two hours.

Patch clamp analysis showed that the percentages of arrhythmic cells decreased significantly in CPVTa-CMs (25\%, n=32), CPVTb-CMs (41.7\%, n=12), CPVTc-CMs (32.4\%, n=37) and CPVTd-CMs $(60 \%, \mathrm{n}=15)$ after rycal1 treatment, when compared to their respective ISOchallenged conditions (Figure 35A). The number of DADs and DAD-induced TAs significantly decreased in CPVTa-CMs (5.7 $\pm 2, n=32)$, CPVTb-CMs (10.3 $\pm 5.4, n=12)$, CPVTc-CMs $(5.1 \pm$ 1.8, $\mathrm{n}=37)$, but increased in CPVTd-CMs $(13.6 \pm 5.8, \mathrm{n}=15)$, when compared to their respective ISO-challenged conditions (Figure 35B). Interestingly, Number of EADs and EAD-induced TAs increased in CPVTd-CMs $(56.3 \pm 32.6, \mathrm{n}=15)$ after rycall incubation (Figure 35C). The beating frequencies decreased significantly in CPVTa-CMs after rycall treatment, increased but not significantly in CPVTb-CMs, and showed no changes in Ctrl- and CPVT(c, d)-CMs (Figure 35D). 
A
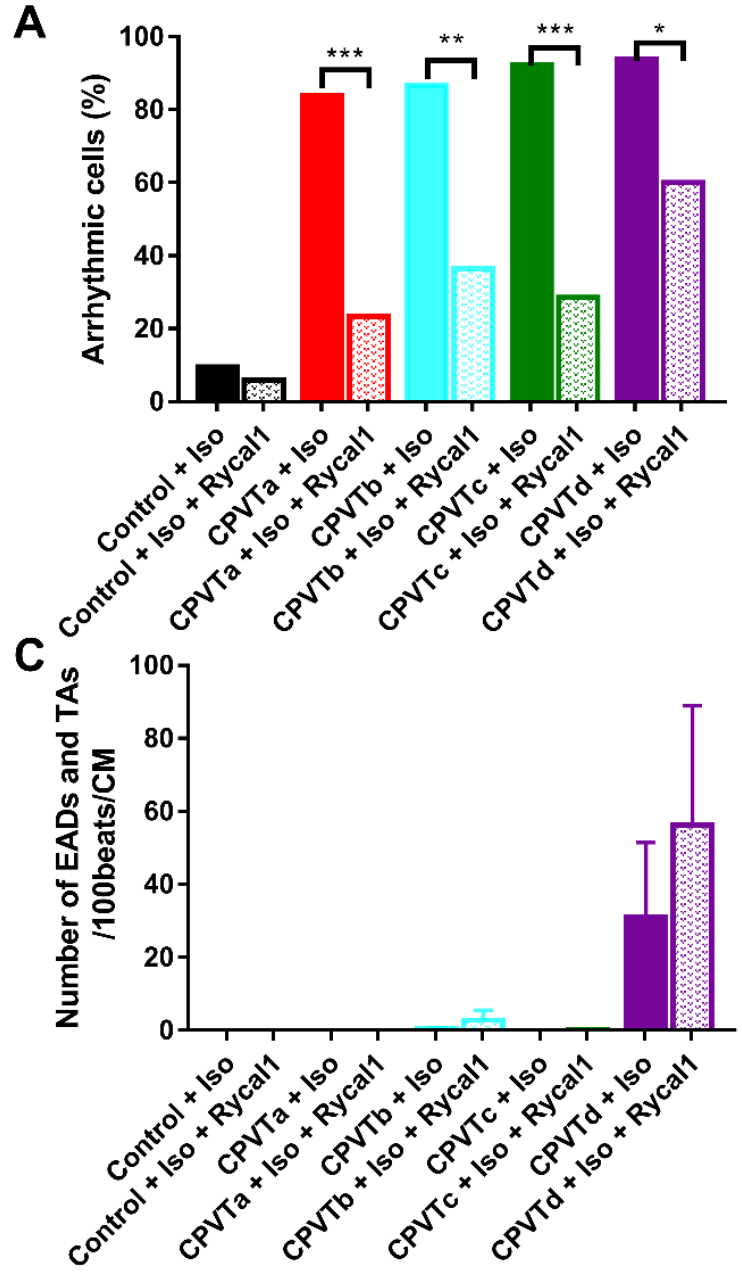

B

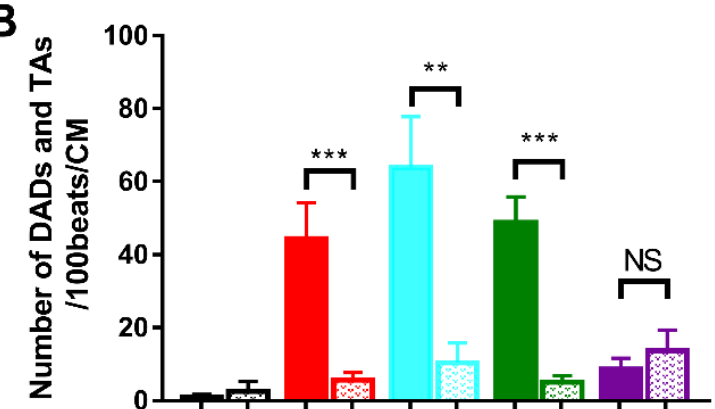

D
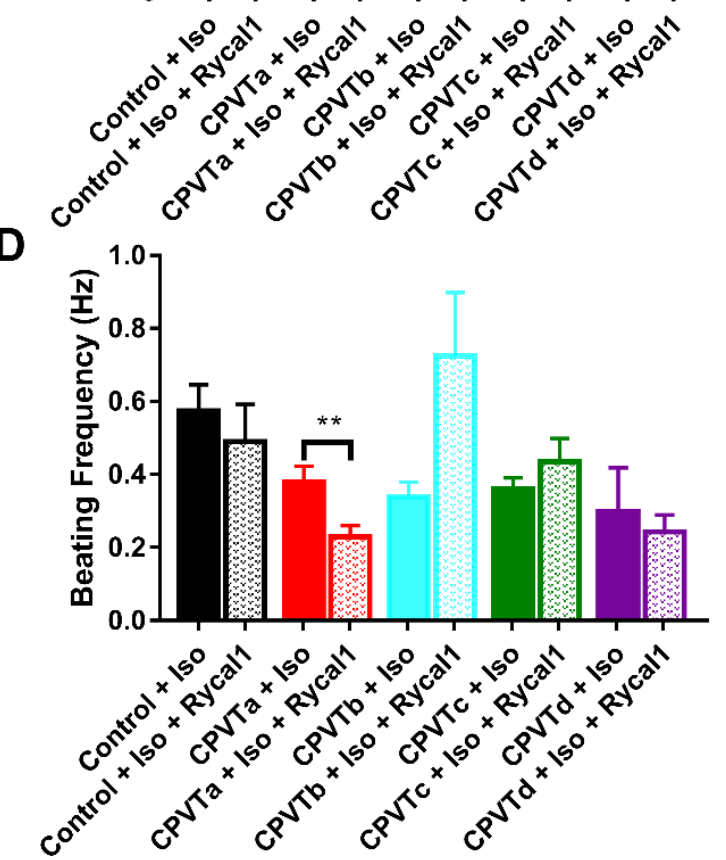

Figure 35. Systematic assessment of antiarrhythmic effects of rycal1 by patch-clamp technique. (A) Summary of the percentage of CPVT- and Ctrl-CMs displaying arrhythmic beating in ISO-challenged condition and flecainide-treated condition. (B) Number of DADs and TAs significantly decreased in CPVT(a, b, c)-CMs but increased in CPVTd-CMs after treated with rycall. (C) Number of EADs and TAs increased in CPVTd-CMs after rycall incubation. (D) The beating frequencies in CPVTa-CMs decreased significantly after rycal1 treatment. For the rycal1-treated patch-clamp experiments, cell numbers of Ctrl-CMs and CPVT(a, b, c, d)-CMs equal to 17, 32, 12, 37 and 15, respectively.

MEA recordings showed the significantly decreased percentages of arrhythmic cultures after rycal1 treatment in CPVTa-CMs (0\%) and CPVTb-CMs (0\%), but not in CPVTc-CMs (5.6\%) and CPVTd-CMs (33.3\%), when compared to respective ISO-challenged CPVTa-CMs (66.7\%), CPVTb-CMs (41.7\%), CPVTc-CMs (33.3\%), and CPVTd-CMs (61.1\%) (Figure 36A). The number of PVC-like events significantly decreased in CPVTa-CMs $(0 \pm 0)$ and CPVTc-CMs $(0$ $\pm 0)$ but not in CPVTb-CMs $(0 \pm 0)$ and CPVTd-CMs $(10.17 \pm 6.4)$ after rycal1 treatment, when 
compared to respective ISO-challenged CPVTa-CMs (16.95 \pm 4.1$)$, CPVTb-CMs $(28 \pm 15.77)$, CPVTc-CMs (25.42 \pm 10.66), and CPVT-d-CMs (23.23 \pm 8.4) (Figure 36B).

Moreover, the VT-like events in MEA recording disappeared in CPVT(a, b)-CMs, but not in CPVTc-CMs (50 $\pm 50 \mathrm{~s} /$ hour $)$ and CPVTd-CMs $(216 \pm 122.3 \mathrm{~s} /$ hour $)$ after rycall incubation (Figure 36C). In addition, the rycall treatment resulted in significantly reduced beating frequencies in Ctrl-CMs and CPVT(a, b, d)-CMs, but not in CPVTc-CMs (Figure 36D).

These results indicated that rycal1 had antiarrhythmic effects on $\operatorname{CPVT}(a, b, c)-C M s$ but minor effect on CPVTd-CMs. 
A

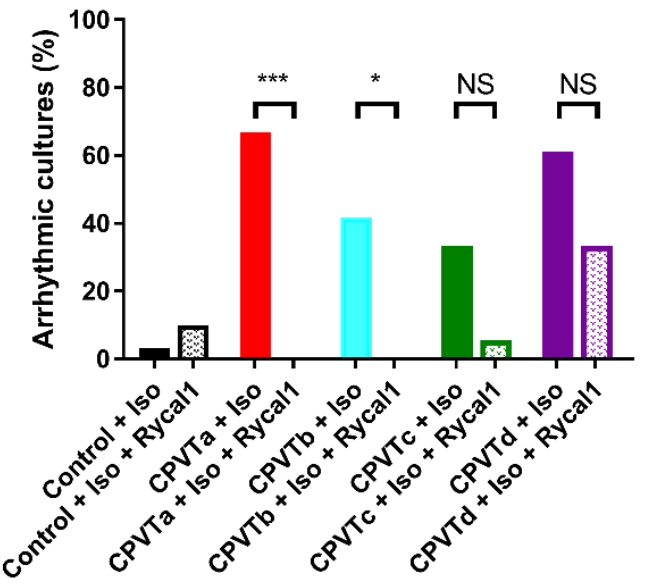

C

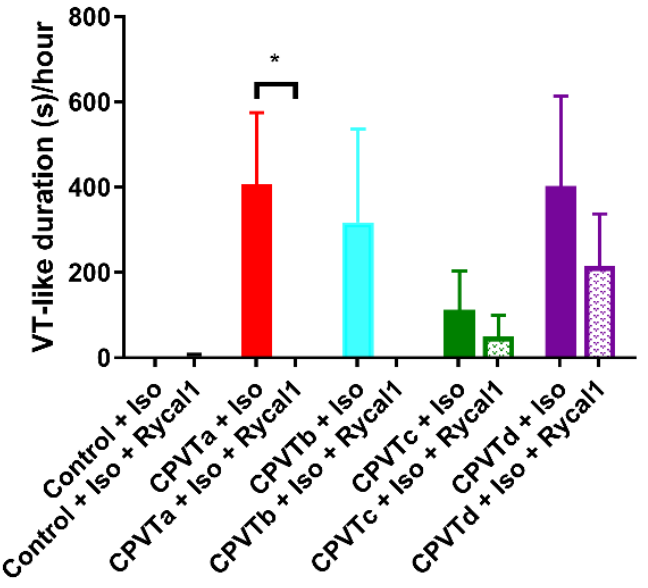

B

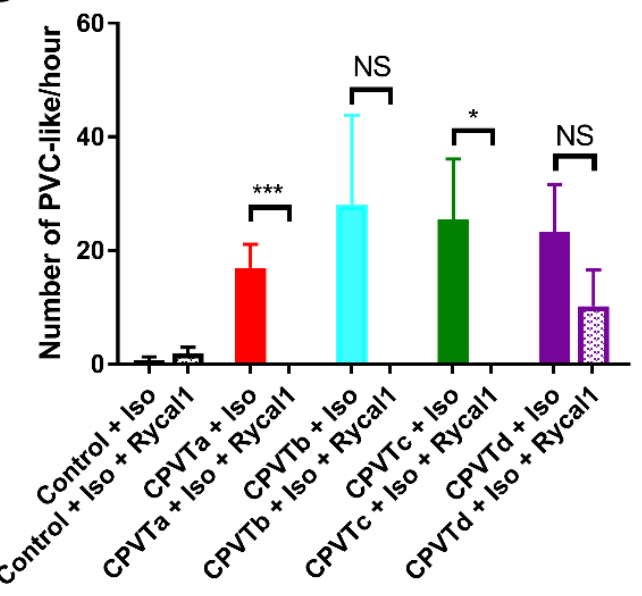

D

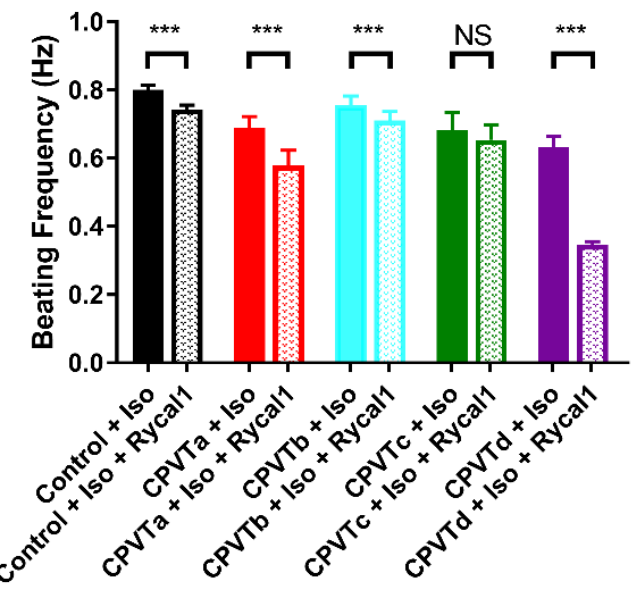

Figure 36. MEA data confirmed antiarrhythmic effects of rycal1 for CPVT(a, b, c)-CMs. (A) Rycal1 reduced the arrhythmic cultures (displaying PVC-like and VT-like events) in CPVT(a, b)-CMs when compared to the cells only challenged with ISO, respectively. (B) Number of PVC-like events significantly decreased in CPVT(a, c)-CMs, but not in CPVT(b, d)-CMs after rycal1 treatment. (C) VTlike events disappeared in $\operatorname{CPVT}(\mathrm{a}, \mathrm{b})-\mathrm{CMs}$, but not in $\operatorname{CPVT}(\mathrm{c}, \mathrm{d})-\mathrm{CMs}$ after rycal1 treatment. (D) Beating frequencies decreased significantly in Ctrl-CMs and CPVT(a, b, d)-CMs, but not in CPVTc-CMs when compared with their ISO-challenged condition, respectively. For the rycal1-treated MEA experiments, culture numbers of Ctrl-CMs and CPVT(a, b, c, d)-CMs equal to 30, 24, 12, 18 and 18, respectively.

\subsubsection{Antiarrhythmic effects of rycal2}

To test whether rycal2 has different antiarrhythmic effects on CPVT(a, b, c, d)-CMs harboring different mutations (R420W, A2254V, E4076K and H4742Y), two electrophysiological methods (Figure 37, Figure 38) were applied to assess the arrhythmic events of Ctrl-CMs and CPVT(a, b, 
c, d)-CMs, which were challenged with $100 \mathrm{nM}$ ISO and then incubated with $10 \mu \mathrm{M}$ rycal2 for one to two hours.

Patch clamp analysis showed that the percentages of arrhythmic cells decreased significantly in CPVTa-CMs (29.6\%, n=27), CPVTb-CMs (57.1\%, $\mathrm{n}=14)$ and CPVTc-CMs (38.9\%, $\mathrm{n}=36)$ after rycal2 treatment, but not significantly in CPVTd-CMs $(64.7 \%, \mathrm{n}=17)$ compared to their respective ISO-challenged conditions (Figure 37A). The number of DADs and DAD-induced TAs significantly decreased in CPVTa-CMs (5.2 $\pm 2, n=27)$, CPVTb-CMs (5.2 $\pm 2.3, n=14)$, CPVTc-CMs (4.9 $\pm 1.8, \mathrm{n}=36)$, but not in CPVTd-CMs $(9.3 \pm 3.3, \mathrm{n}=17)$, when compared to their respective ISO-challenged conditions (Figure 37B). Interestingly, Number of EADs and EAD-induced TAs decreased slightly but not significantly in CPVTd-CMs (13.67 $\pm 7.9, \mathrm{n}=17)$ after rycal2 incubation (Figure 37C). Notably, there were some EADs and TAs in CPVTb-CMs and CPVTc-CMs after rycal2 incubation (Figure 37C). The beating frequencies were significantly decreased in CPVTa-CMs $(0.25 \pm 0.05 \mathrm{~Hz})$ and CPVTb-CMs $(0.15 \pm 0.04 \mathrm{~Hz})$ after rycal2 incubation (Figure 37D). 

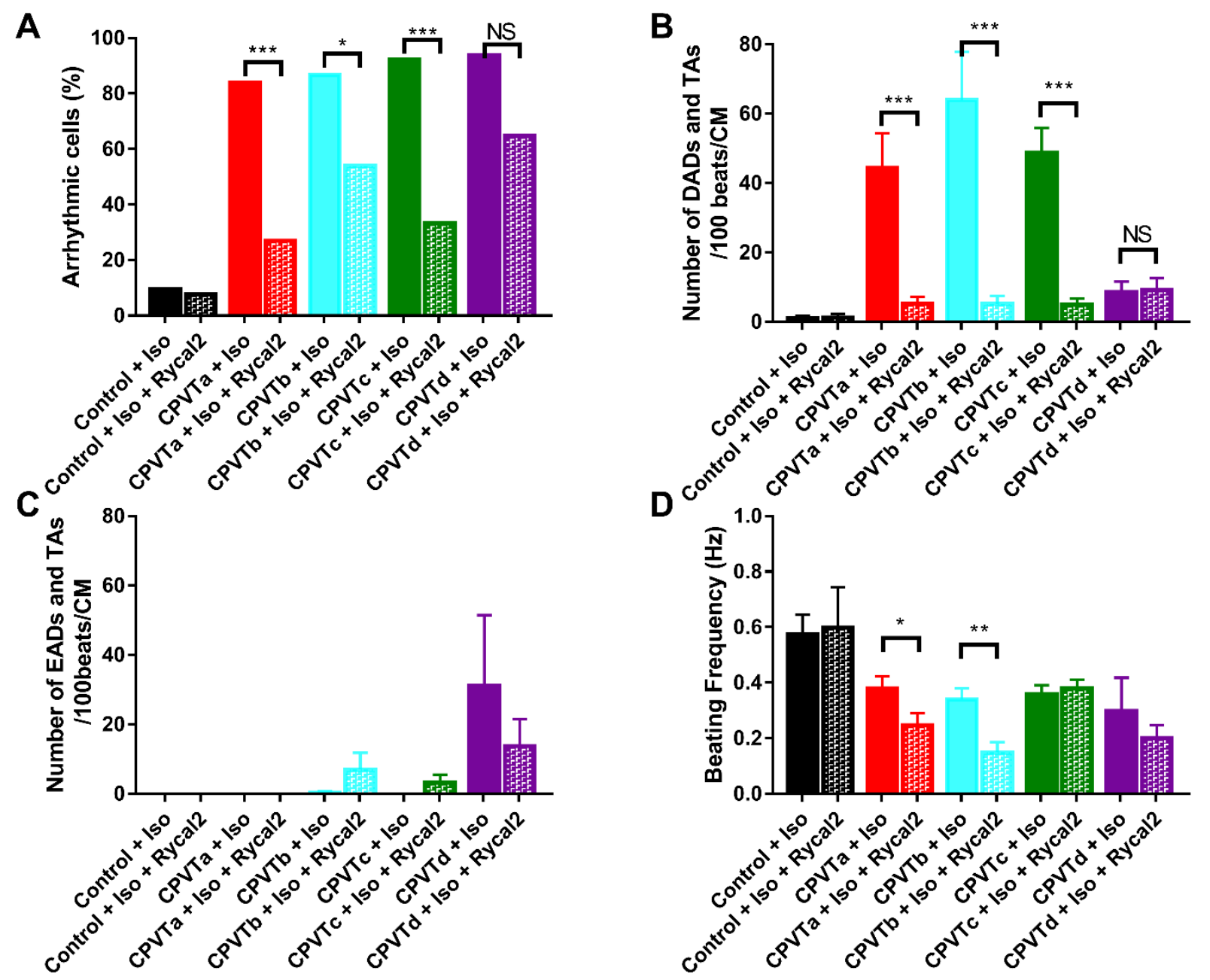

Figure 37. Systematic assessment of antiarrhythmic effects of rycal2 by patch-clamp technique. (A) Summary of the percentage of CPVT- and Ctrl-CMs displaying arrhythmic beating in ISO-challenged condition and flecainide-treated condition. (B) Number of DADs and TAs significantly decreased in CPVT(a, b, c)-CMs but not in CPVTd-CMs after treated with rycal2. (C) There were some EADs and TAs in CPVTb-CMs and CPVTc-CMs after rycal2 incubation. (D) The beating frequencies in CPVT(b, c)-CMs decreased significantly after rycal2 treatment. For the rycal2-treated patch-clamp experiments, cell numbers of Ctrl-CMs and CPVT(a, b, c, d)-CMs equal to 13, 27, 14, 36 and 17, respectively.

MEA recordings showed the significantly decreased percentages of arrhythmic cultures after rycal2 treatment in CPVTa-CMs (5\%), CPVTb-CMs (0\%), CPVTc-CMs (3.6\%) but not in CPVTd-CMs (29.2\%), when compared to respective ISO-challenged CPVTa-CMs (40\%), CPVTb-CMs (52.2\%), CPVTc-CMs (60.7\%), and CPVTd-CMs (41.7\%) (Figure 38A). The number of PVC-like events significantly decreased in CPVTa-CMs (0.94 \pm 0.94$)$, CPVTb-CMs 
(0 \pm 0$)$, CPVTc-CMs $(0.68 \pm 0.68)$ but not in CPVTd-CMs $(2.19 \pm 1.6)$ after rycal2 treatment, when compared to respective ISO-challenged CPVTa-CMs (19.83 \pm 9.4), CPVTb-CMs (23.51 \pm 7), CPVTc-CMs (24.34 \pm 8.19$)$, and CPVT-d-CMs (11.25 \pm 4.5$)$ (Figure 38B).

Moreover, the VT-like events in MEA recording disappeared in CPVT(a, b, c)-CMs, but increased in CPVTd-CMs (437.5 \pm 224.6 s/hour) after rycal2 incubation (Figure 38C). In addition, the rycal2 treatment resulted in significantly reduced beating frequencies in Ctrl-CMs and CPVT(a, b, d)-CMs, but significantly increased in CPVTc-CMs $(0.9 \pm 0.04 \mathrm{~Hz})$ (Figure 38D).

These results indicated that rycal2 had antiarrhythmic effects on CPVT(a, b, c)-CMs but minor effect on CPVTd-CMs. 
A
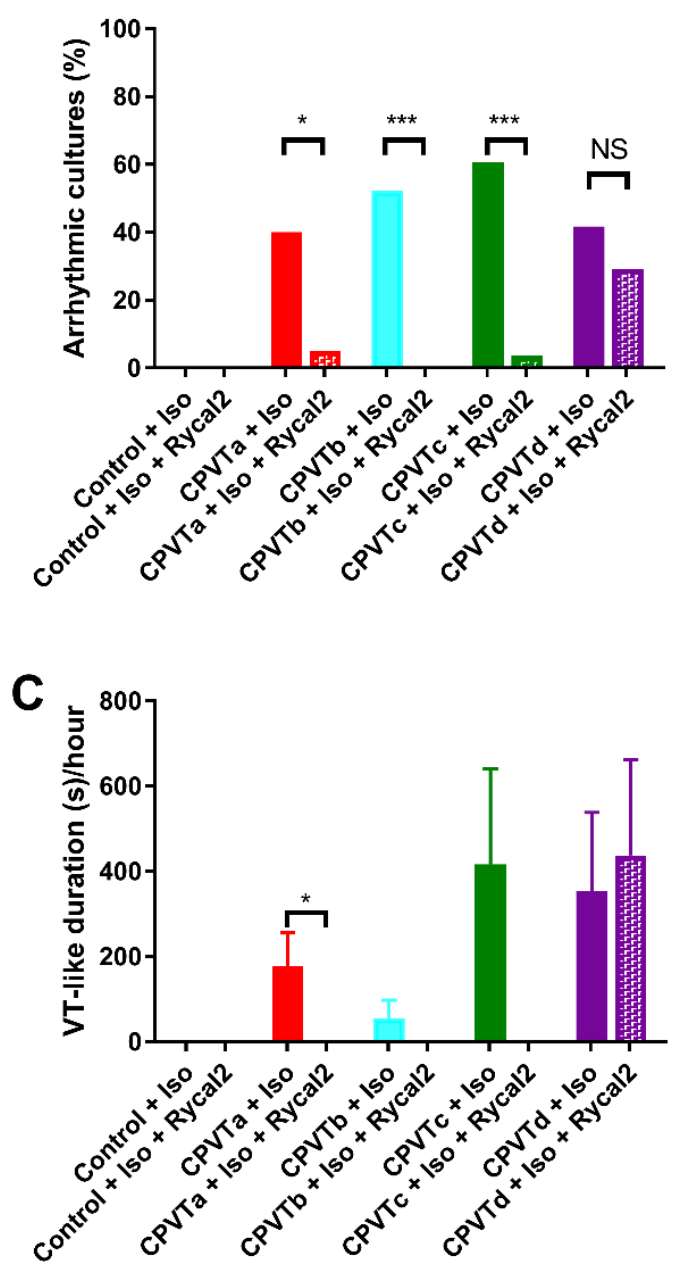

B
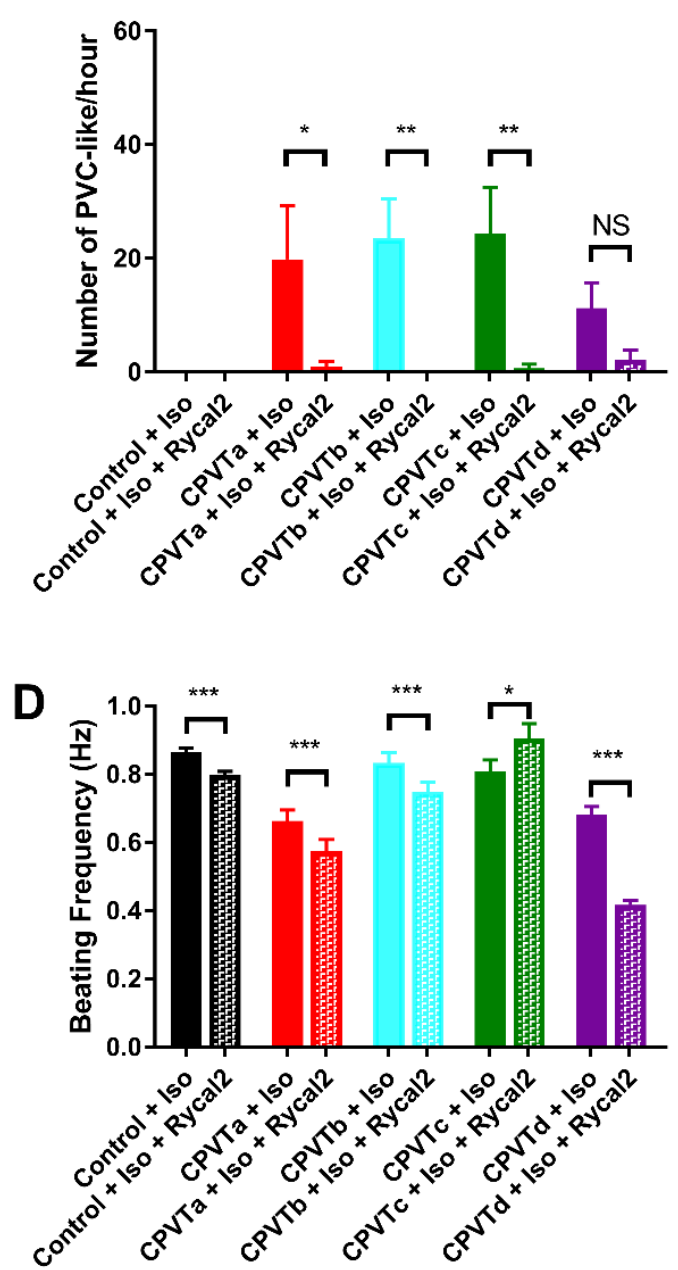

Figure 38. MEA data confirmed antiarrhythmic effects of rycal2 for CPVT(a, b, c)-CMs. (A) Rycal2 significantly reduced the arrhythmic cultures (displaying PVC-like and VT-like events) in CPVT(a, b, c)CMs, but not in CPVTd-CMs when compared to the cultures only challenged with ISO, respectively. (B) Number of PVC-like events significantly decreased in CPVT(a, b, c)-CMs but not in CPVTd-CMs after rycal2 treatment. (C) VT-like events disappeared in CPVT(a, b, c)-CMs, but increased in CPVTd-CMs after rycal2 treatment. (D) Beating frequencies decreased significantly in Ctrl-CMs and CPVT(a, b, d)$\mathrm{CMs}$, but increased significantly in CPVTc-CMs when compared to ISO-challenged condition, respectively. For the rycal2-treated MEA experiments, culture numbers of Ctrl-CMs and CPVT(a, b, c, d)-CMs equal to 24, 20,23, 28 and 24, respectively.

\section{4 cAMP dynamics in Epac1-camps- and Epac1-camps-JNC-transfected Ctrl- and CPVT-CMs}


$\beta$-adrenergic signaling pathway plays a central role in regulating the chronotropic, inotropic and lusitropic effects, and initiating CPVT. Previously published data demonstrated that the cAMPdegrading enzyme PDEs were upregulated or downregulated in different kinds of cardiovascular diseases (Mehel et al., 2013; Smith et al., 1998; Wu et al., 2015). However, the cAMP dynamic and the role of PDEs have not been investigated in CPVT.

In this project, cAMP levels and activity of PDE2, 3 and 4 in CPVT(a, b, c, d)-CMs were investigated and compared with Ctrl-CMs. Levels of cAMP in cytosol and at the RYR2 microdomain in $\operatorname{CPVT}(\mathrm{a}, \mathrm{b}, \mathrm{c}, \mathrm{d})-\mathrm{CMs}$ and Ctrl-CMs had been examined in cooperation with Prof. Nikolaev. Several cAMP microdomains have been proposed in CMs so far; one of them is located at RYR2. In this study, the biosensors Epac1-camps and Epac1-camps-JNC were used to study the cAMP levels in cytosol and at the RYR2 microdomain, respectively. The biosensor Epac1-camps was generated by the group of Nikolaev in 2004 (Nikolaev et al., 2004), while the sensor Epac1-camps-JNC was recently generated by Konrad Götz in his doctoral thesis (Götz, 2014). While Epac1-camps is distributed throughout the cytosol, Epac1-camps-JNC is associated with RYR2 in the SR membrane.

\subsubsection{ISO concentration-dependent FRET reaction}

In the adult human heart, the $\beta 1-\mathrm{AR}$ is the predominant receptor (Brodde, 1991; Wallukat, 2002), which is located all over the cell surface, while $\beta 2-\mathrm{AR}$ is only located in the T-tubules of CMs (Nikolaev et al., 2010). It has been reported that in adult murine CMs, $\beta 2$-AR-generated cAMP is more locally confined near the RYR2, while $\beta 1$-AR-generated cAMP defuses broadly in the cell (Nikolaev et al., 2006; Nikolaev et al., 2010). Due to the relative lower maturation degree, the absence of T-tubules in iPSC-CMs is associated with the lack of regular organization of Ltype $\mathrm{Ca}^{2+}$ channel-RYR2 complexes (Kane et al., 2015). Therefore, both the $\beta 1-\mathrm{AR}$ and the $\beta 2-$ AR were stimulated by ISO simultaneously in this study.

The $\beta$-AR system is playing a predominant role in functional regulation of the heart by mediating the effects of endogenous catecholamines. To investigate whether the Epac1-camps- and Epac1camps-JNC-transfected Ctrl- and CPVT-CMs had a concentration-dependent reaction to different ISO concentration, FRET measurement was applied. The change in FRET after 
stimulation with $1 \mathrm{nM}$ (Figure 39A, B) or $10 \mathrm{nM}$ (Figure 39D, E) ISO as a percentage of the maximum change of the measurement (after IBMX treatment) was investigated by using both Epac1-camps and Epac1-camps-JNC sensors.

Representative original traces of YFP and CFP were shown for $1 \mathrm{nM}$ ISO-induced minor reaction (Figure 39A, B) and for $10 \mathrm{nM}$ ISO-induced major change in YFP and CFP (Figure 39D, E).

The statistical data demonstrated that the change in FRET (\% max) significantly increased in Epac1-camps-transfected Ctrl-CMs (25.9 \pm 1.4$)$, CPVTa-CMs (32.9 \pm 1.5$)$, CPVTb-CMs (27.9 \pm 1.6), CPVTc-CMs (26.8 \pm 1.2$)$, and CPVT-d-CMs $(23.1 \pm 1.3)$ after $10 \mathrm{nM}$ ISO treatment, when compared to $1 \mathrm{nM}$ ISO-challenged Ctrl-CMs $(9.1 \pm 0.9)$, CPVTa-CMs (12.9 \pm 0.8), CPVTbCMs $(11 \pm 1.2)$, CPVTc-CMs (14 \pm 0.9$)$, and CPVTd-CMs (12.2 \pm 1.2$)$ (Figure 39C).

The statistical data also demonstrated that the change in FRET ( $\%$ max) significantly increased

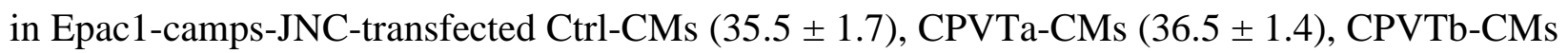
$(40.45 \pm 1.6)$, CPVTc-CMs $(40.1 \pm 1.4)$, and CPVTd-CMs $(28.33 \pm 1.6)$ after $10 \mathrm{nM}$ ISO treatment, when compared to $1 \mathrm{nM}$ ISO-challenged Ctrl-CMs (24.2 \pm 1.6$)$, CPVTa-CMs (22.9 \pm 1.5), CPVTb-CMs (19.9 \pm 1.3$)$, CPVTc-CMs (19.3 \pm 1.3$)$, and CPVTd-CMs (20.1 \pm 1.2$)$ (Figure 39F).

The FRET change in Epac1-camps-expressed CPVTa-CMs (32.97 \pm 1.5$)$ was significantly higher when compared to Ctrl-CMs $(25.91 \pm 1.4)$, indicating that the cytosolic cAMP level was higher in CPVTa-CMs than in Ctrl-CMs after $10 \mathrm{nM}$ ISO treatment. Moreover, the FRET change in Epac1-camps-JNC-expressed CPVTb- $(40.45 \pm 1.6)$ and CPVTc-CMs (40.14 \pm 1.5$)$ were significantly higher when compared to Ctrl-CMs $(35.47 \pm 1.7)$, indicating that the cAMP levels at the RYR2 microdomain were higher in these CPVT-CMs than in Ctrl-CMs after $10 \mathrm{nM}$ ISO treatment. Interestingly, the FRET change in Epac1-camps-JNC-expressed CPVTd-CMs (28.33 $\pm 1.6)$ was significantly lower when compared to Ctrl-CMs $(35.47 \pm 1.7)$, indicating that the cAMP level at the RYR2 microdomain was lower in CPVTd-CMs than in Ctrl-CMs after $10 \mathrm{nM}$ ISO treatment (Figure 39C, F). 
These data indicate that the FRET changes of Epac1-camps- and Epac1-camps-JNC-transfected Ctrl-CMs and CPVT-CMs are concentration-dependent for ISO. Therefore, this method can be used to predict the activities of different PDEs.
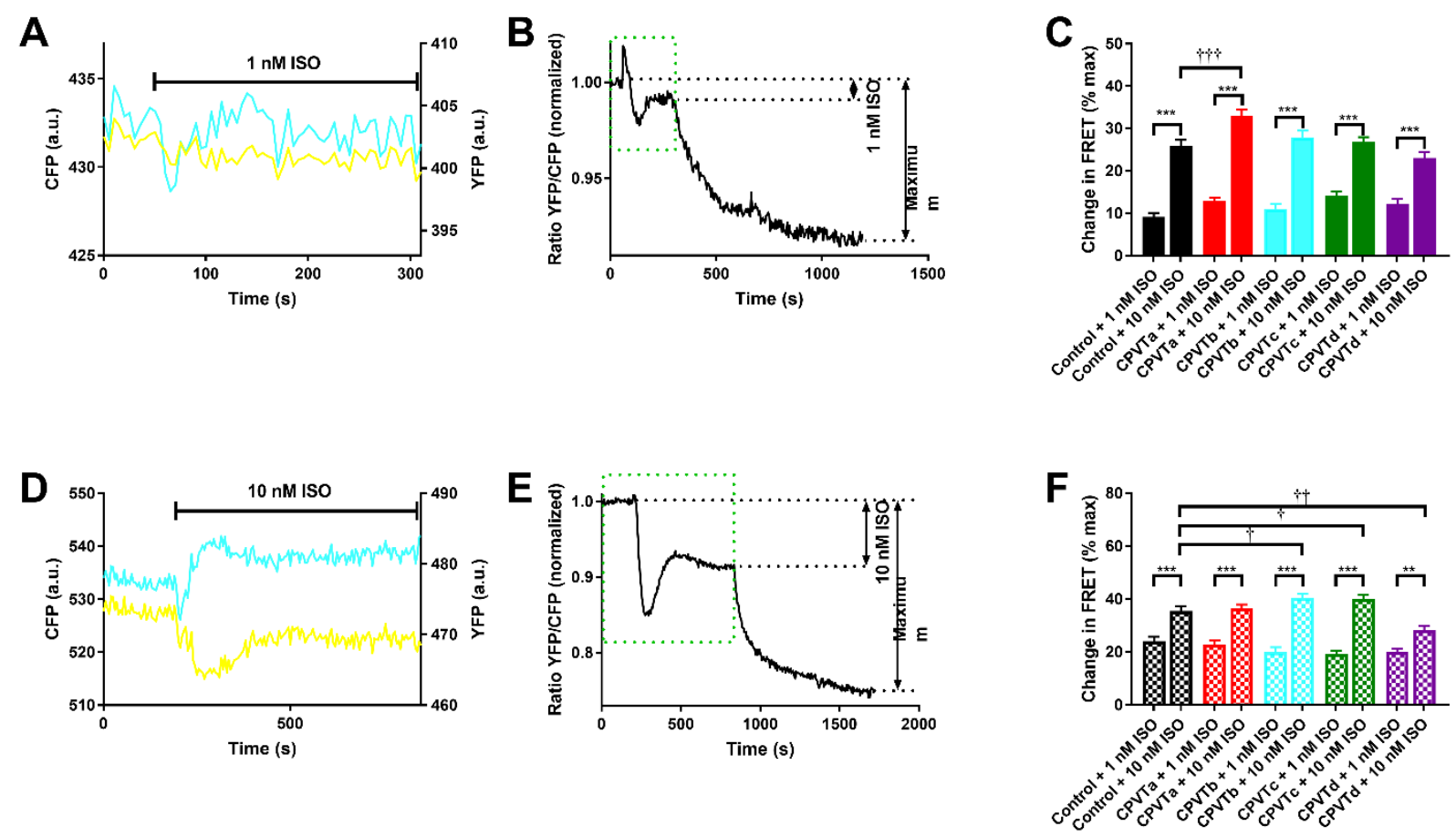

Figure 39. Different FRET changes in Epac1-camps- and Epac1-camps-JNC-transfected CMs induced by different ISO concentrations. (A, D) Representatively shown single YFP and CFP intensity recordings of the Epac1-camps-transfected CPVTd-CM (A) and Ctrl-CM (D). There was no obvious change in CFP (donor) intensity but a slight decrease in YFP (acceptor) intensity when the cell was challenging with $1 \mathrm{nM}$ ISO (A). After treated with $10 \mathrm{nM}$ ISO, cAMP caused an increase in CFP intensity and a decrease in YFP intensity (D). Change in FRET is shown as a percentage of the maximum change after the IBMX treatment to block all PDE activities during the measurement (B, E). For the Epac1camps-transfected Ctrl-, CPVTa-, CPVTb-, CPVTc- and CPVTd-CMs, the changes of FRET significantly increased in $10 \mathrm{nM}$ ISO when compared to $1 \mathrm{nM}$, respectively $(\mathbf{C})$. For the Epac1-camps-JNC-transfected Ctrl-, CPVTa-, CPVTb-, CPVTc- and CPVTd-CMs, the changes of FRET also significantly increased in $10 \mathrm{nM}$ ISO when compared to $1 \mathrm{nM}$ respectively $(\mathbf{F})$. For these $1 \mathrm{nM}$ and $10 \mathrm{nM}$ ISO-challenged Epac1camps-transfected CMs experiments, numbers of Ctrl-, CPVTa-, CPVTb-, CPVTc- and CPVTd-CMs equal to $17,44,27,86,20,39,39,66,17$ and 52, respectively (C, from left to right). For these $1 \mathrm{nM}$ and $10 \mathrm{nM}$ ISO-challenged Epac1-camps-JNC-transfected CMs experiments, numbers of Ctrl-, CPVTa-, CPVTb-, CPVTc- and CPVTd-CMs equal to 17, 38, 30, 88, 15, 36, 37, 66, 14 and 47, respectively (F, from left to right).

cAMP production is counterbalanced by hydrolytic activity of PDEs, which can shape a cAMPmediated signal after $\beta$-adrenergic stimulation. Since PDE2, PDE3, and PDE4 represent the dominant PDE families in the heart, the following experiments focused on PDE2, PDE3 and 
PDE4 inhibition to investigate cAMP degradation by different PDE hydrolytic activities in Epac1-camps- and Epac1-camps-JNC-transfected Ctrl-CMs and CPVT-CMs.

\subsection{2 cAMP degradation by PDE2 hydrolytic activity in iPSC-CMs after $\beta$-adrenergic stimulation}

To monitor the contribution of PDE2 to cAMP degradation, beating iPSC-CMs expressing Epac1-camps or Epac1-cams-JNC were challenged with $10 \mathrm{nM}$ ISO first, then with $100 \mathrm{nM}$ Bay 60-7550 to inhibit PDE2 and last with $100 \mu \mathrm{M}$ IBMX to block all PDEs.

To avoid imprecise quantification of PDE contributions, only the percentage of individual PDE inhibitor effects, without the ISO portion, from the total PDE inhibition by the unselective inhibitor IBMX was quantified. The contribution of PDE2 to cAMP degradation in cytosol after $\beta$-adrenergic stimulation was significantly higher in Ctrl-CMs $(27.56 \pm 2.277, \mathrm{n}=18)$ than in CPVTa-CMs (15.41 $\pm 1.143, \mathrm{n}=34)$, CPVTb-CMs $(9.48 \pm 1.54, \mathrm{n}=13)$, CPVTc-CMs $(15.07 \pm$ $1.419, \mathrm{n}=28)$ and CPVTd-CMs (7.887 $\pm 1.146, \mathrm{n}=16)$ (Figure 40C).

A

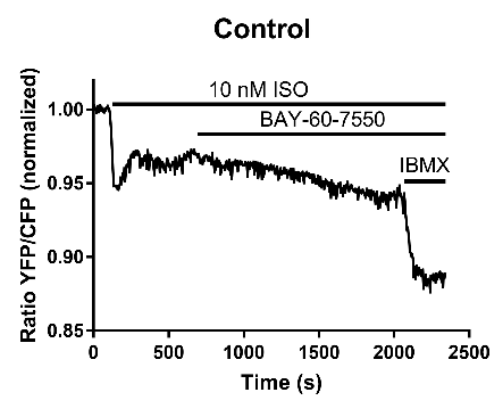

B

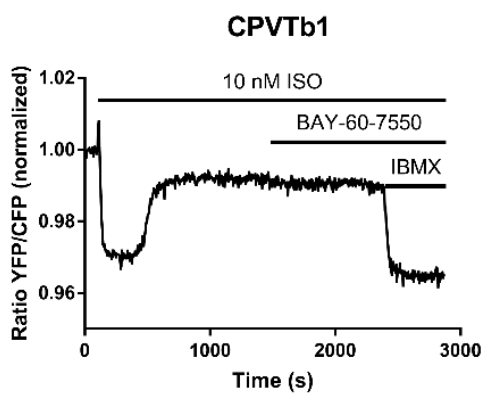

C

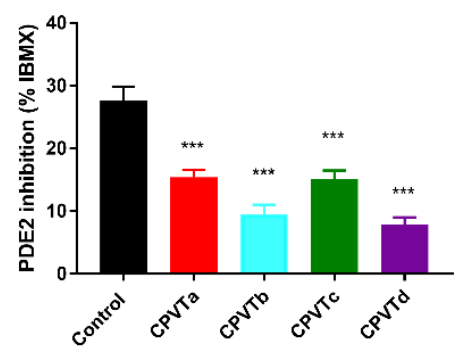

Figure 40. cAMP-PDE2 activity in cytosol after $\boldsymbol{\beta}$-adrenergic stimulation. Beating CMs expressing the Epac1-camps were stimulated with ISO (10 nM), following by Bay 60-7550 (100 nM) to block PDE2 and IBMX $(100 \mu \mathrm{M})$ to block all PDEs fully. Representative FRET ratio traces were shown for Ctrl-CMs (A), and for CPVTb1-CMs (B). (C) PDE2 contribution to cAMP hydrolysis in the bulk cytosol was significantly lower in $\operatorname{CPVT}(\mathrm{a}, \mathrm{b}, \mathrm{c}, \mathrm{d})$-CMs when compared to Ctrl-CMs. Data were shown as means \pm SEM. n=18, 34, 13, 28 and 16 for Ctrl-, CPVTa-, CPVTb-, CPVTc- and CPVTd-CMs, respectively. 
In addition, the contribution of PDE2 to cAMP degradation in the RYR2 compartment after $\beta$ adrenergic stimulation was significantly higher in Ctrl-CMs $(39.98 \pm 2.461, \mathrm{n}=15)$ than in CPVTa-CMs (14.19 $\pm 1.644, \mathrm{n}=26)$, CPVTb-CMs (25.21 $\pm 2.78, \mathrm{n}=13)$, CPVTc-CMs (17.32 \pm 1.397, $\mathrm{n}=25)$ and CPVTd-CMs (8.606 $\pm 1.232, \mathrm{n}=11)$ (Figure 41C).

A

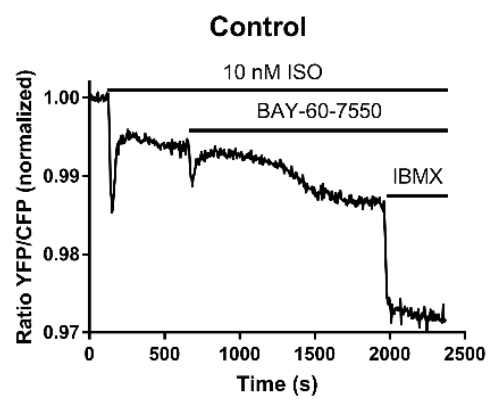

B

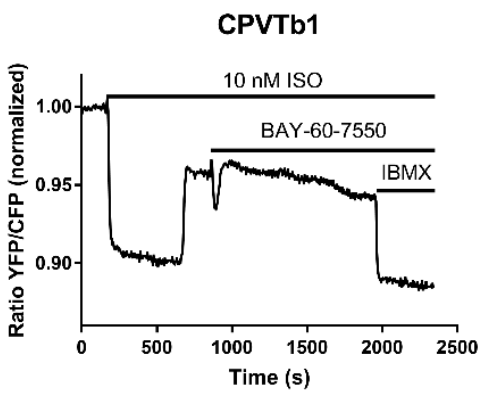

C

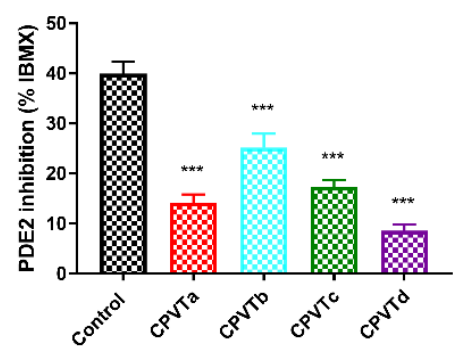

Figure 41. cAMP-PDE2 activity in the RYR2 compartment after $\beta$-adrenergic stimulation. Beating CMs expressing the Epac1-camps-JNC were stimulated with ISO $(10 \mathrm{nM})$, following by Bay 60-7550 $(100 \mathrm{nM})$ to block PDE2 and IBMX $(100 \mu \mathrm{M})$ to block all PDEs fully. Representative FRET ratio traces were shown for Ctrl-CMs (A), and for CPVTb1-CMs (B). Compared to Ctrl-CMs, CPVT(a, b, c, d)-CMs revealed significant lower PDE2 contribution to cAMP hydrolysis in the RYR2 compartment (C). Data were shown as means \pm SEM. $n=15,26,13,25,11$ for Ctrl-, CPVTa-, CPVTb-, CPVTc- and CPVTdCMs, respectively.

\subsection{3 cAMP degradation by PDE3 hydrolytic activity in iPSC-CMs after $\beta$-adrenergic stimulation}

To monitor the contribution of PDE3 to cAMP degradation, beating iPSC-CMs expressing Epac1-camps or Epac1-camps-JNC were challenged with $10 \mathrm{nM}$ ISO first, then with $10 \mu \mathrm{M}$ cilostamide to inhibit PDE3 and last with $100 \mu \mathrm{M}$ IBMX to block all PDEs.

Similar to PDE2, the contribution of PDE3 to cAMP degradation in cytosol after $\beta$-adrenergic stimulation was significantly higher in Ctrl-CMs $(35.34 \pm 1.911, \mathrm{n}=19)$ than in CPVTa-CMs (9.17 $\pm 1.539, \mathrm{n}=23)$, CPVTb-CMs $(15.61 \pm 2.639, \mathrm{n}=13)$, CPVTc-CMs $(14.7 \pm 1.373, \mathrm{n}=27)$ and CPVTd-CMs (11.49 $\pm 1.634, \mathrm{n}=18)$ (Figure 42C). 
A

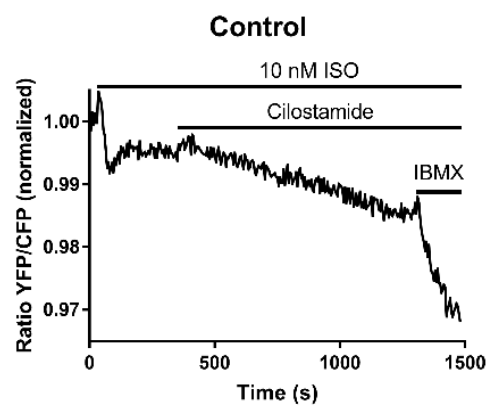

B

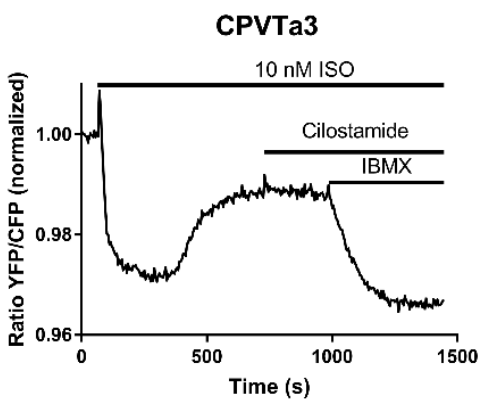

C

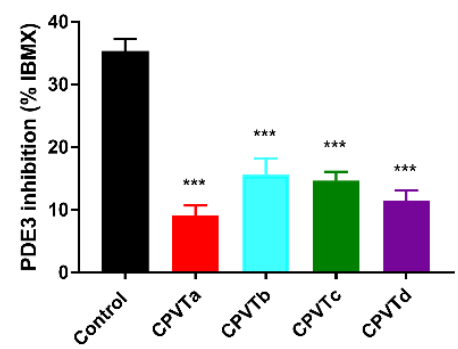

Figure 42. cAMP-PDE3 activity in cytosol after $\boldsymbol{\beta}$-adrenergic stimulation. Beating CMs expressing the Epac1-camps were stimulated with ISO $(10 \mathrm{nM})$, following by cilostamide $(10 \mu \mathrm{M})$ to block PDE3 and IBMX $(100 \mu \mathrm{M})$ to block all PDEs fully. Representative FRET ratio traces were shown for Ctrl-CMs (A), and for CPVTa3-CMs (B). (C) Quantification of FRET responses was shown. CPVT(a, b, c, d)-CMs demonstrated a significant lower PDE3 contribution to cAMP hydrolysis in the bulk cytosol compared to Ctrl-CMs. Data were shown as means \pm SEM. n=19, 23, 13, 27 and 18 for Ctrl-, CPVTa-, CPVTb-, CPVTc- and CPVTd-CMs, respectively.

The contribution of PDE3 to cAMP degradation in the RYR2 compartment after $\beta$-adrenergic stimulation was also significantly higher in Ctrl-CMs $(49.84 \pm 2.469, \mathrm{n}=17)$ than in CPVTa-CMs (16.31 $\pm 1.988, \mathrm{n}=30)$, CPVTb-CMs $(21.13 \pm 3.241, \mathrm{n}=11)$, CPVTc-CMs $(20.31 \pm 2.592, \mathrm{n}=25)$ and CPVTd-CMs $(8.163 \pm 1.329, \mathrm{n}=16)$ (Figure 43C).

A

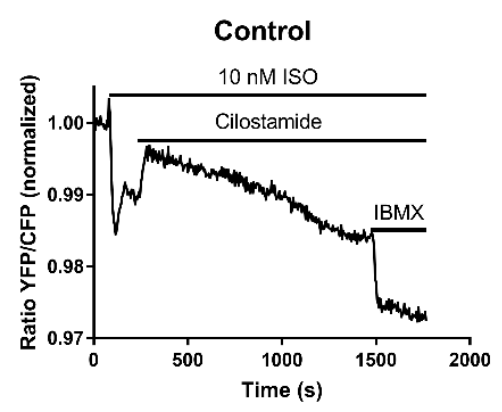

B

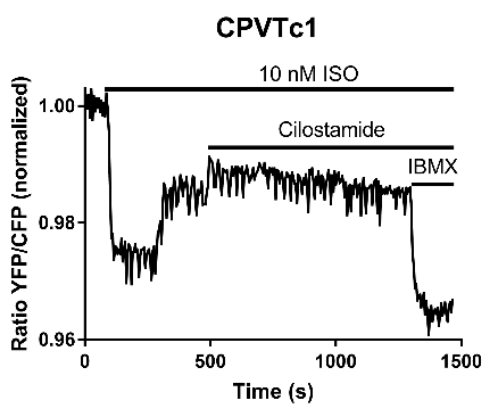

C

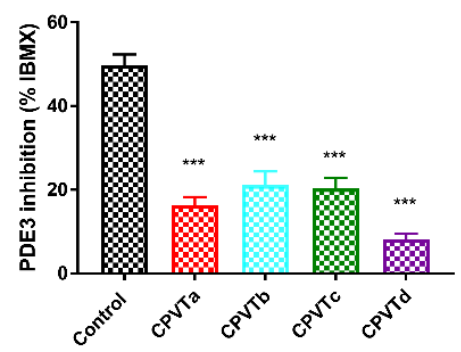

Figure 43. cAMP-PDE3 activity in the RYR2 compartment after $\beta$-adrenergic stimulation Beating CMs expressing the Epac1-camps-JNC were stimulated with ISO $(10 \mathrm{nM})$, following cilostamide (10 $\mu \mathrm{M})$ to block PDE3 and IBMX $(100 \mu \mathrm{M})$ to block all PDEs fully. Representative FRET ratio traces were shown for Ctrl- (A), and for CPVTc1-CMs (B). Compared to Ctrl-CMs, CPVT(a, b, c, d)-CMs showed significant lower PDE3 contribution to cAMP hydrolysis in the RYR2 compartment (C). Data were 
shown as means \pm SEM. $\mathrm{n}=17,30,11,25$ and 16 for Ctrl-, CPVTa-, CPVTb-, CPVTc- and CPVTd-CMs, respectively.

\subsection{4 cAMP degradation by PDE4 hydrolytic activity in iPSC-CMs after $\beta$-adrenergic stimulation}

Pilot experiments showed that stimulation with $10 \mathrm{nM}$ ISO led to an increase in cAMP, and addition of the PDE4 inhibitor rolipram further raised cAMP concentration to levels that were high enough to saturate the FRET sensors (data not shown). To measure the dynamic range of the FRET sensors, PDE4 inhibition was performed upon stimulation with $1 \mathrm{nM}$ ISO, which can induce smaller changes in cAMP concentration than $10 \mathrm{nM}$ in all iPSC-CMs no matter in cytosol or at the RYR2 compartment (Figure 39), allowing visualization of the complete FRET response to rolipram.

Different from PDE2 and PDE3 activities in CPVT-CMs, the contribution of PDE4 to cAMP degradation in cytosol after $1 \mathrm{nM}$ ISO stimulation was significantly lower in CPVTb-CMs (36.5 $\pm 4.77, \mathrm{n}=20)$ and CPVTc-CMs $(45.67 \pm 4.108, \mathrm{n}=39)$, when compared to Ctrl-CMs $(71.72 \pm$ 3.749, $\mathrm{n}=17)$. This was not the case for CPVTa-CMs $(74.23 \pm 2.378, \mathrm{n}=27)$ and CPVTd-CMs $(66.05 \pm 3.94, \mathrm{n}=14)$ (Figure 44D). 

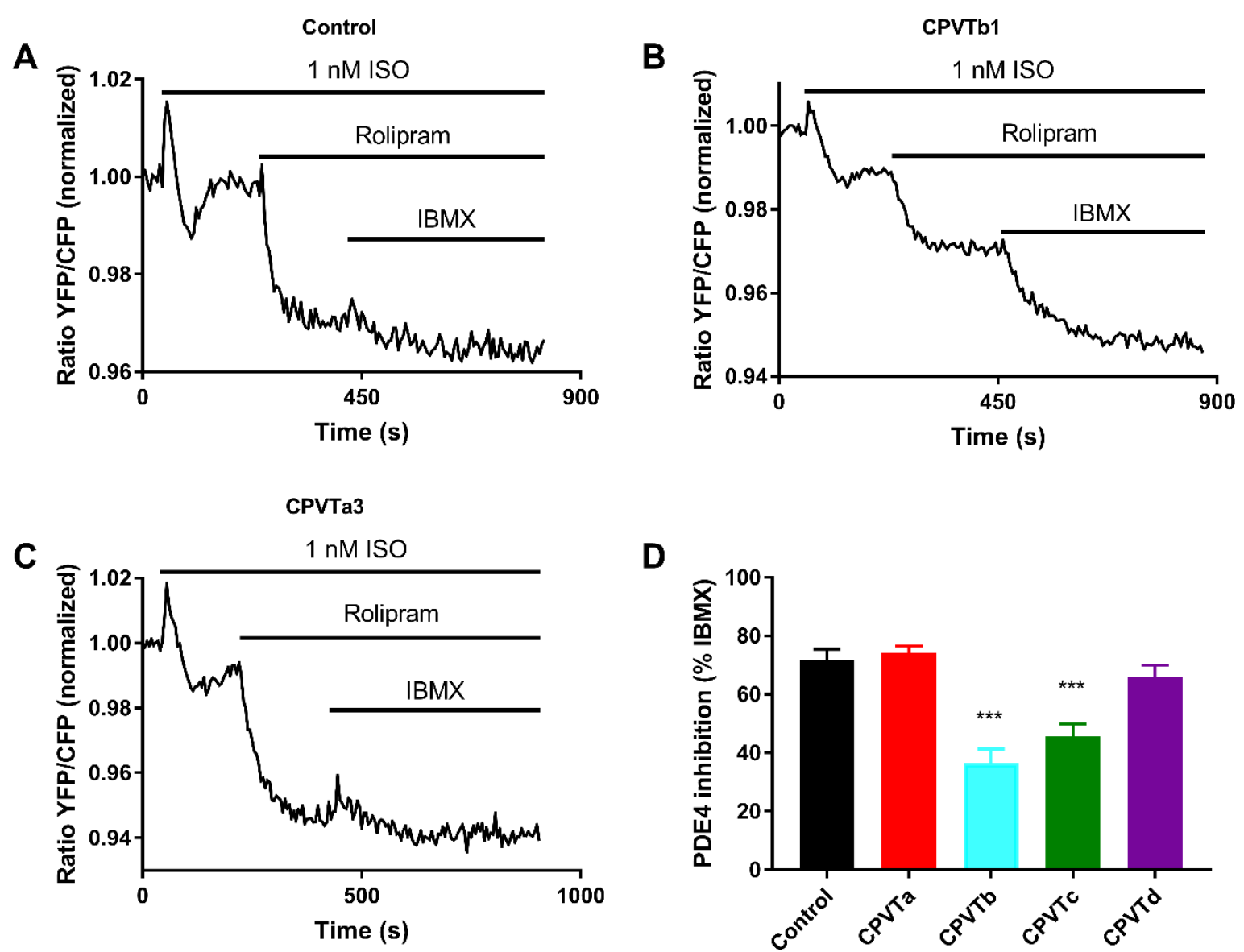

Figure 44. cAMP-PDE4 activity in cytosol after $\boldsymbol{\beta}$-adrenergic stimulation. Beating CMs expressing the Epac1-camps were stimulated with ISO $(1 \mathrm{nM})$, following by rolipram $(10 \mu \mathrm{M})$ to block PDE4 and IBMX $(100 \mu \mathrm{M})$ to block all PDEs fully. Representative FRET ratio traces were shown for Ctrl-CMs (A), CPVTb1-CMs (B) and CPVTa3-CMs (C). (D) Quantification of FRET responses was shown. CPVTband CPVTc-CMs revealed a significant lower PDE4 contribution to cAMP hydrolysis in the bulk cytosol compared to Ctrl-CMs. Data were shown as means \pm SEM. n=17, 27, 20, 39 and 14 for Ctrl-, CPVTa-, CPVTb-, CPVTc- and CPVTd-CMs, respectively.

Interestingly, the PDE4 contribution to cAMP degradation at the RYR2 compartment in CPVTcCMs (55.92 $\pm 4.379, \mathrm{n}=37)$ and CPVTd-CMs $(34.26 \pm 4.865, \mathrm{n}=12)$ was significantly lower, but not in CPVTa-CMs (82.05 $\pm 1.97, \mathrm{n}=30)$ and CPVTb-CMs (73.16 \pm 5.574, $\mathrm{n}=15)$, when compared to Ctrl-CMs (73.53 $\pm 5.103, \mathrm{n}=17)$ after $1 \mathrm{nM}$ ISO stimulation (Figure 45D). 

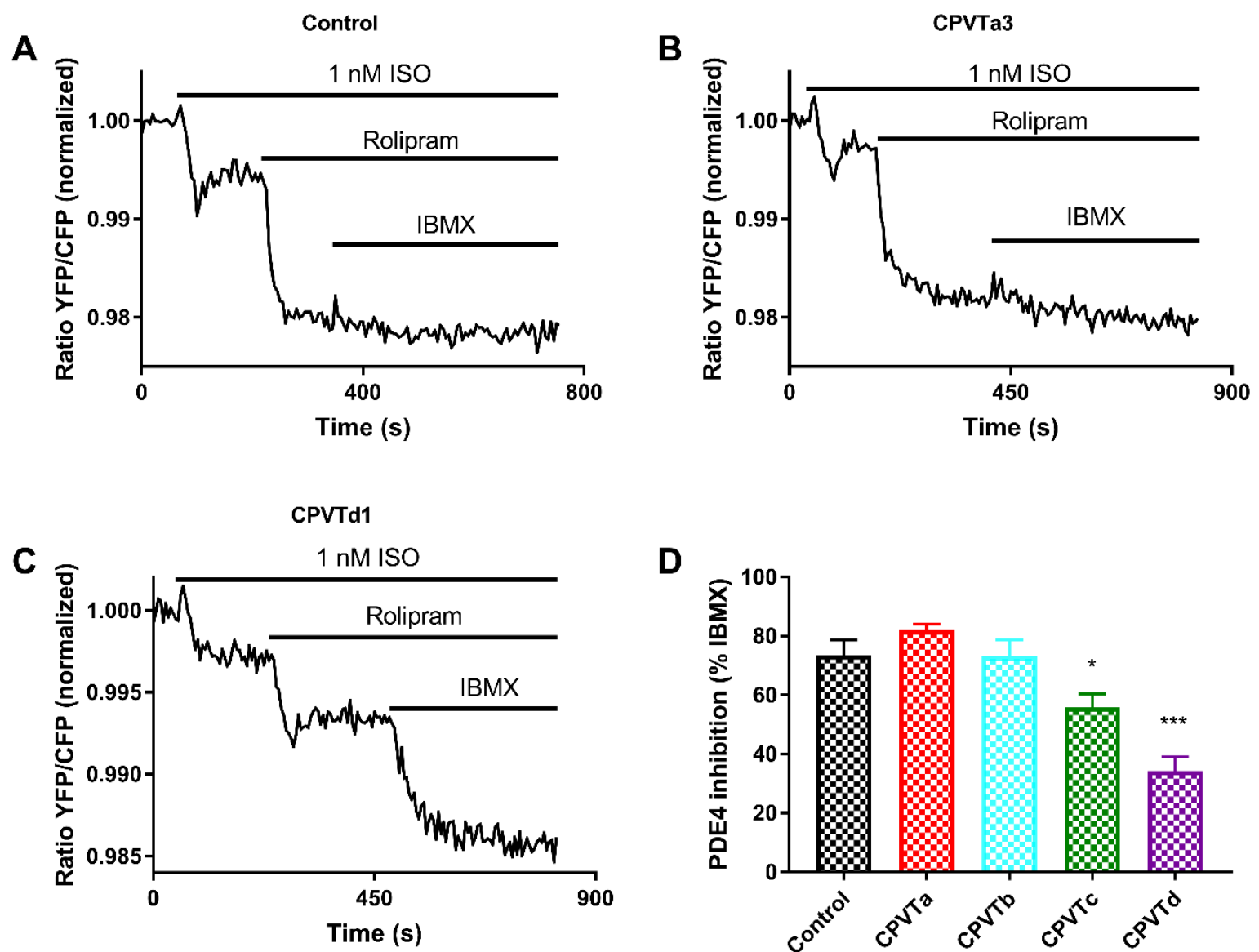

Figure 45. cAMP-PDE4 activity in the RYR2 compartment after $\beta$-adrenergic stimulation. Beating CMs expressing the Epac1-camps-JNC were stimulated with ISO $(1 \mathrm{nM})$, following by rolipram $(10 \mu \mathrm{M})$ to block PDE4 and IBMX $(100 \mu \mathrm{M})$ to block all PDEs fully. Representative FRET ratio traces were shown for Ctrl-CMs (A), CPVTa3-CMs (B) and CPVTd1-CMs (C). (D) Quantification of FRET responses was shown. CPVT(c, d)-CMs exhibited a significant lower PDE4 contribution to cAMP hydrolysis in the RYR2 compartment compared to Ctrl-CMs. Data were shown as means \pm SEM. $n=17$, 30, 15, 37 and 12 for Ctrl-, CPVTa-, CPVTb-, CPVTc- and CPVTd-CMs, respectively. 


\section{Discussion}

CPVT is characterized by abnormal calcium handling, ventricular arrhythmias, and sudden cardiac death induced by exercise or acute emotion in individuals without structural cardiac abnormalities. The overwhelming majority (more than 76\%) of the identified RYR2 mutations (more than 150) were classified in clusters in four distinct regions. However, the underlying electrophysiological mechanisms have not been completely understood. Patient-specific hiPSCs as a renewable and reliable source for CMs provide the possibility to study the pathophysiology of genetic diseases in vitro.

The aim of this thesis was to investigate whether CMs differentiated from hiPSCs generated from CPVT patients could recapitulate electrophysiological features of the disease in vitro as well as to test the effect of antiarrhythmic drugs on the function of CPVT-iPSC-CMs. In addition, the cAMP dynamic was investigated.

Somatic cells from skin biopsies of CPVT patients carrying the RYR2 mutation in domain a (R420W), domain b (A2254V), domain c (E4076K) or domain d (H4742Y) were isolated and reprogrammed into patient-specific iPSCs. The generated Ctrl- and CPVT-iPSCs exhibited hESC-like characteristics, demonstrated by pluripotency-related gene expression, in vitro differentiation capacity, and teratoma formation in vivo. Furthermore, both Ctrl- and CPVThiPSCs were able to differentiate into functional CMs, which were shown by spontaneous contraction and cardiac-specific protein expression.

Electrophysiological analyses showed that the CPVT-CMs recapitulated the phenotype of CPVT both by patch-clamp assessment and by MEA assessment. The single CPVTd-CMs showed a unique EAD phenotype in basal condition and ISO-challenged condition whereas CPVTa-, CPVTb- and CPVTc-iPSC-CMs exhibited DADs and DAD-induced TAs, which were significantly enhanced after the ISO treatment. In the monolayer cultures, all CPVT-CMs revealed a significantly increased number of PVC- and VT-like events after the ISO treatment. In contrast, no increased appearance of DADs, EADs, DAD- or EAD-induced TAs, and PVC- and VT-like events was observed in Ctrl-iPSC-CMs after the ISO treatment. The stress-induced arrhythmia can be specifically alleviated by the treatment with four different therapeutic drugs (flecainide, dantrolene, rycal1 and rycal2). 
The activities of PDE2 and PDE3 in all CPVT(a, b, c, d)-CMs were lower than in Ctrl-CMs no matter in cytosol or at the RYR2 compartment through FRET measurement. However, for PDE4, there was a lower activity in cytosol in CPVTb- and CPVTc-iPSC-CMs when compared to CtrliPSC-CMs, and a lower activity at the RYR2 compartment in CPVTc- and CPVTd-iPSC-CMs when compared to Ctrl-iPSC-CMs.

\subsection{Generation and characterization of hiPSCs and iPSC-CMs differentiation}

It has been ten years since Shinya Yamanaka published his milestone work for cell reprogramming (Takahashi and Yamanaka, 2006). To address the safety issues arisen from harboring integrated exogenous sequences in the target cell genome, a number of methods have been developed to produce iPSCs with potentially reduced risks, which included nonintegrational adenoviral (Stadtfeld et al., 2008; Zhou and Freed, 2009), non-integrational Sendai viral (Fujie et al., 2014; Fusaki et al., 2009), synthetic mRNA (Warren et al., 2010; Yakubov et al., 2010) and cell-penetrating protein reprogramming (Kim et al., 2009; Zhou et al., 2009). The Ding's group showed that somatic cells can be reprogrammed by combining OCT4 and KLF4 with small-molecule compounds (Shi et al., 2008) to improve induction of human iPSCs through chemical compound treatment (Lin et al., 2009), and by using recombinant proteins (Zhou et al., 2009). Finally, a group led by Hongkui Deng reported that they were able to get $0.2 \%$ of cells to convert to iPSCs (results comparable to those from standard virus iPSC production techniques) by using a cocktail of seven compounds (Hou et al., 2013).

In this study, the Sendai virus system containing the four Yamanaka factors OCT4, SOX2, KLF4, and $C-M Y C$ was used for the reprogramming of fibroblasts from healthy donors and CPVT patents into transgene-free hiPSCs. The Sendai virus system enables RNA-based gene delivery of pluripotency-associated factors and shows a high efficiency (Fujie et al., 2014; Fusaki et al., 2009).

The hiPSCs generated in the present study showed typical hiPSC-like characteristics with regards to their morphology, expression of pluripotency-related marker genes, including OCT4, SOX2, NANOG, LIN28, GDF3 and FOXD3 (Figure 12), as well as expression of the 
pluripotency-related factors at protein level, including NANOG, OCT4, SOX2, LIN28, SSEA4 and TRA1-60 (Figure 13).

Furthermore, CPVTa2, CPVTa3 and CPVTd1 iPSCs expressed markers specific for all three germ layers upon in vitro differentiation, demonstrating that they are truly pluripotent. The ability to differentiate into almost all tissue types is the hallmark of human pluripotent stem cells (Itskovitz-Eldor et al., 2000). Gene expression analyses demonstrated the expression of germ layer-specific markers $A F P, A L B, \alpha-M H C, c T N T, S Y P$ and $T H$ on RNA level, indicating the ability of CPVT(a2, a3, d1)-iPSCs to differentiate into the all three germ layers (endodermal, mesodermal, and ectodermal lineages) (Figure 14). Protein expression analyses indicated the expression of germ layer-specific markers AFP, SMA and $\beta$ III-tubulin on protein level, making sure the ability of $\operatorname{CPVT}(\mathrm{a} 2, \mathrm{a} 3, \mathrm{~d} 1)$-iPSCs to differentiate into derivatives of all three germ layers (endodermal, mesodermal, and ectodermal lineages) (Figure 15). Apart from in vitro differentiation experiments, teratoma formation assays are the "gold standard" for demonstrating the differentiation potential of human pluripotent stem cells (Buta et al., 2013; Hentze et al., 2009). In vivo differentiation of CPVT(a2, a3, d1)-iPSCs (Figure 16) revealed tissue-specific cells, including derivatives of all three germ layers, such as intestinal tissue (endoderm), cartilage and muscle cells (mesoderm) and neural rosettes (ectoderm).

Furthermore, sequencing analyses confirm that CPVTa2 and a3-hiPSCs carry a missense single nucleotide polymorphism on position 1258 , with a cytosine on the one allele and a thymine on the other. The variant leads to a codon for tryptophan (R420W). Sequencing analyses also conform that CPVTd1-hiPSCs contain a missense single nucleotide polymorphism on position 14224 , with a cytosine on the one allele and a thymine on the other. This variant leads to a codon for tyrosine (H4742Y) (Figure 18).

Taken together, the generated patient-specific hiPSCs have similar characteristics of human pluripotent stem cells as previously reported and fulfill the criteria defining fully reprogrammed hiPSCs with a stable karyotype. Because of their origin (somatic cells), their further use for research purposes does not raise ethical concerns as human embryonic stem cells do.

To establish a disease modeling system from CPVT patients, generation of patient-specific functional CMs is the first step. The general idea of direct reprogramming of fibroblasts into 
functional CMs by defined factors was materialized by Ieda et al. (Ieda et al., 2010). Zhang et al. recently reported that the expandable cardiovascular progenitor cells reprogrammed from fibroblasts were a powerful system to use as heart disease model and to provide strategies for regenerative medicine in the heart (Zhang et al., 2016). The strategy of direct convention of fibroblasts into functional CMs by small molecules bypass the pluripotency stage (Cao et al., 2016) provides a new cost- and time-effective way to perform cardiovascular disease modeling and therapeutic implications, even though there is no corresponding literature published nowadays.

In the present study, directed cardiac differentiation methods are used for the generation of functional CMs. To produce sufficient CMs for in vitro analyses, upscaling generation of CMs from hiPSCs is essential. In this study, the directed cardiac differentiation method (Lian et al., 2012; Lian et al., 2013) is combined with a mechanical selection (removal of non-cardiac clusters) for the generation of CMs in large quantities and with a high purity (> 80\%). Although the lactate replacement method is widely used for CM selection (Tohyama et al., 2013), side effects such as an altered expression profile and maturation due to metabolic selection are still not clearly investigated. Immunofluorescence staining demonstrated that hiPSC-derived CMs expressed sarcomeric and gap junction proteins $\mathrm{Cx} 43$, which are critical for cardiac function. This study shows a cardiac-specific protein expression in CPVT(a2, a3, d1)-CMs similar to CtrlCMs, represented by cTNT, MLC2a, MLC2v, $\alpha$-actinin, and Cx43 staining. This is consistent with the clinical findings that patients diagnosed with CPVT have a structurally normal heart (Lieve et al., 2016).

Electrophysiological recordings in both Ctrl- and CPVT-CMs reveal, on the basis of distinct classes of APs, the three major cardiac subtypes: embryonic ventricular-, atrial-, and nodal-like CMs, similar to those found in hiPSC-derived CMs (Jung et al., 2012). This classification is based on the shape and properties of the APs, such as the RMP, APA, $\mathrm{V}_{\max }$, and prominence of a plateau phase. Using the directed cardiac differentiation protocol, the majority of the cardiac subtype is ventricular-like with typical AP characteristics (Figure 22), consistent with the immunostaining results showing that majority of CMs are MLC2v positive (Figure 20). During the development of the human heart, MLC2v is exclusively expressed in ventricular CMs (Chuva de Sousa Lopes et al., 2006). 
Therefore, ventricular CMs derived from patient-specific hiPSCs carrying the mutations by using the directed cardiac differentiation method are suitable for CPVT disease modeling.

\subsection{CPVT-CMs can recapitulate electrophysiological phenotypes and imply distinguish pathologies}

Since $R Y R 2$ is a very large gene, it is not possible until now to exogenously introduce the $R Y R 2$ mutations into adult CMs to assess the resulting consequences of genetic modifications in RYR2 in vitro. Most studies of the pathophysiological mechanisms are conducted in cellular heterologous overexpression studies or in knock-in mouse models (Medeiros-Domingo et al., 2009; Priori and Chen, 2011). Until now, only a few mutations (about 15\%) have been investigated in heterologous overexpression studies or knock-in mouse models (Kashimura et al., 2010; Sedej et al., 2010; Zhang et al., 2013b). The different results from the previous studies make it difficult to elucidate the underlying mechanisms of calcium leak from different RYR2 mutations. Although heterologous expression systems and animal models (Lehnart et al., 2008) have shown important insights into the CPVT pathogenesis, the lack of in vitro sources for human CMs and the inability to model patient-specific variations of the disease significantly impedes the investigation of this disease. Therefore, further investigations using patient-specific cells are necessary. The new technology of iPSCs allows us to study human cardiovascular diseases using patient-specific cells in vitro (Itzhaki et al., 2012).

Since ECG is broadly used to quantify arrhythmic events in murine CPVT mouse models (Cerrone et al., 2005; Cerrone et al., 2007; Kannankeril et al., 2006; Kobayashi et al., 2010; Lehnart et al., 2008; Song et al., 2007), the corresponding method to assess arrhythmia in iPSCCM models for CPVT is still lacking. The MEA technology seems to be a standard procedure widely used for modeling of long QT syndrome using iPSC-CMs (Egashira et al., 2012; Friedrichs et al., 2015; Itzhaki et al., 2011; Lahti et al., 2012; Matsa et al., 2011), due to noninvasive measurements of local field potential duration (or QT interval) representing the AP duration. However, for CPVT modeling, the MEA system was only used to analyze an activation map, beating frequency and variation in beat period and corrected field potential duration by using iPSC-CM aggregates but not monolayer (Itzhaki et al., 2012; Preininger et al., 2016). Some objective facts of the maturation degree of iPSC-CMs and experimental procedures made 
it hard to analyze arrhythmic events included PVC-like events and PVC-like-induced VT-like events (Figure 26).

This is the first study to present PVC-like and VT-like events in CMs derived from CPVT-iPSCs by using the MEA technology. The non-sustained ventricular arrhythmia are very common in ECG recording (Curtis et al., 2013), but rare in this study. Sinoatrial node has the potential to correct the abnormal electrical activities of ventricle in the heart. However, there is no stronger enough sinus rhythm generated in the monolayer of MEA recording to correct PVC-like events. Therefore, the PVC-like events will evolve into VT-like events but not into non-sustained ventricular tachycardia.

Traditionally, the mechanisms for CPVT arrhythmogenesis required RYR2 affected by the gainof-function mutations (Blayney and Lai, 2009; Kontula et al., 2005; Liu et al., 2009; Marks, 2013; Priori and Chen, 2011; Priori and Napolitano, 2005; Sumitomo, 2015; Wehrens and Marks, 2003). Premature spontaneous SR $\mathrm{Ca}^{2+}$ release or $\mathrm{Ca}^{2+}$ spillover occurs under conditions of SR $\mathrm{Ca}^{2+}$ overload caused by stress. The resulting spontaneous $\mathrm{Ca}^{2+}$ elevation during diastole activates the sodium-calcium exchanger, which in turn depolarizes the cell membrane and generates DADs. DADs of sufficient amplitude then trigger APs, which give rise to the typical polymorphic VT in CPVT. However, this traditional mechanism is challenged by several other mechanisms (Liu et al., 2009; Zhao et al., 2015).

In this study, iPSC-CMs from six CPVT patients with four different mutations at four different domains (a-d) of RYR2 were investigated. The CPVTa2 and CPVTa3 iPSC lines are generated from patients harboring heterozygous mutation (R420W), which is located in the N-terminal domain (residues 1-642) (Figure 4). Cases with the R420W mutation have been reported to show arrhythmogenic right ventricular cardiomyopathy-like phenotypes of mild fatty infiltration in the heart apex (Nishio et al., 2006) despite the notion that patients with RYR2 mutations show structurally normal hearts. Different results using a knock-in mouse model of the Ryr2 mutation R420W were reported. One study showed that hypertrophy of lymphoid organs was a possible phenotypic characteristic of Ryr2 R420W mutation whereas the heart weight was not changed (Nishio et al., 2014). The other study emphasized that histology of the heart did not detect any differences between the homozygous and wild-type mice (Okudaira et al., 2014). However, the authors detected that the decay time of depolarization-induced $\mathrm{Ca}^{2+}$ transition was prolonged and 
$\mathrm{Ca}^{2+}$ transient amplitude was decreased in $\mathrm{Ryr} 2^{\mathrm{R} 420 \mathrm{~W} / \mathrm{R} 420 \mathrm{~W}}$ knock-in mouse model. Nevertheless, the point mutation R420Q causing CPVT phenotype behaves as a gain-of-function both in permeabilized and intact cells (Domingo et al., 2015). The complex mechanism of mutation R420Q goes deeper into structure level these years (Borko et al., 2014; Kimlicka et al., 2013). The RYR2 R420Q mutation has been observed ablating chloride binding, and disturbing its domain folding in the crystal structure of the N-terminal region (Borko et al., 2014; Kimlicka et al., 2013). It appears clear that the R420Q mutation destabilizes the inter-subunit interface, probably facilitating the channel opening. Recently, a CPVT-iPSC-CMs model was used to study the RYR2 ${ }^{\mathrm{R} 420 \mathrm{Q}}$ mutation compared with $\mathrm{CASQ} 2^{\mathrm{D} 307 \mathrm{H}}$ mutation, revealing several calcium handling abnormalities (Novak et al., 2015). In this study, patient-specific iPSC-CMs with the RYR2 mutation R420W, which has not been studied before in iPSC models, showed the lower beating frequency when compared to Ctrl-CMs after the ISO treatment as well as the increased number of DADs, DAD-induced TAs and PVC-like or VT-like events. These findings are in line with the data showing bradycardia, irregularity in RR intervals and increased VT-like events in Ryr $2^{\mathrm{R} 420 \mathrm{~W} / \mathrm{R} 420 \mathrm{~W}}$ knock-in mouse model after $\beta$-adrenergic stimulation (Okudaira et al., 2014). Furthermore, in our lab, we could show that ISO stimulation of fluo 4/AM-loaded CPVTa-CMs (R420W) leads to increased spontaneous $\mathrm{Ca}^{2+}$ release determined by $\mathrm{Ca}^{2+}$ spark analysis using confocal line scanning technique (L. Cyganek, unpublished data). However, when compared to Ctrl-CMs, the frequency of $\mathrm{Ca}^{2+}$ sparks in CPVTa-CMs after the ISO treatment is lower. Future study should focus on the abnormality of $\mathrm{Ca}^{2+}$ transients to better understand the underlying mechanism for RYR2 $2^{\mathrm{R} 420 \mathrm{~W}}$-caused CPVT.

Although some literatures reported that the RYR2 $2^{\mathrm{A} 2254 \mathrm{~V}}$ mutation is correlated with the clinical outcome of CPVT patients (Medeiros-Domingo et al., 2009; Postma et al., 2005; van der Werf et al., 2012a), investigation of its underlying mechanism has not been reported. Based on the latest structure analysis of porcine Ryr2 using the cryo-electron microscopy (Peng et al., 2016), A2254 residue is located in the helical domain 1 (residues 2111-2679) (Figure 4). The structure analysis of the rabbit Ryr1 in complex with its modulator FKBP12 by using single-particle electron cryomicroscopy demonstrates that FKBP12 is bound in a cleft formed by the handle, N-terminal and SPRY1/3 domains (Yan et al., 2015). Crystal structures of Ryr2 reveal that the hydrophobic cluster within SPRY1 is crucial for FKBP binding (Yuchi et al., 2015). In this study, patientspecific iPSC-CMs with the mutation RYR2 ${ }^{\mathrm{A} 2254 \mathrm{~V}}$, which has not been studied before either in 
iPSC models or in mouse knock-in models, showed the increased number of DADs, DADinduced TAs and PVC-like or VT-like events when compared to Ctrl-CMs after the ISO treatment. The beating frequency in CPVTb-CMs is comparable with that in Ctrl-CMs. Furthermore, in our lab, we could show that ISO stimulation of fluo 4/AM-loaded CPVTb-CMs (A2254V) leads to increased spontaneous $\mathrm{Ca}^{2+}$ release determined by $\mathrm{Ca}^{2+}$ spark analysis using confocal line scanning technique (L. Cyganek, unpublished data). When compared to Ctrl-CMs, the frequency of $\mathrm{Ca}^{2+}$ sparks in CPVTb-CMs after the ISO treatment is significantly higher. These findings suggest that RYR2 $2^{\mathrm{A} 2254 \mathrm{~V}}$ is a gain-of-function mutation. Future study should figure out whether the FKBP12 binding is changed in CPVTb-CMs.

Similar to RYR2 ${ }^{\mathrm{A} 2254 \mathrm{~V}}$, the underlying mechanism of CPVT caused by the RYR2 ${ }^{\mathrm{E} 4076 \mathrm{~K}}$ mutation has not been studied, although there are some literatures reporting its clinical relevance (Medeiros-Domingo et al., 2009; Postma et al., 2005; van der Werf et al., 2012a). E4076K mutation is located in the central domain of RYR2 (residues 3613-4207) (Figure 4). In addition, Xiong et al. have identified two helix-loop-helix structural (EF-hand) $\mathrm{Ca}^{2+}$ binding motifs in Ryr2 (residues 4036-4082) (Xiong et al., 1998). The residue E4076 is participating in the formation of the second EF-hand motif. Deleting the entire EF-hand motif in the central domain does not affect cytosolic $\mathrm{Ca}^{2+}$ activation of single RYR2 channels, suggesting that the EF-hand is unlikely to be the cytosolic $\mathrm{Ca}^{2+}$ sensor (Guo et al., 2016). Atomic-resolution structures of Ryr2 may unveil the $\mathrm{Ca}^{2+}$ binding sites in the central domain for cytosolic $\mathrm{Ca}^{2+}$ activation (Peng et al., 2016). Nevertheless, the EF-hand $\mathrm{Ca}^{2+}$ binding domain is important for luminal $\mathrm{Ca}^{2+}$ activation of Ryr2 and for the activation and termination of store overload-triggered $\mathrm{Ca}^{2+}$ release (Guo et al., 2016).

In this study, patient-specific iPSC-CMs with the RYR2 mutation E4076K, which has not been studied before either in iPSC models or in mouse knock-in models, showed the increased number of DADs, DAD-induced TAs and PVC-like or VT-like events when compared to Ctrl-CMs after the ISO treatment. The beating frequency in CPVTc-CMs is comparable with that in Ctrl-CMs. Furthermore, ISO stimulation of CPVTc-CMs (E4076K) leads to increased spontaneous $\mathrm{Ca}^{2+}$ release (L. Cyganek, unpublished data). When compared to Ctrl-CMs, the frequency of $\mathrm{Ca}^{2+}$ sparks in CPVTc-CMs after the ISO treatment is significantly higher. These findings suggest that E4076K, similar to A2254V, is a gain-of-function mutation. However, future study should focus 
on cytosolic and luminal $\mathrm{Ca}^{2+}$ activation as well as the activation and termination of store overload-triggered $\mathrm{Ca}^{2+}$ release in CPVTc-CMs.

By combining calcium sensitivity testing with single channel recordings and $\left[{ }^{3} \mathrm{H}\right]$ ryanodine binding technologies in human embryonic kidney 293 cells or HL-1 CMs overexpressed with Ryr2 heterozygous mutations, the Chen's group has dedicated to investigating of Ryr2 function for several decades. The SOICR mechanism is suggested as a universal mechanism for explaining CPVT pathology. Despite contentious results reported, the highly conserved luminal $\mathrm{Ca}^{2+}$-sensing RYR2 residue E4872, which is located in the transmembrane domain, was identified (Chen et al., 2014). The Chen's group found a novel mutant RYR2 ${ }^{\mathrm{A} 4860 \mathrm{G}}$, which can result in the loss of luminal $\mathrm{Ca}^{2+}$ activation in $\mathrm{Ryr} 2$ and is associated with ventricular fibrillation and sudden death (Jiang et al., 2007). Furthermore, in 2015, Zhao et al. reported a novel loss-offunction mechanism to explain lethal arrhythmia in mice harboring CPVT-linked Ryr2 mutation A4860G (Zhao et al., 2015). They showed that the Ryr2 channel activity was dramatically inhibited, as inferred by $\left[{ }^{3} \mathrm{H}\right]$ ryanodine binding and single channel recordings.

The mutation RYR2 ${ }^{\mathrm{H} 4742 \mathrm{Y}}$ studied in this study has never been investigated before, which is located in the S4 transmembrane segment of the RYR2 channel (residues 4486-4968) (Figure 4), referring to the latest Ryr2 structure. All the S6 segment mutations D4868A, E4872A, R4874A, and E4878A, which were reported previously, showed loss-of-function properties such as decreasing caffeine sensitivities (Peng et al., 2016). Furthermore, suppression of Ryr2 function has been reported recently (Zhong et al., 2016), using a unique mouse model harboring a Rry2 $2^{\mathrm{E} 4872 \mathrm{Q}}$ mutation. A novel RYR2 loss-of-function mutation (I4855M) associated with left ventricular non-compaction and atypical CPVT has also been reported (Roston et al., 2016).

In this study, the beating frequency in CPVTd-CMs with RYR2 $2^{\mathrm{H} 4742 \mathrm{Y}}$ is significantly lower than in Ctrl-CMs. These data are in line with the findings showing that mice heterozygous for the Ryr2 ${ }^{\mathrm{A} 4860 \mathrm{G}}$ mutation (Ryr2-A4860G ${ }^{+/-}$), which is a loss-of-function mutation, exhibited basal bradycardia (Zhao et al., 2015). In ISO-stimulated ventricular CMs, the Ryr2 ${ }^{\mathrm{A} 4860 \mathrm{G}}$ mutation decreased the peak of $\mathrm{Ca}^{2+}$ release during systole, gradually overloading the SR with $\mathrm{Ca}^{2+}$. The resultant $\mathrm{Ca}^{2+}$ overload then randomly caused bursts of prolonged $\mathrm{Ca}^{2+}$ release, activating electrogenic $\mathrm{Na}^{+}-\mathrm{Ca}^{2+}$ exchanger activity and triggering EADs (Zhao et al., 2015). In this study, CPVTd-CMs with the RYR2 ${ }^{\mathrm{H} 4742 \mathrm{Y}}$ mutation showed the increased number of EADs, EAD- 
induced TAs and PVC-like or VT-like events when compared to Ctrl-CMs. It is interesting to observe that the arrhythmic parameters in CPVTd-CMs (number of DADs and TAs, number of EADs and TAs) did not change significantly, but the percentage of arrhythmic cells increased after $100 \mathrm{nM}$ ISO treatment. Furthermore, ISO stimulation of CPVTd-CMs (RYR2 ${ }^{\mathrm{H} 4742 \mathrm{Y}}$ ) leads to increased spontaneous $\mathrm{Ca}^{2+}$ release (L. Cyganek, unpublished data), however, when compared to Ctrl-CMs, the frequency of $\mathrm{Ca}^{2+}$ sparks in CPVTd-CMs after the ISO treatment is significantly lower. These findings suggest that $\mathrm{H} 4742 \mathrm{Y}$ is a loss-of-function mutation. Further studies should be performed to analyze cytosolic and luminal calcium sensitivity, calcium store and caffeine sensitivity in CPVTd-CMs. This is the first study to widen the loss-of-function of RYR2 mutations to the S4 segment of the transmembrane domain.

Based on the advantages of patient-specific iPSC-CMs, it is possible to systematically compare CPVT(a, b, c, d)-CMs harboring RYR2 mutations R420W, A2254V, E4076K, and H4742Y, respectively, with Ctrl-CMs in non-ISO-treated or ISO-challenged conditions. The results showed in this study strongly imply mutation-specific mechanisms in CPVT pathology.

In the non-ISO-stimulated and ISO-stimulated conditions, the beating frequencies of CPVTaCMs and CPVTd-CMs are significantly lower than Ctrl-CMs (Figure 24, Figure 27). This is probably due to the prolonged APDs in CPVTa-CMs and CPVTd-CMs when compared to CtrlCMs (Figure 23).

All the beating frequencies in Ctrl-CMs and CPVT(a, b, c, d)-CMs increased after $100 \mathrm{nM}$ ISO stimulation as assessed by MEA recording (Figure 30B), while the beating frequencies by patchclamp recording showed a more complex profile (Figure 29B). The reasonable explanation is that the frequencies in MEA recordings were calculated from the sinus rhythm electrode, which did not have arrhythmic events (every culture has nine-electrode recordings). However, for patch-clamp recording, the beating frequencies are quantified by AP traces, which contained many arrhythmic events: DADs, EADs, and DADs- or EADs- induced TAs. The not-increased beating frequency in CPVTb- and CPVTc-CMs after the ISO treatment is due to the strongly induced irregular beating, including the stopped beating.

After $100 \mathrm{nM}$ ISO treatment, the parameters assessing the arrhythmic severity degrees, including the percentage of arrhythmic single cells, the percentage of arrhythmic monolayer cultures, number of DADs and TAs, number of PVC-like and VT-like events, and duration of VT-like 
events were significantly higher in $\operatorname{CPVT}(a, b, c)-C M s$ not only in comparison to ISOchallenged Ctrl-CMs but also to their corresponding non-ISO-treated conditions. These results are consistent with previous findings using iPSCs from CPVT patients harboring RYR2 mutations Glu2311Asp (Di Pasquale et al., 2013), F2483I (Fatima et al., 2011b), M4109R (Itzhaki et al., 2012), S406L (Jung et al., 2012), and P2328S (Kujala et al., 2012).

Taken together, the electrophysiological and calcium handling studies demonstrate that iPSCCMs not only can recapitulate CPVT phenotype (DADs, EADs, and TAs in patch-clamp experiments, PVC-like and VT-like events in MEA recording, and altered calcium handling) but also can be used to investigate mutation-specific arrhythmogenesis or to test drugs.

\subsection{Drug testing using CPVT-CMs}

With the advent of iPSC technology, they have become a wonderful tool for modeling and investigating human diseases, for testing new drugs as well as for testing candidate drugs for their cardiotoxicity (Matsa et al., 2014).

Recently, lots of novel and fashionable methods are invented to carry out drug testing or screening, four of which are methods of integrated video microscopy and image analysis software platform to characterize the beating of iPSC-CMs (Maddah et al., 2015), methods using genetically encoded voltage and calcium fluorescent indicators (Shinnawi et al., 2015), methods combining machine learning paired with brightfield optical flow to automate the detection of CM drug effects (Lee et al., 2015) and methods virally introducing optogenetic drivers highthroughput all-optical dynamic cardiac electrophysiology (Klimas et al., 2016). Electrophysiological assessments with patch-clamp and MEA techniques are still golden standard although patch-clamp analysis requires manual operation by a trained electrophysiologist and inherently low-throughput will not be suitable for large-scale drug testing. Liang et al. recently applied whole-cell patch-clamp technique to investigate APD and druginduced arrhythmia and recapitulated drug-induced cardiotoxicity profiles for healthy subjects and patients with long QT syndrome, HCM, and DCM by using disease-specific hiPSC-CMs (Liang et al., 2013). 
Currently, $\beta$-blockers are the basis of antiarrhythmic therapy for CPVT patients (Napolitano et al., 1993). However, for unknown reasons, $\beta$-blockers are unsuccessful in eliminating stressinduced ventricular arrhythmias in approximately 25\% of CPVT patients (Preininger et al., 2016; Roston et al., 2015). Two kinds of clinical surgeries, ICD and left cardiac sympathetic denervation, have many drawbacks. Moreover, some antiarrhythmic drugs (e.g., quinidine, and verapamil), which typically target plasma membrane ion channels, have limited clinical success and in some cases, have been described as being pro-arrhythmic (McCauley and Wehrens, 2011). The pathological release of $\mathrm{Ca}^{2+}$ from the SR through RYR2 could represent a promising target for antiarrhythmic therapy. In this study, four of antiarrhythmic agents (flecainide, dantrolene, rycal1 and rycal2), which mainly target RYR2, are recruited to assess their antiarrhythmic efficacy in CPVT-iPSC-CMs.

In this study, the evaluation of arrhythmic events in Ctrl-CMs and CPVT(a, b, c, d)-CMs in both non-ISO-challenged and ISO-stimulated condition by patch-clamp and MEA techniques demonstrates that CPVT-iPSC-CMs provide a reliable platform for testing of antiarrhythmic drugs and that patch-clamp and MEA techniques are suitable for studying the effect of the drugs on the function of the diseased CMs.

\subsubsection{Two clinical drugs (flecainide and dantrolene) show their antiarrhythmic effects on CPVT(a, b, c)-CMs but not on CPVTd-CMs}

Flecainide is a trifluoroethoxybenzamide that was discovered to be a potent antiarrhythmic agent (McCauley and Wehrens, 2011). The mechanism of therapeutic efficacy of flecainide for CPVT is unclear. Flecainide has a known sodium channel blocking effect (Watanabe et al., 2009). However, Yang et al. recently demonstrated that the effects of flecainide on the $\mathrm{Na}^{+}$channel are insufficient to explain its efficacy in CPVT (Yang et al., 2016). On the other hand, the mechanism suggesting inhibitions of Ryr2 by flecainide (Hilliard et al., 2010; Watanabe et al., 2009) is becoming controversial (Bannister et al., 2015).

Watanabe et al. reported that flecainide prevents CPVT in mice by decreasing Ryr2 opening probability (Watanabe et al., 2009). The authors also indicated that flecainide completely prevented CPVT in two human subjects who had remained highly symptomatic on conventional 
drug therapy. Liu et al. demonstrated that flecainide had an antiarrhythmic effect in a CPVT mouse Ryr2 ${ }^{\mathrm{R} 4496 \mathrm{C}}$ model by increasing the threshold for triggered activity through its sodium channel blocking effect (Liu et al., 2011). In recent years, Itzhaki et al. showed that flecainide completely eliminated or significantly decreased afterdepolarizations and triggered activities in patient-specific CPVT (M4109R) -hiPSCs-CMs (Itzhaki et al., 2012).

In this study, systematic comparison of the antiarrhythmic efficacy of flecainide in CPVT-CMs with different mutation-specific mutations in RYR2 was performed for the first time.

Flecainide showed antiarrhythmic effects on $\operatorname{CPVT}(\mathrm{a}, \mathrm{b}$, c)-CMs harboring RYR2 mutations R420W, A2254V, and E4076K, demonstrated by electrophysiological results (Figure 31, Figure 32). These findings are consistent with the previously published data using iPSC-CMs from a CPVT patient carrying the RYR2 ${ }^{\mathrm{M} 4109 \mathrm{R}}$ mutation (Itzhaki et al., 2012), which is located in the central domain (residues 3613-4207) of RYR2. However, flecainide had no antiarrhythmic effect on CPVTd-CMs with the RYR2 ${ }^{\mathrm{H} 4742 \mathrm{Y}}$ mutation, and even a slightly increased number of EADs or EAD-induced TAs was observed in CPVTd-CMs after treatment with flecainide. It is evidently suggesting that physicians should consider the CPVT patients' genetic background when flecainide is planned for clinical treatment of the disease.

Dantrolene is a specific agent for the treatment of malignant hyperthermia. It is known that dantrolene can inhibit $\mathrm{Ca}^{2+}$ leak by correcting the defective inter-domain interaction between $\mathrm{N}$ terminal (1-619 amino acid) and central (2,000-2,500 amino acid) domains of RYRs (both RYR1 and RYR2) (Kobayashi et al., 2009). Dantrolene has been shown to improve intracellular $\mathrm{Ca}^{2+}$ handling in failing CMs from a canine model of heart failure and prevent arrhythmias in a knock-in Ryr2 ${ }^{\mathrm{R} 2474 \mathrm{~S}}$ mouse model of CPVT (Kobayashi et al., 2009). It has been demonstrated that superfusion of CPVT (S406L) hiPSCs-CMs with $10 \mu \mathrm{M}$ dantrolene could prevent the appearance of arrhythmia (Jung et al., 2012).

Dantrolene reduce the number of PVCs on average of 74\% (range 33-97) in four patients with Nterminal or central mutations in the cytosolic region of the RYR2 protein, while dantrolene had no effect in two patients with mutations in Q4201R or near the transmembrane domain (V4653F) (Penttinen et al., 2015). This was the first publication to show some clues for different mechanisms of arrhythmia pathogenesis related to the mutation-specific RYR2 mutations, 
however only one drug (dantrolene) was investigated for its antiarrhythmic effect on various RYR2 mutations.

In this study, dantrolene showed antiarrhythmic effects on CPVT(a, b, c)-CMs harboring RYR2 mutations R420W, A2254V and E4076K but not on CPVTd-CMs with the RYR2 mutation H4742Y, shown by electrophysiology results (Figure 33, Figure 34), which is consistent with the data from Penttinen et al. (Penttinen et al., 2015).

Future studies should focus on the mechanisms how flecainide and dantrolene affect the function of CPVT-CMs.

\subsubsection{Two experimental RYR2 stabilizers (rycal1 and rycal2) show their antiarrhythmic effects on CPVT(a, b, c)-CMs but not on CPVTd-CMs}

The classic fight-or-flight stress response involves activation of the sympathetic nervous system and release of catecholamines that bind to $\beta$-ARs and raise the second messenger cAMP levels, which activates PKA (Eschenhagen, 2010). PKA activates RYR2, the LTCC and the calcium uptake pathway through the calcium pump. When PKA phosphorylates RYR2 in the heart, calstabin2 is dissociated, increasing the activity of the channel (Brillantes et al., 1994; Jayaraman et al., 1992; Marx et al., 2000; Wehrens et al., 2003).

In 1994, The Marks's group reported that Ryr expressed without calstabin in insects resulted in hyperactive leaky calcium channels, nevertheless, the channel was stabilized and the leak was "fixed" when Ryr was co-expressed with calstabin (Brillantes et al., 1994). Calstabin2 deficiency is a critical mediator of triggers that initiate cardiac arrhythmias, which were confirmed in calstabin2-deficient mice and with enhancing calstabin2 binding to Ryrs by JTV519 (1,4benzothiazepinea) (Lehnart et al., 2006; Wehrens et al., 2005; Wehrens et al., 2004b). JTV519 was designed as one of the rycals, which are small-molecule therapeutic drugs developed first in the laboratory of Dr. Andrew R. Marks at Columbia University in New York, which inhibit the Ryr2 leak by enhancing calstabin2 binding to the RYR2 channel and stabilizing its closed state (Wehrens et al., 2004b). JTV519 can effectively reduce arrhythmic events triggered by elevation of $\mathrm{SR} \mathrm{Ca}^{2+}$ load of murine ventricular myocytes harboring a human $\mathrm{RYR} 2^{\mathrm{R} 4496 \mathrm{C}+/}$ - mutation (Sedej et al., 2010). Treatment of phosphorylated Ryr2-P2328S channels with JTV519 
completely normalized the gain-of-function defect, and the significant leftward shift of $\mathrm{Ca}^{2+}$ sensitivity was rescued (Lehnart et al., 2004). However, the mechanism of action of JTV519 is complex and conflicting. It was reported that JTV519 suppressed spontaneous $\mathrm{Ca}^{2+}$ release and inhibited $\left[{ }^{3} \mathrm{H}\right]$ ryanodine binding to Ryr2 irrespective of FKBP12.6 association (Hunt et al., 2007). Recently, this drug was reported to be a $\mathrm{Ca}^{2+}$-dependent blocker of sarco/endoplasmic reticulum $\mathrm{Ca}^{2+}$-ATPase and partial agonist of RYRs (Darcy et al., 2016).

Generally, rycals are designed to restore the normal function of RYR2 channels and are not RYR2 channel blockers. In this study, two candidate drugs for the CPVT treatment, rycal1 and rycal2, were provided by a pharmacological company.

Rycal1 (Figure 35, Figure 36) and rycal2 (Figure 37, Figure 38) demonstrated antiarrhythmic effects on CPVT(a, b, c)-CMs harboring mutations R420W, A2254V, E4076K, respectively, but not on CPVTd-CMs with RYR2 mutation H4742Y.

Taken together, CMs derived from CPVT-iPSCs display mutation-specific arrhythmogenesis features, and these profiles have a profound impact on antiarrhythmic drugs' treatment. Moreover, the application of two electrophysiological methods exhibits a good example for studying drug effects in combination with iPSC technology.

\subsection{Differentially regulated cAMP dynamics in Ctrl-CMs and CPVT-CMs}

Due to the lack of in vitro specimen, the PDE activities have not been well investigated in human CMs (Mehel et al., 2013; Molina et al., 2012). To investigate if the cAMP levels are altered in cytosol or at the RYR2 microdomain in CPVT-CMs, FRET measurements were performed in Epac1-camps- or Epac1-camps-JNC expressing CMs, respectively. No existing empirical research addresses $\beta$-adrenergic signaling during the CPVT pathological remodeling by using iPSC-CMs. The results showed that even low concentration of ISO can induce FRET change in Epac1-camps- or Epac1-camps-JNC-expressing iPSC-CMs despite some immature features of iPSC-CMs (Figure 39).

The FRET change in Epac1-camps-expressing CPVTa-CMs was significantly higher when compared to Ctrl-CMs, indicating that the cytosolic cAMP level is higher in CPVTa-CMs than in Ctrl-CMs after $10 \mathrm{nM}$ ISO treatment. Moreover, the FRET change in Epac1-camps-JNC- 
expressing CPVTb- and CPVTc-CMs was significantly higher when compared to Ctrl-CMs, indicating that the cAMP levels at the RYR2 microdomain are higher in these CPVT-CMs than in Ctrl-CMs after $10 \mathrm{nM}$ ISO treatment. Interestingly, the FRET change in Epac1-camps-JNCexpressing CPVTd-CMs was significantly lower when compared to Ctrl-CMs, indicating that the cAMP level at the RYR2 microdomain is lower in CPVTd-CMs than in Ctrl-CMs after $10 \mathrm{nM}$ ISO treatment. These data may imply that the altered PKA activities in different CPVT-iPSCCMs with different RYR2 mutations. Currently, RYR2 has three well-defined phosphorylation sites, Ser2814, Ser2030 and Ser2808 although additional putative phosphorylation sites at Ser2810, Ser2811, Ser2797 and Thr2876 have been reported (Yuchi et al., 2012). Ser2030 and Ser2808 are mainly phosphorylated by PKA (Marx et al., 2000; Xiao et al., 2006). However, in our lab, high baseline phosphorylation of the RYR2 residue Ser2808 was found in both Ctrl- and CPVT(a, b, c)-CMs; and no patient-specific differences of the basal phosphorylation status was observed in CPVT(a, b, c)-CMs when compared to Ctrl-CMs (Henze, 2016). After ISO stimulation, phosphorylation of Ser2808 was slightly increased in CPVT-CMs and Ctrl-CMs, but mutation- or patient-specific differences were not observed (Henze, 2016), which might be due to the high baseline phosphorylation of the RYR2 residue Ser2808. Future study should investigate the phosphorylation status of the other RYR2 residues, which are mainly phosphorylated by PKA in CPVT(a, b, c)-CMs, and analyze the phosphorylation status of the RYR2 residues Ser2808 and Ser2030 in CPVTd-CMs.

\subsubsection{PDE2 and PDE3 activities are decreased in CPVT-CMs}

Abnormalities of $\beta$-adrenergic signaling have been well documented in DCM (Ahmet et al., 2008; Lowes et al., 2002). Recently, expression profiles and functional assays confirmed a significant upregulation of PDE subtypes PDE 2A and 3A in DCM iPSC-CMs (Wu et al., 2015). The PDE3A subfamily is proposed to be the most important cAMP-degrading PDE in human CMs. Interestingly, a recent report showed that PDE2 was markedly up-regulated in human failing hearts and desensitized $\beta$-AR against acute $\beta$-AR stimulation by using human and/or canine experimental heart failure (Mehel et al., 2013). There are several publications reported the decreasing of PDE3 activity and PDE3 protein levels in left ventricular endocardium in pacinginduced failing canine hearts (Smith et al., 1998). 
There is little known about the relationship between the CPVT and the activities of PDE2 and PDE3. This is the first study to investigate the activities of PDE2 and PDE3 in CPVT-iPSC-CMs. The results revealed that the activities of PDE2 and PDE3 to degradate cAMP decreased no matter in cytosol or at the RYR2 compartment in CPVT(a, b, c, d)-CMs harboring mutations R420W, A2254V, E4076K and H4742Y, respecitively. Future studies should investigate expression levels of PDE2 and PDE3 in $\operatorname{CPVT}(\mathrm{a}, \mathrm{b}, \mathrm{c}, \mathrm{d})-\mathrm{CM}$ at both RNA and protein levels.

\subsubsection{PDE4 activity shows mutation-specific properties in CPVT-CMs}

It was reported that PDE4 inhibition not only increased intracellular cAMP and L-type $\mathrm{Ca}^{2+}$ current and dramatically delayed their decay after a short adrenergic stimulation, but also increased the frequency of $\mathrm{Ca}^{2+}$ sparks at baseline, as well as the contractile response and the incidence of arrhythmias in human atrial strips during $\beta$-adrenergic stimulation (Molina et al., 2012). Previous study reported that PDE4 partially protected the right ventricle against $\beta$ adrenaline evoked arrhythmias (Galindo-Tovar and Kaumann, 2008).

In this study, when iPSC-CMs were treated with $10 \mathrm{nM}$ ISO, the inhibition of PDE4 caused big increases in cAMP levels that saturated both Epac1-camps and Epac1-camps-JNC sensors. Therefore, PDE4 inhibition was repeated after the treatment with $1 \mathrm{nM}$ ISO to measure the dynamic range of the FRET sensors. These data indicate that PDE4 is the major regulator of cAMP level in iPSC-CMs after ISO stimulation and that PDE2 and PDE3 have a smaller contribution to regulate the cAMP level in cytosol and at the RYR2 compartment. Similar data have been reported in ventricular CMs after ISO stimulation, which were isolated from mice expressing Epac1-camps or Epac1-camps-JNC sensors (Götz, 2014).

It was demonstrated that PDE4D3 and other key components of cAMP signaling (PKA and phosphatases) were associated with RYR2 via mAKAP and formed a cAMP microdomain that regulated RYR2 (Dodge et al., 2001; Lehnart, 2007; Lehnart et al., 2005). Furthermore, it was postulated that during heart failure, depletion of PDE4D3 from the microdomain led to PKAmediated hyperphosphorylation of RYR2 at Ser2808 and associated $\mathrm{Ca}^{2+}$ leak (Lehnart et al., 2005). 
In this study, CPVT(b, c)-CMs, but not CPVT(a, d)-CMs, showed reduced activities of PDE4 in cytosol (Figure 44). However, the phosphorylation of RYR2 Ser2808 was not altered in CPVT(b, c)-iPSC-CMs after ISO stimulation (Henze, 2016). This could be explained by the previous suggestion that PKA is fully active, even at basal and unstimulated state (Beavo and Brunton, 2002).

It was reported that in adult ventricular CMs, global or basal cAMP level is $\sim 1.2 \mu \mathrm{M}$ (Iancu et al., 2008), whereas the affinity of PKA is in the range of 10-100 nM in vitro. Further study is needed to figure out whether the phosphorylation status of other proteins important for calcium handling is altered in CPVT-CMs. In addition, it is necessary to analyze which PDE4 isoforms participate in mutation-specific CPVT arrhythmogenesis.

Based on the FRET measurements in Epac1-camps-JNC, the RYR2 compartmental PDE4 activity is reduced in CPVT(c, d)-CMs but not in CPVT(a, b)-CMs after ISO stimulation, when compared to Ctrl-CMs. However, the function of PDE4 in CPVT(c, d)-CMs is still unclear. It was hypothesized that local PDE4D3 activity might shape a pool of low cAMP concentrations at the cardiac RYR to protect the channel from excessive phosphorylation (Götz, 2014). In CtrlCMs, $\beta$-adrenergic stimulation causes an increase in cAMP, strong enough to overcome the PDE4D3 barrier, to activate PKA for channel phosphorylation and $\mathrm{Ca}^{2+}$ gating to facilitate myocyte contraction. However, for CPVT-CMs, mutations in RYR2 may cause PDE4D3 depletion, hyperphosphorylation and leaky RyR2 (increased $\mathrm{Ca}^{2+}$ gating during systole or diastole), which are associated with arrhythmias. As mentioned above, further study is needed to figure out whether the phosphorylation status of other proteins important for calcium handling is altered in CPVT-CMs, how different PDEs play roles in arrhythmogenesis of CPVT, and whether the expression levels or locations are altered in CPVT-CMs.

To my knowledge, this is the first research that focus on cAMP dynamic in CPVT pathology by combining the technologies of iPSC disease modeling and FRET. It implies that there are probably some connections between the altered activities of PDE2, 3, 4 and the distinguish arrhythmogenesis of CPVT caused by different mutation-specific mutations. 


\subsection{Future perspectives}

Although these results strongly imply a domain- or mutation-specific pathophysiological mechanism in CPVT(a, b, c, d)-CMs harboring different RYR2 mutations, the calcium handling experiments (cytosolic and luminal calcium sensitivity of RYR2, SR calcium contents, the threshold of SOICR, etc.) should be applied to further figure out the exact differences in underlying mechanism between the non-transmembrane domain and the transmembrane domain mutation-caused CPVT.

A powerful gene-editing technology of clustered regularly interspaced short palindromic repeats is the biggest game changer since PCR. It gives an opportunity to systematical and reliable disease modeling in relevant human cell types by a combination of iPSCs and gene editing technologies (Soldner and Jaenisch, 2012). It is worthy to use the powerful gene-editing tool to correct the specific RYR2 point mutations to figure out whether the disease phenotypes observed in this study are mutation-specific.

Recently, epigenetic regulation has been shown to be related to HCM, DCM, and diabetic cardiomyopathy (Asrih and Steffens, 2013; Haas et al., 2013; Wu et al., 2015). It is a top priority to perform gene and protein expression profiling and to study epigenetic changes of PDE2, PDE3, and PDE4, which may help to figure out genetic CPVT pathological remodeling in the future.

The potential strategy of direct conversion of human fibroblasts into functional CMs by small molecules would shorten the timeline for disease modeling.

Since the complexities of the RYR2 channel and its binding proteins, domain-specific mechanisms are not enough to summarize all the mutations. In the personalized medicine era, the mutation-specific mechanisms should be investigated in order to precisely design correspondent therapies. 


\section{References}

Abi-Gerges, A., Richter, W., Lefebvre, F., Mateo, P., Varin, A., Heymes, C., Samuel, J.L., Lugnier, C., Conti, M., Fischmeister, R., et al. (2009). Decreased expression and activity of cAMP phosphodiesterases in cardiac hypertrophy and its impact on beta-adrenergic cAMP signals. Circulation research 105, 784-792.

Ahmet, I., Krawczyk, M., Zhu, W., Woo, A.Y.-H., Morrell, C., Poosala, S., Xiao, R.-p., Lakatta, E.G., and Talan, M.I. (2008). Cardioprotective and Survival Benefits of Long-Term Combined Therapy with $\beta 2$ Adrenoreceptor (AR) Agonist and $\beta 1$ AR Blocker in Dilated Cardiomyopathy Postmyocardial Infarction. Journal of Pharmacology and Experimental Therapeutics 325, 491499.

Amit, M., and Itskovitz-Eldor, J. (2002). Derivation and spontaneous differentiation of human embryonic stem cells*. Journal of Anatomy 200, 225-232.

Asrih, M., and Steffens, S. (2013). Emerging role of epigenetics and miRNA in diabetic cardiomyopathy. Cardiovasc Pathol 22, 117-125.

Aye, T.T., Soni, S., van Veen, T.A., van der Heyden, M.A., Cappadona, S., Varro, A., de Weger, R.A., de Jonge, N., Vos, M.A., Heck, A.J., et al. (2012). Reorganized PKA-AKAP associations in the failing human heart. Journal of molecular and cellular cardiology 52, 511-518.

Baim , D.S., McDowell , A.V., Cherniles , J., Monrad , E.S., Parker , J.A., Edelson , J., Braunwald , E., and Grossman , W. (1983). Evaluation of a New Bipyridine Inotropic Agent Milrinone - in Patients with Severe Congestive Heart Failure. New England Journal of Medicine 309, 748-756.

Bannister, M.L., Thomas, N.L., Sikkel, M.B., Mukherjee, S., Maxwell, C., MacLeod, K.T., George, C.H., and Williams, A.J. (2015). The Mechanism of Flecainide Action in CPVT Does Not Involve a Direct Effect on RyR2. Circulation research 116, 1324-1335.

Beavo, J.A., and Brunton, L.L. (2002). Cyclic nucleotide research [mdash] still expanding after half a century. Nature reviews Molecular cell biology 3, 710-718.

Bers, D.M. (2002). Cardiac excitation-contraction coupling. Nature 415, 198-205.

Bhuiyan, Z.A., Hamdan, M.A., Shamsi, E.T., Postma, A.V., Mannens, M.M., Wilde, A.A., and Al-Gazali, L. (2007). A novel early onset lethal form of catecholaminergic polymorphic ventricular tachycardia maps to chromosome 7p14-p22. Journal of cardiovascular electrophysiology 18, 1060-1066.

Blayney, L.M., and Lai, F.A. (2009). Ryanodine receptor-mediated arrhythmias and sudden cardiac death. Pharmacol Ther 123, 151-177.

Borko, L., Bauerova-Hlinkova, V., Hostinova, E., Gasperik, J., Beck, K., Lai, F.A., Zahradnikova, A., and Sevcik, J. (2014). Structural insights into the human RyR2 N-terminal region involved in cardiac arrhythmias. Acta Crystallogr D Biol Crystallogr 70, 2897-2912. Boularan, C., and Gales, C. (2015). Cardiac cAMP: production, hydrolysis, modulation and detection. Front Pharmacol 6, 203.

Brillantes, A.-M.B., Ondrias, K., Scott, A., Kobrinsky, E., Ondriašová, E., Moschella, M.C., Jayaraman, T., Landers, M., Ehrlich, B.E., and Marks, A.R. (1994). Stabilization of calcium release channel (ryanodine receptor) function by FK506-binding protein. Cell 77, 513-523. Brodde, O.E. (1991). Beta 1- and beta 2-adrenoceptors in the human heart: properties, function, and alterations in chronic heart failure. Pharmacological Reviews 43, 203-242. 
Brooker, G., Harper, J.F., Terasaki, W.L., and Moylan, R.D. (1979). Radioimmunoassay of cyclic AMP and cyclic GMP. Advances in cyclic nucleotide research 10, 1-33.

Burridge, P.W., Matsa, E., Shukla, P., Lin, Z.C., Churko, J.M., Ebert, A.D., Lan, F., Diecke, S., Huber, B., Mordwinkin, N.M., et al. (2014). Chemically defined generation of human cardiomyocytes. Nature methods.

Buta, C., David, R., Dressel, R., Emgard, M., Fuchs, C., Gross, U., Healy, L., Hescheler, J., Kolar, R., Martin, U., et al. (2013). Reconsidering pluripotency tests: do we still need teratoma assays? Stem cell research 11, 552-562.

Calebiro, D., Nikolaev, V.O., Gagliani, M.C., de Filippis, T., Dees, C., Tacchetti, C., Persani, L., and Lohse, M.J. (2009). Persistent cAMP-signals triggered by internalized G-protein-coupled receptors. PLoS Biol 7, e1000172.

Cao, N., Huang, Y., Zheng, J., Spencer, C.I., Zhang, Y., Fu, J.-D., Nie, B., Xie, M., Zhang, M., Wang, H., et al. (2016). Conversion of human fibroblasts into functional cardiomyocytes by small molecules. Science 352, 1216-1220.

Cerrone, M., Colombi, B., Santoro, M., di Barletta, M.R., Scelsi, M., Villani, L., Napolitano, C., and Priori, S.G. (2005). Bidirectional ventricular tachycardia and fibrillation elicited in a knockin mouse model carrier of a mutation in the cardiac ryanodine receptor. Circulation research 96 , e77-82.

Cerrone, M., Noujaim, S.F., Tolkacheva, E.G., Talkachou, A., O'Connell, R., Berenfeld, O., Anumonwo, J., Pandit, S.V., Vikstrom, K., Napolitano, C., et al. (2007). Arrhythmogenic mechanisms in a mouse model of catecholaminergic polymorphic ventricular tachycardia. Circulation research 101, 1039-1048.

Chen, W., Wang, R., Chen, B., Zhong, X., Kong, H., Bai, Y., Zhou, Q., Xie, C., Zhang, J., Guo, A., et al. (2014). The ryanodine receptor store-sensing gate controls $\mathrm{Ca} 2+$ waves and $\mathrm{Ca} 2+-$ triggered arrhythmias. Nature medicine 20, 184-192.

Chuva de Sousa Lopes, S.M., Hassink, R.J., Feijen, A., van Rooijen, M.A., Doevendans, P.A., Tertoolen, L., Brutel de la Riviere, A., and Mummery, C.L. (2006). Patterning the heart, a template for human cardiomyocyte development. Dev Dyn 235, 1994-2002.

Curtis, M.J., Hancox, J.C., Farkas, A., Wainwright, C.L., Stables, C.L., Saint, D.A., ClementsJewery, H., Lambiase, P.D., Billman, G.E., Janse, M.J., et al. (2013). The Lambeth Conventions (II): guidelines for the study of animal and human ventricular and supraventricular arrhythmias. Pharmacol Ther 139, 213-248.

Darcy, Y.L., Diaz-Sylvester, P.L., and Copello, J.A. (2016). K201 (JTV519) is a Ca2+Dependent Blocker of SERCA and a Partial Agonist of Ryanodine Receptors in Striated Muscle. Mol Pharmacol 90, 106-115.

Di Pasquale, E., Lodola, F., Miragoli, M., Denegri, M., Avelino-Cruz, J.E., Buonocore, M., Nakahama, H., Portararo, P., Bloise, R., Napolitano, C., et al. (2013). CaMKII inhibition rectifies arrhythmic phenotype in a patient-specific model of catecholaminergic polymorphic ventricular tachycardia. Cell death \& disease 4, e843.

Ding, B., Abe, J., Wei, H., Huang, Q., Walsh, R.A., Molina, C.A., Zhao, A., Sadoshima, J., Blaxall, B.C., Berk, B.C., et al. (2005). Functional role of phosphodiesterase 3 in cardiomyocyte apoptosis: implication in heart failure. Circulation 111, 2469-2476.

DiPilato, L.M., Cheng, X., and Zhang, J. (2004). Fluorescent indicators of cAMP and Epac activation reveal differential dynamics of cAMP signaling within discrete subcellular compartments. Proceedings of the National Academy of Sciences of the United States of America 101, 16513-16518. 
Dittrich, M., Jurevičius, J., Georget, M., Rochais, F., Fleischmann, B.K., Hescheler, J., and Fischmeister, R. (2001). Local response of L-type $\mathrm{Ca}(2+)$ current to nitric oxide in frog ventricular myocytes. The Journal of Physiology 534, 109-121.

Dobrev, D., Carlsson, L., and Nattel, S. (2012). Novel molecular targets for atrial fibrillation therapy. Nat Rev Drug Discov 11, 275-291.

Dodge-Kafka, K.L., Soughayer, J., Pare, G.C., Carlisle Michel, J.J., Langeberg, L.K., Kapiloff, M.S., and Scott, J.D. (2005). The protein kinase A anchoring protein mAKAP coordinates two integrated cAMP effector pathways. Nature 437, 574-578.

Dodge, K.L., Khouangsathiene, S., Kapiloff, M.S., Mouton, R., Hill, E.V., Houslay, M.D., Langeberg, L.K., and Scott, J.D. (2001). mAKAP assembles a protein kinase A/PDE4 phosphodiesterase cAMP signaling module. The EMBO Journal 20, 1921-1930.

Domingo, D., Neco, P., Fernandez-Pons, E., Zissimopoulos, S., Molina, P., Olague, J., SuarezMier, M.P., Lai, F.A., Gomez, A.M., and Zorio, E. (2015). Non-ventricular, Clinical, and Functional Features of the RyR2(R420Q) Mutation Causing Catecholaminergic Polymorphic Ventricular Tachycardia. Rev Esp Cardiol (Engl Ed) 68, 398-407.

Ebert, A.D., Yu, J., Rose, F.F., Jr., Mattis, V.B., Lorson, C.L., Thomson, J.A., and Svendsen, C.N. (2009). Induced pluripotent stem cells from a spinal muscular atrophy patient. Nature 457, 277-280.

Egashira, T., Yuasa, S., Suzuki, T., Aizawa, Y., Yamakawa, H., Matsuhashi, T., Ohno, Y., Tohyama, S., Okata, S., Seki, T., et al. (2012). Disease characterization using LQTS-specific induced pluripotent stem cells. Cardiovascular research 95, 419-429.

Eschenhagen, T. (2010). Is ryanodine receptor phosphorylation key to the fight or flight response and heart failure? The Journal of clinical investigation 120, 4197-4203.

Evans, M.J., and Kaufman, M.H. (1981). Establishment in culture of pluripotential cells from mouse embryos. Nature 292, 154-156.

Fatima, A., Xu, G., Shao, K., Papadopoulos, S., Lehmann, M., Arnaiz-Cot, J.J., Rosa, A.O., Nguemo, F., Matzkies, M., Dittmann, S., et al. (2011a). In vitro modeling of ryanodine receptor 2 dysfunction using human induced pluripotent stem cells. Cellular physiology and biochemistry : international journal of experimental cellular physiology, biochemistry, and pharmacology 28 , 579-592.

Fatima, A., Xu, G., Shao, K., Papadopoulos, S., Lehmann, M., Arnáiz-Cot, J.J., Rosa, A.O., Nguemo, F., Matzkies, M., Dittmann, S., et al. (2011b). <i>In vitro</i> Modeling of Ryanodine Receptor 2 Dysfunction Using Human Induced Pluripotent Stem Cells. Cellular Physiology and Biochemistry 28, 579-592.

Fischmeister, R., Castro, L.R., Abi-Gerges, A., Rochais, F., Jurevicius, J., Leroy, J., and Vandecasteele, G. (2006). Compartmentation of cyclic nucleotide signaling in the heart: the role of cyclic nucleotide phosphodiesterases. Circulation research 99, 816-828.

Förster, T. (1948). Zwischenmolekulare Energiewanderung und Fluoreszenz. Annalen der Physik 437, 55-75.

Friedrichs, S., Malan, D., Voss, Y., and Sasse, P. (2015). Scalable Electrophysiological Investigation of iPS Cell-Derived Cardiomyocytes Obtained by a Lentiviral Purification Strategy. Journal of Clinical Medicine 4, 102-123.

Fujie, Y., Fusaki, N., Katayama, T., Hamasaki, M., Soejima, Y., Soga, M., Ban, H., Hasegawa, M., Yamashita, S., Kimura, S., et al. (2014). New type of Sendai virus vector provides transgene-free iPS cells derived from chimpanzee blood. PLoS One 9, e113052. 
Fusaki, N., Ban, H., Nishiyama, A., Saeki, K., and Hasegawa, M. (2009). Efficient induction of transgene-free human pluripotent stem cells using a vector based on Sendai virus, an RNA virus that does not integrate into the host genome. Proceedings of the Japan Academy, Series B 85, 348-362.

Galende, E., Karakikes, I., Edelmann, L., Desnick, R.J., Kerenyi, T., Khoueiry, G., Lafferty, J., McGinn, J.T., Brodman, M., Fuster, V., et al. (2010). Amniotic fluid cells are more efficiently reprogrammed to pluripotency than adult cells. Cellular reprogramming 12, 117-125.

Galindo-Tovar, A., and Kaumann, A.J. (2008). Phosphodiesterase-4 blunts inotropism and arrhythmias but not sinoatrial tachycardia of (-)-adrenaline mediated through mouse cardiac beta(1)-adrenoceptors. Br J Pharmacol 153, 710-720.

Götz, K. (2014). Real time visualization of cGMP and cAMP dynamics in intact adult cardiomyocytes using new transgenic mice.

Gramlich, M., Pane, L.S., Zhou, Q., Chen, Z., Murgia, M., Schotterl, S., Goedel, A., Metzger, K., Brade, T., Parrotta, E., et al. (2015). Antisense-mediated exon skipping: a therapeutic strategy for titin-based dilated cardiomyopathy. EMBO molecular medicine 7, 562-576.

Guo, W., Sun, B., Xiao, Z., Liu, Y., Wang, Y., Zhang, L., Wang, R., and Chen, S.R. (2016). The EF-hand Ca2+ Binding Domain Is Not Required for Cytosolic Ca2+ Activation of the Cardiac Ryanodine Receptor. The Journal of biological chemistry 291, 2150-2160.

Haas, J., Frese, K.S., Park, Y.J., Keller, A., Vogel, B., Lindroth, A.M., Weichenhan, D., Franke, J., Fischer, S., Bauer, A., et al. (2013). Alterations in cardiac DNA methylation in human dilated cardiomyopathy. EMBO molecular medicine 5, 413-429.

Hentze, H., Soong, P.L., Wang, S.T., Phillips, B.W., Putti, T.C., and Dunn, N.R. (2009).

Teratoma formation by human embryonic stem cells: evaluation of essential parameters for future safety studies. Stem cell research 2, 198-210.

Henze, S. (2016). Induced pluripotent stem cell-derived cardiomyocytes as model for studying CPVT caused by mutations in RYR2.

Hilliard, F.A., Steele, D.S., Laver, D., Yang, Z., Le Marchand, S.J., Chopra, N., Piston, D.W., Huke, S., and Knollmann, B.C. (2010). Flecainide inhibits arrhythmogenic Ca2+ waves by open state block of ryanodine receptor $\mathrm{Ca} 2+$ release channels and reduction of $\mathrm{Ca} 2+$ spark mass. Journal of molecular and cellular cardiology 48, 293-301.

Horackova, M., and Byczko, Z. (1997). Differences in the structural characteristics of adult guinea pig and rat cardiomyocytes during their adaptation and maintenance in long-term cultures: confocal microscopy study. Experimental cell research 237, 158-175.

Hou, P., Li, Y., Zhang, X., Liu, C., Guan, J., Li, H., Zhao, T., Ye, J., Yang, W., Liu, K., et al. (2013). Pluripotent Stem Cells Induced from Mouse Somatic Cells by Small-Molecule Compounds. Science 341, 651-654.

Hunt, D.J., Jones, P.P., Wang, R., Chen, W., Bolstad, J., Chen, K., Shimoni, Y., and Chen, S.R. (2007). K201 (JTV519) suppresses spontaneous Ca2+ release and [3H]ryanodine binding to RyR2 irrespective of FKBP12.6 association. Biochem J 404, 431-438.

lancu, R.V., Ramamurthy, G., Warrier, S., Nikolaev, V.O., Lohse, M.J., Jones, S.W., and Harvey, R.D. (2008). Cytoplasmic cAMP concentrations in intact cardiac myocytes, Vol 295.

Ieda, M., Fu, J.D., Delgado-Olguin, P., Vedantham, V., Hayashi, Y., Bruneau, B.G., and Srivastava, D. (2010). Direct reprogramming of fibroblasts into functional cardiomyocytes by defined factors. Cell 142, 375-386.

Imbrogno, S., Gattuso, A., Mazza, R., Angelone, T., and Cerra, M.C. (2015). beta3 -AR and the vertebrate heart: a comparative view. Acta Physiol (Oxf) 214, 158-175. 
Itskovitz-Eldor, J., Schuldiner, M., Karsenti, D., Eden, A., Yanuka, O., Amit, M., Soreq, H., and Benvenisty, N. (2000). Differentiation of human embryonic stem cells into embryoid bodies compromising the three embryonic germ layers. Molecular Medicine 6, 88-95.

Itzhaki, I., Maizels, L., Huber, I., Gepstein, A., Arbel, G., Caspi, O., Miller, L., Belhassen, B., Nof, E., Glikson, M., et al. (2012). Modeling of catecholaminergic polymorphic ventricular tachycardia with patient-specific human-induced pluripotent stem cells. Journal of the American College of Cardiology 60, 990-1000.

Itzhaki, I., Maizels, L., Huber, I., Zwi-Dantsis, L., Caspi, O., Winterstern, A., Feldman, O., Gepstein, A., Arbel, G., Hammerman, H., et al. (2011). Modelling the long QT syndrome with induced pluripotent stem cells. Nature 471, 225-229.

Jaenisch, R., and Young, R. (2008). Stem cells, the molecular circuitry of pluripotency and nuclear reprogramming. Cell 132, 567-582.

Jayaraman, T., Brillantes, A.M., Timerman, A.P., Fleischer, S., Erdjument-Bromage, H., Tempst, P., and Marks, A.R. (1992). FK506 binding protein associated with the calcium release channel (ryanodine receptor). Journal of Biological Chemistry 267, 9474-9477.

Jiang, D., Chen, W., Wang, R., Zhang, L., and Chen, S.R. (2007). Loss of luminal Ca2+ activation in the cardiac ryanodine receptor is associated with ventricular fibrillation and sudden death. Proceedings of the National Academy of Sciences of the United States of America 104, 18309-18314.

Jiang, D., Xiao, B., Yang, D., Wang, R., Choi, P., Zhang, L., Cheng, H., and Chen, S.R. (2004). RyR2 mutations linked to ventricular tachycardia and sudden death reduce the threshold for store-overload-induced Ca2+ release (SOICR). Proceedings of the National Academy of Sciences of the United States of America 101, 13062-13067.

Jung, C.B., Moretti, A., Mederos y Schnitzler, M., Iop, L., Storch, U., Bellin, M., Dorn, T., Ruppenthal, S., Pfeiffer, S., Goedel, A., et al. (2012). Dantrolene rescues arrhythmogenic RYR2 defect in a patient-specific stem cell model of catecholaminergic polymorphic ventricular tachycardia. EMBO molecular medicine 4, 180-191.

Kane, C., Couch, L., and Terracciano, C.M.N. (2015). Excitation-contraction coupling of human induced pluripotent stem cell-derived cardiomyocytes. Frontiers in Cell and Developmental Biology 3.

Kannankeril, P.J., Mitchell, B.M., Goonasekera, S.A., Chelu, M.G., Zhang, W., Sood, S., Kearney, D.L., Danila, C.I., De Biasi, M., Wehrens, X.H., et al. (2006). Mice with the R176Q cardiac ryanodine receptor mutation exhibit catecholamine-induced ventricular tachycardia and cardiomyopathy. Proceedings of the National Academy of Sciences of the United States of America 103, 12179-12184.

Kashimura, T., Briston, S.J., Trafford, A.W., Napolitano, C., Priori, S.G., Eisner, D.A., and Venetucci, L.A. (2010). In the RyR2(R4496C) mouse model of CPVT, beta-adrenergic stimulation induces $\mathrm{Ca}$ waves by increasing SR Ca content and not by decreasing the threshold for Ca waves. Circulation research 107, 1483-1489.

Kim, D., Kim, C.H., Moon, J.I., Chung, Y.G., Chang, M.Y., Han, B.S., Ko, S., Yang, E., Cha, K.Y., Lanza, R., et al. (2009). Generation of human induced pluripotent stem cells by direct delivery of reprogramming proteins. Cell stem cell 4, 472-476.

Kimlicka, L., Tung, C.C., Carlsson, A.C., Lobo, P.A., Yuchi, Z., and Van Petegem, F. (2013). The cardiac ryanodine receptor $\mathrm{N}$-terminal region contains an anion binding site that is targeted by disease mutations. Structure 21, 1440-1449. 
Kirshenbaum, L.A., MacLellan, W.R., Mazur, W., French, B.A., and Schneider, M.D. (1993). Highly efficient gene transfer into adult ventricular myocytes by recombinant adenovirus. The Journal of clinical investigation 92, 381-387.

Klimas, A., Ambrosi, C.M., Yu, J., Williams, J.C., Bien, H., and Entcheva, E. (2016).

OptoDyCE as an automated system for high-throughput all-optical dynamic cardiac electrophysiology. Nat Commun 7, 11542.

Kobayashi, S., Yano, M., Suetomi, T., Ono, M., Tateishi, H., Mochizuki, M., Xu, X., Uchinoumi, H., Okuda, S., Yamamoto, T., et al. (2009). Dantrolene, a therapeutic agent for malignant hyperthermia, markedly improves the function of failing cardiomyocytes by stabilizing interdomain interactions within the ryanodine receptor. Journal of the American College of Cardiology 53, 1993-2005.

Kobayashi, S., Yano, M., Uchinoumi, H., Suetomi, T., Susa, T., Ono, M., Xu, X., Tateishi, H., Oda, T., Okuda, S., et al. (2010). Dantrolene, a Therapeutic Agent for Malignant Hyperthermia, Inhibits Catecholaminergic Polymorphic Ventricular Tachycardia in a RyR2R2474S/+ Knock-In Mouse Model. Circulation Journal 74, 2579-2584.

Kontula, K., Laitinen, P.J., Lehtonen, A., Toivonen, L., Viitasalo, M., and Swan, H. (2005). Catecholaminergic polymorphic ventricular tachycardia: Recent mechanistic insights. Cardiovascular research 67, 379-387.

Kujala, K., Paavola, J., Lahti, A., Larsson, K., Pekkanen-Mattila, M., Viitasalo, M., Lahtinen, A.M., Toivonen, L., Kontula, K., Swan, H., et al. (2012). Cell model of catecholaminergic polymorphic ventricular tachycardia reveals early and delayed afterdepolarizations. PLoS One 7 , e44660.

Kushnir, A., and Marks, A.R. (2010). The Ryanodine Receptor in Cardiac Physiology and Disease. In Advances in Pharmacology, M.V. Paul, ed. (Academic Press), pp. 1-30.

Lahat, H., Pras, E., Olender, T., Avidan, N., Ben-Asher, E., Man, O., Levy-Nissenbaum, E., Khoury, A., Lorber, A., Goldman, B., et al. (2001). A missense mutation in a highly conserved region of CASQ2 is associated with autosomal recessive catecholamine-induced polymorphic ventricular tachycardia in Bedouin families from Israel. Am J Hum Genet 69, 1378-1384. Lahti, A.L., Kujala, V.J., Chapman, H., Koivisto, A.P., Pekkanen-Mattila, M., Kerkela, E., Hyttinen, J., Kontula, K., Swan, H., Conklin, B.R., et al. (2012). Model for long QT syndrome type 2 using human iPS cells demonstrates arrhythmogenic characteristics in cell culture.

Disease models \& mechanisms 5, 220-230.

Lan, F., Lee, A.S., Liang, P., Sanchez-Freire, V., Nguyen, P.K., Wang, L., Han, L., Yen, M., Wang, Y.M., Sun, N., et al. (2013). Abnormal Calcium Handling Properties Underlie Familial Hypertrophic Cardiomyopathy Pathology in Patient-Specific Induced Pluripotent Stem Cells. Cell stem cell 12, 101-113.

Lee, E.K., Kurokawa, Y.K., Tu, R., George, S.C., and Khine, M. (2015). Machine learning plus optical flow: a simple and sensitive method to detect cardioactive drugs. Scientific reports 5, 11817.

Leenhardt, A., Denjoy, I., and Guicheney, P. (2012). Catecholaminergic polymorphic ventricular tachycardia. Circ Arrhythm Electrophysiol 5, 1044-1052.

Leenhardt, A., Lucet, V., Denjoy, I., Grau, F., Ngoc, D.D., and Coumel, P. (1995).

Catecholaminergic polymorphic ventricular tachycardia in children. A 7-year follow-up of 21 patients. Circulation 91, 1512-1519.

Lehnart, S.E. (2007). Novel targets for treating heart and muscle disease: stabilizing ryanodine receptors and preventing intracellular calcium leak. Curr Opin Pharmacol 7, 225-232. 
Lehnart, S.E., Mongillo, M., Bellinger, A., Lindegger, N., Chen, B.X., Hsueh, W., Reiken, S., Wronska, A., Drew, L.J., Ward, C.W., et al. (2008). Leaky Ca2+ release channel/ryanodine receptor 2 causes seizures and sudden cardiac death in mice. The Journal of clinical investigation $118,2230-2245$.

Lehnart, S.E., Terrenoire, C., Reiken, S., Wehrens, X.H., Song, L.S., Tillman, E.J., Mancarella, S., Coromilas, J., Lederer, W.J., Kass, R.S., et al. (2006). Stabilization of cardiac ryanodine receptor prevents intracellular calcium leak and arrhythmias. Proceedings of the National Academy of Sciences of the United States of America 103, 7906-7910.

Lehnart, S.E., Wehrens, X.H., Laitinen, P.J., Reiken, S.R., Deng, S.X., Cheng, Z., Landry, D.W., Kontula, K., Swan, H., and Marks, A.R. (2004). Sudden death in familial polymorphic ventricular tachycardia associated with calcium release channel (ryanodine receptor) leak. Circulation 109, 3208-3214.

Lehnart, S.E., Wehrens, X.H., Reiken, S., Warrier, S., Belevych, A.E., Harvey, R.D., Richter, W., Jin, S.L., Conti, M., and Marks, A.R. (2005). Phosphodiesterase 4D deficiency in the ryanodinereceptor complex promotes heart failure and arrhythmias. Cell 123, 25-35.

Leroy, J., Richter, W., Mika, D., Castro, L.R., Abi-Gerges, A., Xie, M., Scheitrum, C., Lefebvre, F., Schittl, J., Mateo, P., et al. (2011). Phosphodiesterase 4B in the cardiac L-type Ca(2)(+) channel complex regulates $\mathrm{Ca}(2)(+)$ current and protects against ventricular arrhythmias in mice. The Journal of clinical investigation 121, 2651-2661.

Lian, X., Hsiao, C., Wilson, G., Zhu, K., Hazeltine, L.B., Azarin, S.M., Raval, K.K., Zhang, J., Kamp, T.J., and Palecek, S.P. (2012). Robust cardiomyocyte differentiation from human pluripotent stem cells via temporal modulation of canonical Wnt signaling. Proceedings of the National Academy of Sciences of the United States of America 109, E1848-1857.

Lian, X., Zhang, J., Azarin, S.M., Zhu, K., Hazeltine, L.B., Bao, X., Hsiao, C., Kamp, T.J., and Palecek, S.P. (2013). Directed cardiomyocyte differentiation from human pluripotent stem cells by modulating Wnt/beta-catenin signaling under fully defined conditions. Nature protocols 8 , 162-175.

Liang, P., Lan, F., Lee, A.S., Gong, T., Sanchez-Freire, V., Wang, Y., Diecke, S., Sallam, K., Knowles, J.W., Wang, P.J., et al. (2013). Drug screening using a library of human induced pluripotent stem cell-derived cardiomyocytes reveals disease-specific patterns of cardiotoxicity. Circulation 127, 1677-1691.

Lieve, K.V., van der Werf, C., and Wilde, A.A. (2016). Catecholaminergic Polymorphic Ventricular Tachycardia. Circ J 80, 1285-1291.

Lin, T., Ambasudhan, R., Yuan, X., Li, W., Hilcove, S., Abujarour, R., Lin, X., Hahm, H.S., Hao, E., Hayek, A., et al. (2009). A chemical platform for improved induction of human iPSCs. Nature methods 6, 805-808.

Liu, N., Denegri, M., Ruan, Y., Avelino-Cruz, J.E., Perissi, A., Negri, S., Napolitano, C., Coetzee, W.A., Boyden, P.A., and Priori, S.G. (2011). Short communication: flecainide exerts an antiarrhythmic effect in a mouse model of catecholaminergic polymorphic ventricular tachycardia by increasing the threshold for triggered activity. Circulation research 109, 291-295. Liu, N., Rizzi, N., Boveri, L., and Priori, S.G. (2009). Ryanodine receptor and calsequestrin in arrhythmogenesis: what we have learnt from genetic diseases and transgenic mice. Journal of molecular and cellular cardiology 46, 149-159.

Liu, Z., Wang, R., Tian, X., Zhong, X., Gangopadhyay, J., Cole, R., Ikemoto, N., Chen, S.R., and Wagenknecht, T. (2010). Dynamic, inter-subunit interactions between the N-terminal and central mutation regions of cardiac ryanodine receptor. J Cell Sci 123, 1775-1784. 
Lowes , B.D., Gilbert , E.M., Abraham , W.T., Minobe , W.A., Larrabee , P., Ferguson , D., Wolfel , E.E., Lindenfeld , J., Tsvetkova , T., Robertson , A.D., et al. (2002). Myocardial Gene Expression in Dilated Cardiomyopathy Treated with Beta-Blocking Agents. New England Journal of Medicine 346, 1357-1365.

Maddah, M., Heidmann, J.D., Mandegar, M.A., Walker, C.D., Bolouki, S., Conklin, B.R., and Loewke, K.E. (2015). A non-invasive platform for functional characterization of stem-cellderived cardiomyocytes with applications in cardiotoxicity testing. Stem Cell Reports 4, 621-631. Malan, D., Friedrichs, S., Fleischmann, B.K., and Sasse, P. (2011). Cardiomyocytes obtained from induced pluripotent stem cells with long-QT syndrome 3 recapitulate typical diseasespecific features in vitro. Circulation research 109, 841-847.

Marks, A.R. (2013). Calcium cycling proteins and heart failure: mechanisms and therapeutics. The Journal of clinical investigation 123, 46-52.

Marx, S.O., Reiken, S., Hisamatsu, Y., Jayaraman, T., Burkhoff, D., Rosemblit, N., and Marks, A.R. (2000). PKA Phosphorylation Dissociates FKBP12.6 from the Calcium Release Channel (Ryanodine Receptor): Defective Regulation in Failing Hearts. Cell 101, 365-376.

Matsa, E., Burridge, P.W., and Wu, J.C. (2014). Human Stem Cells for Modeling Heart Disease and for Drug Discovery. Sci Transl Med 6, 239ps236.

Matsa, E., Rajamohan, D., Dick, E., Young, L., Mellor, I., Staniforth, A., and Denning, C. (2011). Drug evaluation in cardiomyocytes derived from human induced pluripotent stem cells carrying a long QT syndrome type 2 mutation. European heart journal 32, 952-962.

McCauley, M.D., and Wehrens, X.H. (2011). Targeting ryanodine receptors for anti-arrhythmic therapy. Acta Pharmacol Sin 32, 749-757.

Medeiros-Domingo, A., Bhuiyan, Z.A., Tester, D.J., Hofman, N., Bikker, H., van Tintelen, J.P., Mannens, M.M., Wilde, A.A., and Ackerman, M.J. (2009). The RYR2-encoded ryanodine receptor/calcium release channel in patients diagnosed previously with either catecholaminergic polymorphic ventricular tachycardia or genotype negative, exercise-induced long QT syndrome: a comprehensive open reading frame mutational analysis. Journal of the American College of Cardiology 54, 2065-2074.

Mehel, H., Emons, J., Vettel, C., Wittkopper, K., Seppelt, D., Dewenter, M., Lutz, S., Sossalla, S., Maier, L.S., Lechene, P., et al. (2013). Phosphodiesterase-2 is up-regulated in human failing hearts and blunts beta-adrenergic responses in cardiomyocytes. Journal of the American College of Cardiology 62, 1596-1606.

Mika, D., Richter, W., Westenbroek, R.E., Catterall, W.A., and Conti, M. (2014). PDE4B mediates local feedback regulation of beta(1)-adrenergic cAMP signaling in a sarcolemmal compartment of cardiac myocytes. J Cell Sci 127, 1033-1042.

Miller, C.L., and Yan, C. (2010). Targeting Cyclic Nucleotide Phosphodiesterase in the Heart: Therapeutic Implications. Journal of cardiovascular translational research 3, 507-515. Mohler, P.J., and Wehrens, X.H.T. (2007). Mechanisms of Human Arrhythmia Syndromes: Abnormal Cardiac Macromolecular Interactions. Physiology 22, 342-350.

Molina, C.E., Leroy, J., Richter, W., Xie, M., Scheitrum, C., Lee, I.O., Maack, C., RuckerMartin, C., Donzeau-Gouge, P., Verde, I., et al. (2012). Cyclic adenosine monophosphate phosphodiesterase type 4 protects against atrial arrhythmias. Journal of the American College of Cardiology 59, 2182-2190.

Mongillo, M., Tocchetti, C.G., Terrin, A., Lissandron, V., Cheung, Y.F., Dostmann, W.R., Pozzan, T., Kass, D.A., Paolocci, N., Houslay, M.D., et al. (2006). Compartmentalized 
phosphodiesterase-2 activity blunts beta-adrenergic cardiac inotropy via an NO/cGMPdependent pathway. Circulation research 98, 226-234.

Moretti , A., Bellin , M., Welling , A., Jung , C.B., Lam , J.T., Bott-Flügel , L., Dorn , T., Goedel , A., Höhnke , C., Hofmann , F., et al. (2010). Patient-Specific Induced Pluripotent StemCell Models for Long-QT Syndrome. New England Journal of Medicine 363, 1397-1409. Napolitano, C., Priori, S.G., and Bloise, R. (1993). Catecholaminergic Polymorphic Ventricular Tachycardia. In GeneReviews(R), R.A. Pagon, M.P. Adam, H.H. Ardinger, S.E. Wallace, A. Amemiya, L.J.H. Bean, T.D. Bird, C.T. Fong, H.C. Mefford, R.J.H. Smith, et al., eds. (Seattle (WA): University of Washington, Seattle

University of Washington, Seattle. All rights reserved.).

Napolitano., C., Priori., S.G., and Bloise., R. (2014). Catecholaminergic Polymorphic Ventricular Tachycardia.

Nikolaev, V.O., Bunemann, M., Hein, L., Hannawacker, A., and Lohse, M.J. (2004). Novel single chain cAMP sensors for receptor-induced signal propagation. The Journal of biological chemistry 279, 37215-37218.

Nikolaev, V.O., Bunemann, M., Schmitteckert, E., Lohse, M.J., and Engelhardt, S. (2006). Cyclic AMP imaging in adult cardiac myocytes reveals far-reaching beta1-adrenergic but locally confined beta2-adrenergic receptor-mediated signaling. Circulation research 99, 1084-1091. Nikolaev, V.O., and Lohse, M.J. (2006). Monitoring of cAMP synthesis and degradation in living cells. Physiology 21, 86-92.

Nikolaev, V.O., Moshkov, A., Lyon, A.R., Miragoli, M., Novak, P., Paur, H., Lohse, M.J., Korchev, Y.E., Harding, S.E., and Gorelik, J. (2010). $\beta 2$-Adrenergic Receptor Redistribution in Heart Failure Changes cAMP Compartmentation. Science 327, 1653-1657.

Nishino, K., Toyoda, M., Yamazaki-Inoue, M., Fukawatase, Y., Chikazawa, E., Sakaguchi, H., Akutsu, H., and Umezawa, A. (2011). DNA Methylation Dynamics in Human Induced Pluripotent Stem Cells over Time. PLOS Genetics 7, e1002085.

Nishio, H., Iwata, M., and Suzuki, K. (2006). Postmortem molecular screening for cardiac ryanodine receptor type 2 mutations in sudden unexplained death: R420W mutated case with characteristics of status thymico-lymphatics. Circ J 70, 1402-1406.

Nishio, H., Okudaira, N., Matsushita, K., Yoshimoto, T., Sato, T., and Suzuki, K. (2014). Hypertrophy of lymphoid organs is a possible phenotypic characteristic of R420W mutation of the cardiac ryanodine receptor gene: a study using a knock-in mouse model. Leg Med (Tokyo) $16,326-332$.

Novak, A., Barad, L., Lorber, A., Gherghiceanu, M., Reiter, I., Eisen, B., Eldor, L., ItskovitzEldor, J., Eldar, M., Arad, M., et al. (2015). Functional abnormalities in iPSC-derived cardiomyocytes generated from CPVT1 and CPVT2 patients carrying ryanodine or calsequestrin mutations. Journal of cellular and molecular medicine, n/a-n/a.

Novak, A., Barad, L., Zeevi-Levin, N., Shick, R., Shtrichman, R., Lorber, A., Itskovitz-Eldor, J., and Binah, O. (2012). Cardiomyocytes generated from CPVTD307H patients are arrhythmogenic in response to beta-adrenergic stimulation. Journal of cellular and molecular medicine 16, 468-482.

Nyegaard, M., Overgaard, Michael T., Søndergaard, Mads T., Vranas, M., Behr, Elijah R., Hildebrandt, Lasse L., Lund, J., Hedley, Paula L., Camm, A.J., Wettrell, G., et al. (2012). Mutations in Calmodulin Cause Ventricular Tachycardia and Sudden Cardiac Death. The American Journal of Human Genetics 91, 703-712. 
Okita, K., Matsumura, Y., Sato, Y., Okada, A., Morizane, A., Okamoto, S., Hong, H., Nakagawa, M., Tanabe, K., Tezuka, K., et al. (2011). A more efficient method to generate integration-free human iPS cells. Nature methods 8, 409-412.

Okudaira, N., Kuwahara, M., Hirata, Y., Oku, Y., and Nishio, H. (2014). A knock-in mouse model of N-terminal R420W mutation of cardiac ryanodine receptor exhibits arrhythmogenesis with abnormal calcium dynamics in cardiomyocytes. Biochem Biophys Res Commun 452, 665668.

Omori, K., and Kotera, J. (2007). Overview of PDEs and their regulation. Circulation research 100, 309-327.

Packer, M. (1989). Effect of phosphodiesterase inhibitors on survival of patients with chronic congestive heart failure. The American journal of cardiology 63, 41a-45a.

Packer, M., Carver, J.R., Rodeheffer , R.J., Ivanhoe , R.J., DiBianco , R., Zeldis , S.M., Hendrix , G.H., Bommer, W.J., Elkayam , U., Kukin , M.L., et al. (1991). Effect of Oral Milrinone on Mortality in Severe Chronic Heart Failure. New England Journal of Medicine 325, 1468-1475.

Pallante, B.A., Giovannone, S., Fang-Yu, L., Zhang, J., Liu, N., Kang, G., Dun, W., Boyden, P.A., and Fishman, G.I. (2010). Contactin-2 expression in the cardiac Purkinje fiber network. Circ Arrhythm Electrophysiol 3, 186-194.

Peng, W., Shen, H., Wu, J., Guo, W., Pan, X., Wang, R., Chen, S.R.W., and Yan, N. (2016). Structural basis for the gating mechanism of the type 2 ryanodine receptor RyR2. Science.

Penttinen, K., Swan, H., Vanninen, S., Paavola, J., Lahtinen, A.M., Kontula, K., and Aalto-Setala, K. (2015). Antiarrhythmic Effects of Dantrolene in Patients with Catecholaminergic Polymorphic Ventricular Tachycardia and Replication of the Responses Using iPSC Models. PLoS One 10, e0125366.

Ponsioen, B., Zhao, J., Riedl, J., Zwartkruis, F., van der Krogt, G., Zaccolo, M., Moolenaar, W.H., Bos, J.L., and Jalink, K. (2004). Detecting cAMP-induced Epac activation by fluorescence resonance energy transfer: Epac as a novel cAMP indicator. EMBO reports 5, 1176-1180. Postma, A.V., Denjoy, I., Kamblock, J., Alders, M., Lupoglazoff, J.M., Vaksmann, G., DubosqBidot, L., Sebillon, P., Mannens, M.M., Guicheney, P., et al. (2005). Catecholaminergic polymorphic ventricular tachycardia: RYR2 mutations, bradycardia, and follow up of the patients. J Med Genet 42, 863-870.

Preininger, M.K., Jha, R., Maxwell, J.T., Wu, Q., Singh, M., Wang, B., Dalal, A., McEachin, Z.T., Rossoll, W., Hales, C.M., et al. (2016). A human pluripotent stem cell model of catecholaminergic polymorphic ventricular tachycardia recapitulates patient-specific drug responses. Disease models \& mechanisms.

Priori, S.G. (2002). Clinical and Molecular Characterization of Patients With Catecholaminergic Polymorphic Ventricular Tachycardia. Circulation 106, 69-74.

Priori, S.G., and Chen, S.R. (2011). Inherited dysfunction of sarcoplasmic reticulum $\mathrm{Ca} 2+$ handling and arrhythmogenesis. Circulation research 108, 871-883.

Priori, S.G., and Napolitano, C. (2005). Cardiac and skeletal muscle disorders caused by mutations in the intracellular $\mathrm{Ca} 2+$ release channels. The Journal of clinical investigation 115 , 2033-2038.

Priori, S.G., Napolitano, C., Memmi, M., Colombi, B., Drago, F., Gasparini, M., DeSimone, L., Coltorti, F., Bloise, R., Keegan, R., et al. (2002). Clinical and molecular characterization of patients with catecholaminergic polymorphic ventricular tachycardia. Circulation 106, 69-74. 
Priori, S.G., Napolitano, C., Tiso, N., Memmi, M., Vignati, G., Bloise, R., Sorrentino, V., and Danieli, G.A. (2001). Mutations in the Cardiac Ryanodine Receptor Gene (hRyR2) Underlie Catecholaminergic Polymorphic Ventricular Tachycardia. Circulation 103, 196-200.

Priori, S.G., Wilde, A.A., Horie, M., Cho, Y., Behr, E.R., Berul, C., Blom, N., Brugada, J., Chiang, C.E., Huikuri, H., et al. (2013). Executive summary: HRS/EHRA/APHRS expert consensus statement on the diagnosis and management of patients with inherited primary arrhythmia syndromes. Europace 15, 1389-1406.

Richter, W., Xie, M., Scheitrum, C., Krall, J., Movsesian, M.A., and Conti, M. (2011). Conserved expression and functions of PDE4 in rodent and human heart. Basic Research in Cardiology 106, 249-262.

Rivet-Bastide, M., Vandecasteele, G., Hatem, S., Verde, I., Bénardeau, A., Mercadier, J.J., and Fischmeister, R. (1997). cGMP-stimulated cyclic nucleotide phosphodiesterase regulates the basal calcium current in human atrial myocytes. Journal of Clinical Investigation 99, 2710-2718. Roston, T.M., Guo, W., Krahn, A.D., Wang, R., van Petegem, F., Sanatani, S., Chen, S.R.W., and Lehman, A. (2016). A Novel RYR2 Loss-of-Function Mutation (I4855M) is Associated with Left Ventricular Non-compaction and Atypical Catecholaminergic Polymorphic Ventricular Tachycardia. Journal of electrocardiology.

Roston, T.M., Vinocur, J.M., Maginot, K.R., Mohammed, S., Salerno, J.C., Etheridge, S.P., Cohen, M., Hamilton, R.M., Pflaumer, A., Kanter, R.J., et al. (2015). Catecholaminergic polymorphic ventricular tachycardia in children: analysis of therapeutic strategies and outcomes from an international multicenter registry. Circ Arrhythm Electrophysiol 8, 633-642.

Roux-Buisson, N., Cacheux, M., Fourest-Lieuvin, A., Fauconnier, J., Brocard, J., Denjoy, I., Durand, P., Guicheney, P., Kyndt, F., Leenhardt, A., et al. (2012). Absence of triadin, a protein of the calcium release complex, is responsible for cardiac arrhythmia with sudden death in human. Human molecular genetics 21, 2759-2767.

Sato, N., Asai, K., Okumura, S., Takagi, G., Shannon, R.P., Fujita-Yamaguchi, Y., Ishikawa, Y., Vatner, S.F., and Vatner, D.E. (1999). Mechanisms of desensitization to a PDE inhibitor (milrinone) in conscious dogs with heart failure. The American journal of physiology 276, H1699-1705.

Scott, J.D., and Santana, L.F. (2010). A-kinase anchoring proteins: getting to the heart of the matter. Circulation 121, 1264-1271.

Sedej, S., Heinzel, F.R., Walther, S., Dybkova, N., Wakula, P., Groborz, J., Gronau, P., Maier, L.S., Vos, M.A., Lai, F.A., et al. (2010). Na+-dependent SR Ca2+ overload induces arrhythmogenic events in mouse cardiomyocytes with a human CPVT mutation. Cardiovascular research 87, 50-59.

Shi, Y., Desponts, C., Do, J.T., Hahm, H.S., Scholer, H.R., and Ding, S. (2008). Induction of pluripotent stem cells from mouse embryonic fibroblasts by Oct4 and Klf4 with small-molecule compounds. Cell stem cell 3, 568-574.

Shinnawi, R., Huber, I., Maizels, L., Shaheen, N., Gepstein, A., Arbel, G., Tijsen, A.J., and Gepstein, L. (2015). Monitoring Human-Induced Pluripotent Stem Cell-Derived Cardiomyocytes with Genetically Encoded Calcium and Voltage Fluorescent Reporters. Stem Cell Reports. Smith, C.J., He, J., Ricketts, S.G., Ding, J.-Z., Moggio, R.A., and Hintze, T.H. (1998). Downregulation of right ventricular phosphodiesterase PDE-3A mRNA and protein before the development of canine heart failure. Cell Biochemistry and Biophysics 29, 67-88.

Smith, C.J., Huang, R., Sun, D., Ricketts, S., Hoegler, C., Ding, J.Z., Moggio, R.A., and Hintze, T.H. (1997). Development of decompensated dilated cardiomyopathy is associated with 
decreased gene expression and activity of the milrinone-sensitive cAMP phosphodiesterase PDE3A. Circulation 96, 3116-3123.

Sogo, T., Morikawa, K., Kurata, Y., Li, P., Ichinose, T., Yuasa, S., Nozaki, D., Miake, J., Ninomiya, H., Shimizu, W., et al. (2016). Electrophysiological properties of iPS cell-derived cardiomyocytes from a patient with long QT syndrome type 1 harboring the novel mutation M437V of KCNQ1. Regenerative Therapy 4, 9-17.

Soldner, F., and Jaenisch, R. (2012). iPSC Disease Modeling. Science 338, 1155-1156.

Song, L., Alcalai, R., Arad, M., Wolf, C.M., Toka, O., Conner, D.A., Berul, C.I., Eldar, M., Seidman, C.E., and Seidman, J.G. (2007). Calsequestrin 2 (CASQ2) mutations increase expression of calreticulin and ryanodine receptors, causing catecholaminergic polymorphic ventricular tachycardia. The Journal of clinical investigation 117, 1814-1823.

Stadtfeld, M., Apostolou, E., Akutsu, H., Fukuda, A., Follett, P., Natesan, S., Kono, T., Shioda, T., and Hochedlinger, K. (2010). Aberrant silencing of imprinted genes on chromosome 12qF1 in mouse induced pluripotent stem cells. Nature 465, 175-181.

Stadtfeld, M., Nagaya, M., Utikal, J., Weir, G., and Hochedlinger, K. (2008). Induced Pluripotent Stem Cells Generated Without Viral Integration. Science 322, 945-949.

Streckfuss-Bomeke, K., Wolf, F., Azizian, A., Stauske, M., Tiburcy, M., Wagner, S., Hubscher, D., Dressel, R., Chen, S., Jende, J., et al. (2013). Comparative study of human-induced pluripotent stem cells derived from bone marrow cells, hair keratinocytes, and skin fibroblasts. European heart journal 34, 2618-2629.

Suetomi, T., Yano, M., Uchinoumi, H., Fukuda, M., Hino, A., Ono, M., Xu, X., Tateishi, H., Okuda, S., Doi, M., et al. (2011). Mutation-linked defective interdomain interactions within ryanodine receptor cause aberrant $\mathrm{Ca}(2)(+)$ release leading to catecholaminergic polymorphic ventricular tachycardia. Circulation 124, 682-694.

Sumitomo, N. (2015). Current topics in catecholaminergic polymorphic ventricular tachycardia. Journal of Arrhythmia.

Sun, N., Yazawa, M., Liu, J., Han, L., Sanchez-Freire, V., Abilez, O.J., Navarrete, E.G., Hu, S., Wang, L., Lee, A., et al. (2012). Patient-specific induced pluripotent stem cells as a model for familial dilated cardiomyopathy. Sci Transl Med 4, 130 ra147.

Takahashi, K., Osanai, T., Nakano, T., Wakui, M., and Okumura, K. (2002). Enhanced activities and gene expression of phosphodiesterase types 3 and 4 in pressure-induced congestive heart failure. Heart and Vessels 16, 249-256.

Takahashi, K., Tanabe, K., Ohnuki, M., Narita, M., Ichisaka, T., Tomoda, K., and Yamanaka, S. (2007). Induction of pluripotent stem cells from adult human fibroblasts by defined factors. Cell $131,861-872$.

Takahashi, K., and Yamanaka, S. (2006). Induction of pluripotent stem cells from mouse embryonic and adult fibroblast cultures by defined factors. Cell 126, 663-676.

Tamaoki, N., Takahashi, K., Tanaka, T., Ichisaka, T., Aoki, H., Takeda-Kawaguchi, T., Iida, K., Kunisada, T., Shibata, T., Yamanaka, S., et al. (2010). Dental pulp cells for induced pluripotent stem cell banking. J Dent Res 89, 773-778.

Terrenoire, C., Wang, K., Tung, K.W., Chung, W.K., Pass, R.H., Lu, J.T., Jean, J.C., Omari, A., Sampson, K.J., Kotton, D.N., et al. (2013). Induced pluripotent stem cells used to reveal drug actions in a long QT syndrome family with complex genetics. J Gen Physiol 141, 61-72.

Thomson, J.A., Itskovitz-Eldor, J., Shapiro, S.S., Waknitz, M.A., Swiergiel, J.J., Marshall, V.S., and Jones, J.M. (1998). Embryonic Stem Cell Lines Derived from Human Blastocysts. Science $282,1145-1147$. 
Timerman, A.P., Jayaraman, T., Wiederrecht, G., Onoue, H., Marks, A.R., and Fleischer, S. (1994). The Ryanodine Receptor from Canine Heart Sarcoplasmic Reticulum Is Associated with a Novel FK-506 Binding Protein. Biochemical and Biophysical Research Communications 198, 701-706.

Tohyama, S., Hattori, F., Sano, M., Hishiki, T., Nagahata, Y., Matsuura, T., Hashimoto, H., Suzuki, T., Yamashita, H., Satoh, Y., et al. (2013). Distinct metabolic flow enables large-scale purification of mouse and human pluripotent stem cell-derived cardiomyocytes. Cell stem cell 12, 127-137.

van der Werf, C., Nederend, I., Hofman, N., van Geloven, N., Ebink, C., Frohn-Mulder, I.M., Alings, A.M., Bosker, H.A., Bracke, F.A., van den Heuvel, F., et al. (2012a). Familial evaluation in catecholaminergic polymorphic ventricular tachycardia: disease penetrance and expression in cardiac ryanodine receptor mutation-carrying relatives. Circ Arrhythm Electrophysiol 5, 748-756. van der Werf, C., Zwinderman, A.H., and Wilde, A.A. (2012b). Therapeutic approach for patients with catecholaminergic polymorphic ventricular tachycardia: state of the art and future developments. Europace 14, 175-183.

Vaskova, E.A., Stekleneva, A.E., Medvedev, S.P., and Zakian, S.M. (2013). "Epigenetic Memory" Phenomenon in Induced Pluripotent Stem Cells. Acta Naturae 5, 15-21.

Wallukat, G. (2002). The beta-adrenergic receptors. Herz 27, 683-690.

Wang, Y., and Wang, N. (2009). FRET and mechanobiology. Integrative Biology 1, 565-573. Warren, L., Manos, P.D., Ahfeldt, T., Loh, Y.H., Li, H., Lau, F., Ebina, W., Mandal, P.K., Smith, Z.D., Meissner, A., et al. (2010). Highly efficient reprogramming to pluripotency and directed differentiation of human cells with synthetic modified mRNA. Cell stem cell 7, 618-630. Watanabe, H., Chopra, N., Laver, D., Hwang, H.S., Davies, S.S., Roach, D.E., Duff, H.J., Roden, D.M., Wilde, A.A.M., and Knollmann, B.C. (2009). Flecainide prevents catecholaminergic polymorphic ventricular tachycardia in mice and humans. Nature medicine 15, 380-383. Wehrens, X.H., Lehnart, S.E., Reiken, S., van der Nagel, R., Morales, R., Sun, J., Cheng, Z., Deng, S.X., de Windt, L.J., Landry, D.W., et al. (2005). Enhancing calstabin binding to ryanodine receptors improves cardiac and skeletal muscle function in heart failure. Proceedings of the National Academy of Sciences of the United States of America 102, 9607-9612. Wehrens, X.H., Lehnart, S.E., Reiken, S.R., and Marks, A.R. (2004a). Ca2+/calmodulindependent protein kinase II phosphorylation regulates the cardiac ryanodine receptor. Circulation research 94, e61-70.

Wehrens, X.H., and Marks, A.R. (2003). Altered function and regulation of cardiac ryanodine receptors in cardiac disease. Trends in biochemical sciences 28, 671-678.

Wehrens, X.H.T., Lehnart, S.E., Huang, F., Vest, J.A., Reiken, S.R., Mohler, P.J., Sun, J., Guatimosim, S., Song, L.-S., Rosemblit, N., et al. (2003). FKBP12.6 Deficiency and Defective Calcium Release Channel (Ryanodine Receptor) Function Linked to Exercise-Induced Sudden Cardiac Death. Cell 113, 829-840.

Wehrens, X.H.T., Lehnart, S.E., Reiken, S.R., Deng, S.-X., Vest, J.A., Cervantes, D., Coromilas, J., Landry, D.W., and Marks, A.R. (2004b). Protection from Cardiac Arrhythmia Through Ryanodine Receptor-Stabilizing Protein Calstabin2. Science 304, 292-296.

Williams, C. (2004). cAMP detection methods in HTS: selecting the best from the rest. Nat Rev Drug Discov 3, 125-135.

Wu, H., Lee, J., Vincent, L.G., Wang, Q., Gu, M., Lan, F., Churko, J.M., Sallam, K.I., Matsa, E., Sharma, A., et al. (2015). Epigenetic Regulation of Phosphodiesterases 2A and 3A Underlies 
Compromised beta-Adrenergic Signaling in an iPSC Model of Dilated Cardiomyopathy. Cell stem cell 17, 89-100.

$\mathrm{Wu}, \mathrm{P}$., and Brand, L. (1994). Resonance energy transfer: methods and applications. Analytical biochemistry 218, 1-13.

Xiang, Y., Naro, F., Zoudilova, M., Jin, S.L., Conti, M., and Kobilka, B. (2005).

Phosphodiesterase 4D is required for beta2 adrenoceptor subtype-specific signaling in cardiac myocytes. Proceedings of the National Academy of Sciences of the United States of America $102,909-914$.

Xiang, Y.K. (2011). Compartmentalization of beta-adrenergic signals in cardiomyocytes. Circulation research 109, 231-244.

Xiao, B., Zhong, G., Obayashi, M., Yang, D., Chen, K., Walsh, M.P., Shimoni, Y., Cheng, H., Ter Keurs, H., and Chen, S.R. (2006). Ser-2030, but not Ser-2808, is the major phosphorylation site in cardiac ryanodine receptors responding to protein kinase A activation upon betaadrenergic stimulation in normal and failing hearts. Biochem J 396, 7-16.

Xiong, H., Feng, X., Gao, L., Xu, L., Pasek, D.A., Seok, J.H., and Meissner, G. (1998). Identification of a two EF-hand $\mathrm{Ca} 2+$ binding domain in lobster skeletal muscle ryanodine receptor/Ca2+ release channel. Biochemistry 37, 4804-4814.

Xu, Y., Zhu, X., Hahm, H.S., Wei, W., Hao, E., Hayek, A., and Ding, S. (2010). Revealing a core signaling regulatory mechanism for pluripotent stem cell survival and self-renewal by small molecules. Proceedings of the National Academy of Sciences of the United States of America 107, 8129-8134.

Yakubov, E., Rechavi, G., Rozenblatt, S., and Givol, D. (2010). Reprogramming of human fibroblasts to pluripotent stem cells using mRNA of four transcription factors. Biochem Biophys Res Commun 394, 189-193.

Yan, C., Miller, C.L., and Abe, J. (2007). Regulation of phosphodiesterase 3 and inducible cAMP early repressor in the heart. Circulation research 100, 489-501.

Yan, Z., Bai, X.C., Yan, C., Wu, J., Li, Z., Xie, T., Peng, W., Yin, C.C., Li, X., Scheres, S.H., et al. (2015). Structure of the rabbit ryanodine receptor RyR1 at near-atomic resolution. Nature 517, 50-55.

Yanaka, N., Kurosawa, Y., Minami, K., Kawai, E., and Omori, K. (2003). cGMPphosphodiesterase activity is up-regulated in response to pressure overload of rat ventricles. Biosci Biotechnol Biochem 67, 973-979.

Yang, P.C., Moreno, J.D., Miyake, C.Y., Vaughn-Behrens, S.B., Jeng, M.T., Grandi, E., Wehrens, X.H., Noskov, S.Y., and Clancy, C.E. (2016). In silico prediction of drug therapy in catecholaminergic polymorphic ventricular tachycardia. J Physiol 594, 567-593.

Yazawa, M., Hsueh, B., Jia, X., Pasca, A.M., Bernstein, J.A., Hallmayer, J., and Dolmetsch, R.E. (2011). Using induced pluripotent stem cells to investigate cardiac phenotypes in Timothy syndrome. Nature 471, 230-234.

Yu, J., Vodyanik, M.A., Smuga-Otto, K., Antosiewicz-Bourget, J., Frane, J.L., Tian, S., Nie, J., Jonsdottir, G.A., Ruotti, V., Stewart, R., et al. (2007). Induced Pluripotent Stem Cell Lines Derived from Human Somatic Cells. Science 318, 1917-1920.

Yuchi, Z., Lau, K., and Van Petegem, F. (2012). Disease mutations in the ryanodine receptor central region: crystal structures of a phosphorylation hot spot domain. Structure 20, 1201-1211. Yuchi, Z., Yuen, S.M., Lau, K., Underhill, A.Q., Cornea, R.L., Fessenden, J.D., and Van Petegem, F. (2015). Crystal structures of ryanodine receptor SPRY1 and tandem-repeat domains reveal a critical FKBP12 binding determinant. Nat Commun 6, 7947. 
Zaccolo, M. (2006). Phosphodiesterases and compartmentalized cAMP signalling in the heart. Eur J Cell Biol 85, 693-697.

Zaccolo, M., and Pozzan, T. (2002). Discrete Microdomains with High Concentration of cAMP in Stimulated Rat Neonatal Cardiac Myocytes. Science 295, 1711-1715.

Zhang, X.H., Haviland, S., Wei, H., Saric, T., Fatima, A., Hescheler, J., Cleemann, L., and Morad, M. (2013a). $\mathrm{Ca}(2+)$ signaling in human induced pluripotent stem cell-derived cardiomyocytes (iPS-CM) from normal and catecholaminergic polymorphic ventricular tachycardia (CPVT)-afflicted subjects. Cell calcium 54, 57-70.

Zhang, Y., Cao, N., Huang, Y., Spencer, C.I., Fu, J.D., Yu, C., Liu, K., Nie, B., Xu, T., Li, K., et al. (2016). Expandable Cardiovascular Progenitor Cells Reprogrammed from Fibroblasts. Cell stem cell 18, 368-381.

Zhang, Y., Matthews, G.D., Lei, M., and Huang, C.L. (2013b). Abnormal Ca(2+) homeostasis, atrial arrhythmogenesis, and sinus node dysfunction in murine hearts modeling RyR2 modification. Frontiers in physiology 4, 150.

Zhao, Y.T., Valdivia, C.R., Gurrola, G.B., Powers, P.P., Willis, B.C., Moss, R.L., Jalife, J., and Valdivia, H.H. (2015). Arrhythmogenesis in a catecholaminergic polymorphic ventricular tachycardia mutation that depresses ryanodine receptor function. Proceedings of the National Academy of Sciences of the United States of America.

Zhong, X., Sun, B., Vallmitjana, A., Mi, T., Guo, W., Ni, M., Wang, R., Guo, A., Duff, H.J., Gillis, A.M., et al. (2016). Suppression of Ryanodine Receptor Function Prolongs Ca2+ Release Refractoriness and Promotes Cardiac Alternans in Intact Hearts. Biochem J.

Zhou, H., Wu, S., Joo, J.Y., Zhu, S., Han, D.W., Lin, T., Trauger, S., Bien, G., Yao, S., Zhu, Y., et al. (2009). Generation of induced pluripotent stem cells using recombinant proteins. Cell stem cell 4, 381-384.

Zhou, T., Benda, C., Dunzinger, S., Huang, Y., Ho, J.C., Yang, J., Wang, Y., Zhang, Y., Zhuang, Q., Li, Y., et al. (2012). Generation of human induced pluripotent stem cells from urine samples. Nature protocols 7, 2080-2089.

Zhou, W., and Freed, C.R. (2009). Adenoviral gene delivery can reprogram human fibroblasts to induced pluripotent stem cells. Stem cells 27, 2667-2674.

Zoccarato, A., Surdo, N.C., Aronsen, J.M., Fields, L.A., Mancuso, L., Dodoni, G., Stangherlin, A., Livie, C., Jiang, H., Sin, Y.Y., et al. (2015). Cardiac Hypertrophy Is Inhibited by a Local Pool of cAMP Regulated by Phosphodiesterase 2. Circulation research 117, 707-719. 


\section{Acknowledgement}

I would like to take the opportunity to express my deepest gratitude to all people who supported me during my doctoral studies over the last years.

Special thanks go to Prof. Dr. Kaomei Guan for giving me the opportunity to be a doctoral student under her supervision and to investigate this interesting project, and for enormous support these years! I would like to thank my thesis committee members Prof. Dr. rer. nat. Kaomei Guan, Prof. Dr. med. Swen Hülsmann and Prof. Dr. rer. nat. Viacheslav Nikolaev for their great scientific support and helpful discussions in annual committee meetings.

I extend my sincere thanks to the other members of my examination board: Prof. Dr. mult. Thomas Meyer, Prof. Dr. Ralf Dressel, Prof. Dr. Dörthe Katschinski for agreeing to serve as my examiners.

I would like to extend my appreciation to the Ph.D. program of Molecular Medicine for giving me the opportunity to get an insight into various methods and skills courses.

I would like to thank my nice colleague Sarah Henze for working together happily in this intriguing CPVT project.

I am grateful to be working at the nice and friendly working places at the Department of Cardiology and Pneumology in Göttingen, and Stem Cell Unit in Universitätsmedizin Göttingen as well as Institute of Pharmacology and Toxicology in Technische Universität Dresden.

I am also very grateful to Dr. Tomasz Kolanowski and Dr. Christopher Antos for revising this thesis and Dr. Julia Sprenger for the support of the FRET technique and Yvonne Wiegräfe for the help of histological experiments.

I want to thank my family for their support during these years. I thank my wife Xiaojing for her patience and everlasting loving... 


\section{Curriculum Vitae}

Personal Information:

Name: Wener Li

Gender: Male

Nationality: Han

Date of Birth: 11.07.1986

Birth Place: Dawu, Hubei Province, P. R. China

Education:

2006-2010 Wuhan Bioengineering Institute

Bachelor Degree of Engineering

2010- 2013 South-Central University for Nationalities

Master Degree of Science

Since July 2013 Georg-August-Universität Göttingen

Promotion for Ph.D. 